\title{
Role of Serine hydroxymethyltransferase in cell cycle control and early Drosophila development
}

\author{
Doctoral thesis \\ Dissertation for the award of the degree \\ Dr. rer. nat. \\ in the GGNB program "Genes and Development" \\ at the Georg August University Göttingen \\ Faculty of Biology
}

submitted by

Franziska Winkler

born in Dresden, Germany

August 2015

revised version submitted in October 2016 


\section{Members of the Examination board}

\section{Members of the Thesis committee:}

Prof. Dr. Jörg Großhans (Supervisor, Reviewer)

Institute for Developmental Biochemistry, Medial School, University of Göttingen

Dr. Ronald Kühnlein (Reviewer)

Department of Molecular Developmental Biology, Max Planck Institute for Biophysical Chemistry

Dr. Arno Müller

Department of Cell and Developmental Biology, University of Dundee

Further members of the Examination board:

Prof. Dr. Ernst Wimmer

Department of Developmental Biology, University of Göttingen

Prof. Dr. Holger Bastians

Department of Molecular Oncology, University of Göttingen

Prof. Dr. Matthias Dobbelstein

Department of Molecular Oncology, University of Göttingen

Date of oral examination: 09.10 .2015 


\section{Affidavit}

I hereby declare that I prepared the PhD thesis "Role of Serine hydroxymethyltransferase in cell cycle control and early Drosophila development" on my own with no other sources and aids than quoted.

Nanostring experiments and the preparation for RNA sequencing were carried out by Dr. Hung-wei Sung.

The project "Analysis of centrosomal fluctuations reveal a role of Kinesin-1 in anchoring to the F-actin cortex" was carried out as a collaborative work. I performed imaging of centrosomes and nuclei including imaging of all WT and mutant embryos and injection procedures, except the imaging of azide injected and fixed embryos, which was carried out by Dr. Maheshwar Gummalla (Institute for Developmental Biochemistry, Medical School, Georg-August-University Göttingen). Image processing and computational analysis was done by Dr. Timo Aspelmeier (Institute of Mathmatical Stochastics, Georg-August-University Göttingen) and Lutz Künneke (Institute for Theoretical Physics, Georg-August-University Göttingen).

Franziska Winkler

Göttingen, 08.08.2015 



\section{Contents}

List of Figures $\quad$ xi

List of Tables $\quad$ xiv

Abbreviations $\quad$ XV

I Role of Serine hydroxymethyltransferase in cell cycle control and early Drosophila development 1

Summary 3

1 Introduction $\quad \mathbf{5}$

1.1 Switch in the mode of the cell cycle during the mid-blastula transition . . 5

1.2 DNA checkpoints and S phase control . . . . . . . . . . . . . . 10

1.3 Serine hydroxymethyltransferase . . . . . . . . . . . . . . . . . . . . . . 12

1.4 Aim of the work . . . . . . . . . . . . . . . . 17

2 Results $\quad 19$

2.1 The X238 mutation is located in the gene encoding for the enzyme Serine hydroxymethyltransferase . . . . . . . . . . . . . . . . . . 19

2.1.1 X238 creates a premature stop codon in the shmt gene . . . . . . 19

2.1.2 X238 mutant embryos can be rescued by introduction of a functional shmt gene . . . . . . . . . . . . . . . . . . . 19

2.2 Phenotypic description of the shmt mutants X238 and M281R . . . . . 21

2.2.1 The X238 mutant is not able to cellularise, although cellularisation markers are localised . . . . . . . . . . . . . . . . 21

2.2.2 SHMT reduction in clones does not affect cell proliferation . . . . 27

2.2.3 Nuclear morphology becomes disturbed in X238 mutants . . . . . 29

2.2.4 Loss of SHMT results in a premature stop of the syncytial cell cycle progression . . . . . . . . . . . . . 31

2.2.5 Characterisation of a second allele of shmt: M281R . . . . . . 33

2.2.6 The penetrance of the X238 phenotype depends on the age of the mother . . . . . . . . . . . . . . 35

2.2.7 The X238 phenotype is not dependent on the temperature . . . . 35 
2.3 Enzymatic function of SHMT is necessary for progression into cycle 14 and cellularisation . . . . . . . . . . . . . . . 38

2.4 Impaired DNA replication leads to a cell cycle phenotype in X238 . . . 42

2.4.1 Twine is not degraded in X238 . . . . . . . . . . . . . . . . 42

2.4.2 X238 phenotype depends on zygotic gene expression . . . . . . . . 44

2.4.3 Checkpoint activation in SHMT mutants . . . . . . . . . . . 45

SHMT mutants exhibit a nuclear retention of specific mRNAs . . 45

The X238 phenotype partially depends on the checkpoint kinase

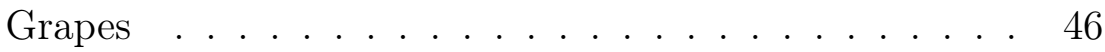

2.4.4 S phase progression in shmt mutants . . . . . . . . . . . 47

X238 embryos exhibit a prolonged S phase in cycle $13 \ldots$. . . . . 47

Temporal resolution of replication by PCNA-GFP . . . . . . . . 53

X238 mutants develop replication stress . . . . . . . . . . 55

Centrosome duplication is not affected in X238 mutants . . . . . 56

2.5 Nuclear localisation of SHMT . . . . . . . . . . . . . . 57

2.6 Injection of GFP-HP1 rescues the cellularisation and cell cycle phenotypes

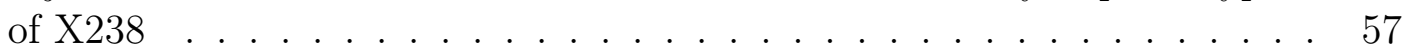

2.7 Gene expression in X238 embryos . . . . . . . . . . . . . 63

2.7.1 Zygotic genome is activated in X238 . . . . . . . . . 63

2.7.2 Maternal RNA degradation is similar between WT and X238 . . . 67

2.7.3 X238 embryos show a specific change in their gene expression profile for metabolic enzymes . . . . . . . . . . . . . . 67

\section{Discussion}

3.1 The enzymatic function of SHMT is required for early Drosophila development . . . . . . . . . . . . . . . . . . 73

3.2 SHMT depletion may activate the replication checkpoint by development of replication stress . . . . . . . . . . . . . . . . . . . 75

3.3 Nuclear SHMT may function in local thymidylate supply at the replication fork . . . . . . . . . . . . . . . . . 76

4 Materials and Methods $\quad 83$

4.1 Materials . . . . . . . . . . . . . . . . . 83

4.1.1 Reagents ........................ 83

4.1 .2 Commercial kits . . . . . . . . . . . . . . . . . . . . . . 83

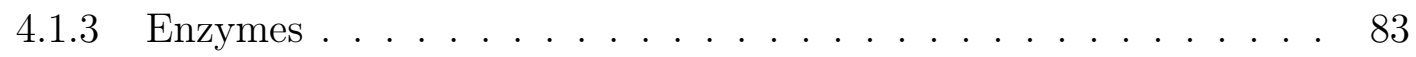

4.1 .4 Microscopy . . . . . . . . . . . . . . . . . . . 84

4.1.5 Chromatography ..................... 85

4.1 .6 Media . . . . . . . . . . . . . . . . . . 85

4.1.7 Buffers and solutions . . . . . . . . . . . . . . . . 85

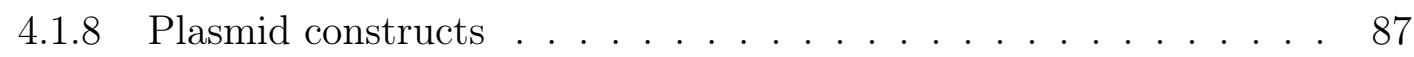

4.1 .9 Oligonucleotides . . . . . . . . . . . . . . . . . . . . 88 
4.1.10 Antibodies . . . . . . . . . . . . . . . . . . . . . 91

4.1 .11 Bacterial strains . . . . . . . . . . . . . . . . . 92

4.1 .12 Fly stocks . . . . . . . . . . . . . . . . . . . . . . . . . . . . . . . . . . . . . . 92

4.1 .13 Other materials . . . . . . . . . . . . . . . . . 97

4.1.14 Other equipment . . . . . . . . . . . . . . . 98

4.1 .15 Software . . . . . . . . . . . . . . . . . . 998

4.2 Methods . . . . . . . . . . . . . . . . . . . . . . . 99

4.2.1 DNA and RNA methods . . . . . . . . . . . . . . . . . 99

Polymerase chain reaction . . . . . . . . . . . . . . . . . . 99

Site-directed mutagenesis . . . . . . . . . . . . . . . 100

In-fusion cloning . . . . . . . . . . . . . . . . 100

Preparation of genomic DNA from bacterial cells . . . . . . . 100

Sequencing of plasmid constructs and PCR products . . . . . . 100

Cloning of GST-SHMT-His . . . . . . . . . . . . . . . 100

Cloning of pattB-SHMT-rescue . . . . . . . . . . . . . . 101

Cloning of alternate rescue constructs . . . . . . . . . . . . . . 101

Isolation and purification of DNA and RNA . . . . . . . . . 102

Quantitative Nanostring . . . . . . . . . . . . . . 103

RNA sequencing . . . . . . . . . . . . . . 103

4.2 .2 Protein methods . . . . . . . . . . . . . . . . . . . . 103

Western blot. . . . . . . . . . . . . . . . . 103

Expression and purification of proteins . . . . . . . . . . 103

4.2.3 Methods related to flies . . . . . . . . . . . . . . . . . . . 105

Fly genetics . . . . . . . . . . . . . . . . 105

Generation of germline clones . . . . . . . . . . . . 105

Generation of transgenic flies . . . . . . . . . . . 106

Fixation and immunostaining of Drosophila embryos . . . . . . . 106

EdU labelling of embryos . . . . . . . . . . . . . 106

Fluorescence in situ hybridisation . . . . . . . . . . . . . . . 106

Microinjection . . . . . . . . . . . . . . . . 106

Induction of clones in imaginal discs and follicle epithelium . . . . 107

Preparation and staining of imaginal discs and ovaries . . . . . . . 107

4.2.4 Microscopy and related methods . . . . . . . . . . . . . . . . . 108

Live imaging . . . . . . . . . . . . . . . . . . . 108

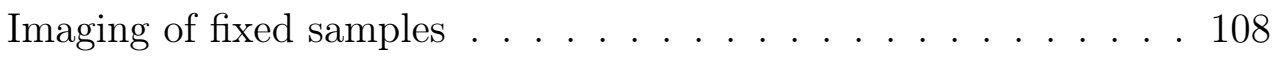

Fluorescence recovery after photo-bleaching . . . . . . . . . . . 108

Image processing and data analysis . . . . . . . . . . . . . . . 109

Appendix

Counts obtained in Nanostring experiments . . . . . . . . . . . 111

Lists of candidates identified in RNA sequencing . . . . . . . . . . 114 
Bibliography

II Fluctuation analysis of centrosomes reveals a cortical function of Kinesin-1

1 Introduction

1.1 The cytoskeleton in early Drosophila embryos . . . . . . . . . . . . . . . 134

1.2 Components of the cytoskeleton . . . . . . . . . . . . . 135

1.2 .1 Actin . . . . . . . . . . . . . . . . . . 135

1.2.2 Microtubules and centrosomes . . . . . . . . . . . . . . 137

1.2 .3 Microtubule motor proteins . . . . . . . . . . . . . . . 138

1.3 Aim of the work . . . . . . . . . . . . . . . . . 139

1.4 Contribution to the project . . . . . . . . . . . . . 139

2 Publication: Fluctuation analysis of centrosomes reveals a cortical function of Kinesin-1

3 Discussion

$\begin{array}{ll}\text { Bibliography } & 160\end{array}$

$\begin{array}{ll}\text { Acknowledgements } & 165\end{array}$

$\begin{array}{ll}\text { Curriculum vitae } & 167\end{array}$ 


\section{List of Figures}

1 Early development of Drosophila. . . . . . . . . . . . . . . . 5

2 Schematic representation of cellularisation. . . . . . . . . . . 7

3 Cell cycle models during early Drosophila development. . . . . . . . . . . 9

4 The ATR and ATM mediated checkpoints in early Drosophila development. 11

5 Cellular roles of SHMT in one-carbon metabolism. . . . . . . . . . . . . 13

6 The folate-mediated one-carbon metabolism is compartmentalised in the cytoplasm, mitochondria and nucleus. . . . . . . . . . . . . . . . . 15

7 Mapping of the X238 mutation. . . . . . . . . . . . . . 20

8 Scheme of the genomic region surrounding the shmt gene with the position of the X238 mutation indicated. . . . . . . . . . . . . . . 21

9 Depletion of SHMT disrupts invagination of the cellularisation furrow. . . 22

10 Localisation of the furrow canal marker Dia is not affected in the mutant. 23

11 Slam protein is localised at the invaginating furrow canal. . . . . . . . . . 24

12 Localisation of Dlg, a marker for the lateral membrane, is not altered in X238 embryos. . . . . . . . . . . . . . . . . . . . 25

13 Serendipity $\alpha(\operatorname{Sry}-\alpha)$, a late cellularisation protein, is not localised in X238 embryos. . . . . . . . . . . . . . . 26

14 Protein localisation of Eve reflects the expression pattern of WT embryos in early cellularisation. . . . . . . . . . . . . . . . 27

15 Induction of shmt-depleted clones in follicle epithelium does not interfere with proliferation. . . . . . . . . . . . . . . 28

16 Proliferation is not affected in shmt-depleted clones in imaginal discs. . . 29

17 Nuclei become misshaped in X238 mutants during cycle 13. . . . . . . . . 30

18 Kuk protein staining in X238 embryos reflects the defect nuclear morphology, although it is not present in all nuclei. . . . . . . . . . . . . 31

19 X238 embryos stop cell cycle progression in interphase 13. . . . . . . . . 32

20 Lengthening in cell cycle duration in X238 is similar to WT, but the cell cycle progression stops already in cycle 13. . . . . . . . . . . . . 33

21 The shmt mutant alleles X238 and M281R show a similar blastoderm phenotype. . . . . . . . . . . . . . . 34

22 The blastoderm phenotype depends on the age of the mother flies. . . . . 36

23 The X238 phenotype is not sensitive to the temperature. . . . . . . . . 36

24 Scheme of the genomic region surrounding the shmt gene with indicated transcripts, mutations and rescue constructs. . . . . . . . . . . . . . . 39 
25 Prediction of Drosophila SHMT structure aligned with rabbit cytosolic SHMT . . . . . . . . . . . . . . . . . .

26 Enzymatic function of SHMT is necessary for cell cycle progression and cellularisation. . . . . . . . . . . . . . . . . . 41

27 The cell cycle pause of X238 does not depend on Twine degradation. . . 43

28 X238 mutant embryos do not express Frs prematurely. . . . . . . . . . . 43

29 Blocking transcription with $\alpha$-Amanitin in X238 mutants leads to pro-

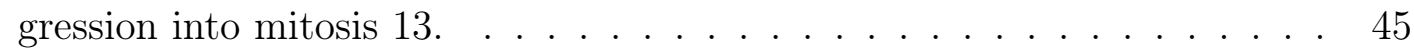

$30 k u k$ in situ hybridisation is specific for $k u k$ mRNA. . . . . . . . . . . . 46

$31 k u k$ mRNA localisation is altered in X238. . . . . . . . . . . . . 47

32 slam mRNA is retained in the nuclei in X238 embryos. . . . . . . . . . . 48

33 The X238 phenotype is partially dependent on the activation of the Grapes checkpoint. . . . . . . . . . . . . . . . . 49

34 EdU is incorporated ubiquitously in the syncytial blastoderm nuclei of WT embryos. . . . . . . . . . . . . . . . . . 50

35 Pattern of EdU incorporation in X238 is irregular and persists until latephase defect. . . . . . . . . . . . . . . . . 51

36 Magnified images of EdU staining. . . . . . . . . . . . . . . . 52

37 Centromere formation is not affected in X238 mutants. . . . . . . . . . 53

38 X238 embryos accumulate PCNA at the nuclear periphery. . . . . . . . 54

39 Replication stress occurs in cycle 13 in X238 embryos. . . . . . . . . . . . 56

40 Centrosome proliferation is not affected in the mutant. . . . . . . . . 56

41 SHMT signal is reduced in shmt mutants. . . . . . . . . . . . . . . 58

42 Antibody staining is specific for SHMT. . . . . . . . . . . 58

43 SHMT is localised predominantly in the cytosol. . . . . . . . . . . . . 59

44 Immunofluorescent stainings of S2 insect cells showing the localisation of SHMT and Lamin C before and after extraction. . . . . . . . . . . . . 60

$45 \mathrm{X} 238$ mutant embryos injected with GFP-HP1 progress into cycle 14 and undergo cellularisation. . . . . . . . . . . . . . . . 61

46 Effect of different concentrations of injected GFP-HP1 on the cellularisation phenotype of X238. . . . . . . . . . . . . . . 61

47 HP1 staining becomes irregular in X238 embryos. . . . . . . . . . . . . 62

48 RNA samples of the same genotype cluster together. . . . . . . . . . . 64

49 Internal Nanostring CodeSet controls for normalisation and background determination. . . . . . . . . . . . . . . . . . 64

50 Expression of the housekeeping genes Rpl21, RpLP2, and RpL32 is similar between WT an X238. . . . . . . . . . . . . . . . . . . 65

51 Expression of zygotic genes and zygotically controlled degradation of mRNA is activated in X238. . . . . . . . . . . . . . . 6 66 66

52 Maternal genes are degraded in X238. . . . . . . . . . . 67

53 MA plot for comparison of presyncytial WT and X238 expression profiles. 68 
54 MA plot for comparison of WT and X238 blastoderm stage expression profiles. . . . . . . . . . . . . . . . . . . . 68

55 Most enriched GO terms of differentially expressed genes of blastoderm staged embryos. . . . . . . . . . . . . . . . . . 69

56 Heat map with the 50 most differentially expressed genes in presyncytial staged embryos. . . . . . . . . . . . . . . . . . 70

57 Heat map with the 50 most significantly differentially expressed genes in blastoderm staged embryos. . . . . . . . . . . . . . . . . 71

58 Model for the role of nuclear SHMT in local thymidylate synthesis for DNA replication. . . . . . . . . . . . . . . . . . 81

59 Purification of GST-SHMT-His with a HisTrap HP column. . . . . . . . 104

60 Purification of His-GFP-HP1 with a HisTrap HP column. . . . . . . . . . 104

61 Purification of His-GFP-linker with a HisTrap HP column. . . . . . . . . 105

62 Schematic representation of centrosome cycle and its lateral and cortical interactions. . . . . . . . . . . . . . . . 136

63 Model for the interaction of centrosomes with the cortex. . . . . . . . 159 



\section{List of Tables}

1 X238 embryos stop their syncytial development in cycle 13. . . . . . . . 32

2 Increasing of cell cycle length is similar in WT and X238 . . . . . . . . . 32

3 The mutant alleles X238 and M281R display a similar cell cycle phenotype. 35

4 The cell cycle phenotype of M281R is similar to that of X238. . . . . . 35

5 The cell cycle phenotype of X238 mutant embryos is age-dependent. . . . 37

6 The ability of X238 mutant embryos to cellularise is dependent on the age of the mothers. . . . . . . . . . . . . . . . . . 37

$7 \quad$ Temperature does not influence the manifestation of the phenotype. . . . 37

8 In trans complementation of the lethal phenotype by the alleles M281R und E130Q. . . . . . . . . . . . . . . . . . . . . 40

9 Progression into cycle 14 requires enzymatic function. . . . . . . . . . . . 42

10 Cellularisation depends on enzymatical activity. . . . . . . . . . . . . . 42

11 X238 mutants blocked in transcription enter mitosis 13. . . . . . . . . . . 44

12 The X238 phenotype depends on zygotic transcription. . . . . . . . . . . 44

13 Mutation of the checkpoint kinase Grapes promotes entry into mitosis 13 in X238 embryos. . . . . . . . . . . . . . . . . . . . . . . . 49

14 A fraction of X238, Grapes double mutants showed a mitotic catastrophe phenotype similar to grapes mutants. . . . . . . . . . . . . . . . 49

15 Number of syncytial divisions is not changed upon overexpression of PCNA. 55

16 Overexpression of PCNA does not significantly change the length of syncytial cell cycles. . . . . . . . . . . . . . . . . . 55

17 Injection of GFP-HP1 rescues the cell cycle phenotype of X238. . . . . . 60

18 Cellularisation defect of shmt mutants can be rescued by injection of GFP-HP1. . . . . . . . . . . . . . . . . . 60

20 Buffers used in the study. . . . . . . . . . . . . . . . . . 85

21 Plasmids used in the study. . . . . . . . . . . . . . . 87

22 Oligonucleotides used in this study. . . . . . . . . . . . . . . . 88

23 Primary antibodies used for this thesis. . . . . . . . . . . . . 91

24 Stocks used for this thesis. . . . . . . . . . . . . . . . . . . . 92

25 Normalised counts of internal Nanostring CodeSet controls. . . . . . . . . 111

26 Normalised counts of the housekeeping genes Rpl21, RpLP2, and RpL32. 111

27 Normalised counts of zygotic genes. . . . . . . . . . . . . . . . . . . . 112

28 Normalised counts of transcripts of zygotically degraded mRNAs. . . . . 112

29 Normalised counts of maternally degraded mRNAs. . . . . . . . . . . . . 113 
30 Genes differentially expressed in presyncytial X238 mutants compared to WT embryos. . . . . . . . . . . . . . . . . . . . . . . 114

31 Genes differentially expressed in X238 mutants of cycle 13 compared to cellularising WT embryos. . . . . . . . . . . . . . . . . . . 115 


\title{
Abbreviations
}

\author{
${ }^{\circ} \mathrm{C} \quad$ degree Celsius \\ $\alpha$-aman $\quad \alpha$-amanitin
}

aa amino acids

Abl Abelson

ADMM alternating direction of multiplier method

Arp Actin-related proteins

ATM Ataxia-telangiectasia mutated

ATP adenosin triphosphat

ATR ATM and Rad3-related

appr. approximately

Bloom. Bloomington Stock Center

BSA bovine serum albumine

C1 one-carbon

Cdk Cyclin dependent kinase

CDS coding sequence

cell. cellularisation

Chk1 Checkpoint kinase 1

Chk2 Checkpoint kinase 2

CID Collision Induced Dissociation

col. colcemide

CV column volumes

$\Delta \quad$ delta-

D fluctuation parameter 


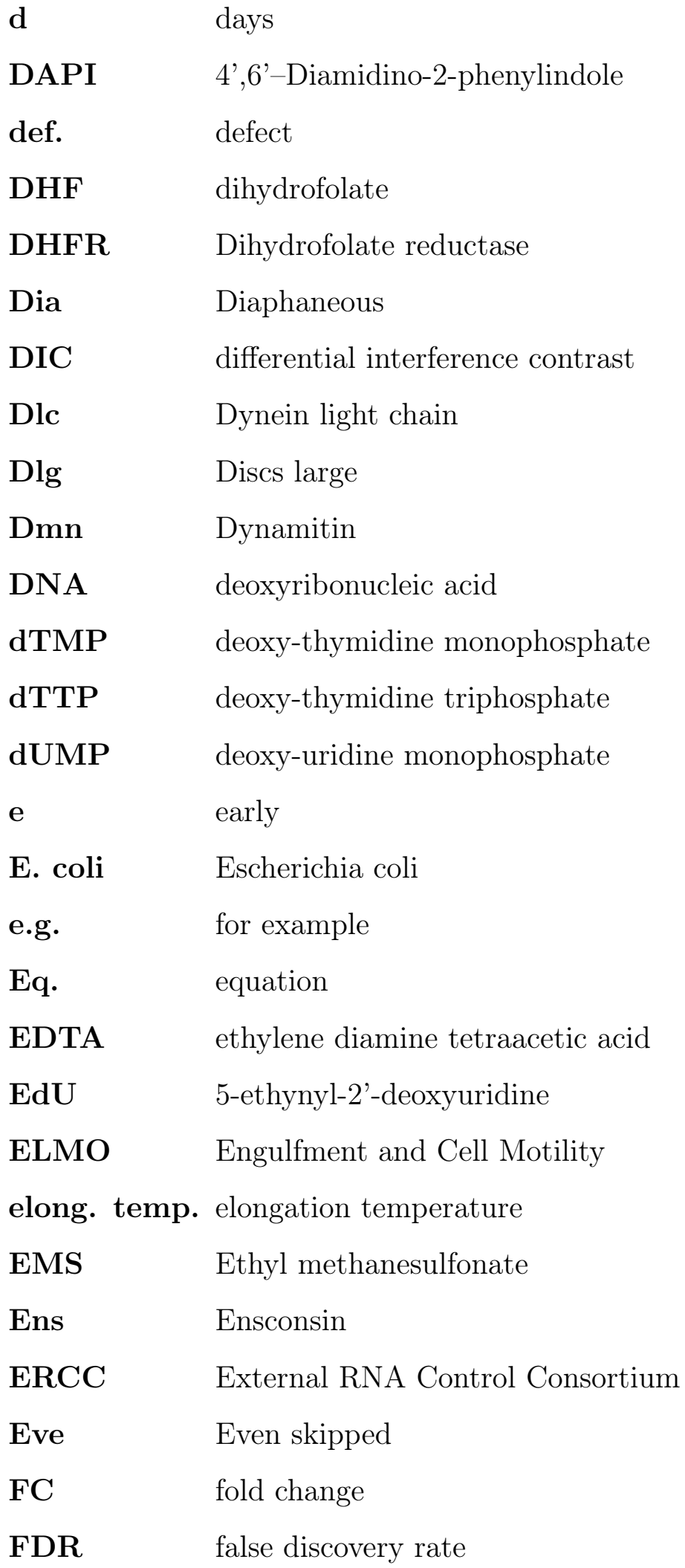


Fig. $\quad$ figure

Flp flippase

FRAP fluorescence recovery after photobleaching

Frs Frühstart

Frt flippase recognition target

GFP Green fluorescent protein

glc germ line clone

GLDC Glycine decarboxylase

Glu glutamic acid

GO gene ontology

GP guinea pig

grp Grapes

His Histone

HP1 Heterochromatin protein 1

hs heat shock promoter

hyb. sol. hybridisation solution

$\mathbf{H z} \quad$ Hertz

i interphase

i.e. id est

illum. illumination

inj. injected

IR ionising radiation

jasp. jasplakinolide

kDa kilo Dalton

Khc Kinesin heavy chain

Kin-1 Kinesin-1

Kuk Kugelkern

1 late 
lat. latrunculin

LC-MS/MS liquid chromatography-tandem mass spectrometry

$\mu \quad$ micro-

M Marker

m mitosis; meter

magn. magnification

MBT midblastula transition

min minute

Mnk Maternal nuclear kinase

mRNA messenger RNA

MSD mean square displacement

MTD maternal triple driver

mTHF methylenetetrahydrofolate

MTHFS Methenyltetrahydrofolate synthetase

mut.chr. mutant chromosome

$\mathbf{N} \quad$ number of

$\mathbf{N} / \mathbf{C} \quad$ nucleocytoplasmic

NA numerical aperture

neg. negative

nls nuclear localisation signal

ORF open reading frame

PAPS 3'-phosphoadenosine 5'-phosphosulfate

PAGE polyacrylamide gel electrophoresis

PBS phosphate buffered salt solution

PCNA Proliferating cell nuclear antigen

PCM pericentriolar matrix

PCR polymerase chain reaction

PIKK Phosphoinositide 3-kinase related kinases 
PLP pyridoxal-5-phosphate

pos. positive

PSF point spread function

R rabbit

rcSHMT rabbit cytosolic SHMT

RFP Red fluorescent protein

Rho Ras homologue gene family member

RNA ribonucleic acid

RNAi RNA interference

RpA Replication protein A

RpA70 Human replication protein A

RT room temperature

s second

S2 cells $\quad$ Schneider 2 cells

SAM S-adenosylmethionine

Sas4 Spindle assembly abnormal protein 4

Sas6 Spindle assembly abnormal protein 6

SDS sodium dodecylsulfate

sec second

SHMT Serine hydroxymethyltransferase

Slam Slow as molasses

Sry- $\alpha \quad$ Serendipity $\alpha$

STD standard deviation

SUMO Small ubiquitin-related modifier

Tab. table

TAL Transcriptome Analysis Laboratory

THF tetrahydrofolate

TYMS Thymidylate synthase 
uninj. uninjected

Utr Utrophin

UV ultraviolet

w white

WT Wild type 


\section{Part I}

Role of Serine hydroxymethyltransferase in cell cycle control and early Drosophila development 



\section{Summary}

The early development of Drosophila melanogaster occurs in a syncytium, where nuclei undergo mitotic divisions without accompanying cytokinesis. Early cleavage cycles are very short and consist only of $\mathrm{S}$ and $\mathrm{M}$ phases. In cycle 14, the cell cycle is paused and a G2 phase is introduced. During this extended interphase the embryo undergoes cellularisation, a modified form of cytokinesis which transforms the syncytial into a cellular blastoderm. Despite extensive investigations, regulation of cellularisation and progression of early cell cycles is not fully understood. The mutant X238 was identified in a screen for morphological defects in early development and contains a mutation in the gene CG3011 encoding the enzyme Serine hydroxymethyltransferase (SHMT). SHMT is a metabolic enzyme converting serine and tetrahydrofolate (THF) into glycine and methylene-THF (mTHF), which is the major one-carbon source for a large number of anabolic reactions including thymidylate and S-adenosylmethionine (SAM) synthesis. X238 embryos precociously arrest the cell cycle in interphase 13 and fail to undergo cellularisation, although zygotic gene expression is only weakly disturbed. Furthermore, nuclear morphology becomes disturbed in late cycle 13 of SHMT mutant embryos. Although early cellularisation markers are expressed and localised as revealed by immunostaining against Slow as molasses (Slam), Diaphanous (Dia), and Discs large (Dlg), cellularisation is not initiated. Moreover, its depletion causes the activation of both checkpoint kinases Chk1 (Grapes in Drosophila) and Chk2 (Maternal nuclear kinase - Mnk) in cycle 13, which finally stops the cell cycle progression. This checkpoint activation indicates the occurrence of replication stress, an interference of transcription and replication throughout an extended S phase 13. These results were confirmed by life imaging of RpA70-eGFP expressing Drosophila embryos, which accumulated RpA70-eGFP in foci within the nuclei during S phase 13 of X238 mutant embryos indicating replication stress. Inhibition of transcription by $\alpha$-amanitin as well as injection of the heterochromatin binding protein HP1 is able to rescue both the cell cycle and lethality phenotypes. This reduction of transcriptional activity, thereby reducing replication stress and postponing the activation of checkpoint kinases, may allow the mutant embryos to finish DNA replication in $\mathrm{S}$ phase 13. Consistent with the current literature, in which it was shown that SHMT functions at the localised thymidylate supply at the nuclear periphery of mammalian cells and that its mutations causes mis-incorporation of nucleotides, the results of this thesis lead to the conclusion that the blastoderm defect of X238 could be caused by an interference with DNA replication due to a lack of localised thymidylate synthesis. This could induce stalling of replication forks at the nuclear periphery, which activates the DNA replication checkpoint and stops cell cycle progression. 



\section{Introduction}

\subsection{Switch in the mode of the cell cycle during the mid-blastula transition}

\subsubsection{The early development of Drosophila melanogaster}

The early embryonic development of Drosophila melanogaster, as of the majority of insects, occurs in a syncytium. The nuclei divide without an accompanied cytokinesis. This results in a syncytial embryo with finally about 6000 nuclei in a common cytoplasm (Figure 1). The nuclei undergo thirteen rounds of mitotic divisions [55, 181]. During the first three divisions nuclei are clustered in the anterior third of the embryo [84]. In cycles 4 to 6 , the nuclei evenly distribute along the embryo length. This process is called axial expansion, and is dependent upon actin-myosin contractions [123, 165]. Cortical migration starts in cycle 8 , the first nuclei arrive at the posterior cortex in cycle 9 . These nuclei are germline precursors and form pole cells in cycle 10. The cortical movement occurs in a microtubule-dependent stepwise manner [165, 181]. It is assumed that the force for cortical migration is generated by plus-end-directed microtubule motors acting on neighbouring microtubule asters $[13,55]$. This hypothesis is supported by the observation that centrosomes, which are not associated to nuclei, also migrate to the cortex. The cortical nuclei form a monolayer, the syncytial blastoderm, and perform four more rounds of division. Whereas early cell cycles are very rapid with eight minutes on an average, interphase length becomes gradually increased during nuclear cycles 10 to 13 [181]. Cycle 14 consists of an extended interphase, which lasts about one hour. During this period, cellularisation proceeds. Cellularisation is a modified form of cytokinesis,

A

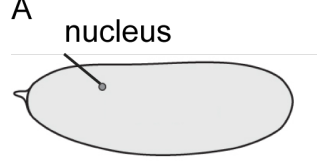

B

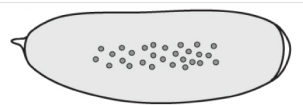

C

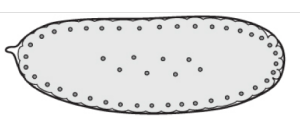

D

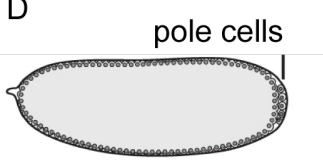

E

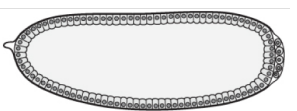

Figure 1: Early development of Drosophila. Fusion of egg and sperm nuclei induce rapid nuclear divisions, which occur in a common cytoplasm (A). The first seven nuclear cycles take place in the interior of the egg (B), cortical migration starts in cycle $8(\mathrm{C})$. At the cortex, syncytial blastoderm and pole cells are formed (D). During cellularisation in cycle 14, plasma membranes invaginate between the nuclei and form the cellular blastoderm embryo (E). Modified from: L. Wolpert - Principles of Development, 5. ed.[172]. 
which transforms the syncytial into a cellular blastoderm [100]. Immediately after cellularisation has finished, gastrulation is initiated. Within this process of coordinated cell rearrangements the monolayer of cells is transformed into layers giving rise to endoderm, mesoderm and ectoderm (reviewed in Leptin, 1999 [89]).

In synctial blastoderm stage, actin is redistributed throughout cell cycle progression [79]. Actin is accumulated at the embryonic cortex. In interphases it forms actin caps above each nucleus. During mitosis, in prophase and metaphase, plasma membrane invaginates between the nuclei to a depth of approximately (appr.) $8 \mu \mathrm{m}$. Actin is redistributed from the cap structure to the leading edge of the invaginating furrow. At the end of mitosis, at telophase, the plasma membrane regresses and actin arranges again into actin caps. These plasma membrane is called metaphase furrow or pseudocleavage furrow and prevents spindles from adjacent nuclei to fuse with each other [150]. At the onset of cellularisation, similar to previous interphases, a pair of centrosomes is located apically to each nucleus (Figure 2). Microtubules emanate from the centrosomes and form an inverted basket-like structure around the nuclei. Actin redistributes similarly to previous cycles from the cap structure above the centrosomes into the tip of the invaginating furrow, the furrow canal [61].

The cellularisation process is subdivided into four phases, which can be distinguished from each other by morphological features and the different speed of furrow invagination $[81,88]$. In phase 1 the nuclei elongate and the cellularisation furrow starts to invaginate [61]. Microvilli are accumulated at the cortical region above the nuclei, whereas they are absent at the furrow canal, the region at the middle of adjacent nuclei [88]. Phase 2, in which the nuclei complete elongation, is characterised by a slow membrane invagination. During phase 3 the invaginating furrow reaches the basal plane of the nuclei. Furrow invagination is completed in phase 4 , at a depth of about $35 \mu \mathrm{m}$. The furrows start to contract laterally and to enclose the nuclei into cells. This process is called basal closure.

During invagination the membrane is compartmentalised into an apical, lateral and basal domain separated by apical and basal adherens junctions, respectively. Mavrakis and colleagues revealed that the plasma membrane is already polarised in syncytial blastoderm stage [99]. They could identify two distinct domains above and at the lateral region of the nuclei. Invagination of the plasma membrane and domain formation are mainly controlled by two processes: organisation of the cytoskeleton and membrane trafficking [2]. Factors such as Rho1, RhoGEF2, Diaphaneous (Dia) and Abelson (Abl) control the organisation of the cytoskeleton [3, 38, 68, 70, 114], whereas membrane trafficking involves the insertion of polarised plasma membrane and regulated endo- and exocytosis (reviewed in Albertson et al., 2005 [5]). Many key regulators of membrane trafficking exhibit essential roles during cellularisation. Some of the important factors in cellularisation are discussed below. 
A

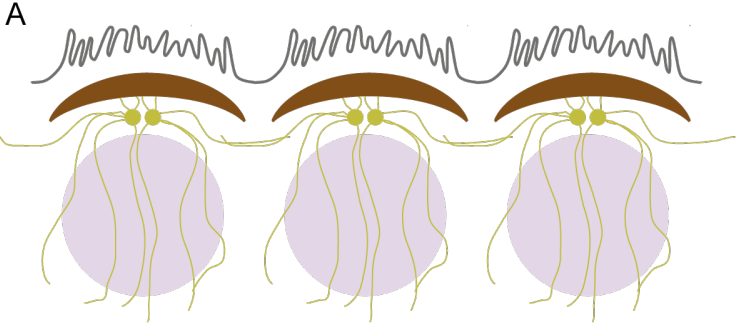

C
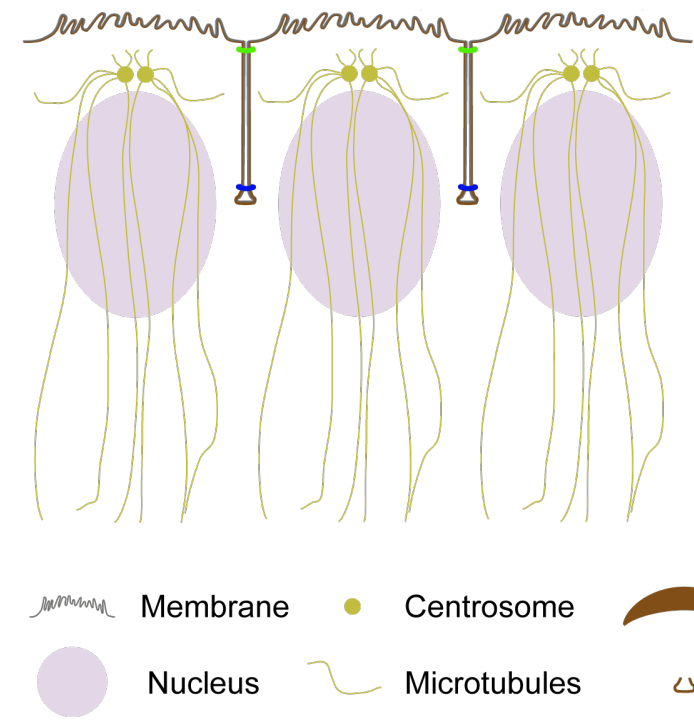

- Centrosome

Nucleus

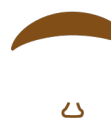

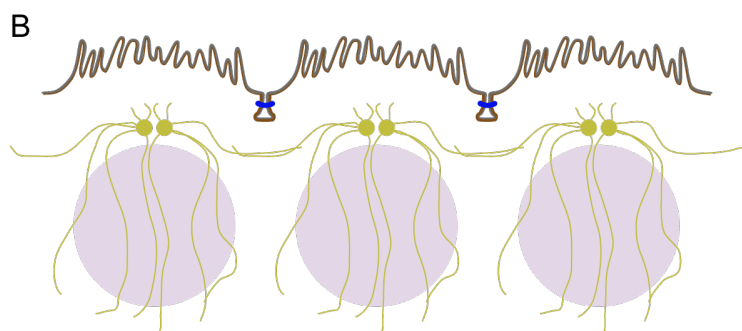

D

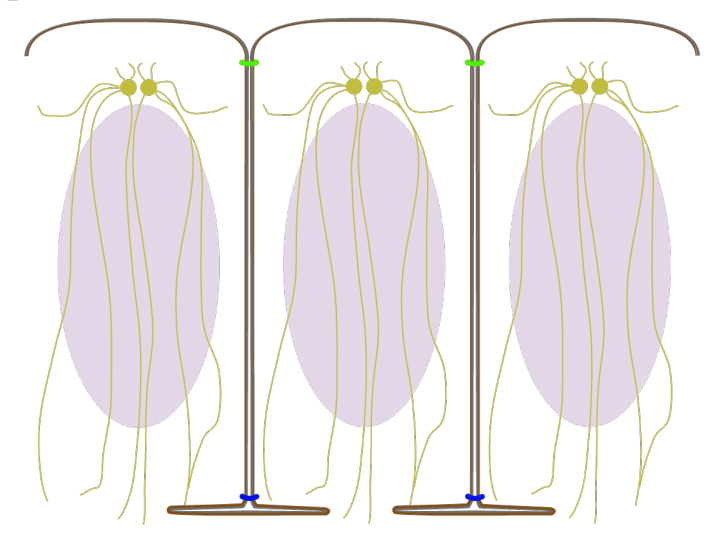

Actin cap

- Apical adherens junction

Actin at furrow canal

- Basal adherens junction

Figure 2: Schematic representation of cellularisation. At the beginning of cycle 14 interphase, actin forms caps apical to nuclei and centrosomes (A). Furrow canal and basal adherens junctions are built at onset of cellularisation (B). Furrow invaginates between the nuclei and apical adherens junctions are formed (C). Cellularisation front reaches a depth of appr. $35 \mu \mathrm{m}$ and encloses the nuclei in cells in a process called basal closure (D).

Formins are a protein family, which associates to the plus end of actin filaments and mediate actin nucleation and polymerisation (reviewed in Chesarone et al., 2010 [35]). One of these formin proteins is Dia. Beside its role in actin nucleation and polymerisation, Dia is also known to regulate microtubule stability $[77,115,167]$. During syncytial blastoderm stage and cellularisation Dia is localised to the tip of the invaginating furrow. Embryos carrying a hypomorphic dia mutation show severe morphological defects after cycle 11. Actin protein is missing from pseudocleavage furrows. Recruitment of other furrow components such as Anilin, Peanut, and Myosin II is also affected [3]. Dia mutant embryos show defects in morphology and positioning of nuclei, resulting in misshaped nuclei, cells without or with multiple nuclei [3]. Furthermore, baso-lateral polarity establishment is affected [178].

Slow as molasses (Slam) is a zygotically expressed gene, which is important for the initiation of membrane invagination. It is weakly expressed during syncytial blastoderm 
stage. At the onset of cellularisation its expression is strongly increased. Zygotically slam deficient embryos exhibit a defect in the slow phase of cellularisation, which results in delayed furrow invagination [87]. Embryos lacking both maternal and zygotic slam do not initiate furrow invagination $[143,2]$. Furthermore, it was shown that Slam has a second role in polarity establishment, since embryos lacking the zygotic contribution of slam do not form basal junctions [87].

Discs large (Dlg) is a membrane-associated protein harbouring multiple PDZ domains, which are important protein-binding domains. It was identified as a tumor suppressor gene regulating cell shape and proliferation in imaginal epithelia of Drosophila [174]. It belongs to the conserved protein family of membrane associated guanylate kinases, although it lacks critical amino acids for a functional kinase activity $[174,175]$. It has been shown that Dlg is essential to prohibit cell growth and to maintain cell adhesion and cell polarity both in embryonic and adult tissues [63, 117, 173, 174, 176].

Sry- $\alpha$ is a zygotic gene expressed from syncytial blastoderm stage until gastrulation stage [134]. Sry- $\alpha$ plays an important role in cellularisation and colocalises with F-actin. Mutant embryos show cytoskeletal defects resulting in multinuclear cells assuming a role in the localisation of membrane furrows during syncytial blastoderm and cellularisation development [133].

\subsubsection{Mid-blastula transition: the switch from maternal to zygotic control of development}

Early embryonic development of most animals, which lay externally developing eggs, is characterised by a cleavage stage, in which the embryo is subdivided into an increasing number of smaller cells by synchronous and rapid cell divisions. In Drosophila, the cleavage cycles are extremely short due to the lack of gap phases (Figure 3). Maternal load of transcripts and proteins allows to bypass zygotic transcription, which would normally occur in the gap phases. Cell cycle becomes gradually longer from cycle 8 by introducing a G2 phase, until reaching a length of appr. 20 min in cycle 13. In cycle 14, the cell cycle pauses for appr. one hour to allow cellularisation. Zygotic genome is gradually activated. Initial transcription can be detected already in presyncytial cycles and increases during syncytial cycles $[39,108,156]$. Full zygotic transcription is reached in cycle 14. After cellularisation, the embryo enters gastrulation and looses the synchrony of the cell cycle. This switch between maternal and zygotic control of development is called midblastula transition (MBT) $[106,107]$. The MBT has been characterised in various organisms (e.g. Drosophila, Xenopus, and Danio) [45, 78], revealing that the number of cleavage cycles before MBT is very precise and species-specific (Drosophila exhibits 13, Xenopus 12, and zebrafish 11 cycles) [78, 179]. 

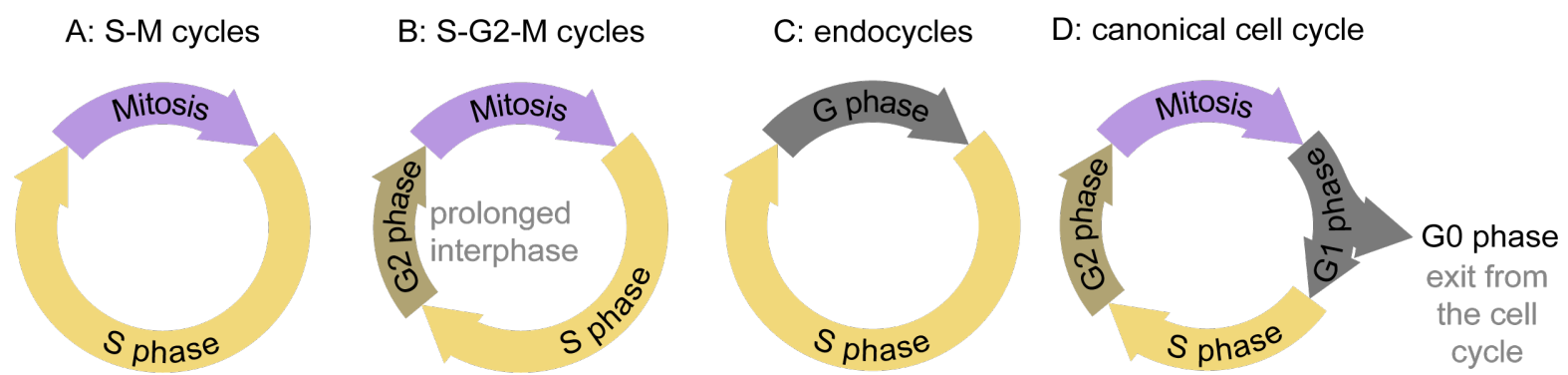

Figure 3: Cell cycle models during early Drosophila development. (A) Cleavage cycles 1 to 8 contain only S and M phases (S-M cycles). (B) During syncytial blastoderm, a G2 phase is introduced, leading to a progressive increase in cell cycle duration (S-G2-M cycles). (C) Endocycles consisting of $\mathrm{S}$ and $\mathrm{G}$ phases are required for some instances of organogenesis and are characteristic for yolk nuclei. (D) Canonical cell cycle containing of G1, S, G2 and M phase. Cells, which will not divide further, enter G0 phase. Modified from Budirahardja \& Gönczy, 2009 [33].

One factor controlling the timing of MBT is the nucleocytoplasmic $(\mathrm{N} / \mathrm{C})$ ratio. Analysis of Drosophila embryos with different ploidies showed that cell cycle pauses, when the ratio of DNA to cytoplasm has reached a specific threshold [45, 124]. Tetraploid embryos stop one cleavage cycle earlier, whereas haploid embryos undergo one more. This observation led to a titration model, published by Newport and Kirschner in 1984. This model proposed that chromosomes (or DNA in general) titrate specific rate-limiting cytoplasmic factors controlling DNA replication [105, 120]. In early cleavage cycles the $\mathrm{N} / \mathrm{C}$ ratio is low, which supports the progression of the cell cycle. Since no cell growth occurs, the $\mathrm{N} / \mathrm{C}$ ratio becomes higher with further cycles and eventually reaches a threshold, at which the cell cycle is stopped. Experiments manipulating the DNA content in embryos revealed that this threshold is reached with a DNA content of about $70 \%$ of the DNA amount, which is normally contained in cycle 14 [94]. However, the decision to stop cell cycle is not made by the embryo as a whole. At a DNA content near the threshold, the authors observed a patchy situation with embryos containing areas of different nuclear density. On the other hand, the nuclei are also not making this decision individually, otherwise these embryos would have a salt-and-pepper-like distribution of nuclei in earlier and later cell cycles. So nuclei communicate locally, which makes them collectively decide when to stop the cell cycle [93].

An important protein controlling the pausing of the cell cycle in G2 phase is the protein phosphatase Cdc25. It dephosphorylates Cyclin dependent kinase (Cdk) 1 and thereby activates the cyclin-Cdk1 complex, which promotes progression of the cell cycle into mitosis [46, 48, 126, 127, 147]. Drosophila has two Cdc25 homologues, String and Twine. Both proteins are maternally deposited and finally degraded in cycle 14 by zygotically controlled posttranslational mechanisms, but their degradation timing is different [42]. The degradation rate of String is gradually increased during the syncytial blastoderm cycles. Twine, however, is degraded specifically at the onset of cycle 14 . The degradation 
of both proteins is dependent on the $\mathrm{N} / \mathrm{C}$ ratio, but String degradation further depends on the activity of the DNA replication checkpoint. Edgar and colleagues demonstrated that overexpression of Twine can lead to an extra cleavage division, whereas its depletion can induce a premature stop of the cell cycle [44, 49].

\subsection{DNA checkpoints and S phase control}

\subsubsection{S phase progression}

Cleavage cycles are characterised by unusually fast $\mathrm{S}$ phases. In preblastoderm cycles of Drosophila, DNA replication of the whole genome is finished in $3.4 \mathrm{~min}$. The mainly dispersed chromatin contains numerous origins of replication, which initiate DNA replication synchronously [27]. S phase duration is prolonged during syncytial blastoderm by a gradual reduction of the number of replication origins and establishment of early and late replication [135]. The majority of this global changes in replication behaviour occur in S phase 14, which lasts 50 min, more than ten times longer than preblastoderm cycles. Coincidentally, the embryo degrades the majority of maternal transcripts and proteins and undergoes MBT. Although degradation of most maternal transcripts is independent of zygotic transcription and occurs also in unfertilised eggs, there is a specific group of maternally provided genes, which is degraded by zygotically transcribed gene products [28, 151]. String, twine, and smaug belong to this group. Zygotic gene expression and replication checkpoints mediated by Grapes and Drosophila maternal nuclear kinase (Mnk) are important to slow down cell cycle progression in the syncytial blastoderm and to pause it in cycle 14. The G2 phase in cycle 14 is introduced by the loss of maternal Cdc25 [47, 112]. Progress into mitosis 14 is temporally and spatially controlled by zygotic transcription of Cdc25 [47, 48, 54].

\subsubsection{S phase control: DNA damage and DNA replication checkpoints}

DNA checkpoints detect abnormal DNA structures or DNA damage, coordinate cell cycle progression with DNA repair mechanisms and induce apoptosis [1]. The activation of cell-cycle checkpoints involves three classes of proteins: sensor proteins, which recognise damaged DNA and relay a signal to transducer proteins, which amplify this signal by modulating downstream target effector proteins, which actually prevent the cell cycle progression [111].

PIKK (phosphoinositide 3-kinase related kinases) family proteins belong to the transducer protein group [157]. Two members of this protein family are Ataxia-telangiectasia mutated (ATM) and ATM and Rad3-related (ATR). Mei-41 was implicated to be Drosophila homologue of both ATM and ATR kinases [72], recent data indicate tefu as the 


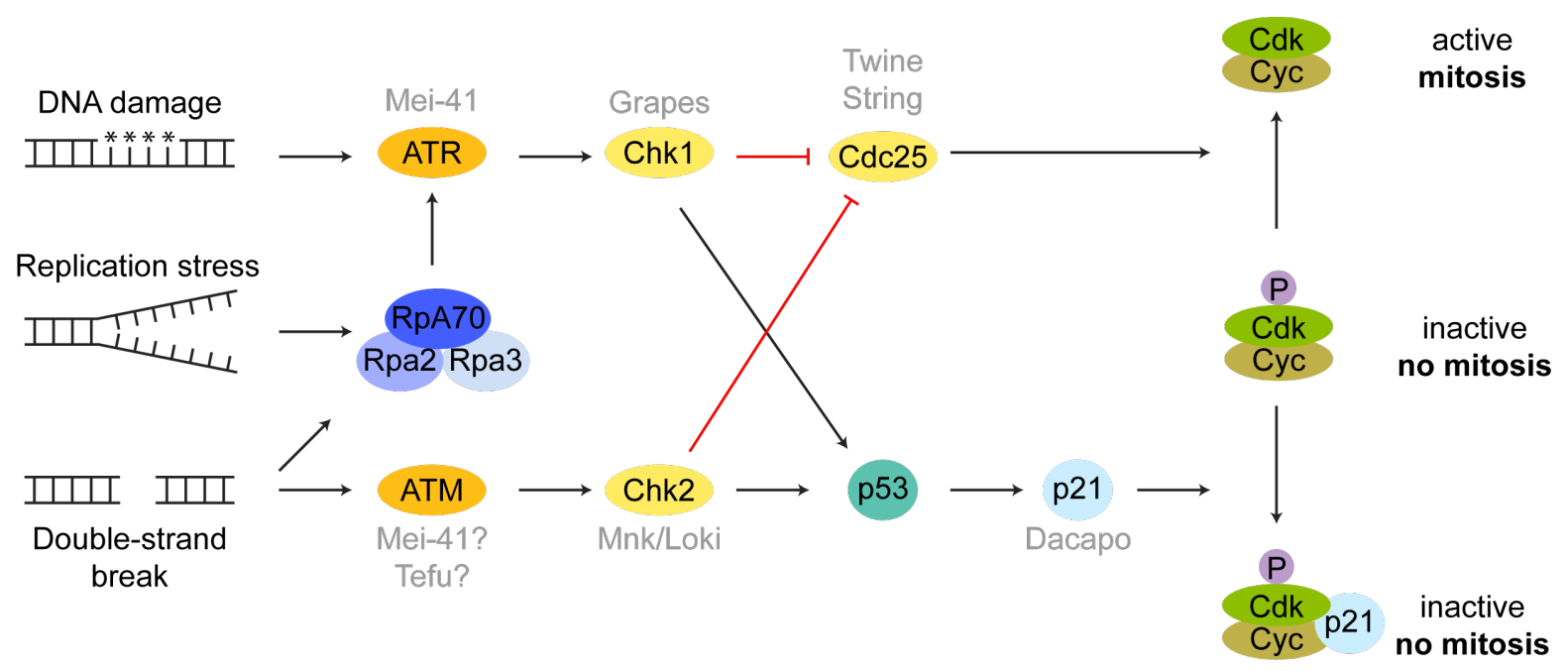

Figure 4: The ATR and ATM mediated checkpoints in early Drosophila development. The ATR (Mei-41 in Drosophila) and ATM kinases recognise DNA damage and replication stress either directly or via the accumulated Replication protein A (RpA) complex and signal it to the checkpoint kinases Chk1 (Grapes in Drosophila) and Chk2 (in Drosophila known as Mnk or Loki). Both kinases phosphorylate the Cdc25 phosphatase (Drosophila has two homologues: Twine and String), which is then degraded or prevented from nuclear import. According to this, the Cdk-cyclin (Cyc) complex is kept phosphorylated and inactive, which prevents the cell cycle progression into mitosis. Furthermore, the checkpoint kinases can activate the tumor suppressor protein $\mathrm{p} 53$, which induces the transcription of p21. P21 in turn also inhibits Cdk activity. If different from mammalian nomenclature, the Drosophila gene names are indicated in grey. Modified from DeVeylder et al., 2007 and Zou \& Elledge, 2003 [40, 185].

ATM homologue making Mei-41 to the homologue of ATR [24, 121, 138, 142]. Initial response to DNA damage or replicative stress is mainly carried out by ATR, which targets proteins such as H2AX, p53 and the checkpoint kinase 1 (Chk1) (Figure 4) [18]. Chk1 targets the Cdc25 phosphatase, which is an activator of Cdk proteins. Cdk proteins are necessary to promote entry into mitosis. Cdc25 phosphorylation by Chk1 triggers its degradation, which leads to a slowdown of DNA replication and prevents entry into mitosis [14]. Furthermore the checkpoint kinases can activate the tumor suppressor protein $\mathrm{p} 53$, which in turn activates the transcription of $\mathrm{p} 21$. This protein is a second inhibitor of Cdc25 phophatase. A second checkpoint kinase is Chk2. In fission yeast, it was shown that Chk1 is the main effector kinase in the DNA-damage-response pathway, whereas Chk2 is involved in the replication-checkpoint pathway [30, 103]. However, there are also reports that the protein functions partly overlap. Upon incomplete DNA replication, Chk1 becomes phosphorylated and arrests the cell cycle, if Chk2 is absent [29, 92, 103].

Under laboratory conditions, checkpoint genes are often non-essential for viability and fertility. However, some of these genes are crucial for the development of the organism 
$[32,155]$. grapes, the Drosophila homologue of the Chk1 gene, was identified as a maternally required gene for the MBT in order to coordinate the upregulated transcription activity with DNA replication [28, 56, 57, 137, 149]. grapes mutants fail to prolong the cell cycle during the syncytial blastoderm stage. Furthermore grapes mutant embryos do not stop the cell cycle progression in cycle 14. This results in a mitotic catastrophy in that incompletely replicated chromosomes are torn apart in mitosis 14. Mutants of mei-41, the Drosophila homologue of the ATR kinase, show a similar phenotype [72, $136]$.

The Drosophila protein Mnk is homologues to Chk2 and was shown to be necessary for the ionising radiation (IR) induced phosphorylation of $\mathrm{p} 53$, which subsequentially activates p21 (Dacapo in Drosophila) transcription and thereby inhibition of the Cdkcyclin complex (Figure 4) [31, 113, 118]. Otherwise, mnk embryos develop normally with no morphological defects in blastoderm embryos. Mutation of mnk in the grapes background suppresses both the mitotic catastrophy and cellularisation phenotypes of grapes, but does not restore cell cycle length or a functional replication checkpoint [154]. This shows that the morphological defects that are visible in grapes mutants depend on mnk. Furthermore, the double mutants exit mitosis 13 without chromosome separation, so interphase 14 has the same nuclear density as interphase 13 [42]. Cellularisation is initiated after several attempts of the embryo to undergo mitosis. These attempts are coupled to additional rounds of replication [98].

\subsection{Serine hydroxymethyltransferase}

\subsubsection{Enzymatic function of SHMT}

Serine hydroxymethyltransferase (SHMT, EC 2.1.2.1) converts serine into glycine and tetrahydrofolate (THF) into methylenetetrahydrofolate (mTHF), which is the major source of one-carbon units for numerous anabolic pathways in the cell $[9,58,102]$ (Figure 5). Beside their function in protein translation, the amino acids serine, glycine, and methionine have specific functions in cellular processes. Serine is necessary for the synthesis of phosphatidylserine, so the $\mathrm{C} 1$ pathway can influence cell membrane synthesis and maintenance. Glycine is involved the synthesis of purines, so it is indirectly involved in DNA and RNA biosynthesis. Methionine can be adenylated to S-adenosylmethionine (SAM), which is an important cofactor for methylation reactions [74]. SHMT can form a complex with the enzyme Dihydrofolate reductase (DHFR) and Thymidylate synthase (TYMS). In this complex, SHMT provides 5,10-mTHF for the synthesis of thymidylate, which is a precursor for dTTP biosynthesis [74].

A second reaction, catalysed by the cytosolic isoform of SHMT, is the conversion of mTHF into 5-formyl-THF. This irreversible reaction was named futile cycle [146]. 5- 


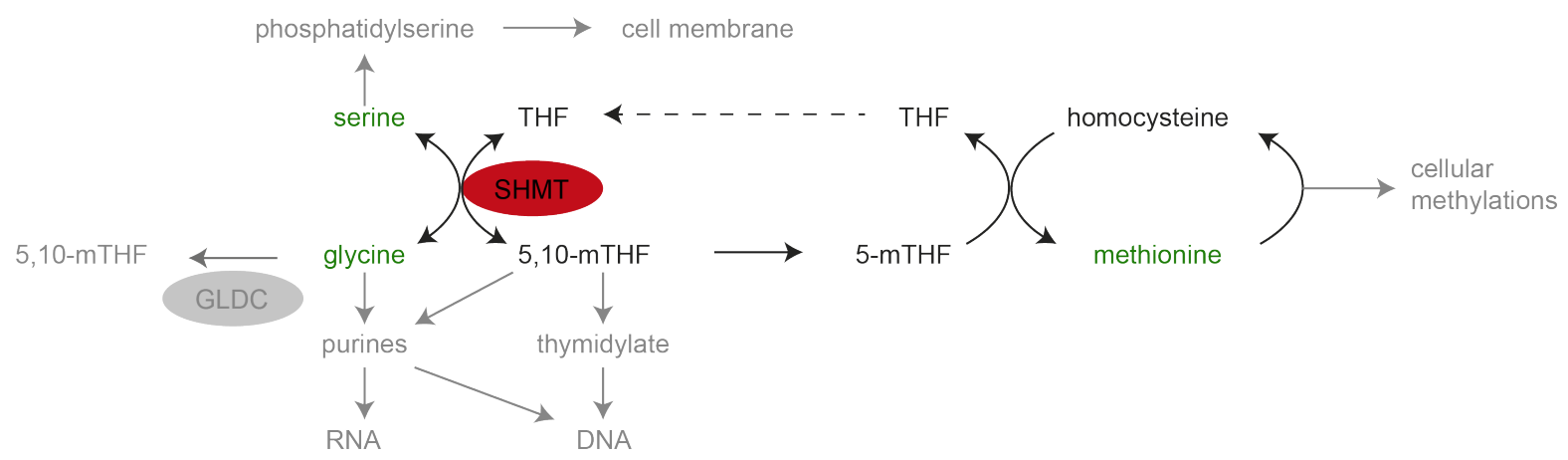

Figure 5: Cellular roles of SHMT in one-carbon metabolism. Serine hydroxymethyltransferase (SHMT) interconverts the amino acids serine and glycine. Concurrently, tetrahydrofolate (THF) is converted into 5,10-methylenetetrahydrofolate (5,10-mTHF). THF is regenerated through the modification of 5,10-mTHF into 5-methylenetetrahydrofolate (5-mTHF), which is then transformed into THF. Thereby homocysteine reacts to methionine. The reaction catalysed by SHMT influences several cellular processes. The amino acids serine, glycine, and methionine are necessary for protein synthesis (depicted in green). Other pathways, which involve components of the SHMT-mediated C1 metabolism are shown in grey. Serine can be used for the synthesis of phosphatidylserine, thereby the C1 metabolism could influence cell membrane synthesis and maintenance. 5,10-mTHF is needed for the de novo thymidylate pathway, and participates as well as glycine in the purine synthesis pathway. So, C1 metabolism has an indirect effect on DNA and RNA biosynthesis. Side products, which are built during the methionine cycle, are necessary for cellular methylation reactions. Furthermore, glycine can be converted to 5,10-mTHF, which is catalysed by the enzyme Glycine decarboxylase GLDC). Modified from Snell et al., 2000 [141].

formyl-THF is involved in the maintenance of one-carbon homeostasis due to its inhibitory function on SHMT [66, 146]. It can also serve as a storage form of THF cofactors [52]. The enzyme Methenyltetrahydrofolate synthetase (MTHFS) can convert 5-formylTHF into 5,10-methenyl-THF and thus mobilise it again for THF-dependent reactions.

\subsubsection{Evolution of SHMT}

SHMT is a highly conserved protein, both in prokaryotic and eukaryotic organisms [4]. In prokaryotes the enzyme is encoded by the glyA gene. It is assumed that the cytosolic isoform of eukaryotic SHMT is derived from the glyA gene product. The mitochondrial isoform originates from the gene encoding the cytosolic SHMT [41]. Crystal structure analysis revealed that prokaryotes express SHMT enzymes, which form a dimeric structure [132], whereas the eukaryotic enzyme evolved a homotetrameric structure, which consists of a dimer of two tightly bound dimers [11]. 


\subsubsection{SHMT localisation in the cell}

Mammalian cells contain SHMT isoenzymes in the cytosol and in the mitochondria (Figure 6). The cytoplasmic enzyme is encoded by shmt1, while shmt2 provides the mitochondrial protein $[62,65,145]$. In the mitochondria the SHMT-catalysed reaction is primarily used for glycine biosynthesis, thereby generated one-carbon units are exported into the cytosol as formate, which supports the cytoplasmic one-carbon metabolism [12, 145]. Biosynthesis of thymidylate, purines, as well as remethylation of homocysteine to methionine take place in the cytosol [9]. Studies using isotope tracers indicated that SHMT1 is preferentially generating mTHF for the thymidylate pathway [74]. This pathway involves the enzymatic function of three proteins: SHMT1, DHFR and TYMS. The mTHF units, which are generated by SHMT, are utilised as one-carbon donor for the conversion of dUMP to thymidylate catalysed by DHFR. mTHF is thereby converted into dihydrofolate (DHF), which is regenerated to THF by DHFR [9].

Detailed subcellular analysis revealed that the enzymes of the thymidylate pathway are also localised in the nucleus (Figure 6). This nuclear translocation is mediated by SUMOylation and is cell cycle-specific in $\mathrm{S}$ and G2/M phases. Additionally it occurs as a response to UV-induced DNA damage [7, 9, 59, 171]. It is assumed that the enzymes of the thymidylate pathway form a tight complex at the nuclear lamina. Stover and coworkers could show that all three enzymes colocalise with Lamin B1, which is dependent on SHMT [10]. Furthermore, they identified an interaction of SHMT1 with PCNA as well as other proteins involved in DNA replication and repair and its enrichment at sites of replication initiation and elongation [10,171]. Stover et al. proposed that the de novo thymidylate synthesis pathway is located at replication forks to directly support processive incorporation of dTTP into DNA during replication [74, 171]. The reversible compartmentalisation of the thymidylate pathway from cytosol to nucleus is an example for common mechanism to regulate cellular processes by preferential partioning of substrates to specific enzymes and enzyme complex. Compartmentalisation can establish specific locations to separate a pathway from other enzymes and enzyme complexes, which would compete for these substrates. This competition is very evident in folate metabolism. Enzymes, which require folate cofactors, are highly concentrated in the cell and often exceed the concentrations of total folates [131, 148]. Therefore, enzymes involved in biosynthesis of purine, thymidylate and SAM compete for this limited pool of folate-activated one-carbon units. The compartmentalisation of the thymidylate pathway to replication forks at the nuclear envelope during S-phase would allow to separate it from competing pathways and to allow an efficient synthesis of thymidylate, which is converted into dTTP for DNA synthesis [7]. This process is further optimised through substrate channeling, the direct transfer of a reaction product between functional consecutive enzymes in multienzyme complexes or between domains in multifunctional enzymes. This process avoids not only the competition with other enzymes and enzyme complexes, but also protects instable intermediate reaction products and increases the re- 


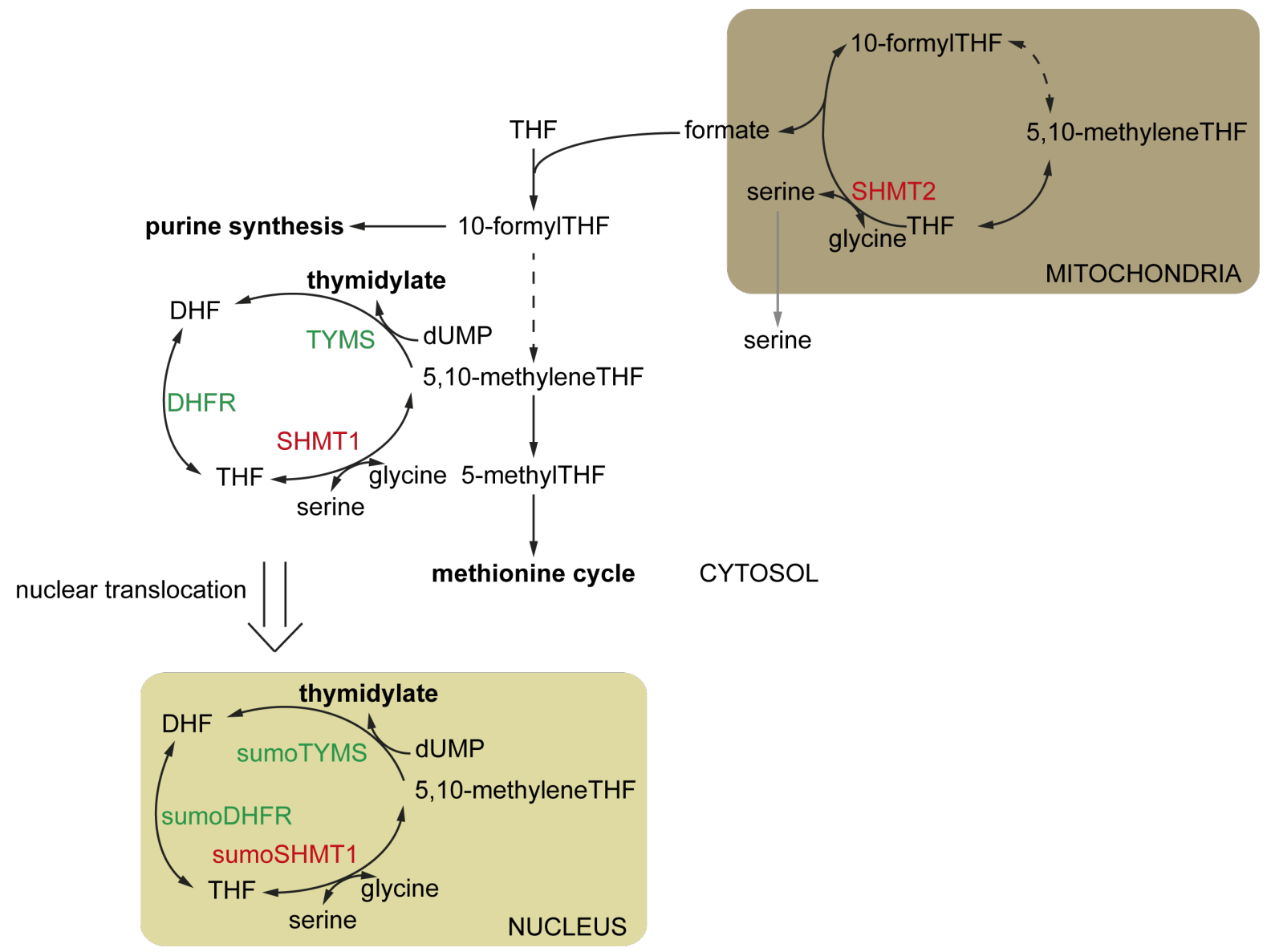

Figure 6: The folate-mediated one-carbon metabolism is compartmentalised in the cytoplasm, mitochondria and nucleus. Cytosolic one-carbon metabolism contributes to the de novo synthesis of purines and thymidylate and is involved in the methionine cycle. Mitochondrial one-carbon metabolism generates formate as one-carbon unit for cytosolic onecarbon metabolism. Upon SUMOylation the thymidylate pathway consisting of SHMT1, DHFR and TYMS is translocated into the nucleus. Only enzymes of the thymidylate pathway are indicated. Direct conversions are marked by solid black arrows, conversions with non-indicated sub-reactions are depicted as dashed black arrows. Transport of serine from mitochondria into the cytosol is marked by grey arrow. Purine synthesis and methionine cycle consist of multiple enzymatic activities. Modified from Anderson et al., 2009 [9]. 
action velocity, because diffusion of reaction products is circumvented [85]. It was shown that human cytosolic SHMT and TYMS physically interact with each other, which could allow substrate channeling [171]. In some species, such as Plasmodium falciparum the thymidylate pathway enzymes DHFR and TYMS evolved to a bifunctional dihydrofolate reductase-thymidylate synthase, which performs substrate channeling for DHF [116]. TYMS transforms dUMP into thymidylate and 5,10-mTHF into DHF, which is remethylated into THF by DHFR. Studies carried out by Stover and coworkers revealed that also SHMT2 participates in the nuclear de novo thymidylate biosynthesis. They could show that the shmt2 gene encodes two isoforms through alternative promoters, one localised in the mitochondria (SHMT2) and one cytoplasmic / nuclear form (SHMT2 $\alpha$ ) $[9,62,65,145]$. SHMT2 $\alpha$ is functionally redundant with SHMT1 in the thymidylate synthesis pathway and is responsible for the residual activity of the pathway in shmt1/mice [9].

The plant genome also carries several genes for shmt. The isoforms are localised, similar to mammals, in the cytoplasm [80] and in the mitochondria [19, 97, 145]. Additionally also a chloroplastic isoform could be identified [161]. Mitochondria are the most active compartment for SHMT function in plants (about $50 \%$ of the total cellular activity) [22].

\subsection{4 shmt mutations characterised in other organisms}

Controlled dTTP synthesis is essential both for DNA replication and genome stability $[8,129]$. Defective thymidylate synthesis results in misincorporation of deoxyuridine into the DNA, which destabilises the genomic integrity [25]. Although $S H M T 1^{-/}$mice are viable and fertile, they have an increased risk for intestinal cancer as well as neural tube defects (NTD) [16, 96]. NTDs are also common birth defects in human. They result from a failed neurulation during early embryonic development. Although heterogeneous genetical and environmental factors are responsible, NTDs can often be prevented by maternal supplementation with folic acid. This indicates a major role of the folate metabolism in neural tube closure [17]. Recently it was shown that affecting thymidylate biosynthesis is responsible for the NTD phenotype [16].

A human variant of SHMT1 carrying a point mutation at amino acid position 474 from leucine to phenylalanine causes a higher risk of cardiovascular diseases and lung cancer $[91,119,169]$. It was shown that SUMOylation is affected in this mutant form of SHMT1, which leads to a defective nuclear translocation in $\mathrm{S}$ phase [9].

The human Smith-Magenis syndrome (SMS) is caused by an interstitial deletion of chromosome 17 in band p1.2 [140]. This disease has a complex phenotype of growth and developmental delay, anomalies of the face, and unusual behaviour [67, 140]. Several 
genes have been identified, which are affected in this chromosomal deletion and contribute to the complex phenotype $[34,36,183]$. shmt1 was found to be one of these genes [51].

\subsubsection{SHMT in Drosophila}

Flybase (flybase.org), the database of Drosophila genes and genomes, lists the gene for shmt as CG3011. It is mentioned as a maternally provided transcript, which is degraded in the blastoderm stage (FlyExpress RNA in situ hybridisation data). The protein is predicted to be mitochondrially localised based on its sequence and/or structural similarity with UniProtKB:P34897, the human SHMT2 protein [130].

shmt was identified in a screen for ethanol-regulated genes [83]. CG3011 ${ }^{\mathrm{NP} 6075}$ flies have an increased ethanol-induced hyperactivity when compared to Wild type (WT). The identification of two genes involved in serine synthesis indicated that this pathway may negatively regulate hyperactivity induced by ethanol as well as it could promote sensitivity against ethanol sedation. During this work, shmt was identified in a screen for mutations affecting neural development [177]. Additional mutations were isolated in the X chromosome saturation screen of the Bellen laboratory [71].

The X238 mutation, on which I was working, was identified in an Ethyl methanesulfonate (EMS) mutagenesis screen for mutants with defects in oogenesis and early embryogenesis performed in the group of Prof. Dr. Nüsslein-Volhard [164]. The mutation was mapped by Prof. Dr. Großhans and sequenced by Kristina Hänecke.

\subsection{Aim of the work}

This work was subdivided into two projects. The focus of the main project was to study the role of SHMT in the early embryonic development of Drosophila. Two main questions were addressed in this study:

1) Why does cellularisation not occur in X238 mutants?

As depicted in the first section of chapter 2, the cellularisation markers Slam, Dlg and Dia are expressed and localised, but there is no invagination of a cellularisation furrow. Therefore, we wanted to find out, how SHMT influences the cellularisation process.

2) Why does the syncytial development of X238 mutants stop already after 13 instead of 14 cycles?

As described in section 2.2.4, X238 mutants stop their syncytial cell cycle program one cycle earlier than WT. We wanted to analyse, in which way SHMT interacts with the cell cycle progression. 
The second project dealt with the analysis of centrosomal fluctuations in the syncytial interphases of Drosophila embryos. The aim was to establish an assay to measure the fluctuations of centrosomes. With this we wanted to analyse the influence of the cytoskeleton as well as the microtubule motor Kinesin-1 on centrosomal fluctuations. 


\section{Results}

\subsection{The $X 238$ mutation is located in the gene encoding for the enzyme Serine hydroxymethyltransferase}

\subsubsection{X238 creates a premature stop codon in the shmt gene}

The mutant X238 was identified in an EMS mutagenesis screen for early embryonic phenotypes [164]. Here, it was screened for mutants with defects in presyncytial, cellularisation, or gastrulation stage. X238 germline clones showed a defective furrow invagination during cellularisation stage with $100 \%$ lethality. The mutation was mapped by Prof. Dr. Jörg Großhans (Fig. 7). He crossed the mutant line to lines with large duplications and deficiencies. Thereby he identified that all duplication lines, which complemented the lethality phenotype also complemented the cellularisation phenotype. This indicated that both phenotypes could be due to a single mutation. Further mapping crosses with lines containing smaller deficiencies and duplications allowed to restrict the genomic area with the mutation to a genetic region between the genes act5c and $f_{s}(1) M 3$ spanning 11 genes. This genomic region was sequenced by Kristina Hänecke, which revealed a single point mutation in the gene CG3011 encoding the enzyme Serine hydroxymethyltransferase (SHMT) leading to a premature stop codon in its $\mathrm{C}$ terminus.

\subsubsection{X238 mutant embryos can be rescued by introduction of a functional shmt gene}

In order to prove that the mutation of the shmt gene was responsible for the observed phenotypes, flies carrying genomic rescue constructs were generated. The genomic region spanned by the rescue construct is depicted in Fig. 8. The shmt gene encodes three transcripts (RA, RB, and RC) from alternative promoters. Whereas the transcripts $\mathrm{RB}$ and $\mathrm{RC}$ translate the same peptide sequence $\mathrm{PB}, \mathrm{PA}$ originating from RA contains additional 60 amino acids (aa) at the $\mathrm{N}$ terminus, which could contain a putative mitochondrial localisation sequence.

The full genomic rescue construct (SHMT-rescue) contained the depicted genomic region with the WT shmt gene. Crossing SHMT-rescue flies to X238-heterozygous flies led to a full rescue of both the blastoderm and the lethality phenotypes as well as it restored 


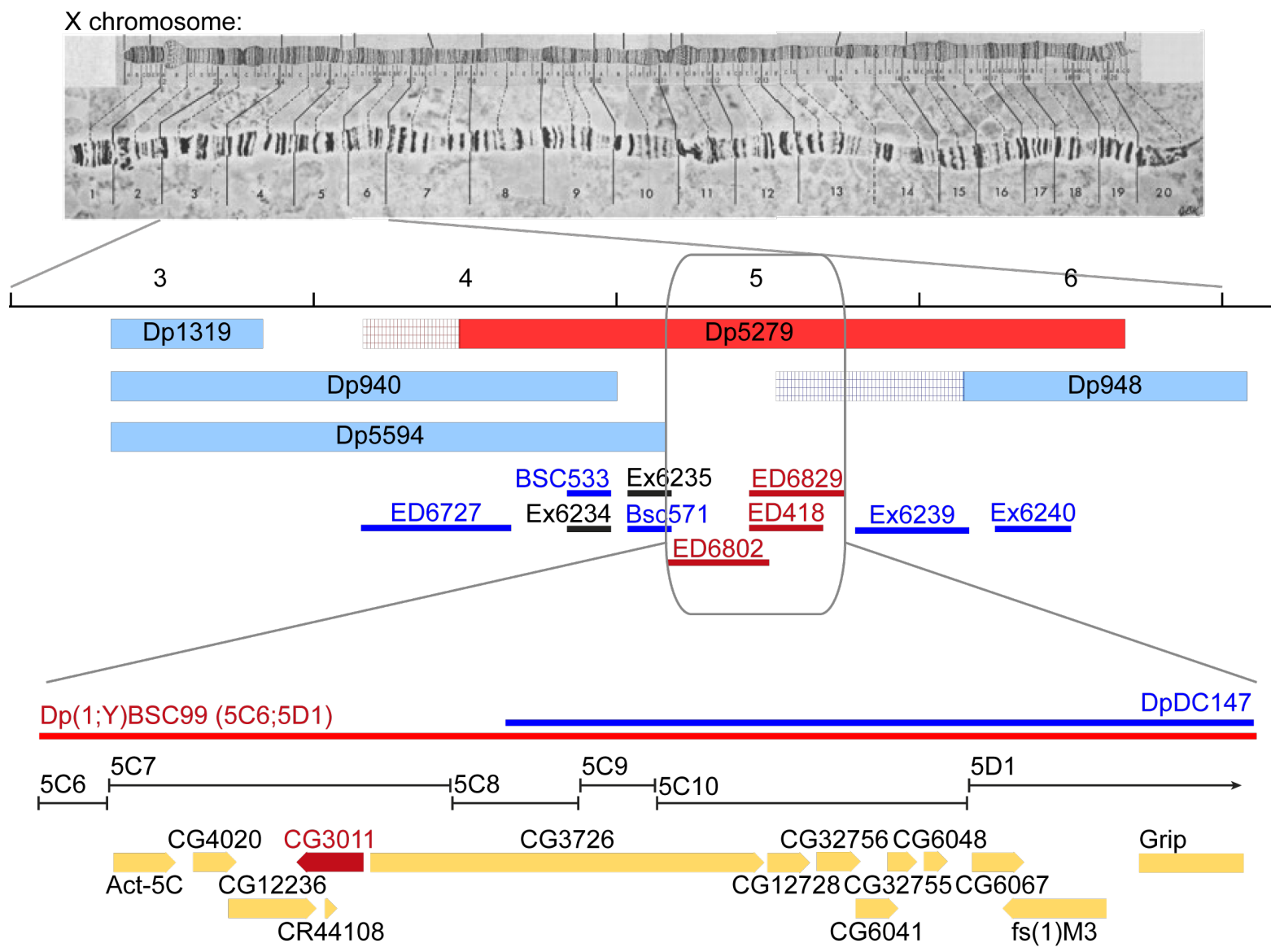

Figure 7: Mapping of the X238 mutation. The X238 lethality was mapped by complementation with large duplications and deficiencies. Complementing duplications also complemented the germline clone phenotype. The position of X238 was restricted to a genomic region consisting of 11 genes between $a c t 5 c$ and $f_{s}(1) M 3$. Sequencing analysis revealed a single point mutation in the $\mathrm{C}$ terminus of the gene CG3011 resulting in a premature stop codon. Cytological positions, gene names, duplication and deficiency fly lines are indicated. Following duplication lines were used to map X238: Dp1319, Dp940, Dp5594, Dp5279, and Dp948; Dp5279 was able to complement the X238 mutation. Deficiency lines in the region of Dp5279 used: ED6727, Bsc533, Ex6234, Ex6235, Bsc571, ED6802, ED6829, ED418, Ex6239, Ex6240; ED6802, ED6829, ED418 were not able to complement X238. All lines were obtained from Bloomington. Mapping and sequencing were carried out by Prof. Dr. Jörg Großhans and Kristina Hänecke, respectively. 


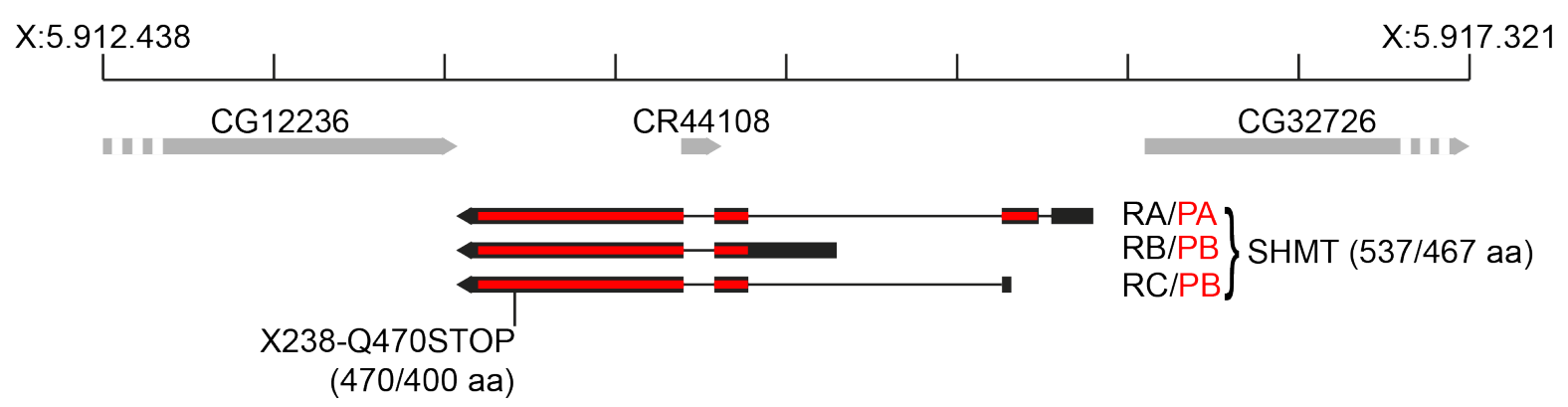

Figure 8: Scheme of the genomic region surrounding the shmt gene with the position of the X238 mutation indicated. Genomic region X:5.912.438 to X:5.917.321 is indicated. In black / red the shmt gene is shown, with its three transcripts (RA, RB, and RC) in black boxes, introns in black lines, and the peptides (PA and PB) in red boxes. Neighbouring genes are marked in grey. CG12236 and CG32726 locate partially in this region. Position of the mutation X238 is indicated. The whole region was cloned as full genomic rescue construct SHMT-rescue.

fertility to WT level (Tables 8, 9, and 10). This confirmed X238 as a mutant of the shmt gene.

\subsection{Phenotypic description of the shmt mutants $\mathrm{X} 238$ and M281R}

In the lab the X238 mutation was kept on two different chromosomes with different flippase recognition target (Frt) sites (X238FRT19A and X238Frt18E). A second allele of shmt (M281R) was isolated in a screen for mutations affecting the nervous system performed by Yamamoto et al., 2014 [177]. Both mutations caused a lethality phenotype during the syncytial blastoderm development in embryos from germline clones.

\subsubsection{The $\mathrm{X} 238$ mutant is not able to cellularise, although cellularisation markers are localised}

In order to understand the development of X238 mutant embryos, time-lapse imaging using differential interference contrast (DIC) optics was performed, which revealed a defect in late blastoderm stage (Fig. 9A). WT embryos undergo 13 rounds of fast syncytial divisions. In cycle 14, the cell cycle progression is paused and cellularisation takes place. In this process, which lasts appr. one hour, the cellularisation furrow invaginates between the nuclei and encloses them in a single layer of cells, forming the cellular blastoderm. In X238 mutant embryos, the cell cycle pause was already established in cycle 13 (for detailed description and analysis of the cell cycle phenotype, refer to section 2.2.4). At appr. 20 to $40 \mathrm{~min}$ in interphase 13, the nuclear morphology became misshaped and the 
regular nuclear arrangement got disturbed (Fig. 9B). The nuclei started to fall into the embryonic core, a process which is called "nuclear fallout". This was defined as the "early defect phase". Only a few minutes later a wave-like movement transported the remaining nuclei into the interior of the embryo. This stage was defined as "late phase defect". In a very late phase defect, the embryonic material accumulated in the anterior half of the egg (Fig. 35: left site, fourth panel). Furrow invagination was not detectable until late defect phase (Fig. 9B, furrow front is marked by arrows). Although the majority of the X238 embryos did not progress into cellularisation (95.8\%, Table 4), some were able to partially or fully cellularise $(4.2 \%)$, which was sometimes accompanied by an additional (partial) cell cycle $(8.3 \%$ and $4.2 \%$, respectively, Table 3$)$. The embryos failed to gastrulate and did not initiate germband extension. The proportional distribution between the detected phenotypes varied between the experiments (refer to Tables $3 / 4$ : "X238Frt19A" and 17/18: "uninjected" for comparison).

A

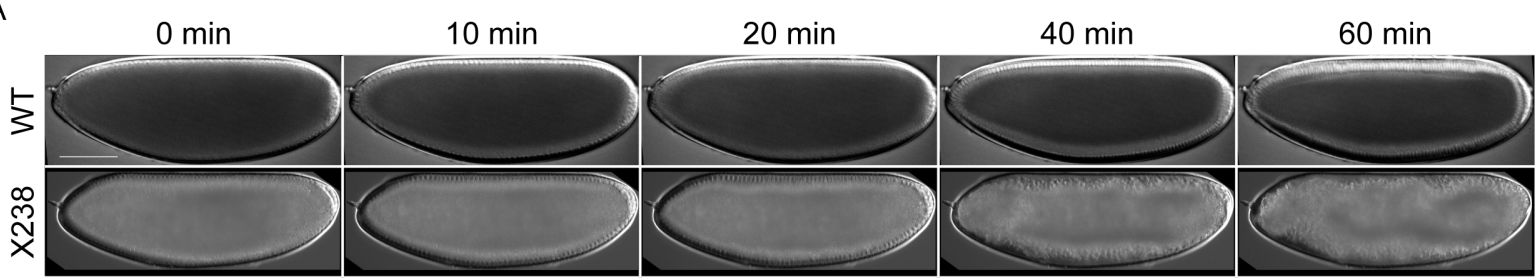

B

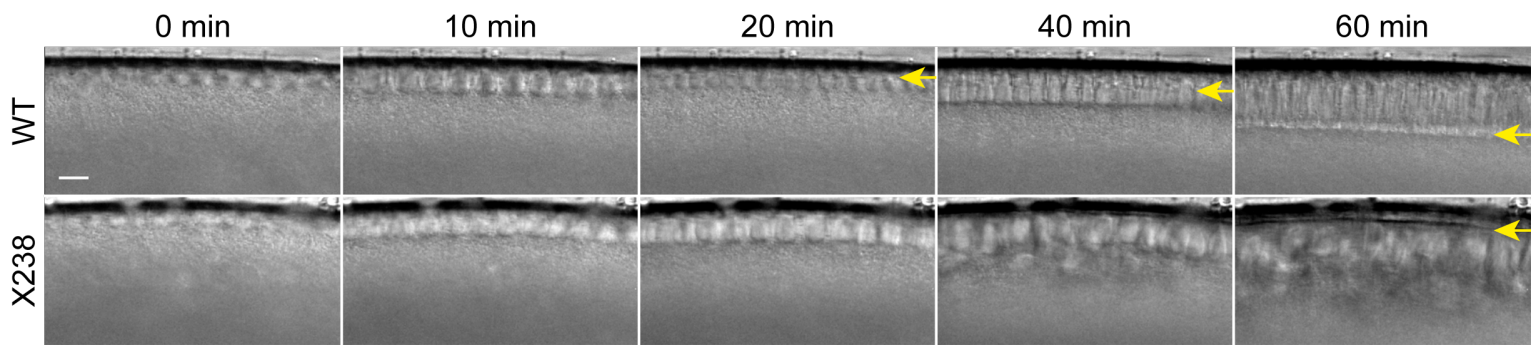

Figure 9: Depletion of SHMT disrupts invagination of the cellularisation furrow. WT and X238 mutant embryos were imaged by DIC microscopy. (A) Imaging of whole embryos. X238 embryos developed until syncytial blastoderm stage, but were not able to cellularise. Scale bar: $100 \mu \mathrm{m}$. (B) Magnified view on the cortical part of the embryo. Arrows point to the invaginating cellularisation front, which was absent in the majority of X238 embryos. Scale bar: $10 \mu \mathrm{m}$.

To analyse the cellularisation phenotype in more detail, immunostainings against known cellularisation markers were performed. The formin protein Dia mediates nucleation and polymerisation of actin filaments and regulates microtubule stability [35, 77, 115, 167]. Both actin and microtubule remodelling is necessary for progression through the syncytial blastoderm cycles. Dia is localised at the pseudocleavage furrows during syncytial blastoderm and at the furrow canal in cellularisation (Figure 10, left panels). A similar pattern was observed in X238 mutants (Figure 10, right panels). In late defect phase, 


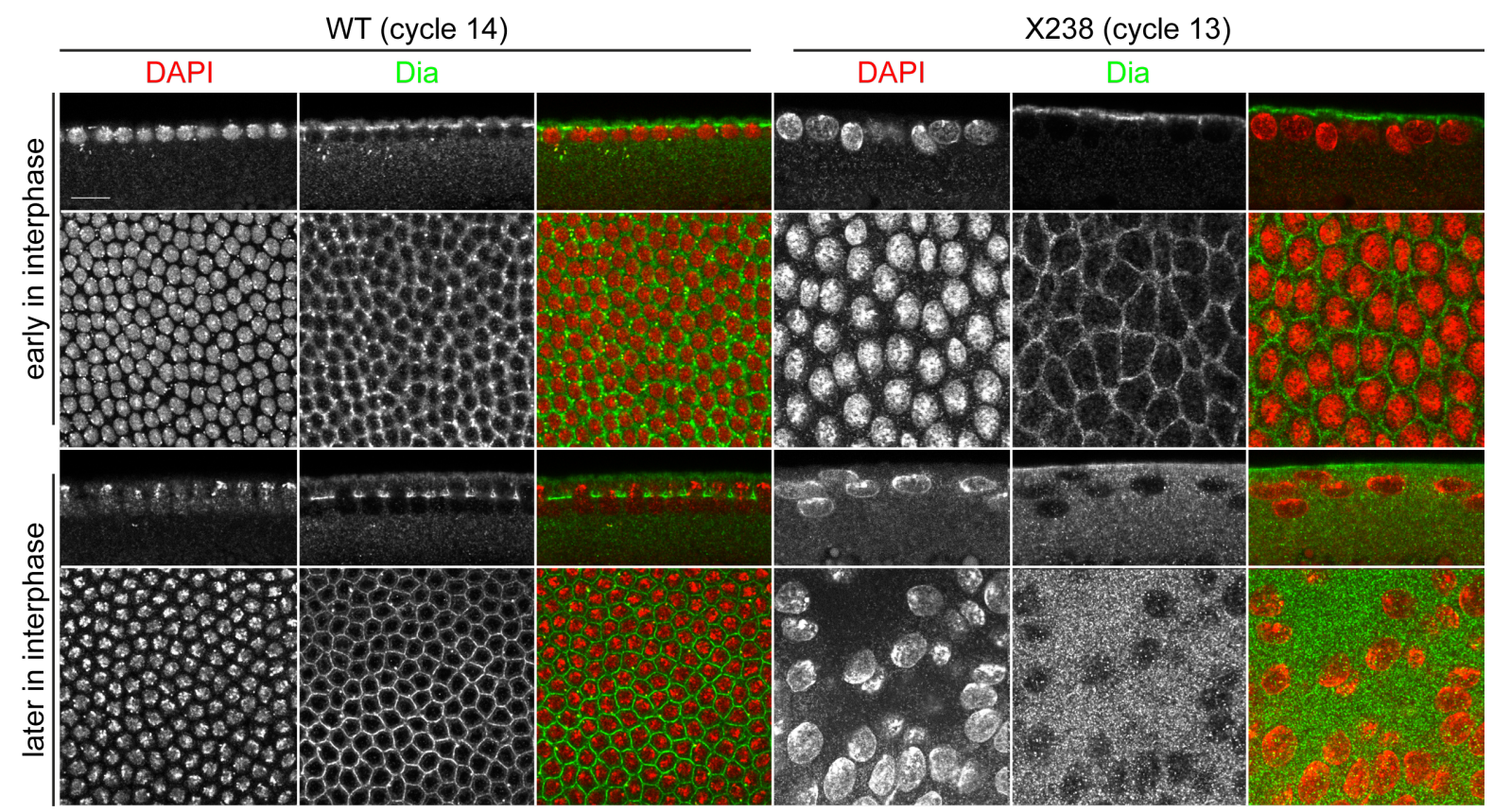

Figure 10: Localisation of the furrow canal marker Dia is not affected in the mutant. Immunostaining of WT and X238 embryos with Dia antibody and the DNA dye DAPI. Dia protein was enriched at the furrow canal during cellularisation. Dia localisation in X238 embryos was comparable to WT during early cellularisation. In the late defect phase the staining became diffuse. Scale bar: $10 \mu \mathrm{m}$.

Dia lost its localisation and the signal became evenly distributed throughout the cortical part of the embryo.

In order to analyse the missing furrow invagination, Slam protein localisation was determined by immunostaining. Slam is required for invagination and compartmentalisation of the cellularisation furrow. In WT it accumulates mainly at the onset of cellularisation, although it is already detectable in previous syncytial cycles (Fig. 11, left panels). Slam localises exclusively to the invaginating furrow canal and basal particles [2]. In cycle 13 of X238 embryos, Slam had a similar localisation pattern as in WT (Fig. 11, right panels). During early defect phase, Slam protein staining diffused, but the pattern remained arranged. In late defect phase, Slam became unlocalised.

To determine the formation of the lateral membrane, immunostainings against the marker proteins Dlg and Sry- $\alpha$ were performed. Dlg is a membrane protein localised at the lateral membrane of the cellularisation furrow (Fig. 12). In X238 Dlg was accumulated at the membrane similar to WT embryos early in cellularisation, before furrow invagination was initiated.

Localisation of the basolateral membrane protein Sry- $\alpha$ differed between WT and X238. In WT, Sry- $\alpha$ protein starts to localise in cycle 12 . There a very weak staining is 

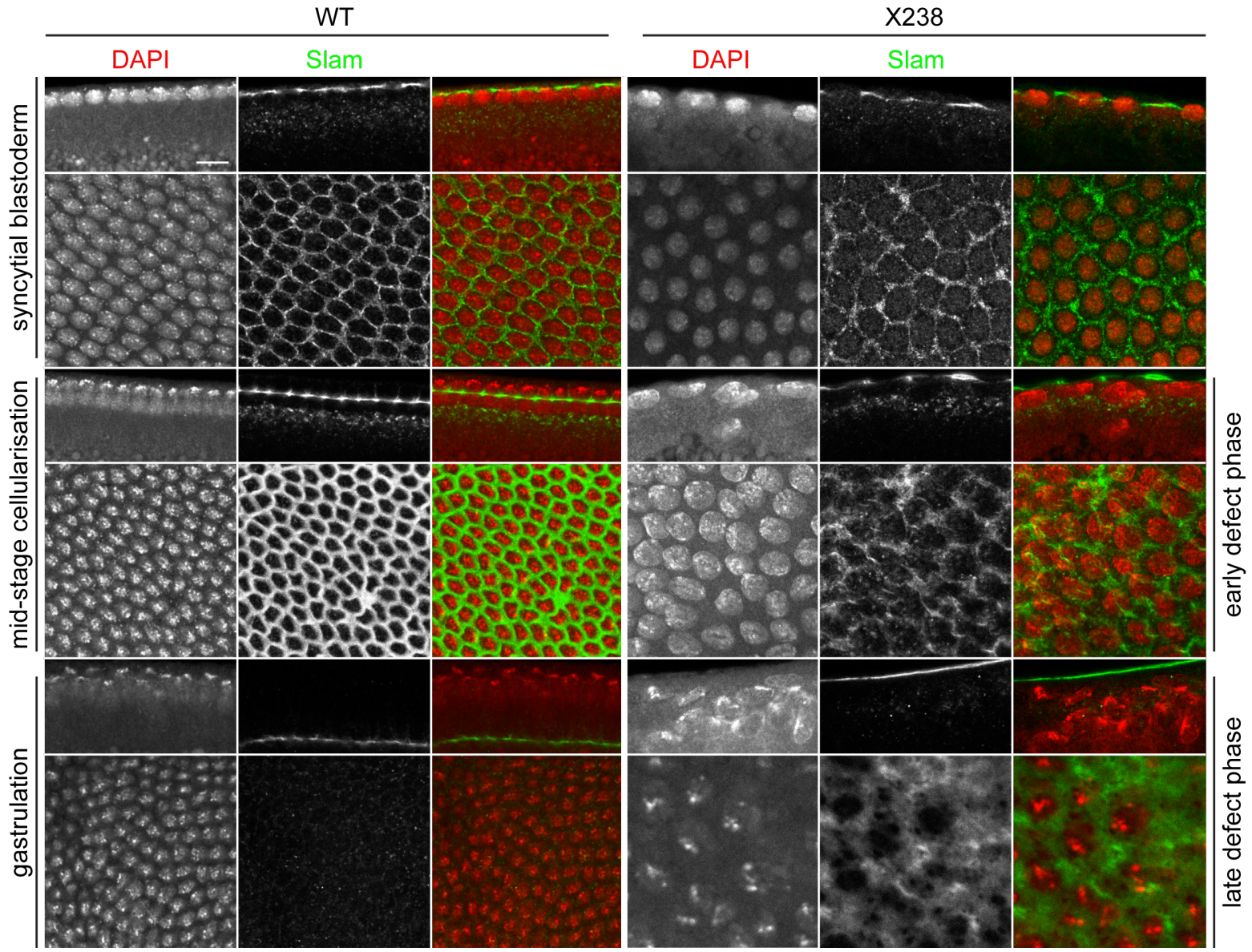

Figure 11: Slam protein is localised at the invaginating furrow canal. WT and X238 mutant embryos were stained with Slam antibody as well as DAPI to label nuclei. Slam localised to the invaginating furrow canal. Although X238 mutants missed furrow invagination, Slam was accumulated at the membrane. In the late defect phase, a localised Slam signal was not detectable. Scale bar: $10 \mu \mathrm{m}$. 


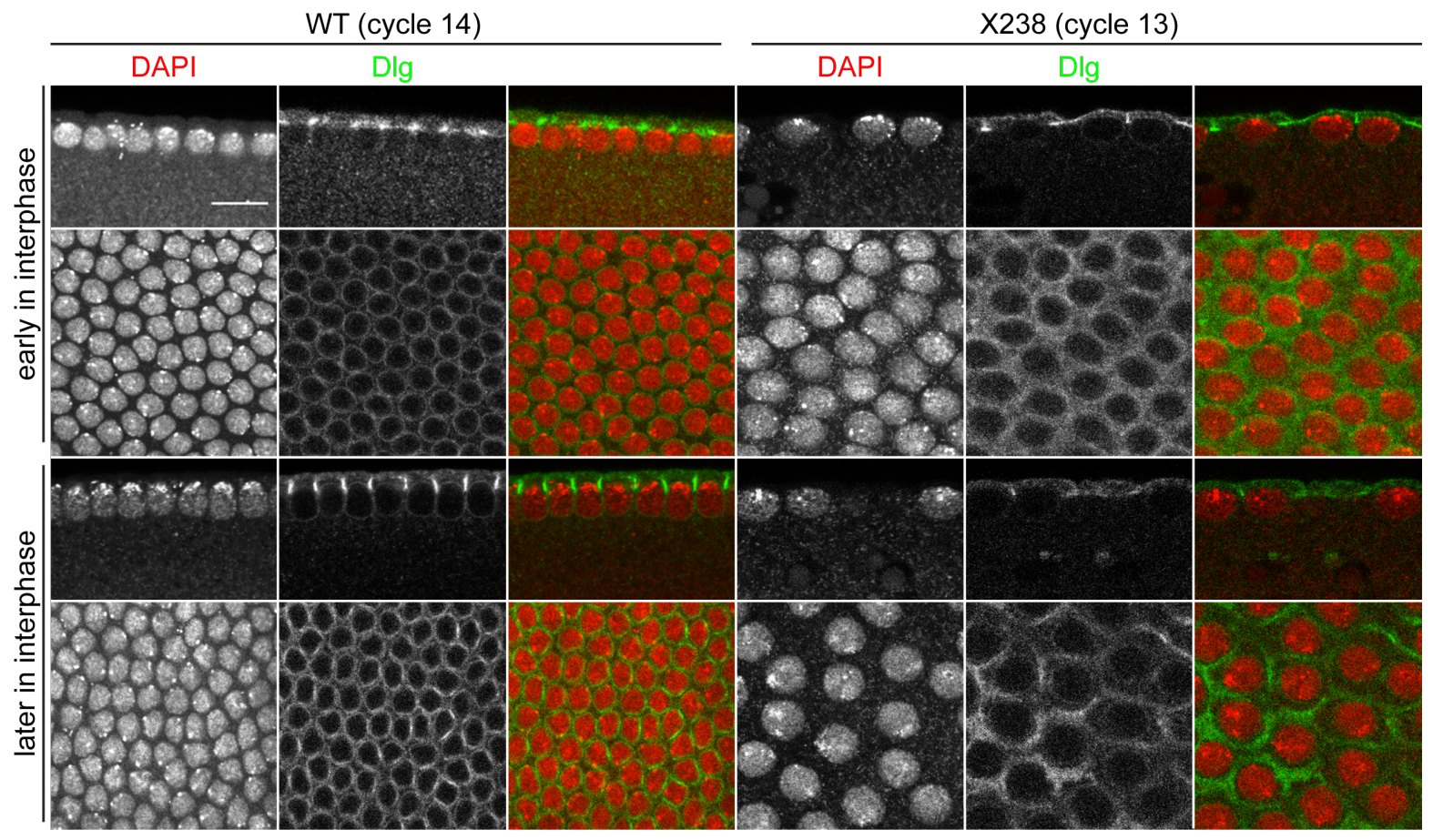

Figure 12: Localisation of Dlg, a marker for the lateral membrane, is not altered in X238 embryos. WT and X238 embryos were immunostained with Dlg antibody and DAPI, which labels DNA. Dlg was accumulated at the lateral membrane of the cellularisation furrow. Dlg localisation in the mutant was comparable to that in WT embryos in early cycle 14 before furrow invagination started. Scale bar: $10 \mu \mathrm{m}$. 


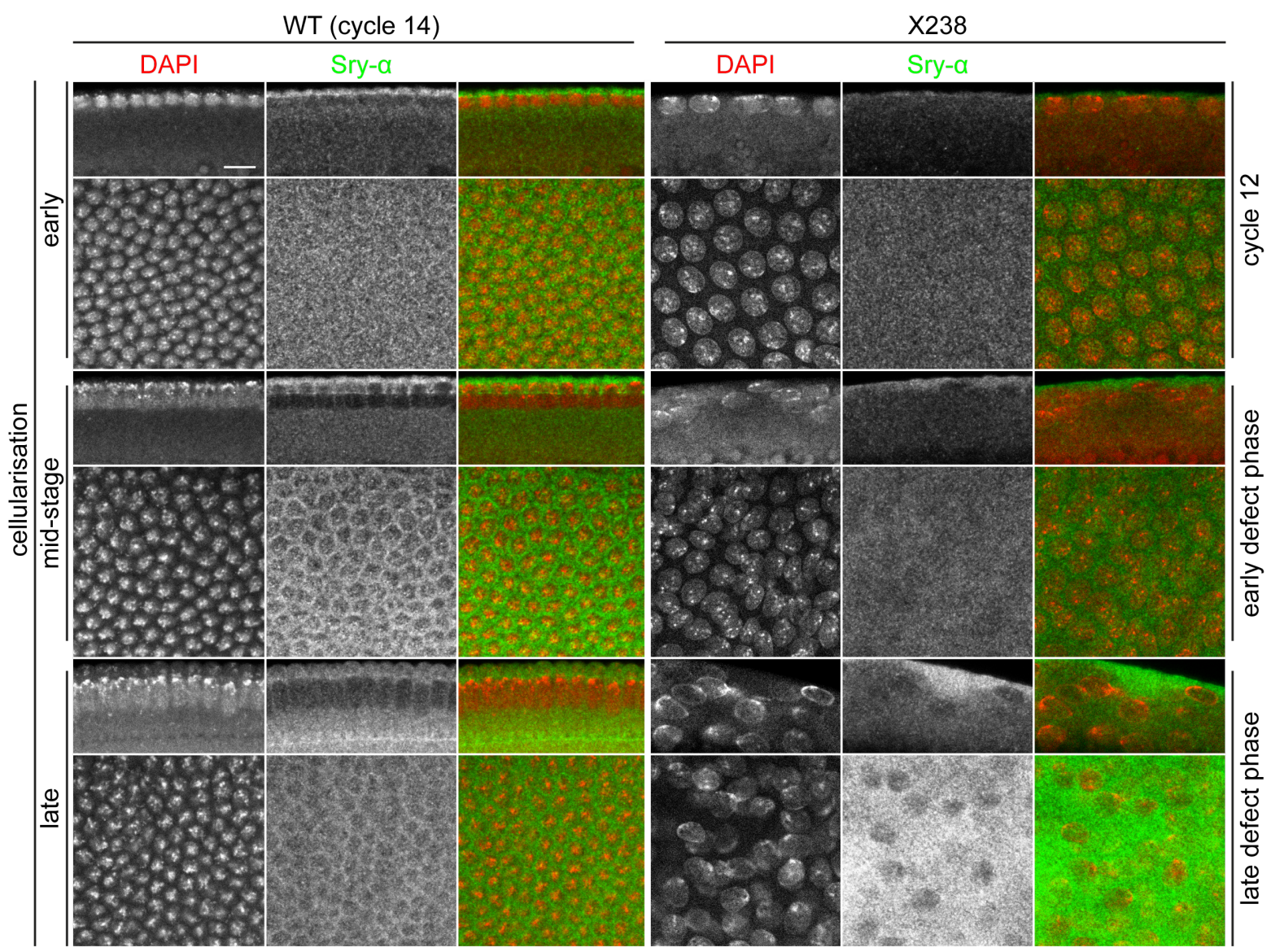

Figure 13: Sry- $\alpha$, a late cellularisation protein, is not localised in X238 embryos. Immunostaining of WT and X238 embryos with an antibody against Sry- $\alpha$ and the DNA marker DAPI. In WT embryos Sry- $\alpha$ accumulated at the apical membrane and the furrow canal at the beginning cycle 14. In X238 embryos this accumulation was not detectable. X238 embryos in late defect phase exhibited an increase in cytoplasmic signal. Scale bar: $10 \mu \mathrm{m}$.

detectable compared to cycle 14, where it becomes strongly increased [133]. At the beginning of cellularisation, Sry- $\alpha$ is enriched at folds and microprojections of the apical membrane (Fig. 13, left panels). During progressing cellularisation, Sry- $\alpha$ becomes enriched at the furrow as well as at the furrow canal, although a weak cytoplasmic signal is detectable too. At the end of cellularisation Sry- $\alpha$ signal becomes cytoplasmic and disappears in gastrulation stage. In X238 Sry- $\alpha$ signal intensity was decreased, but in late defect phase the signal became brighter (Fig. 13, right panels).

In summary, early cellularisation markers were present in the mutant, whereas the later cellularisation marker Sry- $\alpha$ was not. This raised the question if patterning formation occured in X238. In WT embryos localisation of gap and pair-rule genes is established during cellularisation [139]. At the beginning of cycle 14 Even skipped (Eve) accumulates in the posterior two-thirds of the embryo [60]. Later in interphase 14 expression pattern 


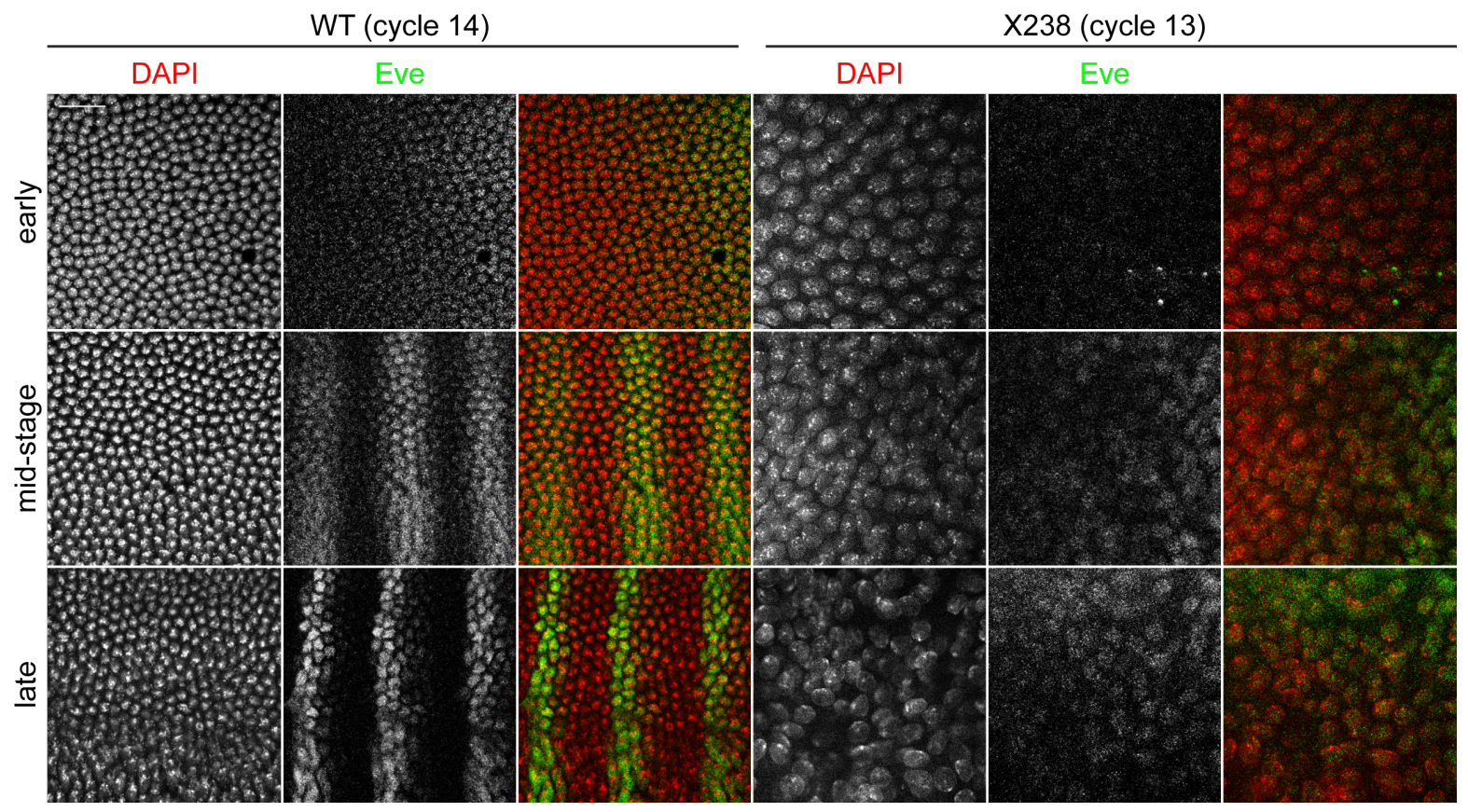

Figure 14: Protein localisation of Eve reflects the expression pattern of WT embryos in early cellularisation. WT and X238 embryos were immunostained with Eve antibody and the DNA marker DAPI. At the beginning of cycle 14 Eve was mainly located in the posterior two-thirds of the embryo. During cellularisation the stripe-like staining pattern was established. X238 embryos exhibited the early posterior staining until late defect phase. Scale bar: $10 \mu \mathrm{m}$.

of Eve changes into seven evenly distributed stripes with sharp boundaries (Fig. 14, left panels). X238 mutants early in cycle 13 did not show an elevated Eve signal (Fig. 14, right panels). In defect phase it accumulated in a similar fashion as in early interphase 14 of WT indicating that pattern formation is initiated in X238 mutant embryos.

\subsubsection{SHMT reduction in clones does not affect cell proliferation}

SHMT is a central enzyme of the one-carbon (C1) metabolism, which is essential for synthesis of numerous compounds, including lipids and purines [102]. SHMT is a cell essential enzyme necessary for proliferation. X238 mutant embryos, which showed a strong reduction of SHMT in Western blot analysis and immunostainings (refer to section 2.5 for detailed analysis of SHMT presence and localisation), developed until late syncytial blastoderm stage. Therefore we asked, if the strong hypomorphic X238 mutation has an effect on cell proliferation in flies. We wanted to know, if SHMT has a cell autonomous function or if reaction products of the catalysed or subsequent reactions can be transported between cells. To test this, we used imaginal disc and ovary cell proliferation as marker for this functional analysis. Homozygous mutant clones were induced in larval and adult tissue and their proliferation rate was analysed. This was achieved by crossing 
female flies carrying the mutant or a control $\mathrm{X}$ chromosome containing an Frt site to flies with an X chromosome containing a gfp gene coupled to a nuclear localisation signal (nls-gfp) and the same Frt site as well as a flippase gene under a heat shock promoter (hs- $f l p)$. Flippase mediated recombination, activated by heat shock, resulted in a similar number of clone cells, which were depleted of $g f p$ and homozygous for the mutant or control chromosome, and twin clone cells with two copies of $g f p$. Clones of the WT con-

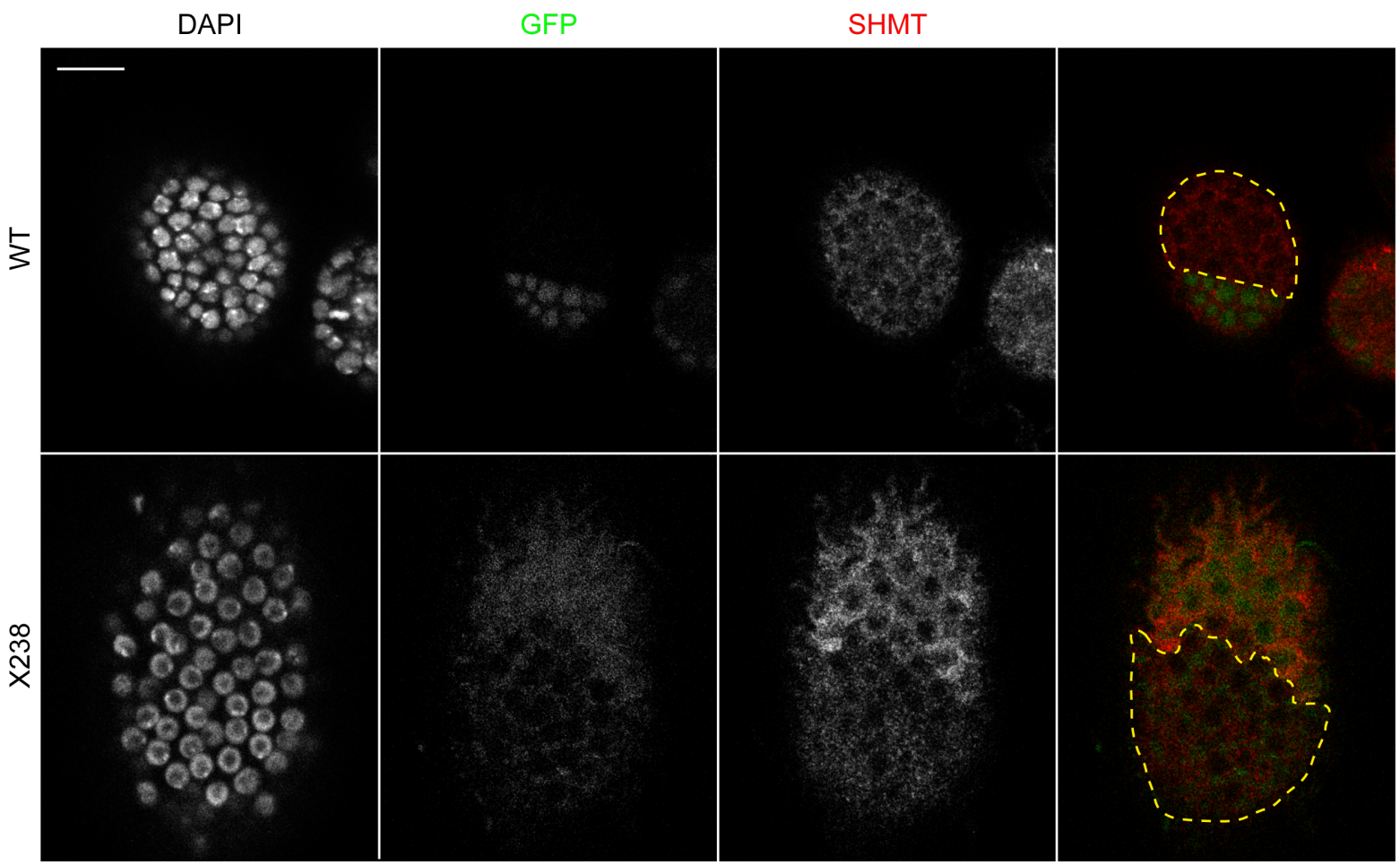

Figure 15: Induction of shmt-depleted clones in follicle epithelium does not interfere with proliferation. Induction of cell clones, which were homozygous for X238 or control, was performed in adult flies and their proliferation was analysed in the follicle epithelium by staining with DAPI and GFP booster. Before activation of flippase all follicle epithelial cells had the genotype: $\frac{\mathrm{X} 238}{\text { nls- } g f p}$ (1xGFP signal). After flippase-mediated recombination and subsequent proliferation cell clones either homozygous for X238/control (no GFP signal) or nls- $g f p$ (2xGFP signal) were formed. Ovaries were extracted 3 days after clone induction. $g f p$-depleted clones (encircled by dashed line) containing multiple cells, which were comparable in size with their corresponding twin clones, were formed both in WT and X238. Scale bar: $10 \mu \mathrm{m}$.

trol in the follicle cell epithelium and the imaginal discs consisted of multiple cells and were of similar size as their corresponding twin clones (Fig. 15 and 16). In X238 follicle cell epithelium, multicellular clones depleted for shmt were formed. The shmt depletion was confirmed by loss of localised GFP as well as SHMT signal (Fig. 15, clones are encircled by a dashed line). These results indicated that either the remaining amount 
of functional SHMT protein in the hypomorphic mutant is sufficient for proliferation or that SHMT has a cell non-autonomous function. This was confirmed by analysing shmt depleted clones in larval imaginal discs (Fig. 16), where large clones of gfp depleted, X238 homozygous mutant cells were detectable. Their twin clones with two copies of gfp (WT for shmt) were of comparable size.

A

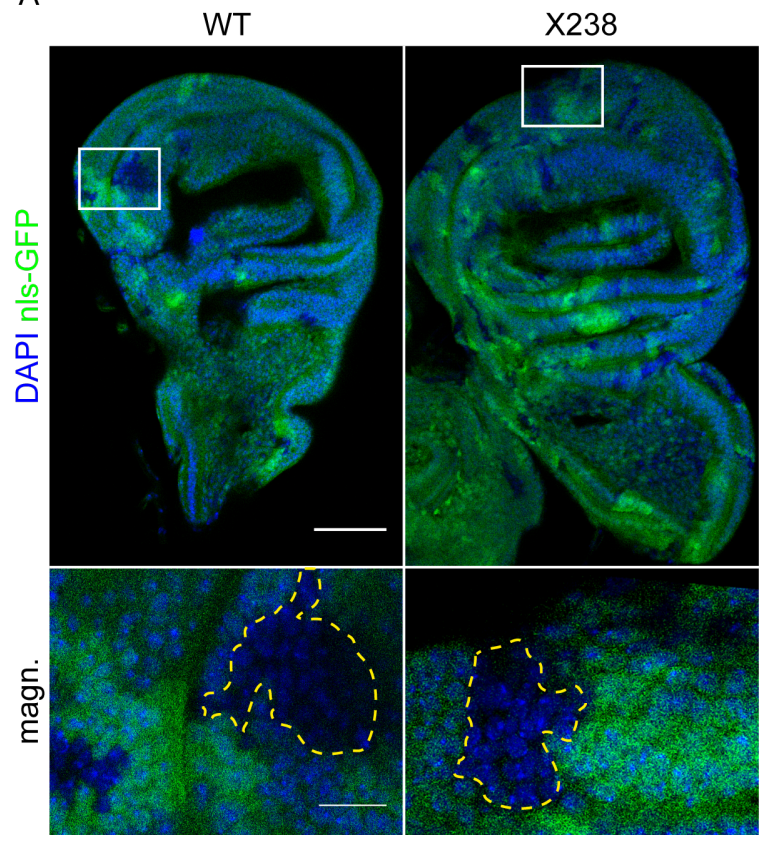

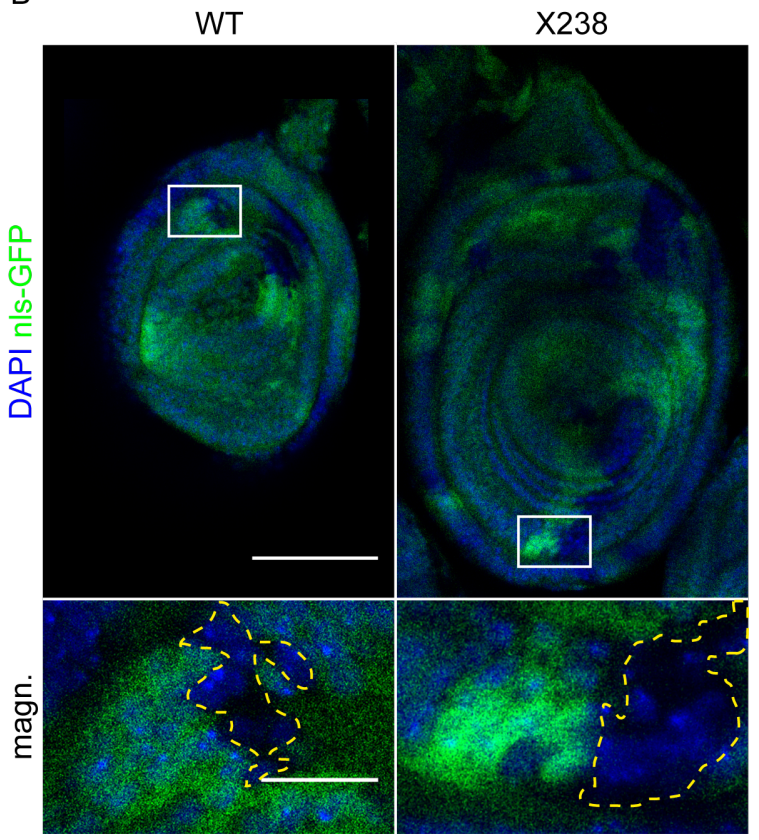

Figure 16: Proliferation is not affected in shmt-depleted clones in imaginal discs Cell clones homozygous for X238 or control were induced in first instar larvae and analysed in imaginal discs by staining with DAPI and GFP booster. They were prepared from late third instar larvae (5 days after induction). shmt-depleted clones (no GFP signal, encircled by dashed line) consisted of multiple cells of a similar size as their twin clones (with two WT copies of shmt, bright GFP signal) both in wing (A) and leg imaginal discs (B). Scale bar: $50 \mu \mathrm{m}$.

\subsubsection{Nuclear morphology becomes disturbed in X238 mutants}

Observation of X238 with light microscopy implicated morphological defects of the nuclei. To analyse this in more detail, immunostainings with the lamina markers Lamin Dm0 (Fig. 17) and Kugelkern (Kuk) (Fig. 18) were performed. Both proteins are localised exclusively to the nuclear lamina. Lamin Dm0 is expressed already in early cycles, whereas Kuk accumulation peaks during cellularisation. In syncytial cell cycles and in early cellularisation, nuclei are round-shaped. As the furrow starts to invaginate, the nuclei become elongated (Fig. 17 and 18, left panels). In X238 the nuclear shape got distorted during interphase 13 (Fig. 17 and 18, right panels). The nuclei did not 
elongate as in WT, they rather expanded laterally. Variable infoldings of the nuclear envelope were observed. Additionally the size of the nuclei increased during cycle 13 of X238 embryos. Beside the defect in nuclear morphology, these experiments revealed a mislocalisation of Kuk protein in X238 (Fig. 18, right panels). Mutant embryos exhibited an uneven staining of Kuk showing nuclei with an intense signal, but also nuclei with a weak as well as without signal. Further investigation in Kuk protein localisation in the mutant is presented in section 2.4.3.

WT

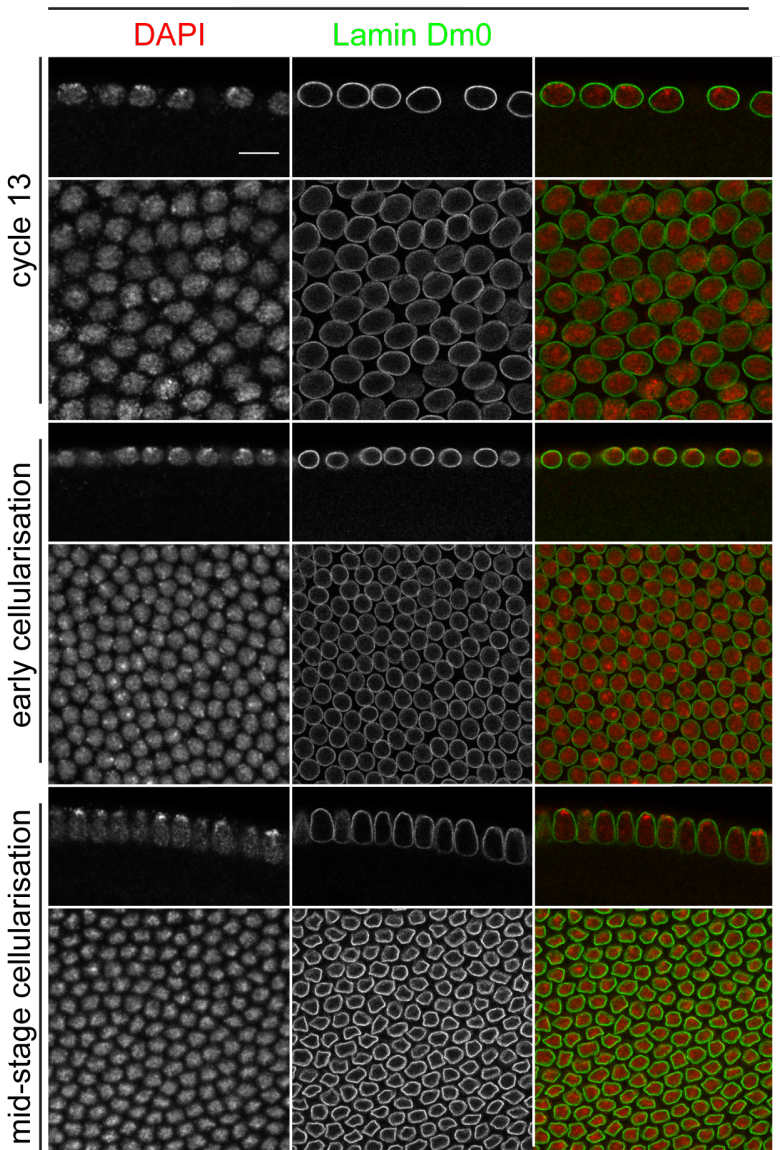

X238 (cycle 13)

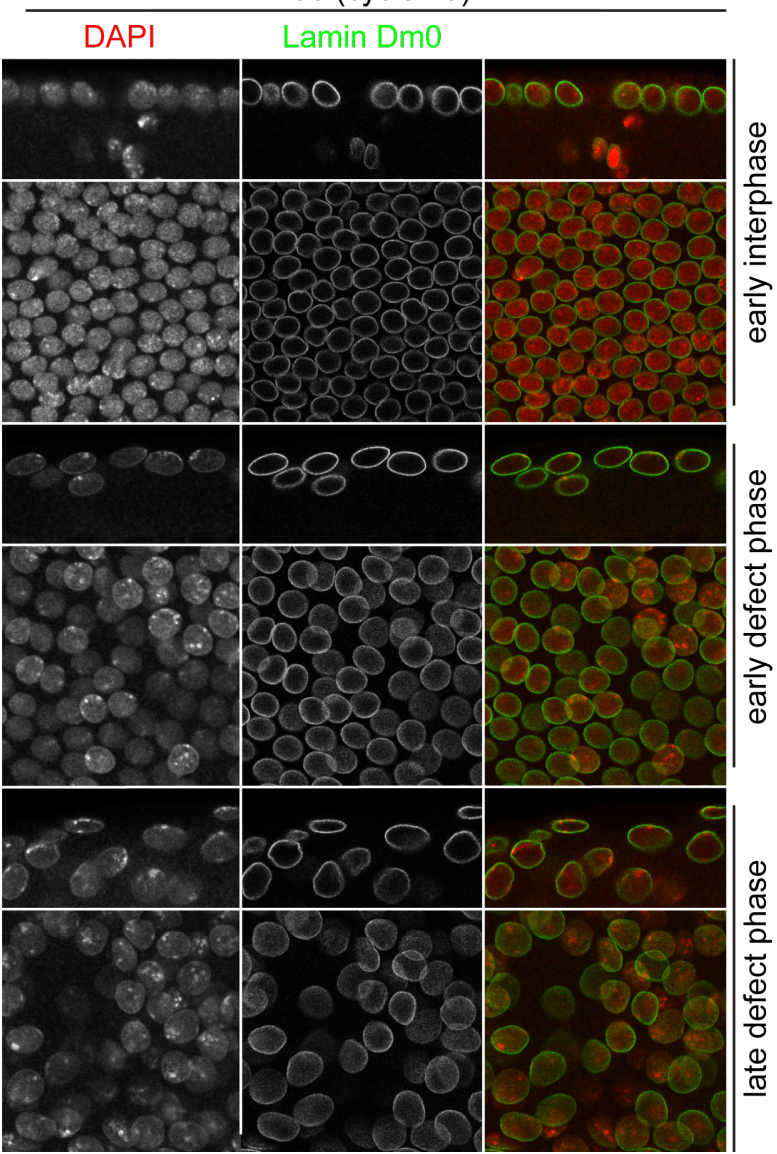

Figure 17: Nuclei become misshaped in X238 mutants during cycle 13. Immunostaining of WT and X238 embryos with Lamin Dm0 antibody to stain the nuclear envelope and DAPI to visualise DNA. In WT the nuclei elongated during the cellularisation process with the majority of heterochromatin localised apically. Nuclei of X238 embryos did not show the apicalbasal elongation, they rather lost their round morphology, became misshaped with infoldings of the envelope and lateral elongation. DNA enriched at the nuclear periphery. Furthermore, the size of the nuclei increased in X238 embryos during cycle 13. Scale bar: $10 \mu \mathrm{m}$. 

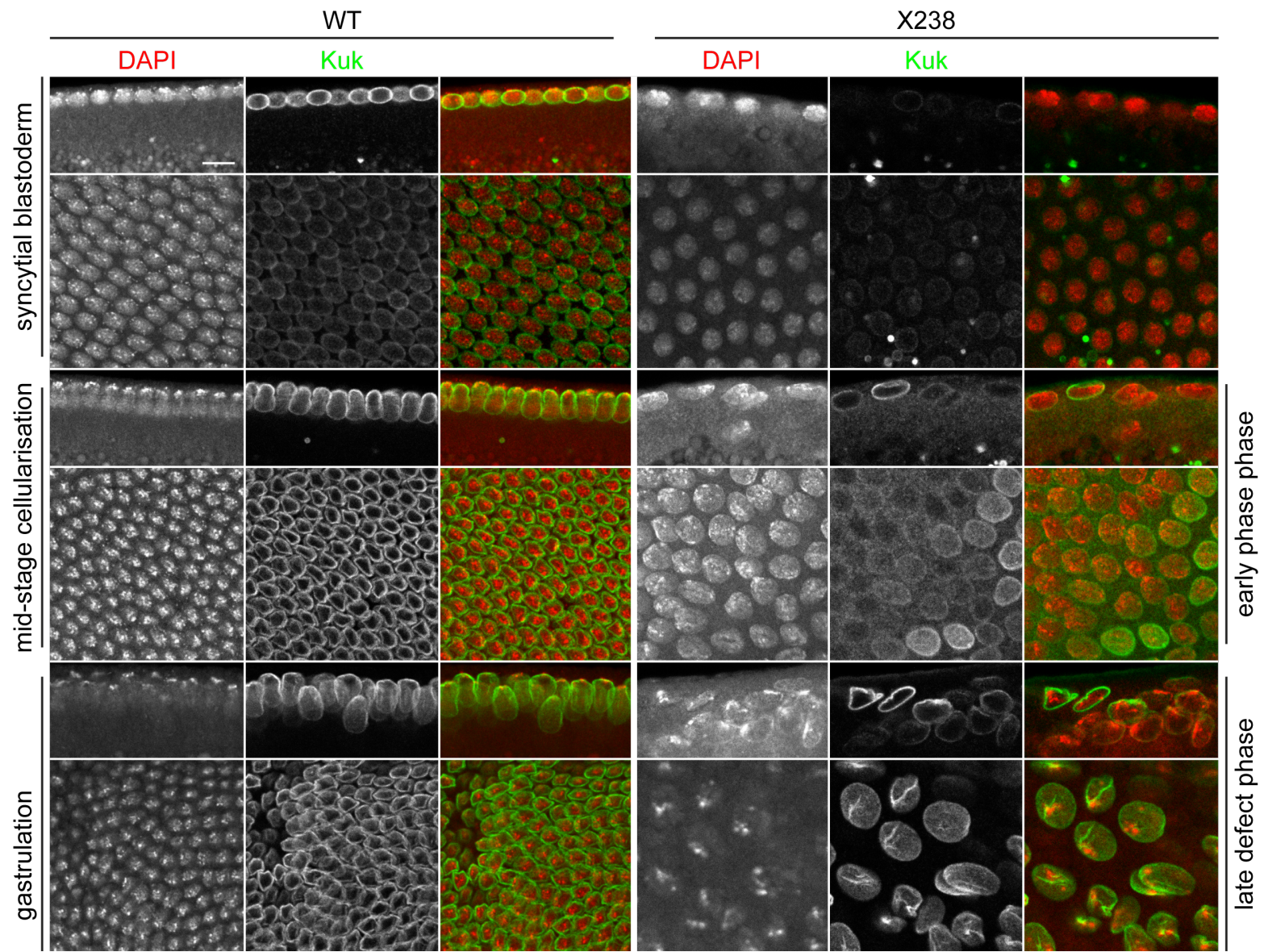

Figure 18: Kuk protein staining in X238 embryos reflects the defect nuclear morphology, although it is not present in all nuclei. WT and X238 embryos were immunostained with Kuk antibody and DAPI as a DNA marker. In WT Kuk protein was accumulated at the nuclear lamina in cellularisation. Although it localised already in syncytial blastoderm, the Kuk signal strongly increased in early cycle 14. The Kuk signal in X238 at the beginning of interphase 13 was comparable to WT cycle 13. The signal intensity increased during early cycle 13 of X238, although it displayed an irregular distribution throughout the embryo. There were nuclei expressing Kuk (in different intensities), but also nuclei without Kuk signal could be observed. Nuclei of both categories showed a similar wrinkling and infolding of the nuclear membrane. Scale bar: $10 \mu \mathrm{m}$.

\subsubsection{Loss of SHMT results in a premature stop of the syncytial cell cycle progression}

X238 mutants stopped their cell cycle progression in cycle 13, one cycle earlier than WT (Fig. 19 and 20A). We wanted to analyse this phenomenon in more detail and wanted to know if X238 exhibits the same gradual increase in syncytial cell cycle length as WT [55]. 


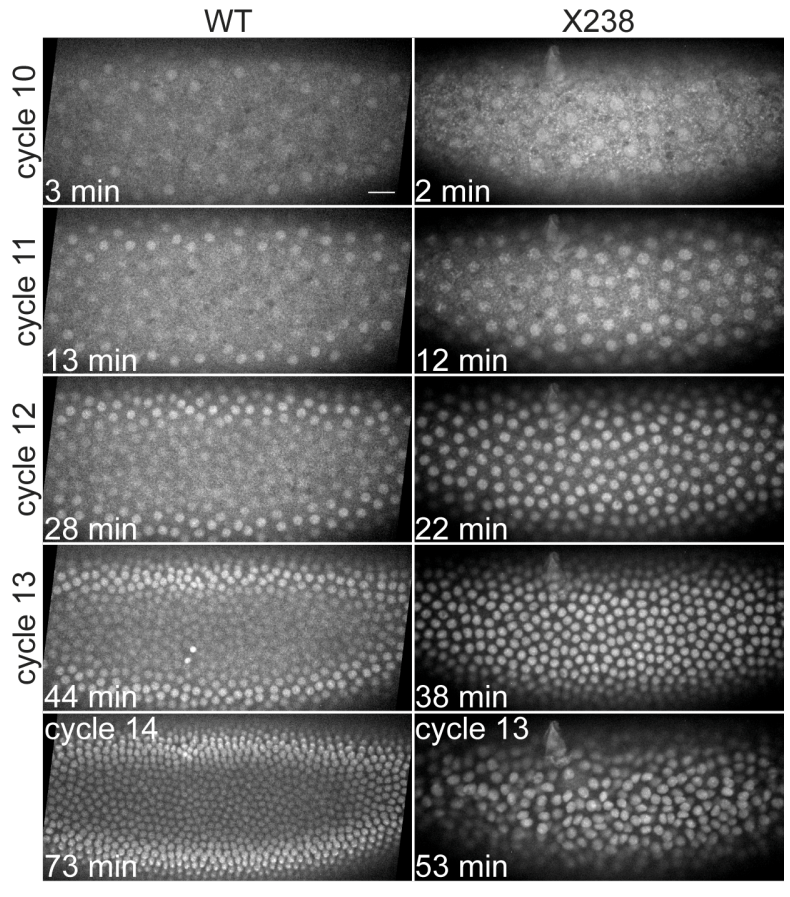

Figure 19: X238 embryos stop cell cycle progression in interphase 13. Images of time-lapse recordings of HisRFP-expressing WT and X238 embryos from cycle 10 to $13 / 14 . t=0$ min defines start of cycle 10 . Nuclear densities of WT and X238 were comparable until cycle 13. After a time interval, in which WT embryos developed to cycle 14, X238 embryos were still in cycle 13 exhibiting morphological defects. Scale bar: $20 \mu \mathrm{m}$.

Table 1: X238 embryos stop their syncytial development in cycle 13.

\begin{tabular}{lcrrcccc}
\hline & & \multicolumn{5}{c}{ Number of nuclei in $\mathbf{5 0}{\mu m^{2} \text { in cycle }}$} \\
\cline { 4 - 8 } Genotype & Cycles & \multicolumn{1}{c}{$\mathbf{n}$} & $\mathbf{1 0}$ & $\mathbf{1 1}$ & $\mathbf{1 2}$ & $\mathbf{1 3}$ & $\mathbf{1 4}$ \\
\hline WT & 14 & 4 & $5.5 \pm 0.5$ & $9.5 \pm 0.5$ & $19.5 \pm 2.8$ & $39.8 \pm 3.3$ & $74.8 \pm 1.3$ \\
X238Frt19A & 13 & 15 & $5.1 \pm 0.9$ & $9.2 \pm 1.6$ & $20.3 \pm 3.1$ & $37.1 \pm 3.6$ & \\
\hline
\end{tabular}

Table 2: Increasing of cell cycle length is similar in WT and X238.

\begin{tabular}{lcrrrrc}
\hline & & \multicolumn{4}{c}{ Cell cycle length (min) } \\
\cline { 4 - 7 } Genotype & Cycles & $\mathbf{n}$ & \multicolumn{1}{c}{$\mathbf{1 0}$} & $\mathbf{1 1}$ & $\mathbf{1 2}$ & $\mathbf{1 3}$ \\
\hline WT & 14 & 6 & $11.8 \pm 0.8$ & $12.5 \pm 0.5$ & $15.0 \pm 0.7$ & $21.8 \pm 0.9$ \\
X238Frt19A & 13 & 12 & $9.7 \pm 0.6$ & $10.6 \pm 1.1$ & $15.4 \pm 1.9$ & \\
\hline
\end{tabular}


A

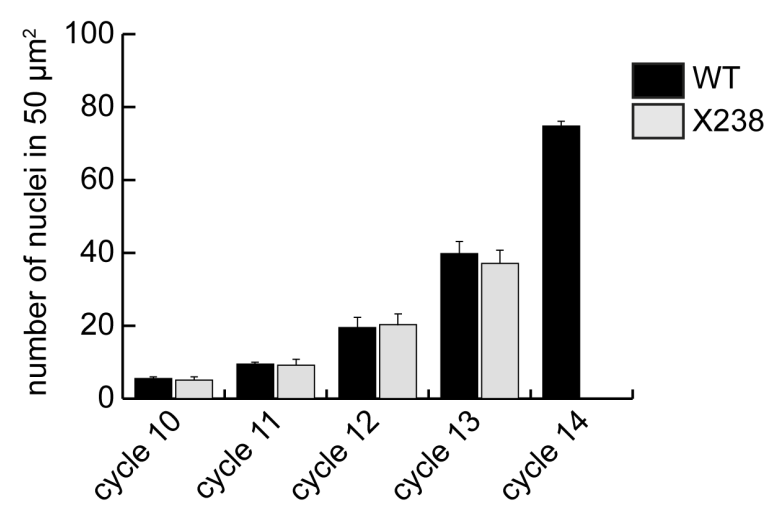

B

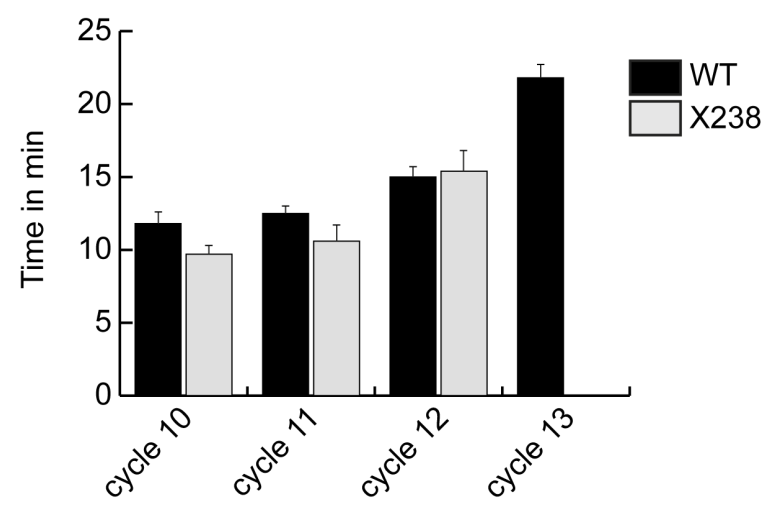

Figure 20: Lengthening in cell cycle duration in X238 is similar to WT, but the cell cycle progression stops already in cycle 13. HisRFP expressing WT and X238 embryos were imaged by time-lapse recordings. (A) Number of syncytial cell cycles was determined by counting the number of nuclei in a defined area of $50 \mu \mathrm{m}$ in the interphases. Comparing these numbers from WT and X238 revealed that X238 mutants stopped their cell cycle progression in cycle 13. (B) Length of cell cycles was measured from the beginning of interphase until the end of telophase. In WT the cell cycle length increased from cycle 10 to 13. A similar prolongation was observed in X238. The bar charts illustrate the results presented in Tables 1 and 2.

Time-lapse imaging of embryos expressing HisRFP was performed. The cell cycle was determined by counting the number of nuclei in a defined space and cell cycle duration was counted from entering interphase to the end of mitosis (Tables 1 and 2, Fig. 20). Cell cycle length increased like in WT indicating that the onset of early zygotic gene expression, which is responsible for this lengthening, occurs in X238.

\subsubsection{Characterisation of a second allele of shmt: M281R}

In 2014 Yamamoto et al. identified a new allele for shmt in an EMS screen for mutants affecting the nervous system [177]. The allele which we called M281R harbours a single point mutation changing the $281^{\text {st }}$ amino acid from methionine into arginine. We analysed its early development by time-lapse imaging. A similar phenotype to X238 was observed, although Western blot revealed that the protein level was not as strongly reduced as in X238 (Fig. 41). Despite of a higher proportion of the embryos being able to undergo mitosis 13 (23.8\% compared to $8.3 \%$ of X238 embryos) and succeeding in cellularisation ( $25 \%$ of M281R and $4.2 \%$ of X238 mutants), the majority of M281R mutants exhibited a premature cell cycle stop in cycle 13 and failed to cellularise $(66.7 \%$ and $70 \%$, respectively; Fig. 21, Tables 3 and 4). 
A

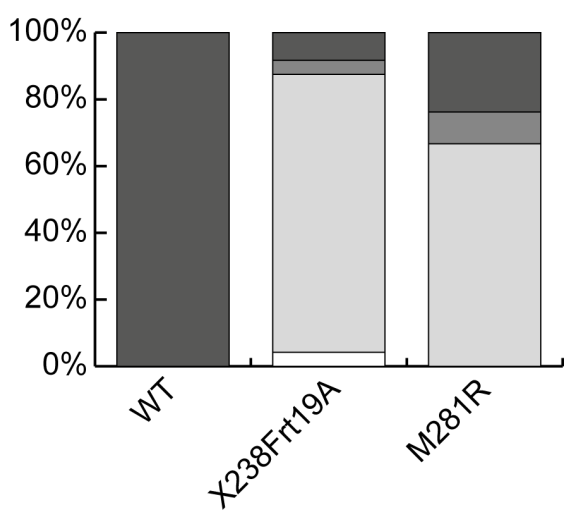

B

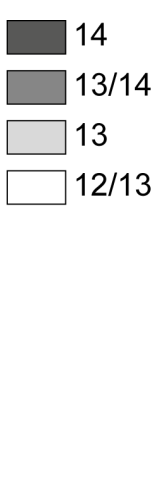

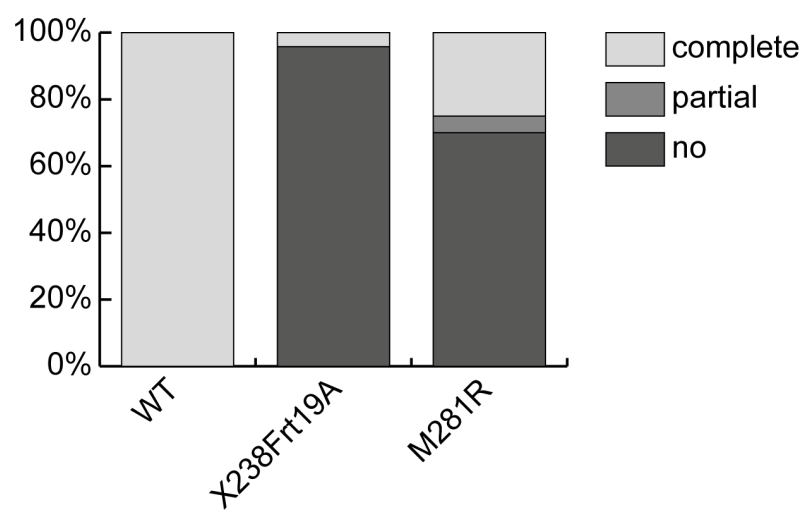

Figure 21: The shmt mutant alleles X238 and M281R show a similar blastoderm phenotype. WT, X238, and M281R embryos were recorded by DIC imaging and their number of syncytial cell cycles (A) and their cellularisation progress (B) was determined from the timelapse recordings. (A) The syncytial cell cycle program of WT embryos underwent 14 cycles. The majority of both X238 and M281R embryos stopped one cycle earlier $(83.3 \%$ and $66.7 \%$, respectively). A minor part of the X238 and M281R embryos performed mitosis 12 or 13 only in a part of the embryo leading to patched situation of 12 / 13 or 13 / 14 syncytial cycles, respectively. Although a higher percentage of M281R embryos was able to progress into cycle 14, the phenotype was comparable to X238. (B) Cellularisation progress was set as "complete", when the cellularisation furrow invaginated below the basal tip of the nuclei and the germband extension initiated as in WT. Cellularisation was set to "no", if no invagination of the furrow was detectable until the majority of nuclei had fallen into the interior of the embryo. If the cellularisation furrow invaginated partially, either in the whole or in a part of the embryo, before the embryo morphology became disturbed, the cellularisation progress was set as "partial". Both X238 and M281R embryos were not able to cellularise (95.8\% and 70\%, respectively). Similar to the cell cycle phenotype, a higher percentage of M281R embryos finished cellularisation compared to X238, but the cellularisation phenotype was similar to X238. The bar charts illustrate the results presented in Tables 3 and 4 . 
Table 3: The mutant alleles X238 and M281R display a similar cell cycle phenotype.

\begin{tabular}{lccccr}
\hline & \multicolumn{4}{c}{ Syncytial cycles (\%) } \\
\cline { 2 - 5 } Genotype & $\mathbf{1 2} \backslash \mathbf{1 3}$ & $\mathbf{1 3}$ & $\mathbf{1 3} \backslash \mathbf{1 4}$ & $\mathbf{1 4}$ & $\mathbf{n}$ \\
\hline WT & 0 & 0 & 0 & 100 & 7 \\
X238Frt19A & 4.2 & 83.3 & 4.2 & 8.3 & 24 \\
M281R & 0 & 66.7 & 9.5 & 23.8 & 21 \\
\hline
\end{tabular}

Table 4: The cell cycle phenotype of M281R is similar to that of X238.

\begin{tabular}{lcccr}
\hline & \multicolumn{3}{c}{ Cellularisation (\%) } & \\
\cline { 2 - 4 } Genotype & no & partial & complete & n \\
\hline WT & 0 & 0 & 100 & 7 \\
X238Frt19A & 95.8 & 0 & 4.2 & 24 \\
M281R & 70 & 5 & 25 & 20 \\
\hline
\end{tabular}

\subsubsection{The penetrance of the $X 238$ phenotype depends on the age of the mother}

Observing X238 germline clones throughout a period of several weeks indicated that the older the mother flies became, the penetrance of the phenotype decreased. Systematic analysis by time-lapse imaging of embryos laid by flies of a defined age confirmed this hypothesis (Fig. 22). 84.2\% of the embryos from $10 \mathrm{~d}$ old mother flies stopped the cell cycle progression in cycle 13 (Figure 22A, , Table 5). This percentage decreased to $20 \%$ and $40 \%$ in embryos from $18 \mathrm{~d}$ and $24 \mathrm{~d}$ old mother flies, respectively. Whereas $5.3 \%$ of the embryos from $10 \mathrm{~d}$ old mother flies developed into cycle 14, $55 \%$ and $50 \%$ of the embryos from $18 \mathrm{~d}$ and $24 \mathrm{~d}$ old mother flies were able to finish mitosis 13 . Also the proportion of cellularising embryos increased upon ageing of the mother flies. $5.3 \%$ of the embryos from young flies $(10 \mathrm{~d})$ compared to $71.4 \%$ and $66.7 \%$ of embryos laid by older flies (18d and $24 \mathrm{~d}$, respectively) were able to finish cellularisation (Figure 22B, Table 6). Independent of the mother's age, the development of the embryos stopped latest during germband extension (data not shown).

\subsubsection{The $\mathrm{X} 238$ phenotype is not dependent on the temperature}

After finding an age-dependency in phenotype formation, we asked if the temperature is involved in phenotype development. Germline clones were generated as described, but growing of the flies after heat shock was carried out at the desired temperatures 
A

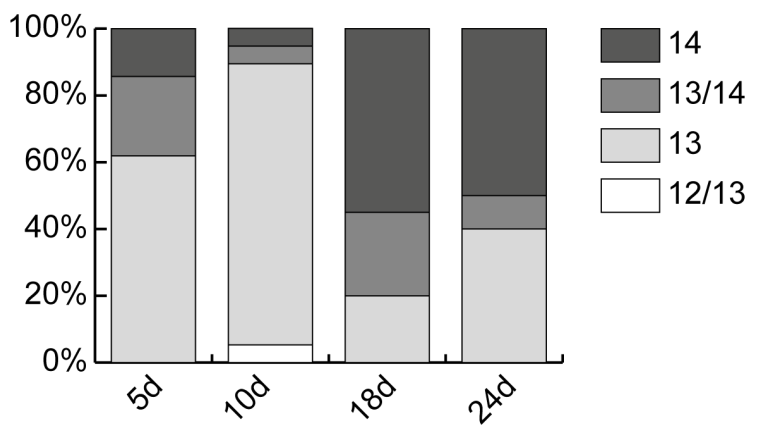

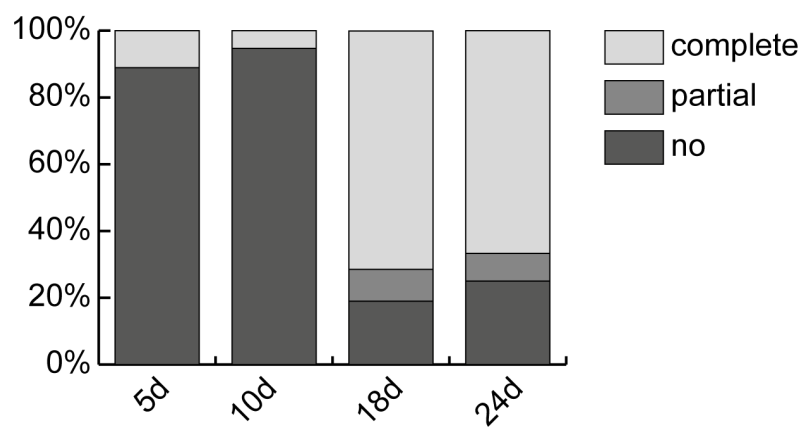

Figure 22: The blastoderm phenotype depends on the age of the mother flies. X238 embryos laid by mother flies of defined age were recorded by DIC imaging. The number of syncytial cell cycles (A) and their cellularisation progress (B) was determined from the timelapse recordings as described in Figure 21. (A) With increasing age of the mother flies, a higher percentage of the embryos underwent 14 cycles (50\% from 24 days compared to $5.3 \%$ of 10 days old mother flies). (B) Similar to this, also the ability to cellularise increased with increasing age of the mother flies (66.7\% from 24 day compared to $5.3 \%$ of 10 days old mother flies). The bar charts visualise the data presented in Tables 5 and 6 .

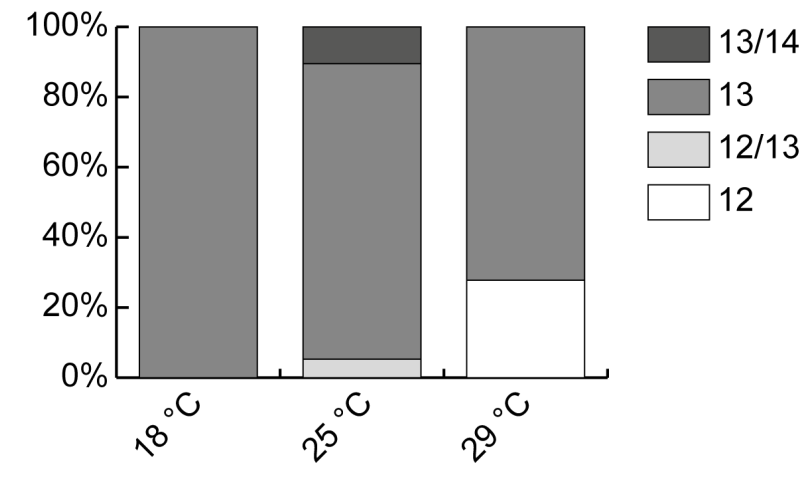

Figure 23: The X238 phenotype is not sensitive to the temperature. X238 embryos from germ line clone (glc) crosses kept at defined temperatures were recorded by DIC imaging. The number of syncytial cell cycles was determined from the time-lapse recordings as described in Figure 21. Although a minor part of the analysed embryos of the glc cross kept at $29^{\circ} \mathrm{C}$ stopped their cell cycle progression in cycle 12 , the temperature had no significant influence on the phenotype of X238. The bar chart represents the results from Table 7 . 
Table 5: The cell cycle phenotype of X238 mutant embryos is age-dependent.

\begin{tabular}{lccccc}
\hline & \multicolumn{4}{c}{ Syncytial cycles (\%) } & \\
\cline { 2 - 5 } Age & $\mathbf{1 2} \backslash \mathbf{1 3}$ & $\mathbf{1 3}$ & $\mathbf{1 3} \backslash \mathbf{1 4}$ & $\mathbf{1 4}$ & $\mathbf{n}$ \\
\hline $5 \mathrm{~d}$ & 0 & 61.9 & 23.8 & 14.3 & 21 \\
$10 \mathrm{~d}$ & 5.3 & 84.2 & 5.3 & 5.3 & 19 \\
$18 \mathrm{~d}$ & 0 & 20 & 25 & 55 & 20 \\
$24 \mathrm{~d}$ & 0 & 40 & 10 & 50 & 10 \\
\hline
\end{tabular}

Table 6: The ability of X238 mutant embryos to cellularise is dependent on the age of the mothers.

\begin{tabular}{llcrr}
\hline & \multicolumn{3}{c}{ Cellularisation (\%) } \\
\cline { 2 - 4 } Genotype & no & partial & complete & n \\
\hline $5 \mathrm{~d}$ & 88.9 & 0 & 11.1 & 18 \\
$10 \mathrm{~d}$ & 94.7 & 0 & 5.3 & 19 \\
$18 \mathrm{~d}$ & 19 & 9.5 & 71.4 & 21 \\
$24 \mathrm{~d}$ & 25 & 8.3 & 66.7 & 12 \\
\hline
\end{tabular}

(Fig. 23). Lowering the temperature to $18^{\circ} \mathrm{C}$ or increasing to $29^{\circ} \mathrm{C}$ had no significant effect on the number of cell cycles passed by the mutant. At $18^{\circ} \mathrm{C}$ all analysed embryos performed 12 mitoses. This rate was lowered to $84.2 \%$ and $72.2 \%$, when the germline clones were raised at $25^{\circ} \mathrm{C}$ and $29^{\circ} \mathrm{C}$, respectively (Table 7 ).

Table 7: Temperature does not influence the manifestation of the phenotype.

\begin{tabular}{lccccr}
\hline & \multicolumn{5}{c}{ Syncytial cycles (\%) } \\
\cline { 2 - 5 } Temperature & $\mathbf{1 2}$ & $\mathbf{1 2} \backslash \mathbf{1 3}$ & $\mathbf{1 3}$ & $\mathbf{1 3} \backslash \mathbf{1 4}$ & $\mathbf{n}$ \\
\hline $18^{\circ} \mathrm{C}$ & 0 & 0 & 100 & 0 & 9 \\
$25^{\circ} \mathrm{C}$ & 0 & 5.3 & 84.2 & 10.5 & 19 \\
$29^{\circ} \mathrm{C}$ & 27.8 & 0 & 72.2 & 0 & 18 \\
\hline
\end{tabular}




\subsection{Enzymatic function of SHMT is necessary for progression into cycle 14 and cellularisation}

The structure of SHMT is well conserved. The only known function is the enzymatic activity in C1-metabolism by catalysing the transition of serine and THF to glycine and 5,10-mTHF. Other functional domains are unknown so far. To address this, alternative rescue constructs were generated (Fig. 24B). We inserted a point mutation in the shmt gene, which abolished the enzymatic function without affecting the overall structure, as predicted by the structure prediction software HHpred. Szebenyi et al. analysed the cleavage mechanism of rabbit cytosolic SHMT (rcSHMT) and generated point mutations of Glu75 to leucine and glutamine. They could show that these mutations did not significantly change the structure of SHMT, but interfered with the folate-dependent serine-to-glycine reaction as well as with the conversion of methenyltetrahydrofolate to 5-formyltetrahydrofolate [152]. For structure prediction the peptide SHMT-PA was aligned against the E75L mutant form of rabbit cytosolic SHMT (Fig. 25). SHMT-PA and rcSHMT-E75L were highly similar in their structure, the majority of the protein sequences overlapped completely (Fig. 25A). Nevertheless, the first 60 aa of SHMT-PA did not align with the structure of rcSHMT, supporting the hypothesis that these amino acids contain the putative mitochondrial localisation signal. Moreover, the amino acids E75L and E130, which are the Drosophila homologue of rabbit E75, were located at exactly the same position facing their residues into the same direction. From the information provided by structural analysis we concluded that mutation of Glu130 into glutamine would disturb enzymatic protein function without changing the structure. The E130Q mutant enzyme was not able to rescue the lethality of X238 mutants. However, homozygous M281R mutant flies were obtained after crossing to SHMT-E130Q indicating an intergenic complementation (Table 8). Similar data were gained for analysing the rescue ability of the cell cycle and cellularisation phenotype (Tables 9 and 10, Fig. 26).

To rule out other functional domains of SHMT beside the enzymatic contribution we replaced the Drosophila protein by the E. coli sequence, which should provide an enzymatically functional SHMT protein with a different quaternary structure. Currently crystal structures of SHMT from mammalian, bacterial, and archaea species are published [21]. The monomeric SHMT structure is highly conserved. But it can be assumed that procaryotic species form an SHMT dimer, whereas the eucaryotic enzymes act as tetramers [23]. Two alternate rescue constructs were cloned, one (Bact) replacing the whole open reading frame (ORF) of Drosophila shmt gene by glyA, the corresponding $E$. coli gene. The second construct (Bact2) contains glyA starting at the second start codon, preserving the 5'UTRs of all three peptides and the first 60 aa of peptide SHMT-PA. The 3'UTR of CG3011 was maintained in both constructs. The construct Bact was not able to rescue the blastoderm defects of shmt mutants, thereby it did not restore 


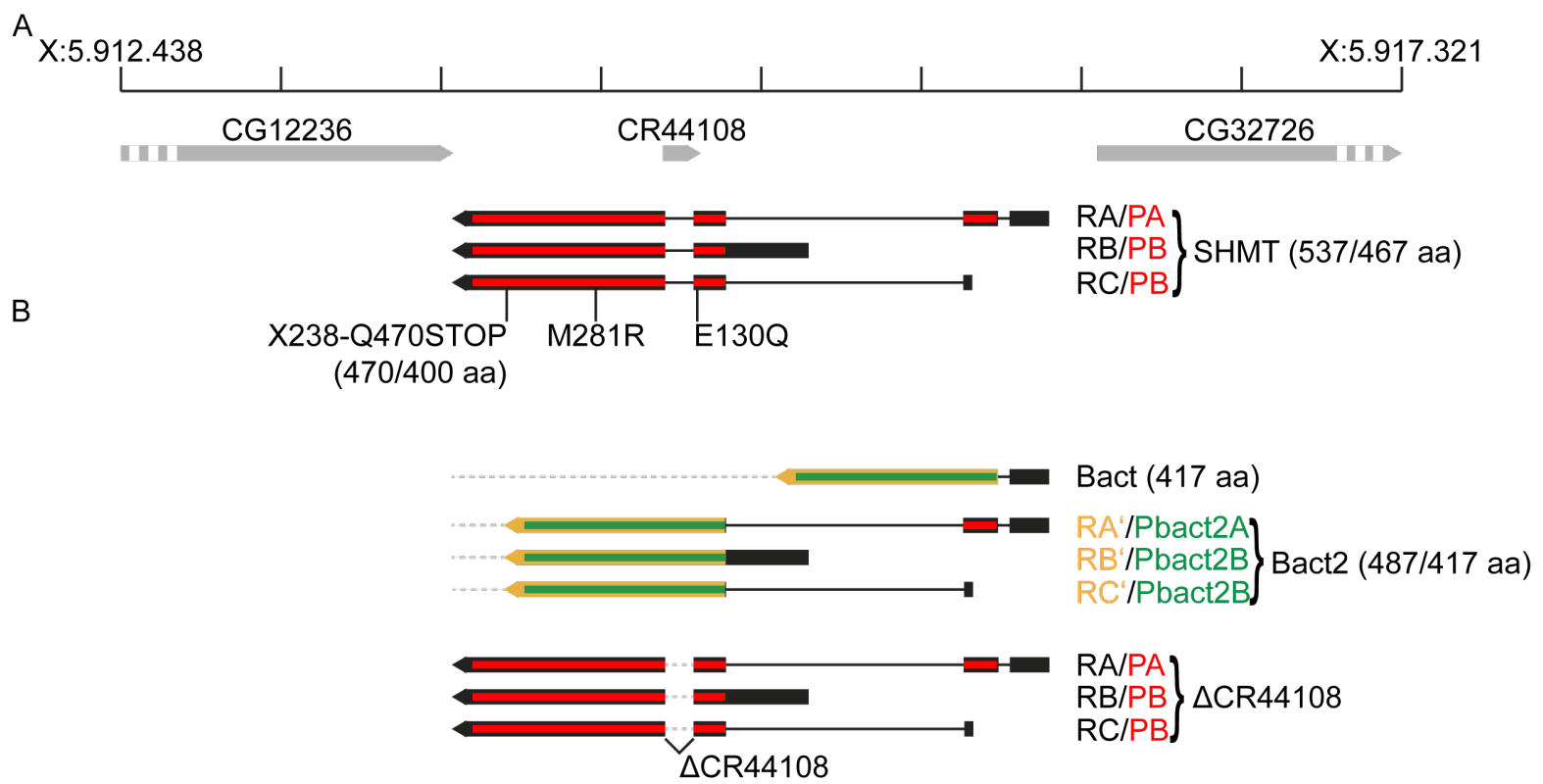

Figure 24: Scheme of the genomic region surrounding the shmt gene indicating transcripts, mutations and rescue constructs. (A) Genomic region X:5.912.438 to $\mathrm{X}: 5.917 .321$, which was used for cloning the rescue construct with WT shmt, is indicated. In black / red the shmt gene is shown, with its three transcripts (RA, RB, and RC) in black boxes, introns in black lines, and the peptides (PA and PB) in red boxes. Neighbouring genes are marked in grey. CG12236 and CG32726 locate partially in this region. (B) Mutant alleles (X238, M281R, E130Q), and rescue constructs are shown including all transcripts and peptides. The whole region depicted in (A) was cloned for each rescue construct, indicated are only changes from WT. WT sequences are depicted in black and red, E. coli sequences, named as Bact and Bact2, in orange (transcript) and green (peptide). Reductions in size are marked in dashed lines.

A

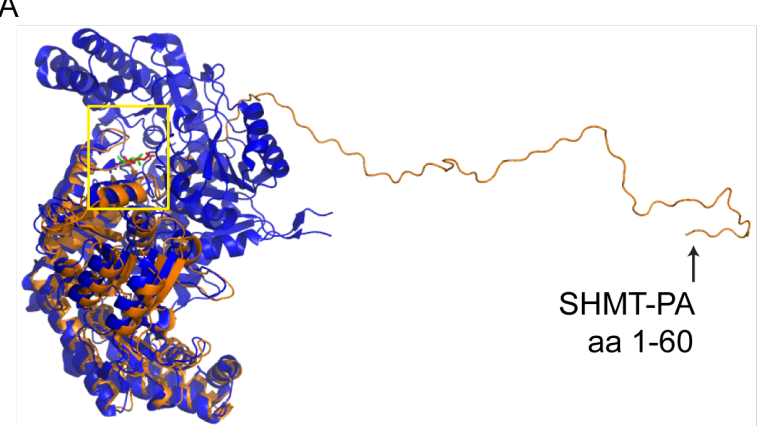

B

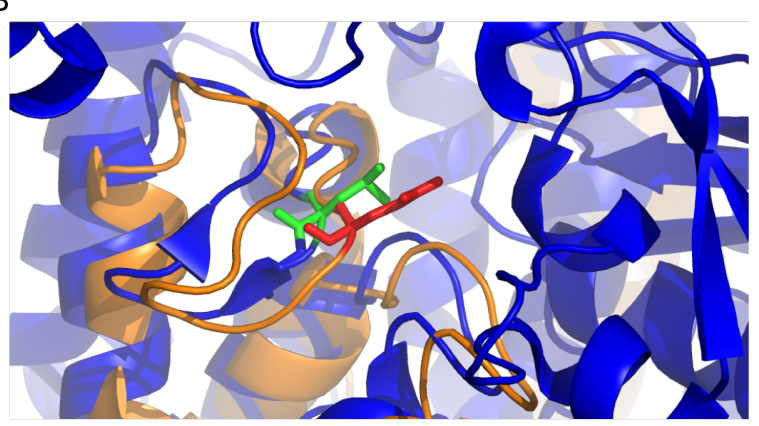

Figure 25: Prediction of Drosophila SHMT structure aligned with rabbit cytosolic SHMT. (A) Drosophila SHMT peptide PA (orange) (see Figure 24A) was aligned against a monomer of rabbit cytosolic SHMT (blue). Both peptides exhibited a high degree of structural overlap. The first 60 aa of Drosophila SHMT did not show a structural homology to rabbit cytosolic SHMT. These aa, encoded in the first exon of the long isoform SHMT-PA could harbour the mitochondrial import sequence. (B) Magnification of the region marked by square box. Localisation of the residues E75L of rcSHMT (green) and E130 of SHMT-PA (red) are highlighted. Both amino acids had a similar position within the structure facing their residues to the same direction. 
Table 8: In trans complementation of the lethal phenotype by the alleles M281R und E130Q.

Number of progeny

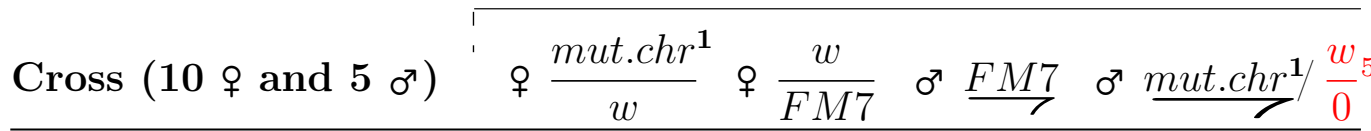

\begin{tabular}{|c|c|c|c|c|c|c|}
\hline \multirow{5}{*}{ 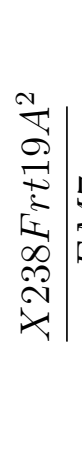 } & \multirow{5}{*}{$\sum_{I_{1}}^{N}$} & $\underline{w} ;$ Rescue & 126 & 118 & 90 & 117 \\
\hline & & $\underline{w} ;$ Bact & 50 & 32 & 34 & 8 \\
\hline & & $\underline{w} ;$ Bact 2 & 202 & 158 & 103 & 125 \\
\hline & & $\underline{w} ; E 130 Q$ & 62 & 51 & 42 & 1 \\
\hline & & $\underline{w} ; \Delta C R 44108$ & 176 & 202 & 104 & 2 \\
\hline \multirow{3}{*}{ 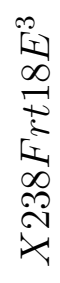 } & \multirow{3}{*}{$\sum_{E}^{N} x$} & $\underline{w} ;$ Rescue & 196 & 177 & 68 & 146 \\
\hline & & $\underline{w} ;$ Bact & 188 & 173 & 85 & 7 \\
\hline & & $\underline{w} ; E 130 Q$ & 281 & 268 & 105 & 7 \\
\hline \multirow{3}{*}{ 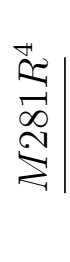 } & & $\underline{w} ;$ Rescue & 353 & 338 & 212 & 315 \\
\hline & $\sum_{E} x$ & $\underline{w} ;$ Bact & 303 & 307 & 172 & 23 \\
\hline & & $\underline{w} ; E 130 Q$ & 378 & 334 & 191 & 280 \\
\hline
\end{tabular}

${ }^{1}$ mutant chromosome: X238Frt19A, X238Frt18E, or M281R

2 X238Frt19A markers: $\mathrm{y}^{-} \mathrm{pn}^{-} \mathrm{sn}^{-}$

3 X238Frt18E markers: $\mathrm{w}^{-} \mathrm{f}^{-} \mathrm{ry}^{+}$

$4 M 281 R$ markers: $\mathrm{y}^{1} \mathrm{w}^{*} \mathrm{ry}^{+\mathrm{t} 7.2}$

5 Males were resulting from non-disjunction event. They did not have markers of mutant chromosomes nor of the balancers. Males were sterile. 
viability (Tables 8, 9, and 10, Fig. 26). However, Bact2 rescued the lethality of shmt mutants (Table 8). This implicated that SHMT does not carry functional domains besides the known enzymatic activity, that are necessary for progression into cycle 14 and cellularisation.

The full genomic rescue could not rule out the possibility that the X238 mutation may affect the expression of a non-coding RNA encoded in the gene $\Delta C R 44108$. So, a construct replacing the shmt gene from the second start codon by the corresponding coding sequence (CDS) was cloned. Thereby most of the $\Delta C R 44108$ gene was deleted. However, introducing this construct did not restore viability indicating either an essential function during Drosophila development or an interference with SHMT expression due to the deleted intron (Table 8).

A

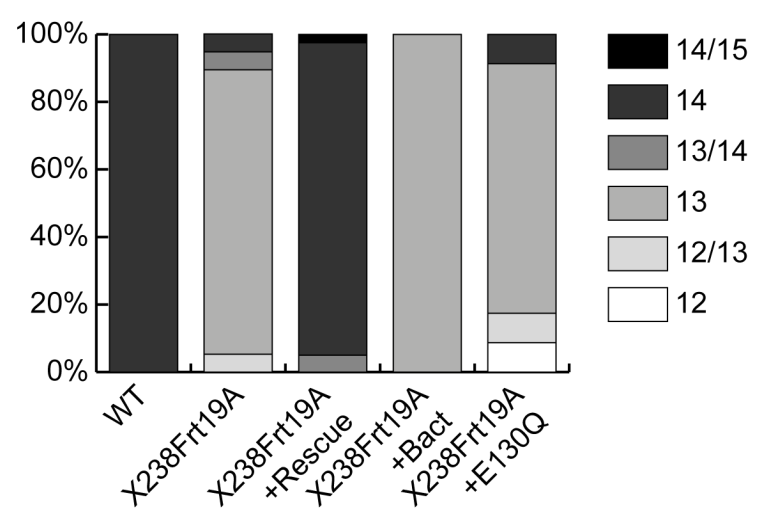

B

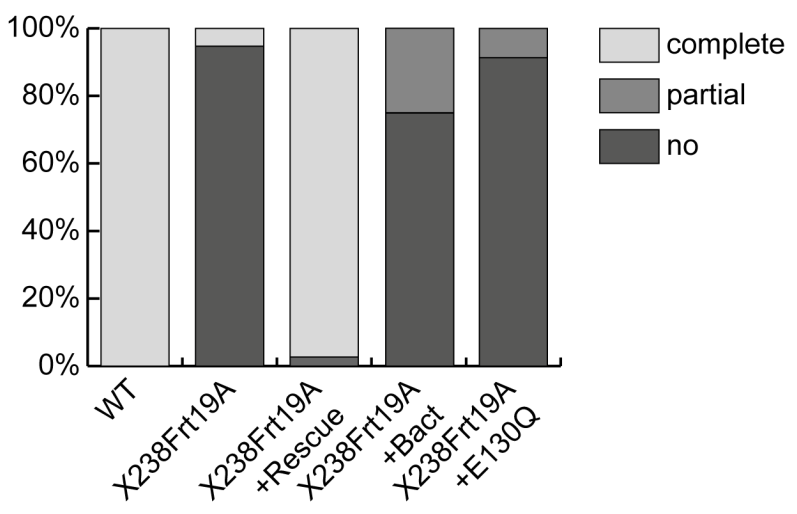

Figure 26: Enzymatic function of SHMT is necessary for cell cycle progression and cellularisation. WT, X238 and X238 embryos harbouring rescue constructs were recorded by DIC imaging and their syncytial cell cycle number (A) and cellularisation ability (B) was determined from the time-lapse recordings as described in Figure 21. (A) $84.2 \%$ of the X238 embryos stopped their syncytial development in cycle 13, one cycle earlier than WT. Introducing the full rescue construct into the mutant background restored the number of syncytial cell cycles, $92.5 \%$ developed to cycle 14. However, expressing the alternative rescue constructs Bact or E130Q in X238 background did not influence the cell cycle phenotype significantly. (B) The majority of the X238 embryos did not cellularise (94.7\%). Expression of the full genomic rescue construct restored the cellularisation ability to $97.3 \%$. Expressing the E. coli SHMT did partially restore the cellularisation phenotype. $25 \%$ of the embryos showed a partial cellularisation. Notwithstanding this, expression of catalytically inactive SHMT was not able to rescue the cellularisation phenotype of X238 significantly. The bar charts represent the results of the rescue experiments from Tables 9 and 10 . 
Table 9: Progression into cycle 14 requires enzymatic function.

\begin{tabular}{lccccccr}
\hline & \multicolumn{7}{c}{ Syncytial cycles (\%) } \\
\cline { 2 - 7 } Genotype & $\mathbf{1 2}$ & $\mathbf{1 2} \backslash \mathbf{1 3}$ & $\mathbf{1 3}$ & $\mathbf{1 3} \backslash \mathbf{1 4}$ & $\mathbf{1 4}$ & $\mathbf{1 4} \backslash \mathbf{1 5}$ & $\mathbf{n}$ \\
\hline WT & 0 & 0 & 0 & 0 & 100 & 0 & 7 \\
X238Frt19A & 0 & 5.3 & 84.2 & 5.3 & 5.3 & 0 & 19 \\
X238Frt19A + Rescue & 0 & 0 & 0 & 5 & 92.5 & 2.5 & 40 \\
X238Frt19A + Bact & 0 & 0 & 100 & 0 & 0 & 0 & 8 \\
X238Frt19A + E130Q & 8.7 & 8.7 & 73.9 & 0 & 8.7 & 0 & 23 \\
\hline
\end{tabular}

Table 10: Cellularisation depends on enzymatical activity.

\begin{tabular}{lcccr}
\hline & \multicolumn{3}{c}{ Cellularisation (\%) } & \\
\cline { 2 - 4 } Genotype & no & partial & complete & n \\
\hline WT & 0 & 0 & 100 & 7 \\
X238Frt19A & 94.7 & 0 & 5.3 & 19 \\
X238Frt19A + Rescue & 0 & 2.7 & 97.3 & 37 \\
X238Frt19A + Bact & 75 & 25 & 0 & 8 \\
X238Frt19A + E130Q & 91.3 & 8.7 & 0 & 23 \\
\hline
\end{tabular}

\subsection{Impaired DNA replication leads to a cell cycle phenotype in X238}

\subsubsection{Twine is not degraded in $\mathrm{X} 238$}

We wanted to analyse if the premature cell cycle pause could be due to a premature onset of zygotic gene expression. DiTalia et al. revealed that degradation of Twine protein at the beginning of cycle 14 is essential for pausing the cell cycle [42] (Figure 27, left panels). Immunostaining of embryos against Twine showed that the protein was not degraded in X238 (Figure 27, right panels). Even late-phase defect mutants had a nuclear Twine signal, which was comparable to WT embryos in cycle 13. This suggested that the zygotic genome is not activated prematurely. This was further confirmed by immunostaining against Frühstart (Frs) (Figure 28). Frs is a cytosolic protein, which becomes expressed at the beginning of cycle 14 and accumulates during cellularisation 

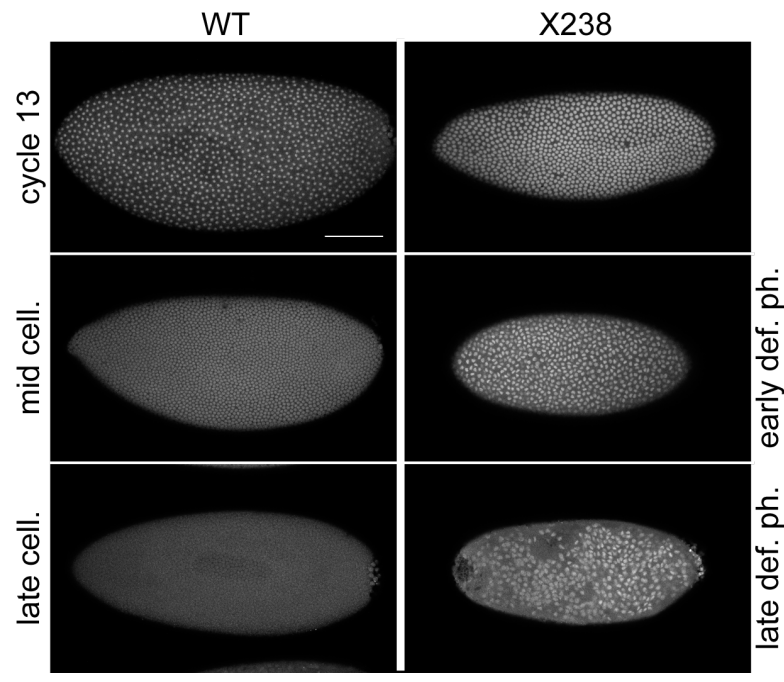

亡ํ Figure 27: The cell cycle pause of X238 does not depend on Twine degradation. Immunostaining of WT and X238 embryos with Twine antibody. In WT embryos Twine protein was degraded during cellularisation, thus preventing progression of the cell cycle. X238 mutants showed a prominent staining of Twine although in late defect phase. Scale bar: $100 \mu \mathrm{m}$.

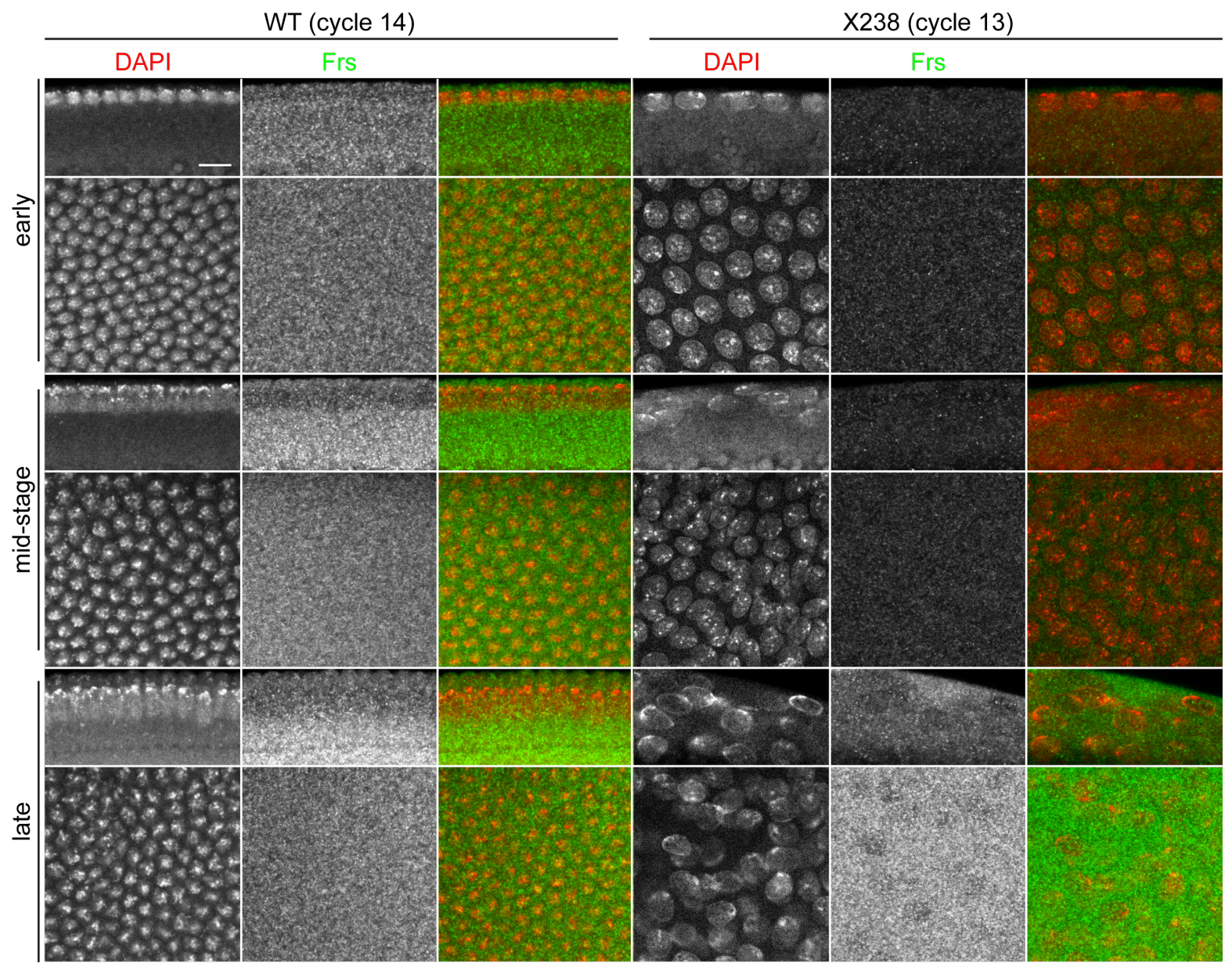

Figure 28: X238 mutant embryos do not express Frs prematurely. WT and X238 embryos were immunostained with Frs antibody and the DNA dye DAPI. The cytosolic protein Frs, which was expressed during cycle 14 in WT, showed a signal in X238 only in the late defect phase. Scale bar: $10 \mu \mathrm{m}$. 
[69]. X238 embryos did not exhibit a significant increase in Frs localisation before late defect phase.

\subsubsection{X238 phenotype depends on zygotic gene expression}

To rule out if the X238 phenotype is dependent on zygotic factors, the transcriptional inhibitor $\alpha$-amanitin was injected in early stage embryos expressing HisRFP and their further development was observed (Fig. 29). WT embryos blocked in transcription were not able to pause the cell cycle and continued cycling rapidly with exhibiting increasing mitotic defects. $92.3 \%$ of the injected embryos entered mitosis $14,46.2 \%$ of them started mitosis 15 (Table 11). Injection of $\alpha$-amanitin into X238 embryos had a similar effect (Tables 11 and 12). $81.8 \%$ of the embryos went into mitosis 13, $9.1 \%$ of them initiated mitosis 14. Embryos entering mitosis 13 either showed a mitotic catastrophe phenotype or were able to enter the next interphase and stopped in the following mitosis. If they entered the next interphase, they showed severe nuclear defects. Sometimes the cell cycle continued also only in one part of the embryo. These results indicated that the manifestation of the X238 cell cycle phenotype depends on zygotic gene expression.

Table 11: X238 mutants blocked in transcription enter mitosis 13.

\begin{tabular}{lcccccr}
\hline & \multicolumn{5}{c}{ Syncytial mitoses (\%) } \\
\cline { 2 - 6 } Genotype & $\mathbf{1 2}$ & $\mathbf{1 2} \backslash \mathbf{1 3}$ & $\mathbf{1 3}$ & $\mathbf{1 3} \backslash \mathbf{1 4}$ & $\mathbf{1 4}$ & $\mathbf{n}$ \\
\hline WT & 0 & 0 & 100 & 0 & 0 & 5 \\
X238Frt19A & 87.5 & 12.5 & 0 & 0 & 0 & 16 \\
\hline WT $+\alpha$-aman. & 0 & 0 & 7.7 & 38.5 & 46.2 & 13 \\
X238Frt19A $+\alpha$-aman. & 18.2 & 45.5 & 27.3 & 9.1 & 0 & 11 \\
\hline
\end{tabular}

Table 12: The X238 phenotype depends on zygotic transcription.

\begin{tabular}{lrrrrrc}
\hline & & \multicolumn{5}{c}{ Number of nuclei in $\mathbf{5 0} \mu \mathrm{m}^{\mathbf{2}}$ in cycle } \\
\cline { 3 - 7 } Genotype & $\mathbf{n}$ & $\mathbf{1 0}$ & $\mathbf{1 1}$ & $\mathbf{1 2}$ & $\mathbf{1 3}$ & $\mathbf{1 4}$ \\
\hline WT & 4 & $5.5 \pm 0.5$ & $9.5 \pm 0.5$ & $19.5 \pm 2.8$ & $39.8 \pm 3.3$ & $74.8 \pm 1.3$ \\
X238Frt19A & 15 & $5.1 \pm 0.9$ & $9.2 \pm 1.6$ & $20.3 \pm 3.1$ & $37.1 \pm 3.6$ & \\
\hline WT $+\alpha$-aman. & 13 & $5.3 \pm 0.7$ & $9.6 \pm 1.4$ & $18.5 \pm 2.5$ & $33.9 \pm 3.8$ & $44.2 \pm 7.2$ \\
X238Frt19A $+\alpha$-aman. & 11 & $5.0 \pm 1.0$ & $10.0 \pm 1.2$ & $20.7 \pm 1.7$ & $36.8 \pm 4.6$ & \\
\hline
\end{tabular}




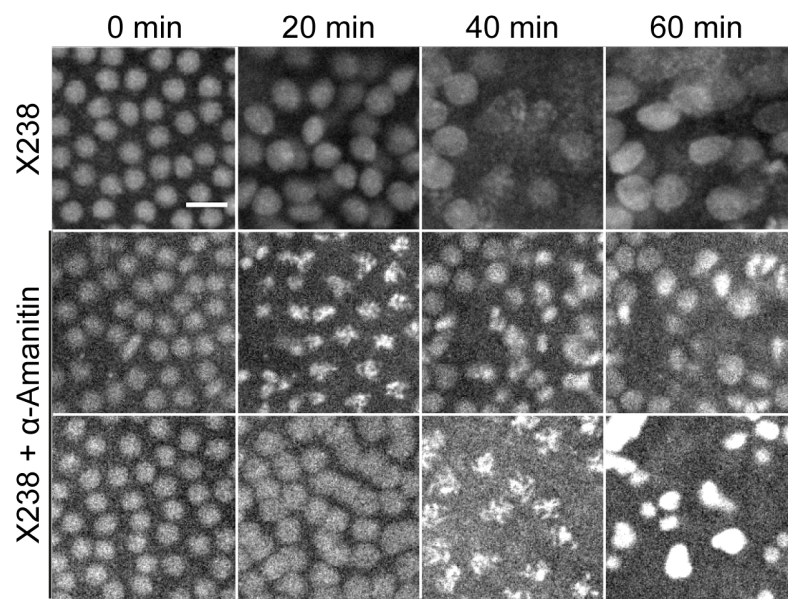

Figure 29: Blocking transcription with $\alpha$-Amanitin in X238 mutants leads to progression into mitosis 13. $\alpha$-Amanitin was injected into early embryos expressing HisRFP and development was followed by time-lapse recordings. Uninjected X238 embryos did not enter mitosis 13 . However, upon injection of $\alpha$-Amanitin the embryos progressed into mitosis 13 with variable degrees of mitotic defects. $t=0$ min indicates start of interphase 13. Scale bar: $10 \mu \mathrm{m}$.

\subsubsection{Checkpoint activation in SHMT mutants}

\section{SHMT mutants exhibit a nuclear retention of specific mRNAs}

A third possibility which could lead to the premature cell cycle stop is the activation of a checkpoint preventing the cell cycle to progress. In 2014, Iampetro et al. published that activation of Checkpoint kinase 2 (Chk2) (Monk in Drosophila) in syncytial embryos leads to nuclear retention of specific mRNAs including $k u k$ mRNA [76]. To clarify if this also happens in X238 mutants, in situ hybridisation against $k u k$ mRNA was performed. First, the specificity of the $k u k$ probe was tested (Fig. 30). Whereas a localised signal of $k u k$ mRNA could be observed in WT and X238 embryos, there was no specific staining detectable in $\Delta$ Kuk embryos, lacking the $k u k$ gene. In WT embryos $k u k$ mRNA was accumulated cytoplasmatically during cellularisation (Fig. 31). Nascent mRNAs were visible as dots localised in the nuclei from cycle 13 onwards. The frequency of nuclei showing these dotted localisation decreased during cellularisation. X238 embryos also showed dotted expression of nascent mRNAs, but accumulated kuk mRNA additionally in nuclei during interphase 13. In late defect phase this nuclear staining diminished. This results indicated that the Chk2-dependent checkpoint might be activated in X238 mutants.

A similar inhomogeneous protein staining pattern as for the Kuk protein was observed for Slam. Not all embryos exhibited Slam staining and if staining was observed, its intensity varied between embryos of the same immunostaining experiment. So we wondered if slam mRNA is also retained and tested its expression by in situ hybridisation. As already published [168], slam mRNA accumulates at the furrow and in basal particles co-localising with Slam protein. In X238 the co-localisation was similar to WT, but slam accumulated additionally in the nuclei confirming the result obtained with kuk in situ hybridisation (Fig. 32). 


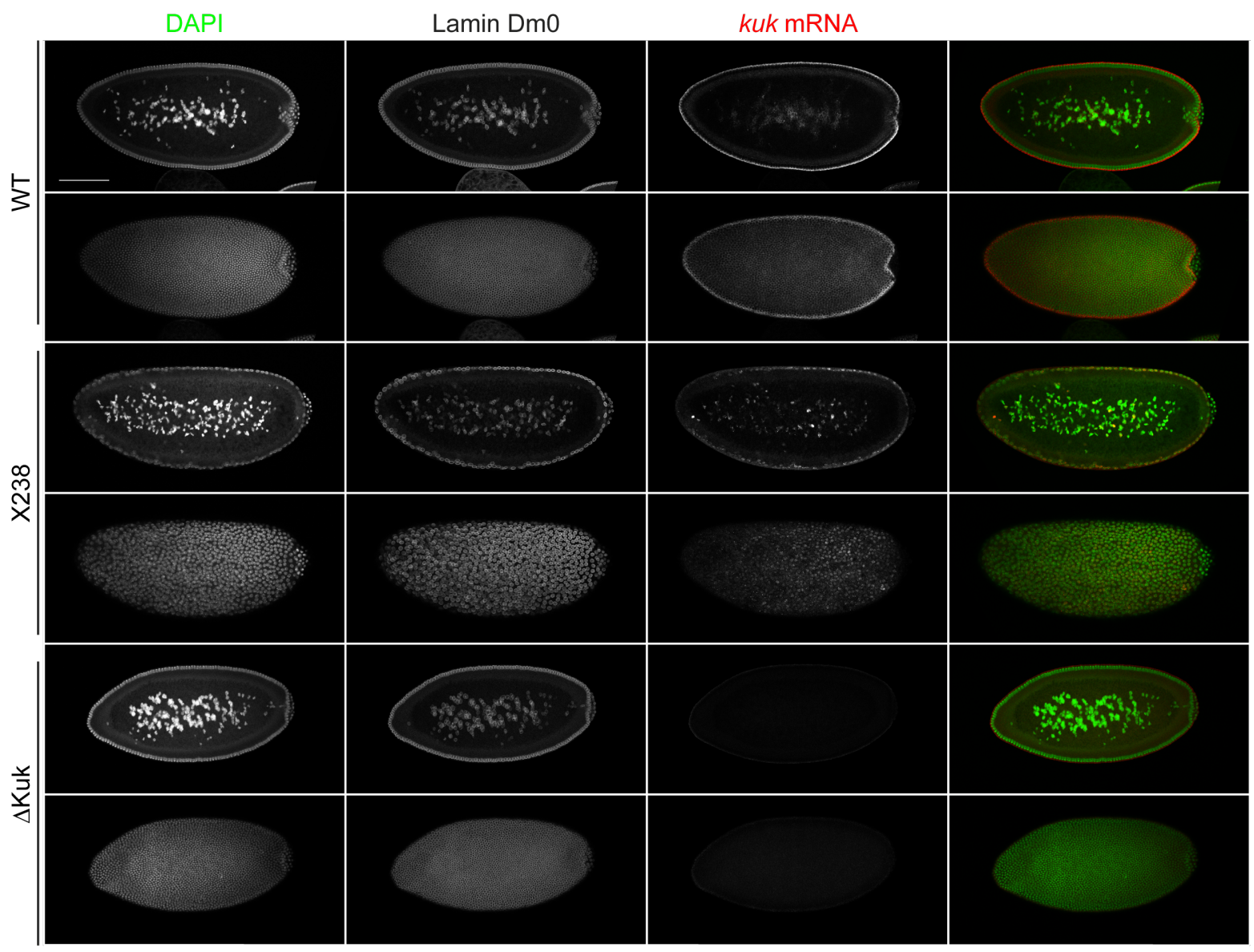

Figure 30: $k u k$ in situ hybridisation is specific for $k u k$ mRNA. Whole mount in situ hybridisation against $k u k$ mRNA and immunostaining with an antibody against Lamin Dm0 to mark the nuclear envelope and DAPI to stain for DNA. In situ hybridisation showed a specific signal in WT and X238 embryos but not in the $k u k$-deficient $\Delta$ Kuk embryos. $100 \mu \mathrm{m}$.

The X238 phenotype partially depends on the checkpoint kinase Grapes

To reveal if Checkpoint kinase 1 (Chk1) (Grapes in Drosophila) mediated checkpoint is active in X238, we injected H1-Alexa488 into grapes mutants and double mutants of X238 and grapes to visualise the nuclei and recorded their development. Grapes mutant embryos fail to stop their cell cycle at cycle 14 and continue to divide by establishing increasing DNA damage. Appr. $60 \%$ of the double mutant embryos entered mitosis 13 , but only $17 \%$ also exhibited the mitotic catastrophe phenotype of grapes mutants. This indicated a partial dependence of the X238 phenotype on the checkpoint function mediated by Grapes. 


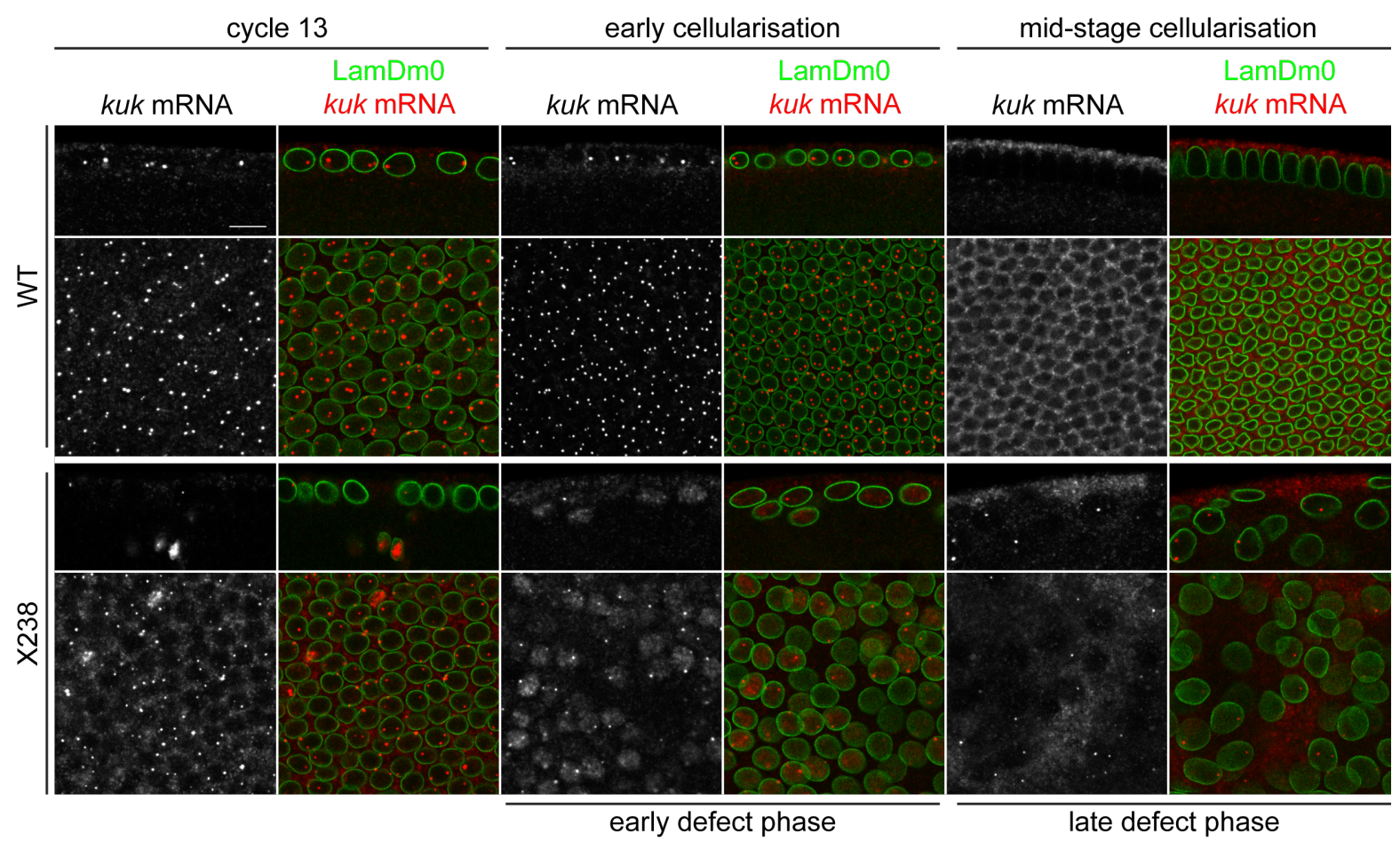

Figure 31: $k u k$ mRNA localisation is altered in X238. Whole mount in situ hybridisation of $k u k$ mRNA in WT and X238 embryos co-immunostained with Lamin Dm0 antibody to visualise the nuclear envelope and DAPI to label the DNA. WT embryos accumulated only primary transcripts of $k u k$ mRNA in the nuclei, whereas mature mRNAs were located in the cytoplasm. In X238 however, mature kuk mRNA was partly localised in nuclei. Scale bar: $10 \mu \mathrm{m}$.

\subsection{4 $\mathrm{S}$ phase progression in shmt mutants}

\section{X238 embryos exhibit a prolonged $S$ phase in cycle 13}

The observation that X238 embryos were able to process into mitosis 13, when DNA replication checkpoint was mutated, led us to the hypothesis that DNA replication is disturbed in cycle 13 of X238 mutant embryos. So we wanted to analyse DNA replication in detail by EdU incorporation. Whereas WT embryos before and early in cellularisation showed a uniform signal in the nuclei due to early replication, which occurs uniformly throughout the nucleus. Embryos later in cellularisation had a restricted staining to the late replicating centromeric heterochromatin in the apical part of the nuclei (Fig. 34 and 36). Embryos in mitosis did not incorporate EdU. Early X238 embryos showed a similar behaviour of EdU incorporation than WT. In cycle 13 the X238 embryos continued to include EdU in the DNA indicating active replicating DNA, even in the late defect phase. Furthermore, embryos in early defect phase displayed a speckled staining for EdU. Although the majority of the nuclei incorporated EdU, some nuclei did not (Fig. 


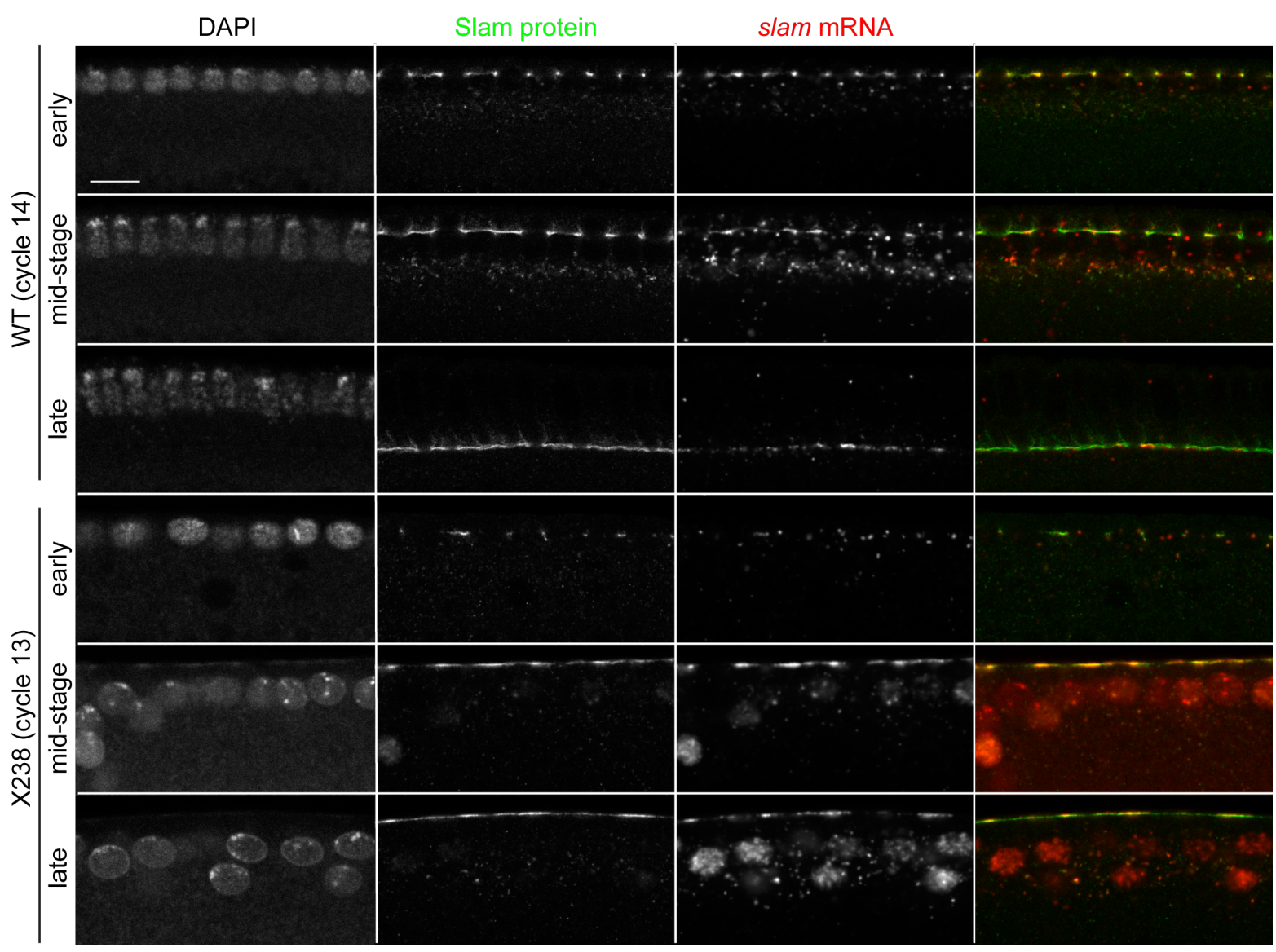

Figure 32: slam mRNA is retained in the nuclei in X238 embryos. Whole mount in situ hybridisation of slam mRNA coupled to immunostaining against Slam protein of WT and X238 embryos. slam mRNA and Slam protein colocalised at the furrow canal and basal particles during cellularisation in WT. However, in X238 embryos slam accumulated additionally in the nuclei. Scale bar: $10 \mu \mathrm{m}$.

35). In Fig. 36 a detailed view of these embryos is shown revealing that some nuclei had a higher signal intensity than others (embryo in the upper right panel). Embryos in defect phase exhibited an enrichment of EdU at the nuclear periphery as well as some signal intense spots within the nuclei. This peripheral staining became more prominent in late defect phase. It was similar to the DNA staining of these embryos implicating that actively replicating DNA accumulates at the nuclear envelope. It has to be noted that EdU incorporation was performed for $15 \mathrm{~min}$ and so the images show the sum of replication during this time.

Continual replication can result in over-replication of chromosomes. To determine if over-replication happens in the mutant, immunostainings against Collision Induced Dissociation (CID), the Drosophila homologue of the CENP-A centromere-specific H3-like proteins, were performed. It is localised at the centromeres of chromosomes [26]. As 


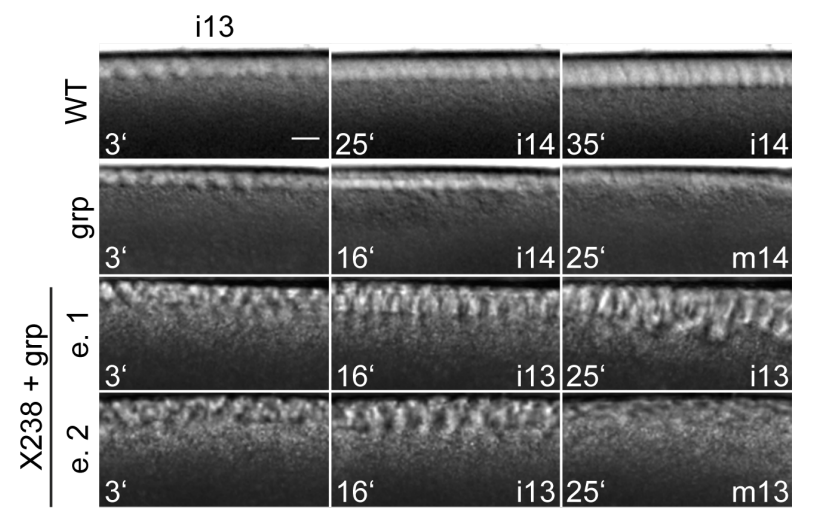

Figure 33: The X238 phenotype is partially dependent on the activation of the Grapes checkpoint. Time-lapse recording of embryos injected with H1-Alexa488. WT embryos paused their cell cycle progression in cycle 14 to perform cellularisation. grapes mutant embryos did not pause in cycle 14, but continued to divide and exhibited a mitotic catastrophe phenotype. X238 mutants stopped their development in interphase 13. However, a fraction of double mutant embryos of X238 and grapes entered mitosis 13 and showed the mitotic catastrophe phenotype of grapes mutants. Scale bar: $10 \mu \mathrm{m}$.

Table 13: Mutation of the checkpoint kinase Grapes promotes entry into mitosis 13 in X238 embryos.

\begin{tabular}{lccr}
\hline & \multicolumn{2}{c}{ Syncytial mitoses (\%) } \\
\cline { 2 - 3 } Genotype & $\mathbf{1 2}$ & $>\mathbf{1 2}$ & $\mathbf{n}$ \\
\hline grp $^{-/-}$ & 0 & 100 & 4 \\
X238Frt18E + grp $^{-/+}$ & 100 & 0 & 4 \\
X238Frt18E $+\mathrm{grp}^{-/-}$ & 41.7 & 58.8 & 12 \\
\hline
\end{tabular}

Table 14: A fraction of X238, Grapes double mutants showed a mitotic catastrophe phenotype similar to grapes mutants.

\begin{tabular}{|c|c|c|c|}
\hline Genotype & X238 phenotype & grapes phenotype & $\mathbf{n}$ \\
\hline $\mathrm{X} 238 \mathrm{Frt18E}+\mathrm{grp}^{-/+}$ & 100 & 0 & 5 \\
\hline $\mathrm{X} 238 \mathrm{Frt} 18 \mathrm{E}+\mathrm{grp}^{-/-}$ & 83.3 & 16.7 & 12 \\
\hline
\end{tabular}




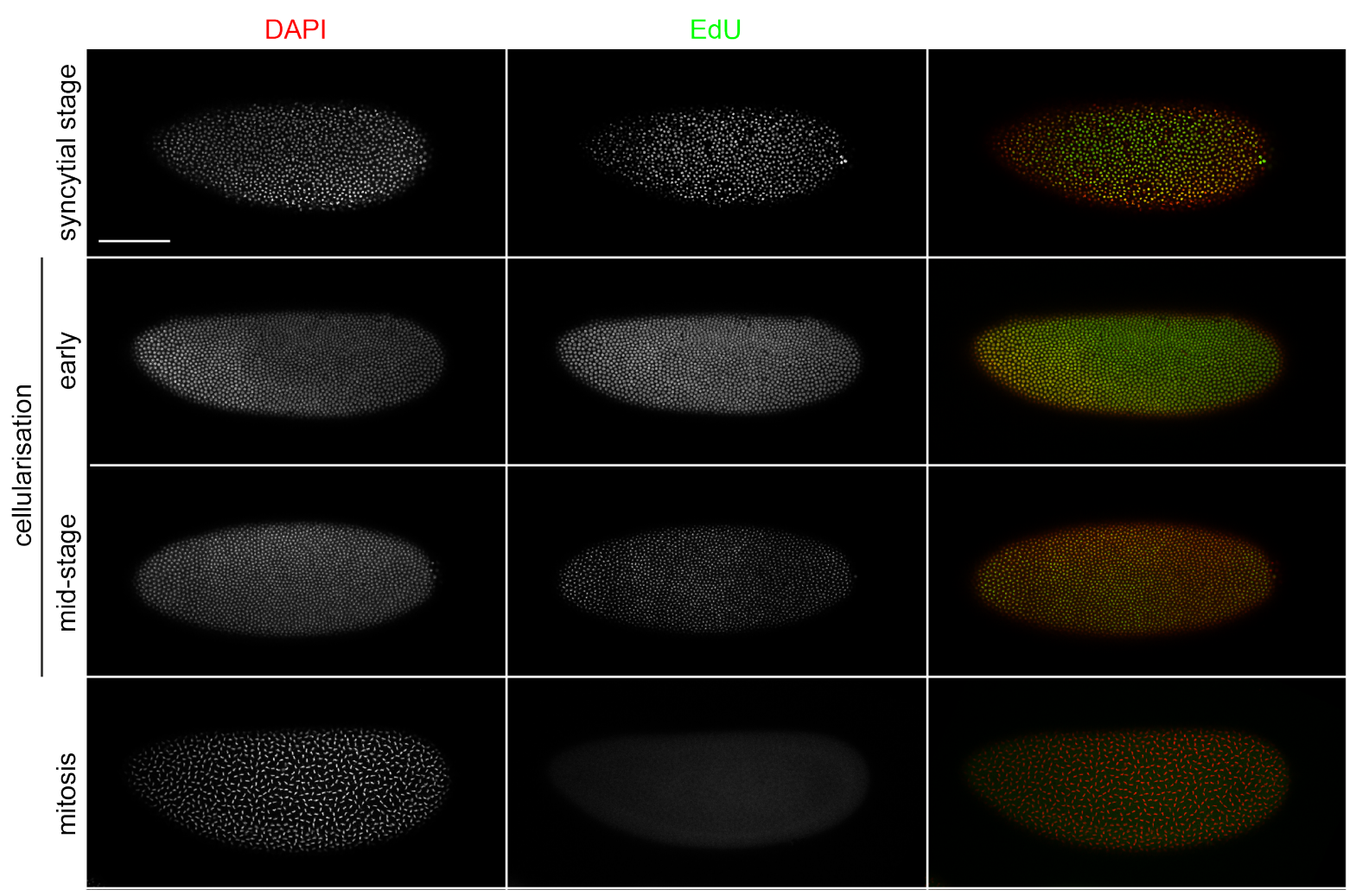

Figure 34: EdU is incorporated ubiquitously in the syncytial blastoderm nuclei of WT embryos. Permeabilised embryos were treated with EdU for $15 \mathrm{~min}$ and fixed. EdU was incorporated into the DNA during replication in S phase. Panels show WT embryos of progressing blastoderm stage, the lower panel depicts an embryo in metaphase, where no EdU staining was visible. Scale bar: $100 \mu \mathrm{m}$. 


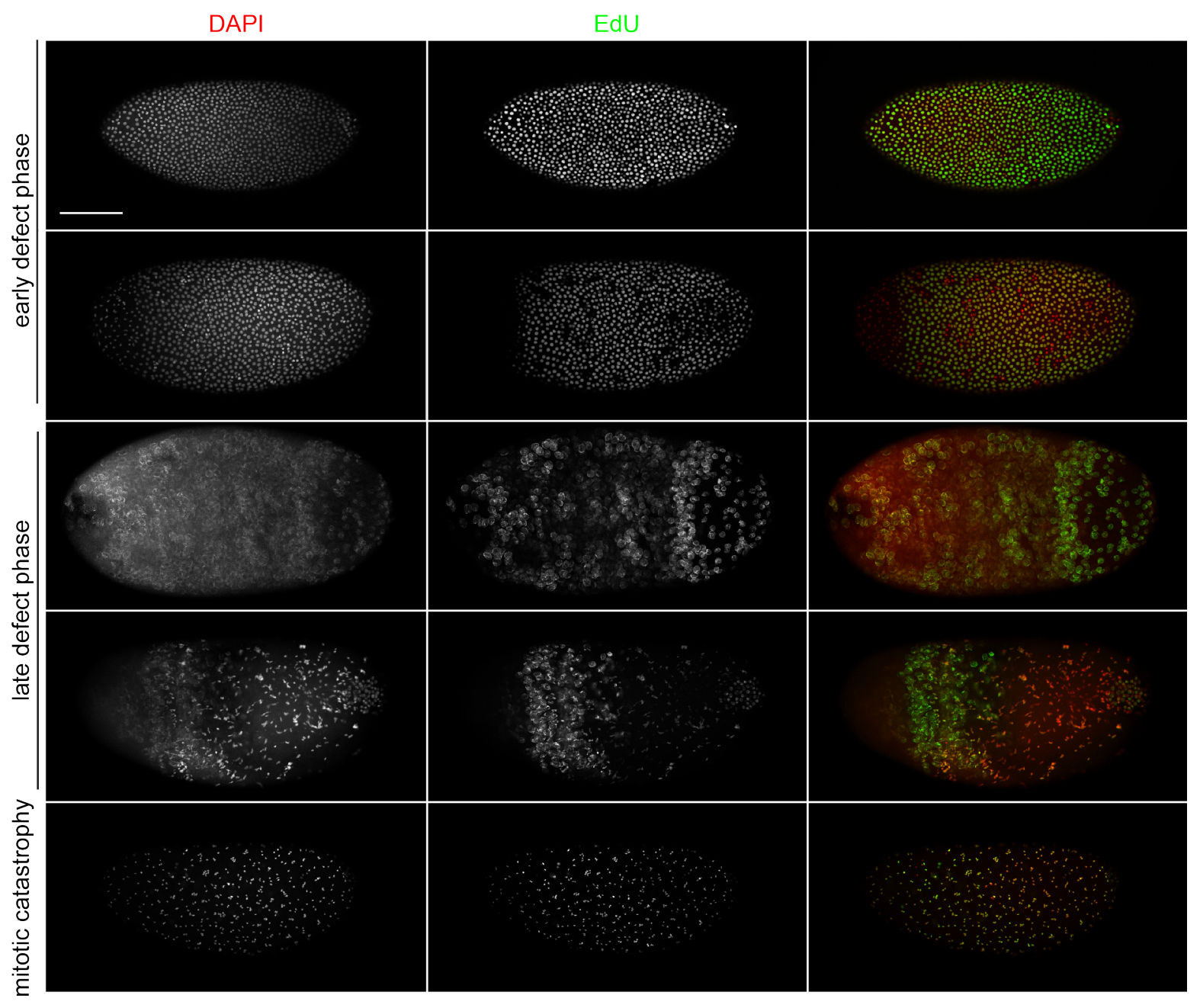

Figure 35: Pattern of EdU incorporation in X238 is irregular and persists until late-phase defect. Treatment of embryos with EdU was carried out as mentioned in Figure 34. X238 embryos showed a speckled EdU staining, where some nuclei did not have a signal. In embryos in late defect phase EdU incorporation was still detectable. Scale bar: $100 \mu \mathrm{m}$. 


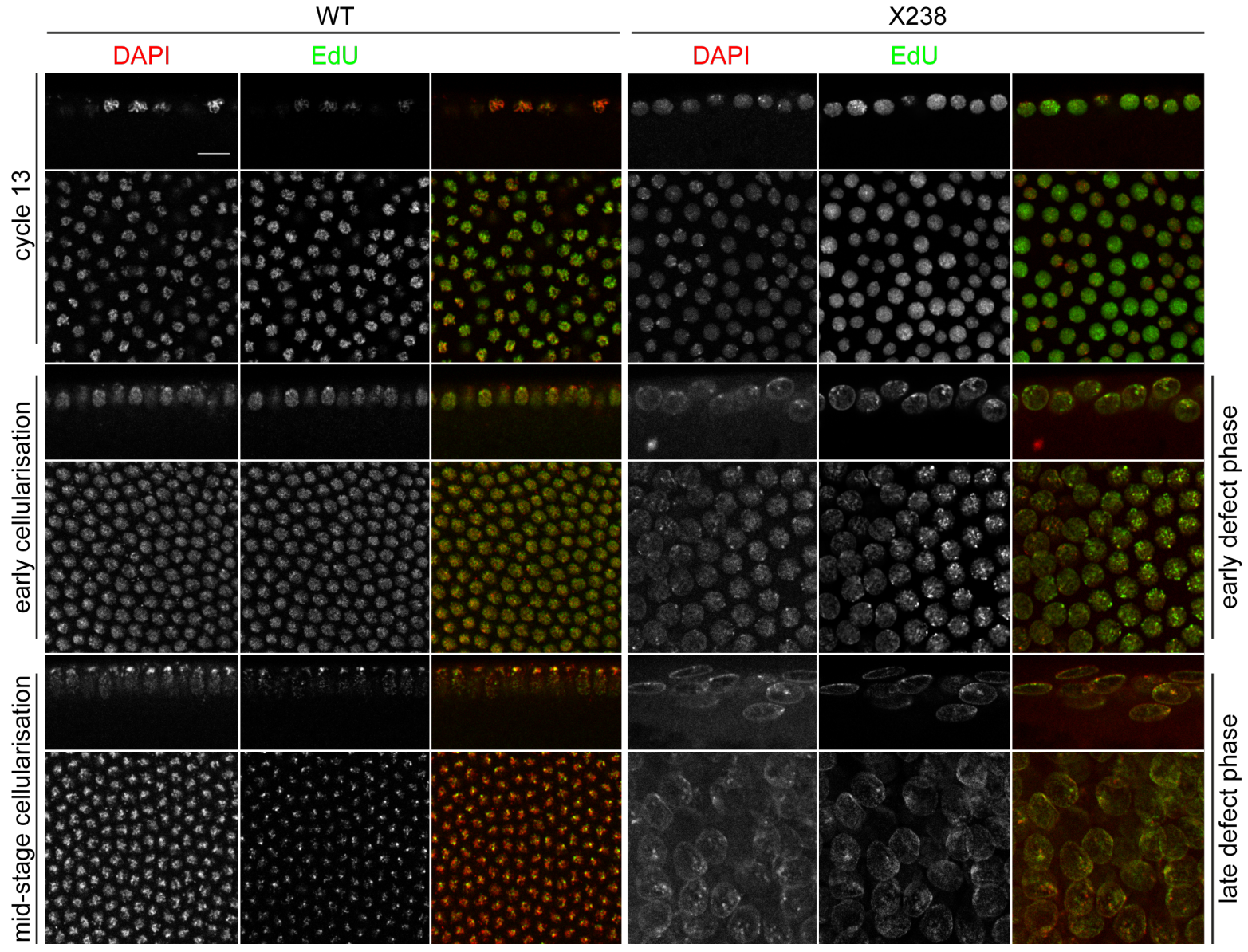

Figure 36: Magnified images of EdU staining. Treatment of embryos with EdU was carried out as mentioned in Figure 34. During WT cellularisation EdU became progressively restricted to the apical heterochromatin of the nuclei. This restriction was not observed in X238 mutants. EdU foci were located throughout the nuclei of X238. In defect phase the signal was enriched at the nuclear periphery. Scale bar: $10 \mu \mathrm{m}$. 


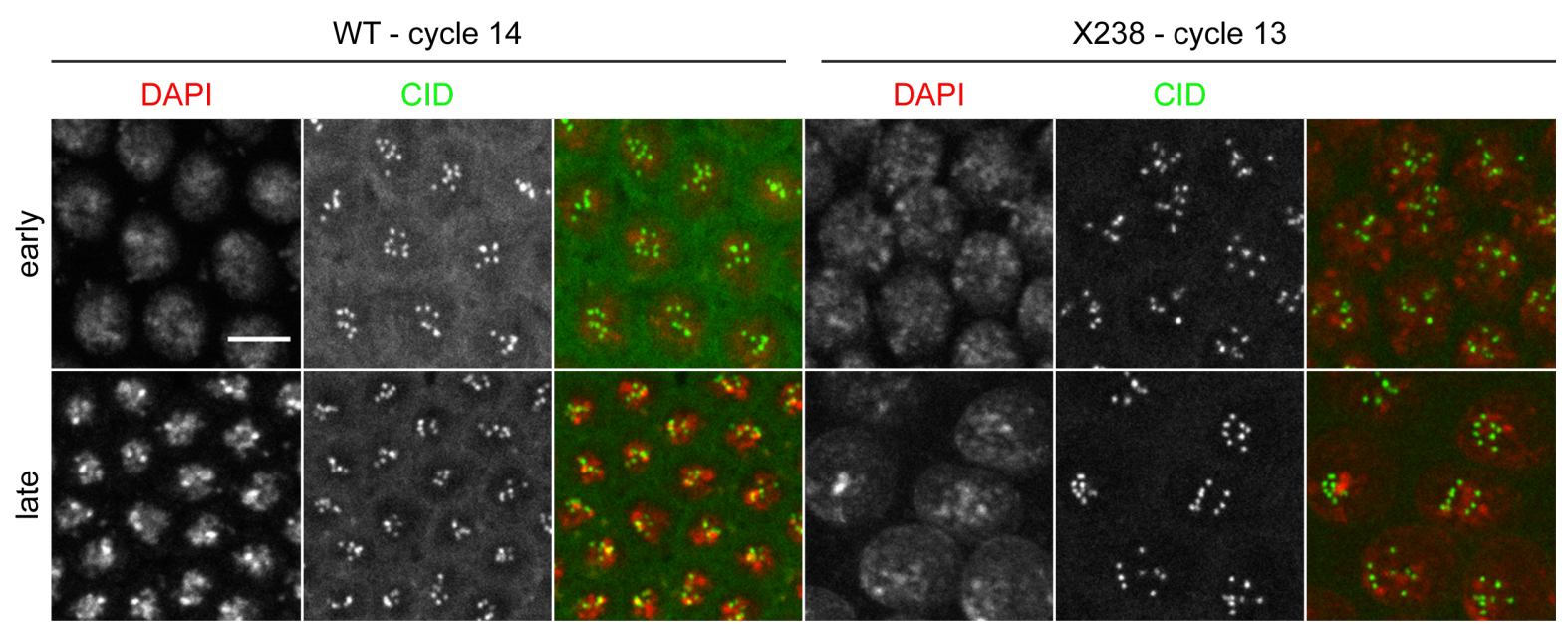

Figure 37: Centromere formation is not affected in X238 mutants. Immunostaining of WT and X238 embryos with CID antibody and the DNA dye DAPI. CID was located at the centromeres of the chromosomes. Drosophila has four chromosomes, so WT each nucleus contained eight spots-like signals of CID protein. A similar CID pattern could be observed in X238 embryos. Also in the late defect phase no overreplication, which would have lead to an increased of CID spots, was detectable. Scale bar: $10 \mu \mathrm{m}$.

Drosophila has four chromosomes with two centromeres each, CID is localised in eight spots in a single nucleus (Fig. 37). CID spot number in X238 nuclei did not differ from WT concluding that X238 did not exhibit over-replication of its DNA.

\section{Temporal resolution of replication by PCNA-GFP}

To further narrow down how exactly the loss of a functional SHMT affects $\mathrm{S}$ phase progression of cycle 13, we performed life imaging. For this approach, we overexpressed GFP-PCNA with the mat-GAL4/UASp system. GFP-PCNA is used as a marker protein for DNA replication [135]. Overexpression of GFP-PCNA does not interfere with cell cycle progression (Tables 15 and 16). Nuclear localisation of GFP-PCNA is visible during interphases (Fig. 38A). During mitosis the signal is reduced. Fig. 38B shows magnified images of time-lapse recordings. Early in interphase the signal intensity increases throughout the nuclei. Later in interphase bright spots appear in the nuclei representing active replication foci (indicated by arrows). At the end of $\mathrm{S}$ phase the nuclear signal diminishes. GFP-PCNA accumulated in the nuclei of X238 embryos and formed replication foci similar to WT embryos. However, GFP-PCNA persisted in X238 embryos of cycle 13 and became accumulated at the nuclear periphery, similar to DNA and EdU staining. 
A

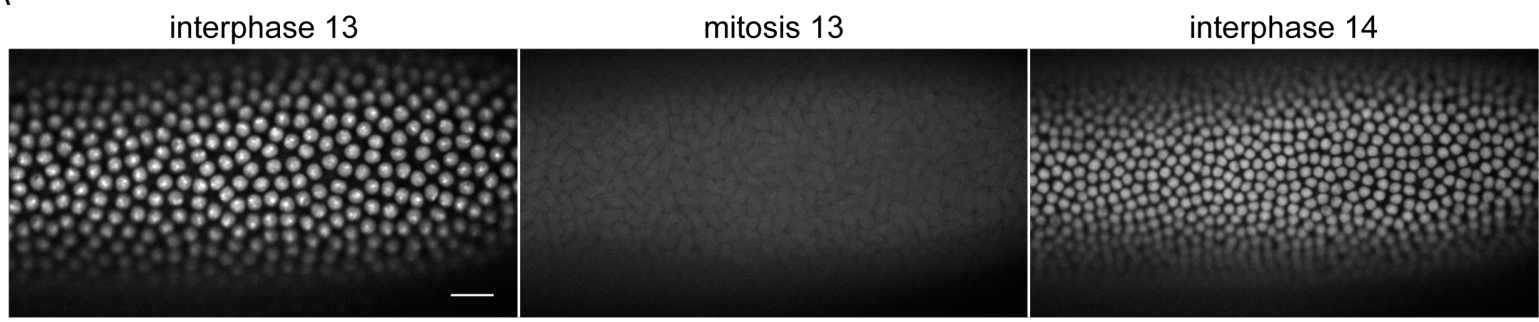

B
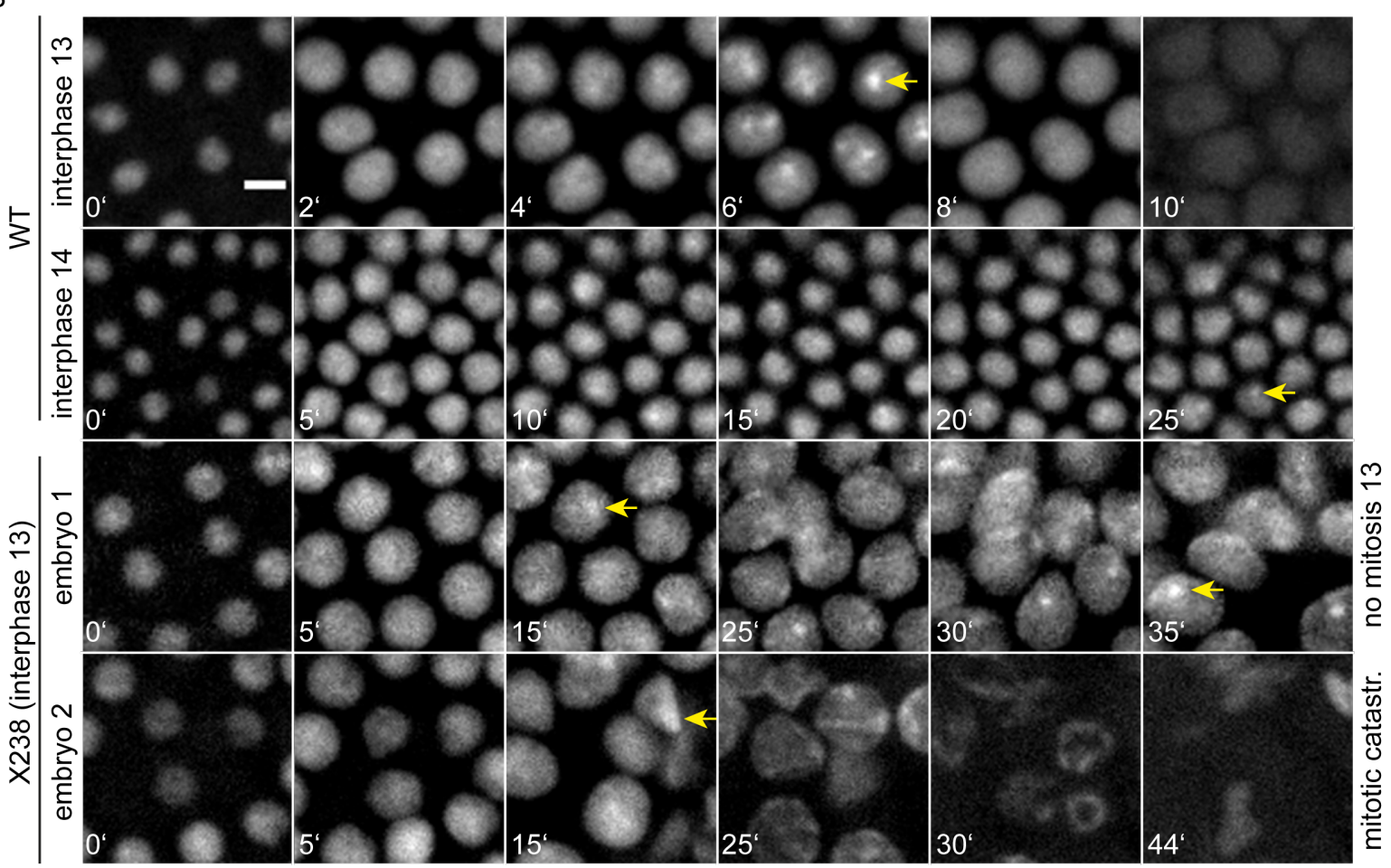

Figure 38: X238 embryos accumulate PCNA at the nuclear periphery. WT and X238 embryos expressing GFP-PCNA were imaged in time-lapse recordings. (A) In WT embryos GFP-PCNA was located in the nuclei in interphase and degraded in mitosis. Scale bar: $100 \mu \mathrm{m}$. (B) Magnified images of time-lapse recordings. In WT the onset of S phase was marked by a diffuse nuclear staining of GFP-PCNA, which became brighter and restricted to spots (replication foci, depicted with arrows) during later S phase. PCNA signal decreased at the end of S phase. In X238 mutant embryos PCNA foci were similar to WT but the GFP-PCNA signal was accumulated at the nuclear periphery. Scale bar: $5 \mu \mathrm{m}$. 
Table 15: Number of syncytial divisions is not changed upon overexpression of PCNA.

\begin{tabular}{lcrrrrrr}
\hline & & & \multicolumn{5}{c}{ Number of nuclei in $\mathbf{5 0} \mu^{\mathbf{2}}$ in cycle } \\
\cline { 5 - 9 } Genotype & Cycles & $\mathbf{n}$ & $\mathbf{1 0}$ & $\mathbf{1 1}$ & $\mathbf{1 2}$ & $\mathbf{1 3}$ & $\mathbf{1 4}$ \\
\hline WT & 14 & 4 & $5.5 \pm 0.5$ & $9.5 \pm 0.5$ & $19.5 \pm 2.8$ & $39.8 \pm 3.3$ & $74.8 \pm 1.3$ \\
X238Frt19A & 13 & 15 & $5.1 \pm 0.9$ & $9.2 \pm 1.6$ & $20.3 \pm 3.1$ & $37.1 \pm 3.6$ & \\
\hline WT + PCNA & 14 & 13 & $5.3 \pm 0.9$ & $11.0 \pm 1.7$ & $21.1 \pm 3.0$ & $40.5 \pm 3.6$ & $73.5 \pm 4.0$ \\
X238 + PCNA & 13 & 10 & $5.0 \pm 0.0$ & $10.3 \pm 0.4$ & $21.0 \pm 1.3$ & $36.4 \pm 4.5$ & \\
\hline
\end{tabular}

Table 16: Overexpression of PCNA does not significantly change the length of syncytial cell cycles.

\begin{tabular}{lrrrrrr}
\hline & & & \multicolumn{4}{c}{ Cell cycle length (min) } \\
\cline { 5 - 8 } Genotype & Cycles & $\mathbf{n}$ & \multicolumn{1}{c}{$\mathbf{1 0}$} & \multicolumn{1}{c}{$\mathbf{1 1}$} & $\mathbf{1 2}$ & $\mathbf{1 3}$ \\
\hline WT & 14 & 6 & $11.8 \pm 0.8$ & $12.5 \pm 0.5$ & $15.0 \pm 0.7$ & $21.8 \pm 0.9$ \\
X238Frt19A & 13 & 12 & $9.7 \pm 0.6$ & $10.6 \pm 1.1$ & $15.4 \pm 1.9$ & \\
\hline WT + PCNA & 14 & 10 & $11.0 \pm 0.0$ & $11.4 \pm 0.5$ & $15.3 \pm 1.7$ & $23.3 \pm 1.8$ \\
X238Frt19A + PCNA & 13 & 5 & & $9.5 \pm 0.5$ & $13.8 \pm 1.0$ & \\
\hline
\end{tabular}

\section{X238 mutants develop replication stress}

Ongoing replication without overreplication of chromosomes indicated replication stress, a problem in performing replication itself. Recently, Blythe et al. published an experimental method to visualise replication stress [28]. RpA70 accumulates at single-stranded DNA, which is formed at the replication fork during S phase, and can persist especially when there is interference between replication and transcription. We wanted to know, if X238 embryos develop replication stress and overexpressed RpA70-eGFP in these embryos. In WT RpA70-eGFP was located throughout the nucleus during interphase. Early cycles of X238 behaved similarly to WT. However, in cycle 13 RpA70-eGFP was accumulated as spots in the nuclei and at the nuclear periphery similar to DAPI, EdU, and PCNA staining. This result indicated that X238 accumulates replication stress during S phase 13. 


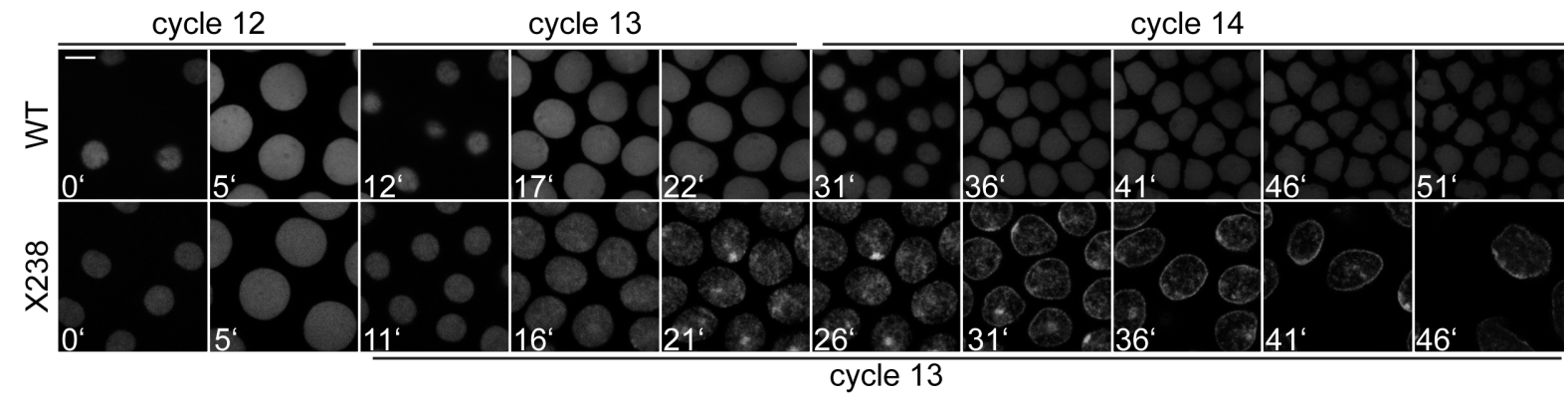

Figure 39: Replication stress occurs in cycle 13 in X238 embryos. Images from timelapse recordings of WT and X238 embryos expressing RpA70-eGFP. The single-strand DNA binding protein RpA70-eGFP was located in the interphase nuclei of WT and X238 embryos. RpA70-eGFP foci, which are an indicator for replication stress, were only formed in interphase 13 of mutant embryos. Scale bar: $5 \mu \mathrm{m}$.

\section{Centrosome duplication is not affected in X238 mutants}

As published in Nigg et al., 2011, centrosome duplication is coupled to S phase [109]. To address, if X238 embryos have a problem in centrosome duplication, which could lead to the detachment of the nuclei from the cortex, immunostaining against $\gamma$-Tubulin was performed. In syncytial interphases a pair of centrosomes is located apically to each nucleus. The centrosomes separate from each other and move to opposite sites of the nuclei as prophase starts. In X238 the number of centrosome pairs corresponded to the number of nuclei excluding that defect centrosome proliferation could induce the nuclear detachment.

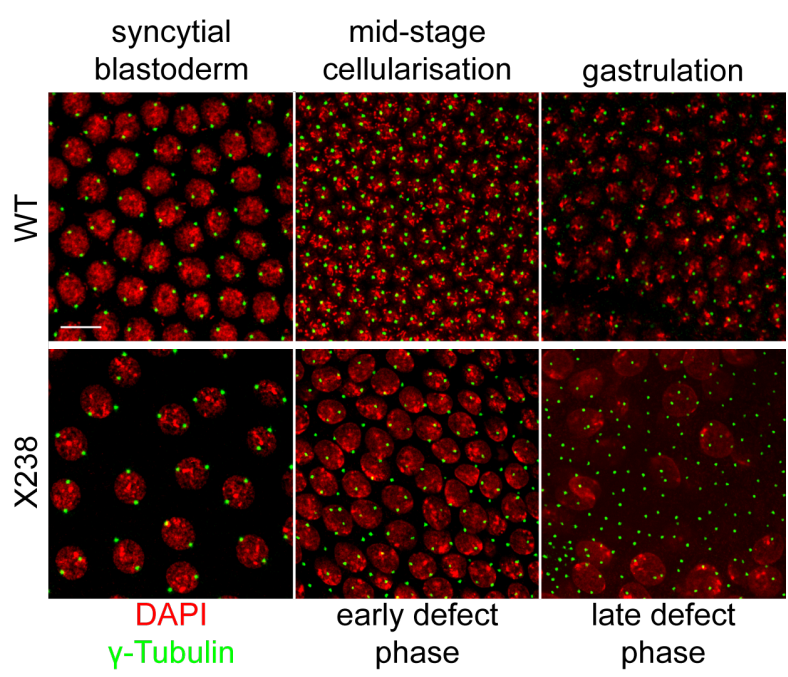

Figure 40: Centrosome proliferation is not affected in the mutant. WT and X238 embryos were immunostained with the centrosome marker $\gamma$-tubulin and the DNA marker DAPI. Centrosome duplication was coupled to the cell cycle and occurred in S phase. X238 embryos did not show a significant different centrosome density compared to WT. In late defect phase, where the nuclei were detached from the cortex, the connection of centrosomes and nuclei got lost. Images were taken with multiple $\mathrm{Z}$ layers. For visualisation, different $\mathrm{Z}$ layers for centrosomes and nuclei were merged. Scale bar: $10 \mu \mathrm{m}$. 


\subsection{Nuclear localisation of SHMT}

The X238 mutation causes a premature stop codon in the $\mathrm{C}$ terminus of the shmt gene. To identify the effect of this mutation on the protein level, antibodies were generated in rabbits and guinea pigs. In Western blot both antibodies detected a major band with a size of appr. $50 \mathrm{kDa}$ corresponding to the estimated molecular weight of SHMT (Fig. 41). Therefore we concluded this protein band represents SHMT. In X238 and M281R alleles this signal was reduced. M281R embryos had a weaker SHMT signal than X238. Introducing SHMT-rescue into the X238 mutant background restored the protein level to WT amount. An additional protein band was detectable at appr. $45 \mathrm{kDa}$. This band was more abundant in the mutant forms and disappeared in the rescued X238 mutant. So we concluded that it could represent a shorter peptide of SHMT.

Immunostaining of WT embryos with the SHMT antibody revealed a mainly cytosolic localisation throughout the embryo, which was reduced to background levels in X238 mutants (Fig. 42 and 43). This localisation pattern was maintained until stage 10, where the staining became restricted to a structure, which could reflect the Malpighian tubules [73] (Fig. 43).

Experiments in mammalian cell cultures systems revealed SHMT being translocated to the nucleus upon SUMOylation [6]. Marvin Uhlig analysed the nuclear localisation of SHMT in S2 cells during his bachelor work. He could detect a nuclear signal of SHMT in immunostainings after extraction of the nuclei from the cytoplasm (Fig. 44).

\subsection{Injection of GFP-HP1 rescues the cellularisation and cell cycle phenotypes of $\mathrm{X} 238$}

As described in Figure 36, X238 accumulated DNA at the nuclear periphery. One hypothesis to explain this phenomenon was that differentiation in eu- and heterochromatin is disturbed. To test this, GFP-tagged HP1, a heterochromatin binding protein essential for formation and maintenance of heterochromatin [110], was purified, injected into embryos and their development observed. Surprisingly, injected X238 embryos passed through 14 division cycles and finished cellularisation successfully (Fig. 45 and 46, Tables 17 and 18). Germband extension started and development proceeded at least until stage 8. Rescue of both cell cycle and cellularisation phenotype was dependent on the concentration of injected GFP-HP1, although with different dependencies (Tables 17 and 18). The effect was specific to HP1, as injection of GFP alone did not alter the blastoderm defect.

The rescue effect indicated indeed a problem in separation of eu- and heterochromatin, but we were unable to address the situation in the mutant with this approach. Embryos 
A

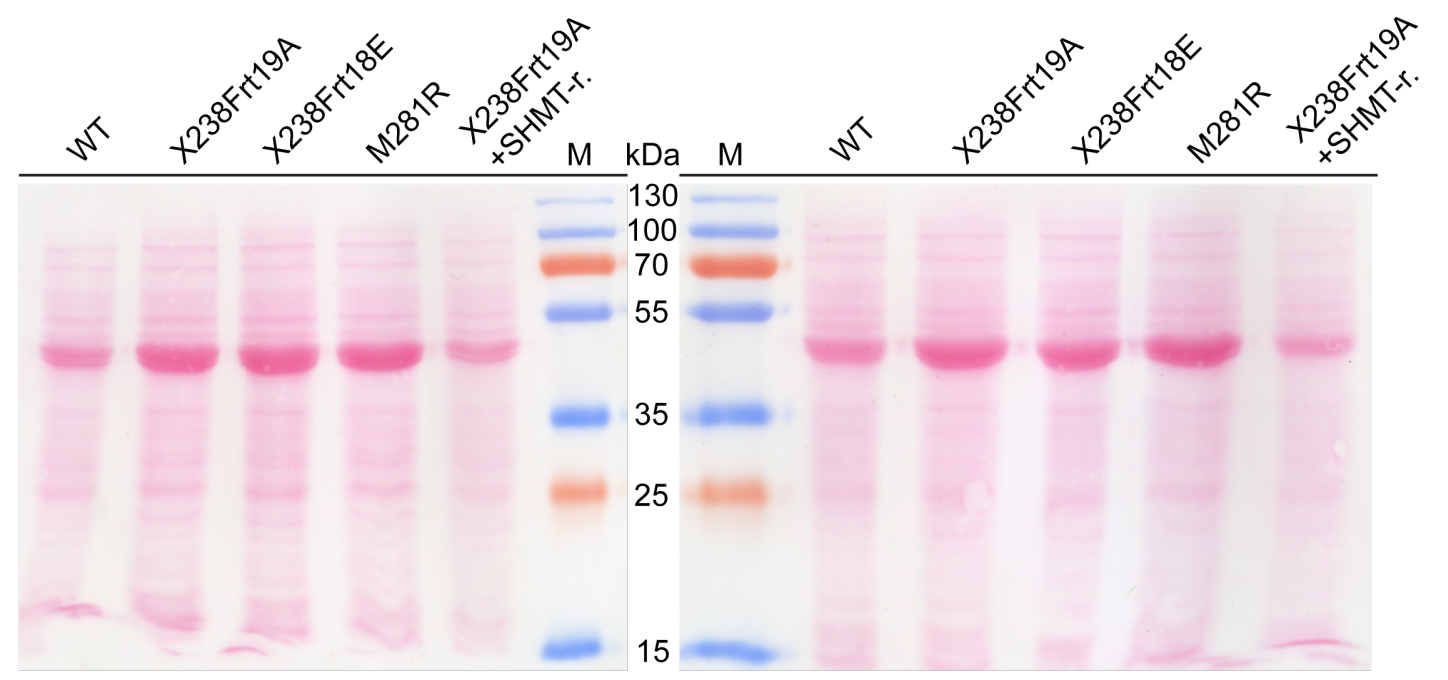

B

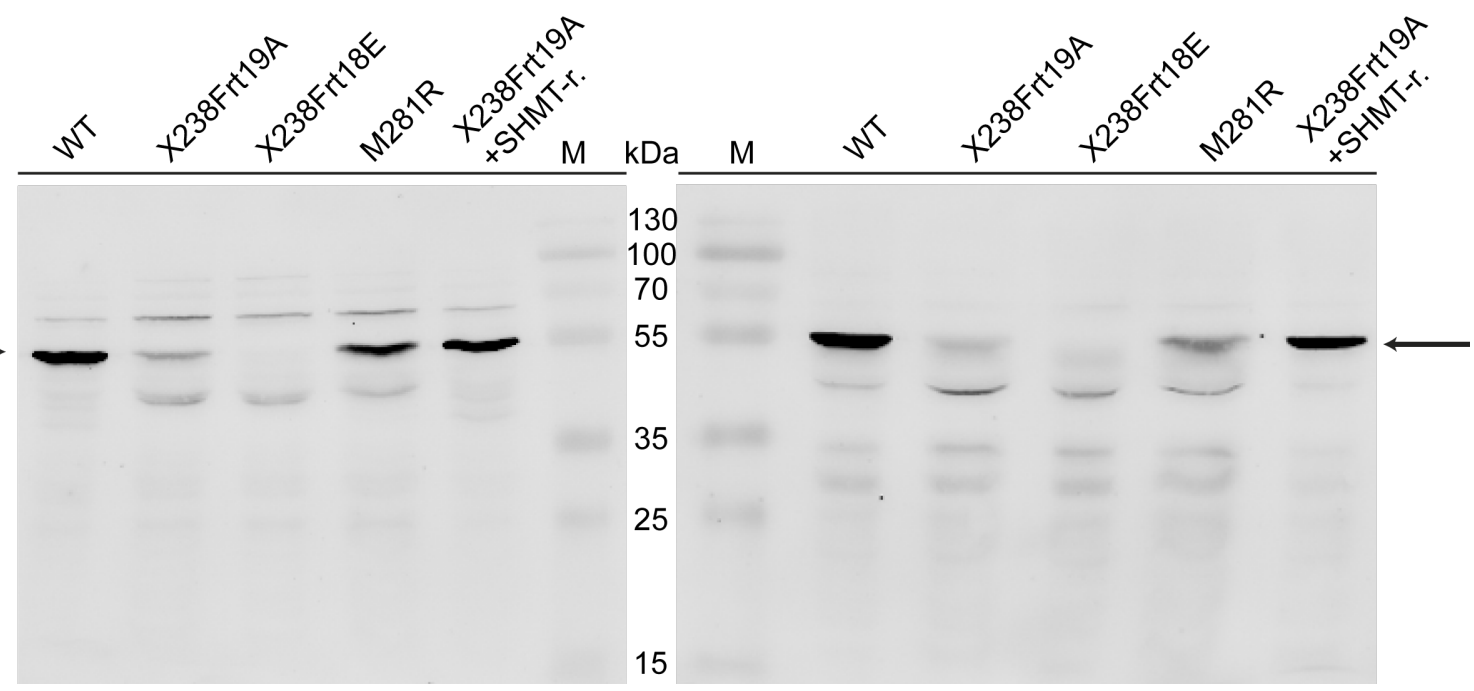

Figure 41: SHMT signal is reduced in shmt mutants. Western blot images (B) of SHMT antibodies generated in rabbits (left panel) and guinea pigs (right panel) with corresponding Ponceau stainings as loading controls (A). SHMT was detected at $\sim 50 \mathrm{kDA}$. Size and signal intensity were reduced in X238 mutants. M281R embryos showed a reduction in signal intensity for SHMT, which was not as prominent as for X238. Expression of the full rescue construct (SHMT-r.) restored SHMT expression in X238 to WT level.

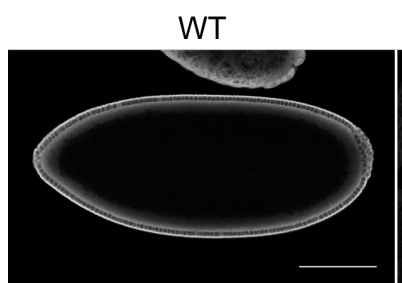

$\mathrm{X} 238$

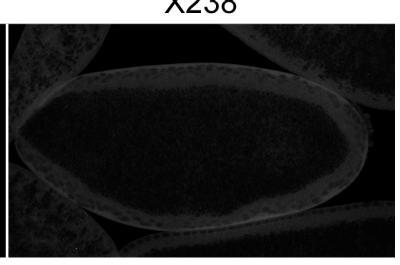

Figure 42: Antibody staining is specific for SHMT. Immunostaining against the SHMT protein with an antibody generated in guinea pigs. In X238 the level of SHMT is reduced to background levels compared to the cytosolic signal in WT. Scale bar: $100 \mu \mathrm{m}$. 


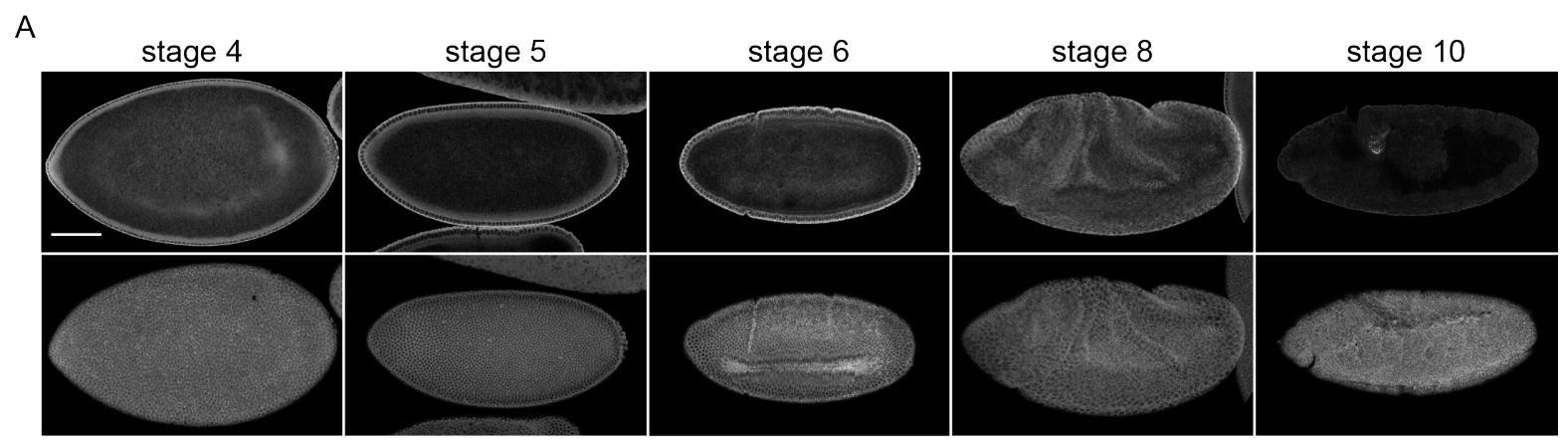

B

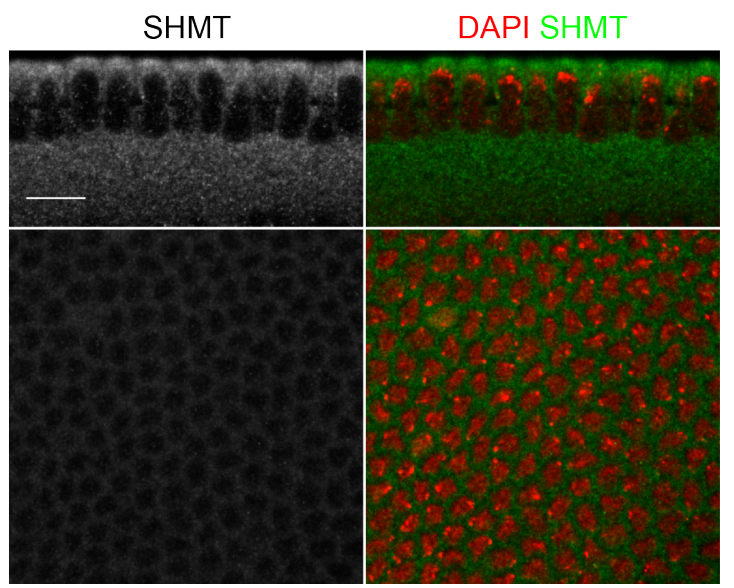

Figure 43: SHMT is localised predominantly in the cytosol. (A) Images of whole WT embryos stained with an SHMT antibody from rabbits. The upper panel shows a side view, the lower a surface view of the embryos. SHMT was uniformly localised in the embryo until stage 10, when it became restricted to a Malpighian tubules-like structure. Scale bar: $100 \mu \mathrm{m}$. (B) Magnified image showing the cytosolic localisation of SHMT during cellularisation. Scale bar: $10 \mu \mathrm{m}$.

were immunostained with an antibody generated against GFP-HP1. This polyclonal antibody detected both GFP and HP1 proteins. In early WT and X238 embryos HP1 was located throughout the nuclei (Fig. 47). During cellularisation HP1 became restricted to the apical heterochromatin. In X238 embryos showing the first signs of defects the staining was different compared to WT. HP1 did not exhibit any restriction to specific parts in the nuclei, but was rather absent in several nuclei. This staining persisted in late defect embryos. The staining further supported the hypothesis of a failure in formation and / or maintenance of eu- and heterochromatin. 


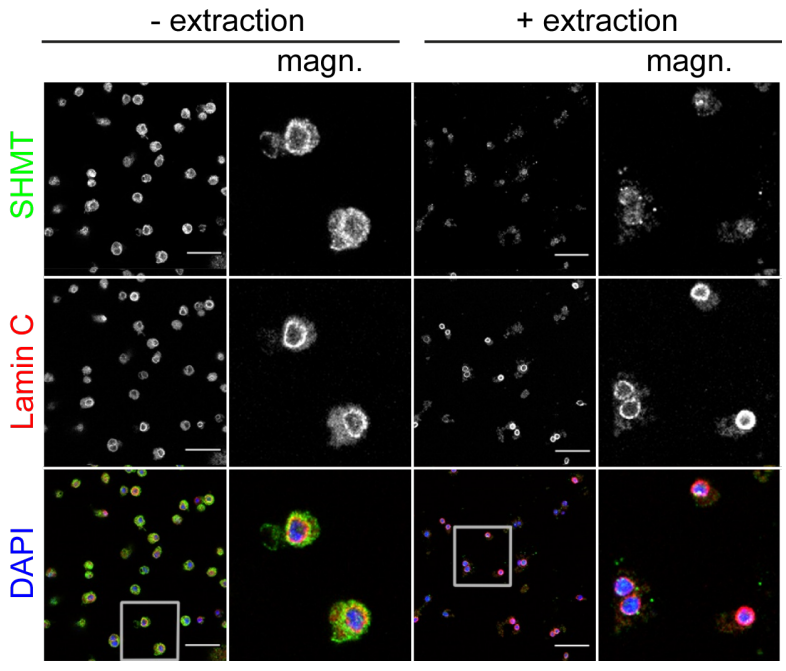

Figure 44: Immunofluorescent stainings of S2 insect cells showing the localisation of SHMT and Lamin C before and after extraction. Without extraction the signal of SHMT was mainly cytosolic (left panels). After extraction a nuclear staining of SHMT was observed (right panels). A square box marks magnified parts of the image. Scale bar: $20 \mu \mathrm{m}$. (Experiment and imaging were carried out by M. Uhlig [162]mod.).

Table 17: Injection of GFP-HP1 rescues the cell cycle phenotype of X238.

\begin{tabular}{lcccccc}
\hline & \multicolumn{7}{c}{ Syncytial cycles (\%) } \\
\cline { 2 - 6 } Treatment & $\mathbf{1 2}$ & $\mathbf{1 2} \backslash \mathbf{1 3}$ & $\mathbf{1 3}$ & $\mathbf{1 3} \backslash \mathbf{1 4}$ & $\mathbf{1 4}$ & $\mathbf{n}$ \\
\hline uninjected & 2.5 & 5 & 72.5 & 7.5 & 12.5 & 40 \\
HP1 - undiluted & 0 & 0 & 34.8 & 13 & 52.2 & 23 \\
HP1 - 1:5 & 0 & 0 & 75 & 0 & 25 & 12 \\
HP1 - 1:10 & 0 & 0 & 71.4 & 4.8 & 23.8 & 21 \\
HP1 - 1:20 & 0 & 0 & 82.4 & 11.8 & 5.9 & 17 \\
GFP & 10 & 0 & 90 & 0 & 0 & 10 \\
\hline
\end{tabular}

Table 18: Cellularisation defect of shmt mutants can be rescued by injection of GFP-HP1.

\begin{tabular}{lrccr}
\hline & \multicolumn{3}{c}{ Cellularisation (\%) } & \\
\cline { 2 - 4 } Treatment & \multicolumn{1}{c}{ no } & partly & complete & n \\
\hline uninjected & 67.5 & 15 & 17.5 & 40 \\
HP1 - undiluted & 8.7 & 17.4 & 73.9 & 23 \\
HP1 - 1:5 & 41.7 & 25 & 33.3 & 12 \\
HP1 - 1:10 & 42.9 & 28.6 & 28.6 & 21 \\
HP1 - 1:20 & 47.1 & 29.4 & 23.5 & 17 \\
GFP & 100 & 0 & 0 & 10 \\
\hline
\end{tabular}




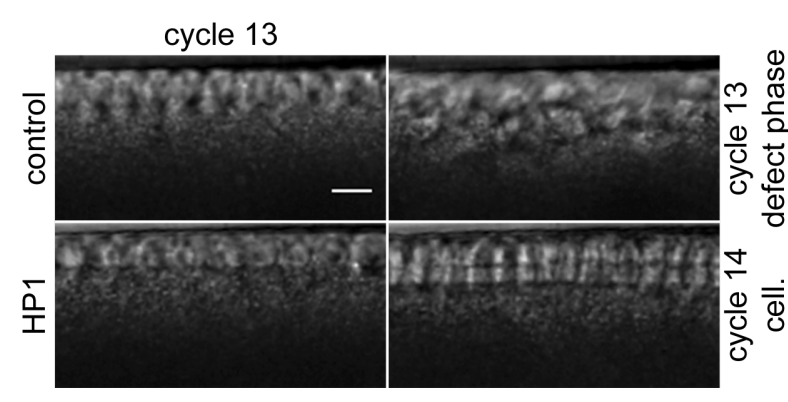

Figure 45: X238 mutant embryos injected with GFP-HP1 progress into cycle 14 and undergo cellularisation. WT and X238 embryos injected with GFP-HP1 were imaged by time-lapse recording using DIC optics. Whereas injection into WT did not alter development, X238 embryos could overcome their cell cycle and cellularisation phenotypes upon injection with GFP-HP1. Scale bar: $10 \mu \mathrm{m}$.
A

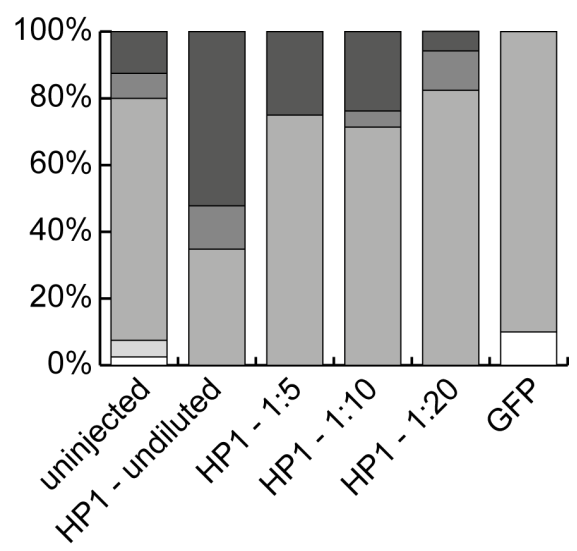

B

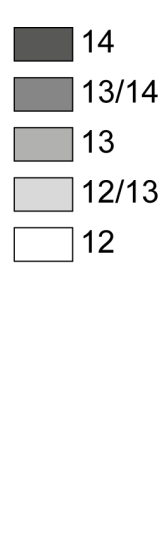

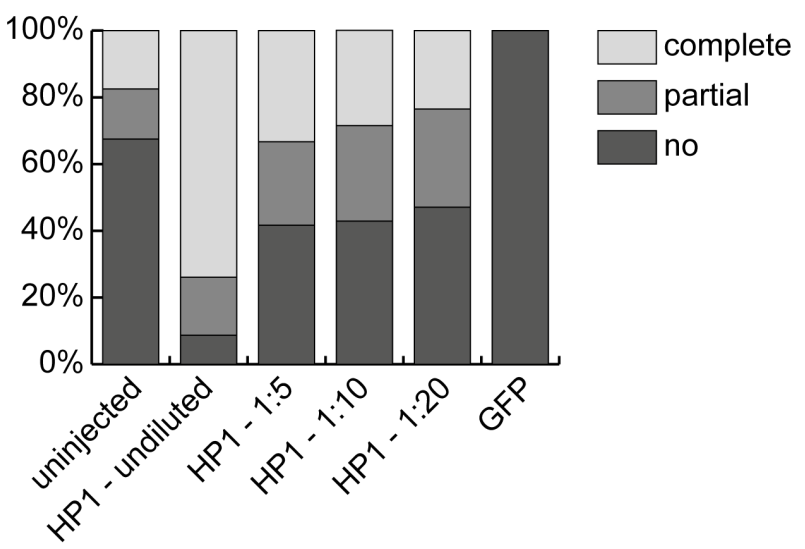

Figure 46: Effect of different concentrations of injected GFP-HP1 on the cellularisation phenotype of X238. X238 embryos were injected with different concentrations of GFP-HP1 or GFP alone and recorded by DIC imaging. The number of syncytial cell cycles (A) and cellularisation ability (B) was determined from the time-lapse recordings as described in Figure 21. (A) Upon injection of highly concentrated GFP-HP1 a higher percentage of the X238 embryos progressed into cycle 14 compared to uninjected embryos ( $52.2 \%$ compared to $12.5 \%$ ). This effect decreased gradually upon reduction of the GFP-HP1 concentration. Injection of GFP alone had no effect on the cell cycle phenotype of X238. (B) Similar to the effect on the cell cycle phenotype, injection of highly concentrated GFP-HP1 rescued the cellularisation defect in the majority of the injected embryos ( $73.0 \%$ compared to $12.5 \%$ of uninjected embryos). The rescue ability decreased with decreasing GFP-HP1 concentration, although it was decreasing not as strong as the decrease of the cell cycle rescue ability. Injection of GFP alone did not alter cellularisation progression. The bar charts visualise the data presented in tables 17 and 18. 


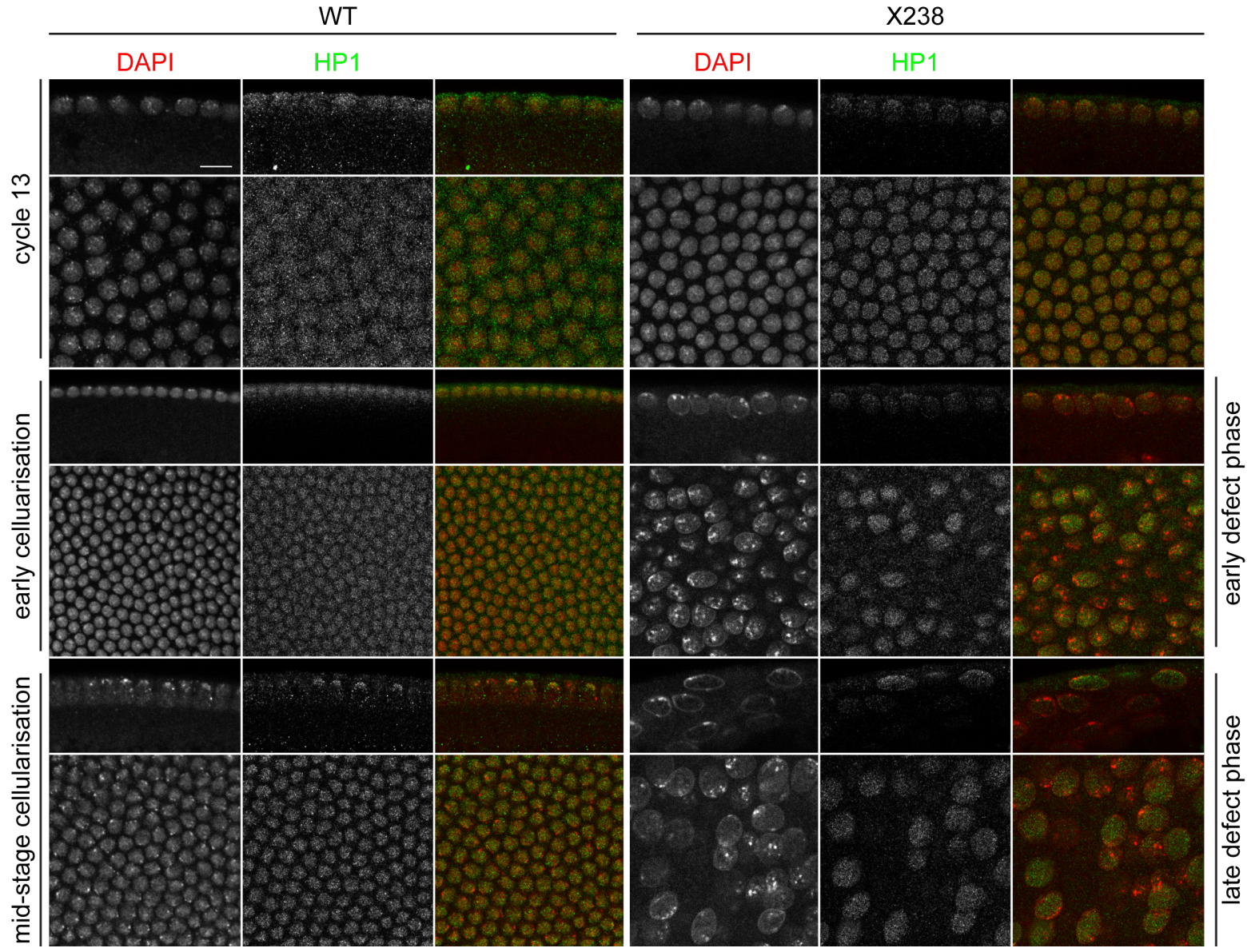

Figure 47: HP1 staining becomes irregular in X238 embryos. Immunostaining of WT and X238 embryos with an antibody against GFP-HP1 and the DNA dye DAPI. HP1 located throughout the nuclei in syncytial nuclei in WT, but became restricted to the apical heterochromatin during cellularisation. In X238 embryos early in cycle 13 a similar staining could be observed. However, in the defect phase nuclei without HP1 signal were observed. Nuclei with HP1 staining exhibited a staining pattern similar to WT nuclei of early cycle 14. Scale bar: $10 \mu \mathrm{m}$. 


\subsection{Gene expression in X238 embryos}

In order to identify differences in gene expression, which could contribute to the X238 defect, RNA sequencing and Nanostring experiments were performed. For both approaches RNA extracted from precisely staged embryos was used. Embryo collection and RNA extraction were done by Dr. Hung-wei Sung. RNA sequencing was carried out by the Transcriptome Analysis Laboratory (TAL), which also provided Figures 48, $53,54,55,56$ and 57$)$.

The obtained data displayed a high quality. The range of reads layed between appr. 22 and 37 millions. More than $90 \%$ of these could be annotated to appr. 8000 to 10000 genes per sample. RNA sequencing data samples were analysed according to their clustering behaviour to determine how similar one sample type was to each other compared to other sets. In Fig. 48 the clustering dendrograms for presyncytial (A) and blastoderm data sets (B) are shown. Data obtained from presyncytial WT and X238 samples clustered to each other without overlap. Data set X238-13-3, a sample from X238 in blastoderm stage, was more similar to the WT sample sets as to the other two X238 data sets. But due to the low overall differences between WT and X238 data sets, this sample was still taken into account for further analysis.

The Nanostring CodeSet provides transcripts as internal controls. These contain six positive controls with defined concentrations, and 8 negative controls without transcripts. Negative controls are used to determine the background level of Nanostring counting. In this experiment the maximum value obtained was 9 (Fig. 49A, Table 25). Positive controls can be used to determine the accuracy of the measurement. All controls behaved similarly between WT and X238 samples with decreasing count number from control POS_A (highest concentration) to POS_F (lowest concentration) (Fig. 49B).

A second control for Nanostring was carried out by including three housekeeping genes (Rpl21, RpLP2, and RpL32), which should not differ in their expression level throughout development or between WT and X238. As depicted in Fig. 50A the mRNA levels of all transcripts were stable throughout development. Values obtained for WT and X238 were also comparable (Fig. 50B). In this figure, the ratio between WT and X238 transcripts counts are shown. A value around 1 indicated no difference between the genotypes.

\subsubsection{Zygotic genome is activated in $\mathrm{X} 238$}

By using Nanostring technology we wanted to find out, if the zygotic control of development is activated. Transcript numbers of marker genes for zygotic expression and zygotically controlled degradation of maternally provided mRNAs were determined [151]. The analysed genes displayed the expected behaviour of activation or degradation, respectively, similar in WT and X238 samples (Fig. 51). In the following, exemplary 

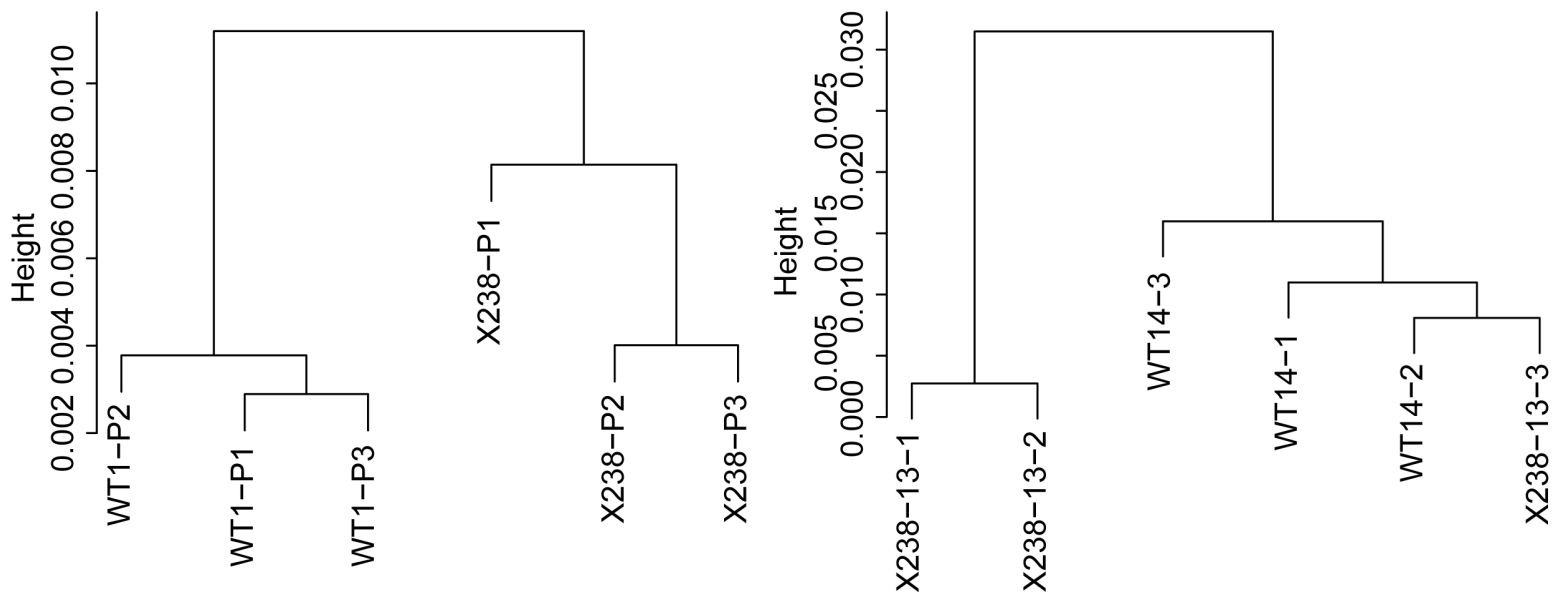

Figure 48: RNA samples of the same genotype cluster together. (A) Comparison of data obtained from presyncytial samples. WT and X238 formed separated clusters. (B) Comparison of data from blastoderm samples. Whereas the WT samples clustered together, sample X238-13-3 was more similar to the WT samples than to the other X238 samples.

A

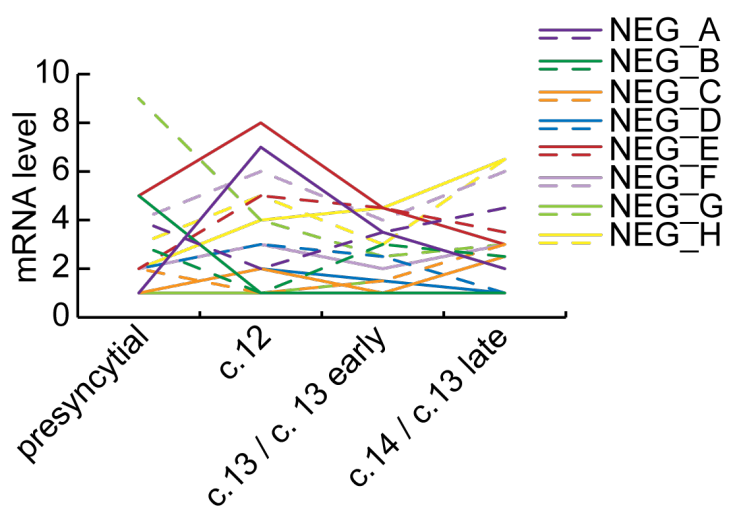

B

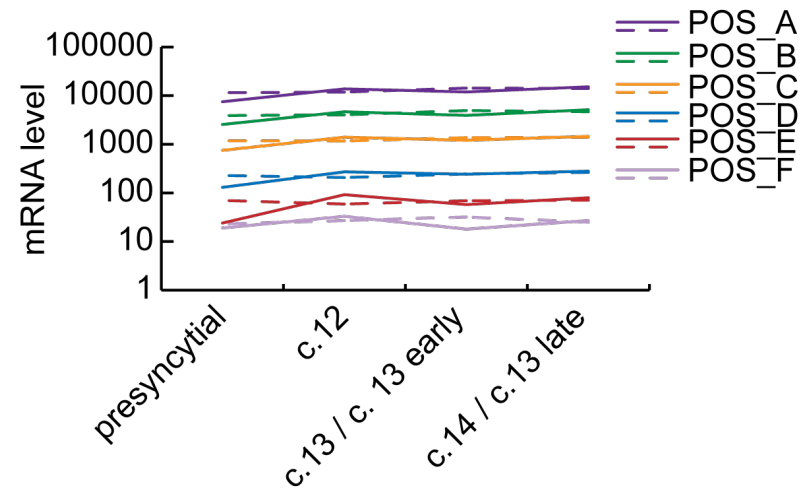

Figure 49: Internal Nanostring CodeSet controls for normalisation and background determination. (A) Counts obtained for Nanostring negative controls, which did not contain transcripts. (B) RNA levels for positive controls, which consisted of ERCC control transcripts of defined concentrations, allowing estimation of overall efficiency of hybridisation. Positive controls were included in the normalisation procedure. Continuous line: WT, dashed line: $\mathrm{X} 238$. 
A

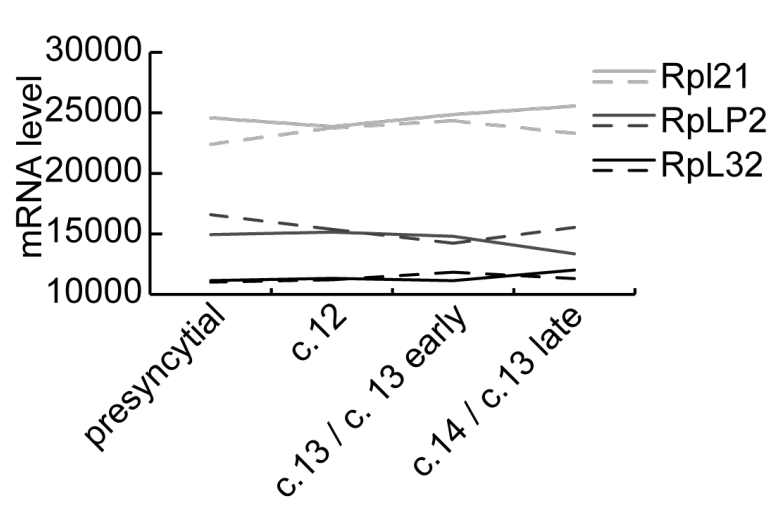

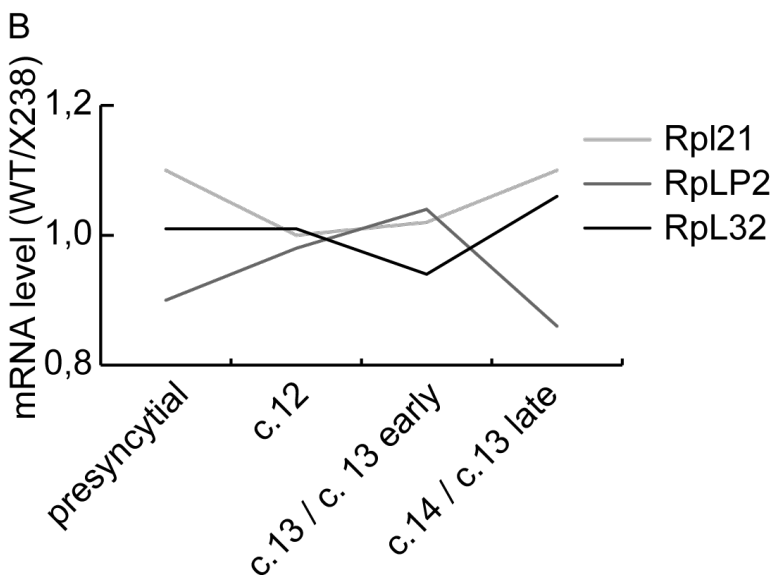

Figure 50: Expression of the housekeeping genes Rpl21, RpLP2, and RpL32 is similar between WT an X238. (A) Visualisation of normalised counts for transcripts. (B) Line chart of the ratio of the mRNA levels of WT and X238. Continuous line: WT, dashed line: X238.

genes were chosen and described in detail. As mentioned in section 2.4, Frs expression is important to stop the syncytial cell cycle program allowing cellularisation and MBT. Großhans and colleagues reported that Frs mRNA becomes expressed late in cycle 13 and peaks during cellularisation [69]. Although we detected already a low expression of Frs in cycle 12 WT embryos due to the higher sensitivity of Nanostring compared to in situ hybridisation, we could confirm that the Frs mRNA becomes highly expressed during cellularisation stage (Fig. 51A, continues orange line). The number of Frs transcripts in X238 was similar to WT embryos of the corresponding stages (Fig. 51A, dashed orange line and 51B, orange line). Eve is a pair-rule gene, which accumulates in WT embryos in seven stripes during cycle 14 [60]. Its mRNA was already detectable in WT embryos in cycle 12 and increased in the further cycles (Fig. 51A, continues dark green line). In X238 embryos Eve transcription was similar to WT although a higher transcript number was detected in presyncytial cycles (Fig. 51A, dashed dark green line and 51B, dark green line), confirming the data obtained for the protein localisation described in section 2.2.1 (Figure 14, right panels). The Drosophila homologues of Cdc25, String and Twine, promote mitotic divisions in the early embryonic development [42]. Both mRNA and protein of string and twine are zygotically degraded to stop the cell cycle progression in cycle 14. Nanostring measurement revealed that string and twine mRNA were degraded in syncytial blastoderm stage (Fig. 51C, continuous green and orange lines, respectively), whereby string degradation was initiated earlier than twine degradation. In X238 embryos string and twine were degraded in a similar time course to WT (Fig. 51C, continuous green and orange lines and 51D, green and orange lines, respectively). These data allowed the conclusion that in X238 embryos the switch to zygotic control of development is activated in late cycle 13 . 
A

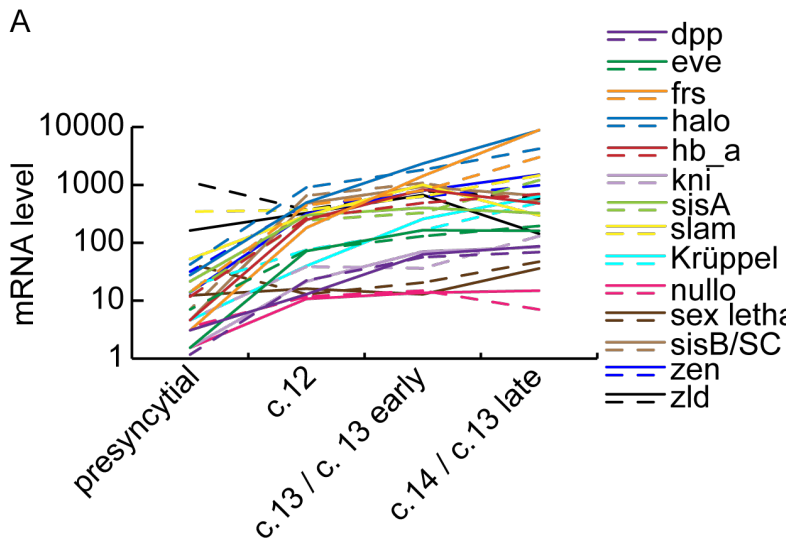

C

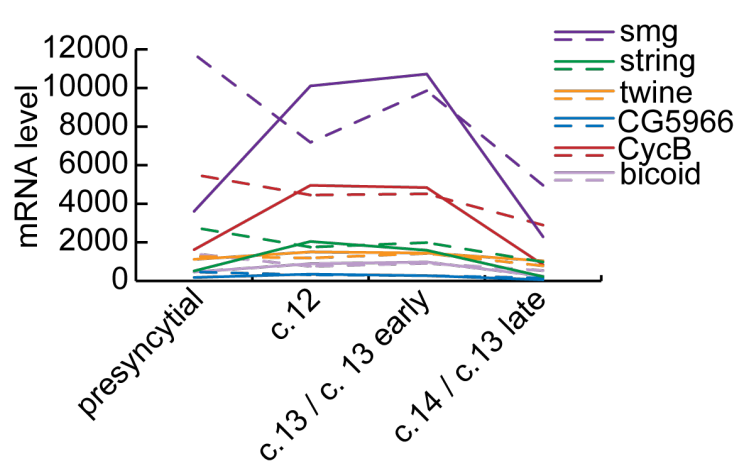

$\mathrm{B}$

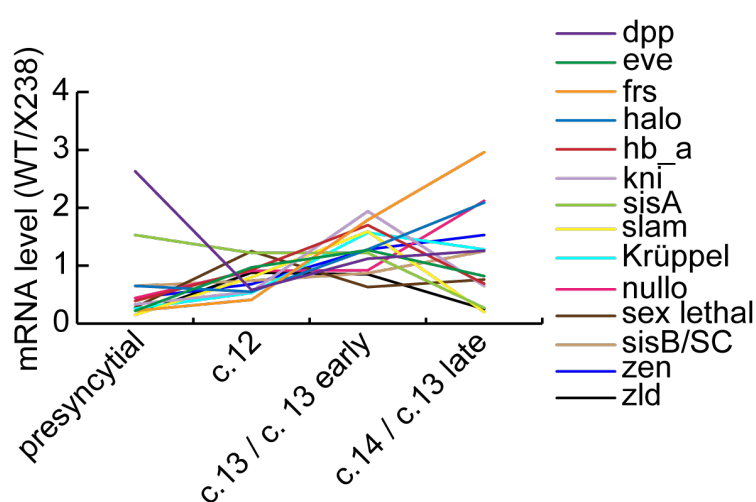

D

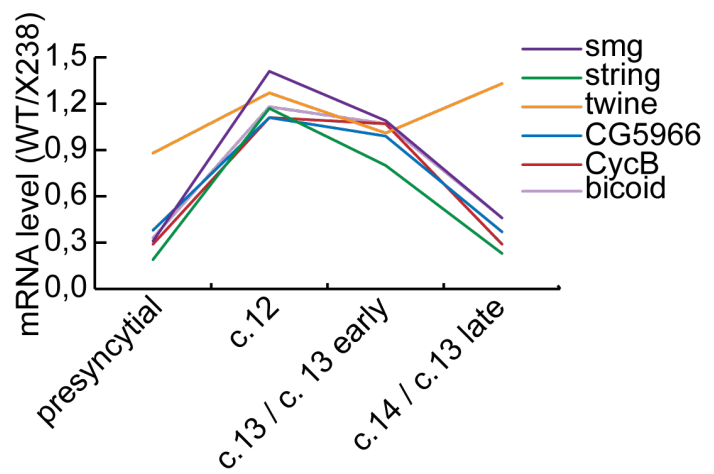

Figure 51: Expression of zygotic genes and zygotically controlled degradation of mRNA is activated in X238. (A) + (B) Visualisation of normalised mRNA counts (A) and the ratio values (B) of zygotically expressed genes. WT and X238 gene expression was activated similarly. mRNA level in presyncytial stage was low and increased gradually during syncytial blastoderm development, as could be seen for example in the genes Frs and Eve. (C) + (D) Line charts of normalised counts (C) and their ratio values (D) for mRNAs, which were zygotically degraded. In presyncytial stage the mRNAs levels were high compared to the late syncytial blastoderm stage. Although transcript numbers in presyncytial WT embryos were lower than the corresponding X238 values, their degradation behaviour was similar as could be seen for example for the genes string and twine. Continues line: WT, dashed line: X238. 


\subsubsection{Maternal RNA degradation is similar between WT and X238}

The degradation of maternal transcripts, which are not zygotically controlled, was analysed similar like zygotic gene activation. X238 embryos degraded maternal genes similar to WT embryos (Fig. 52). An example was nanos mRNA, which encodes a protein necessary for the formation of germ cells and abdomen (reviewed in Kobayashi et al., 2005 [82]) nanos is reported to be degraded at the MBT except for the posterior pole plasm and pole cells $[15,20,104]$. WT and X238 embryos showed a similar time-course of global nanos mRNA degradation in the Nanostring analysis (Fig. 52, orange lines), indicating that zygotically independent degradation of maternal RNA is initiated in X238 embryos.

A

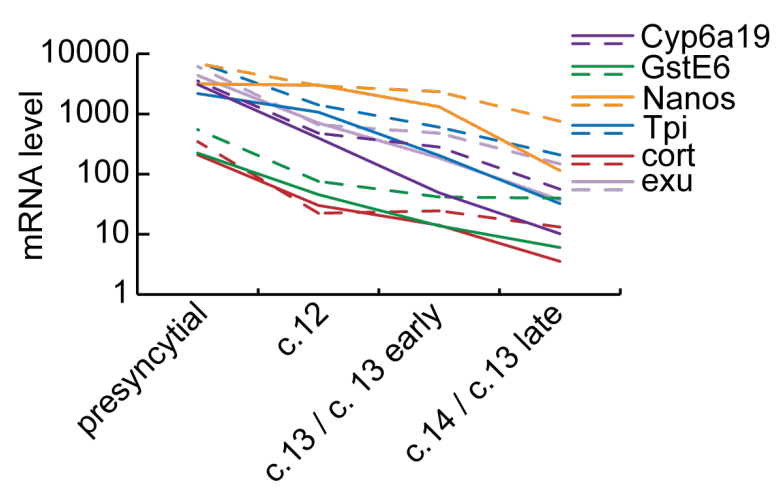

B

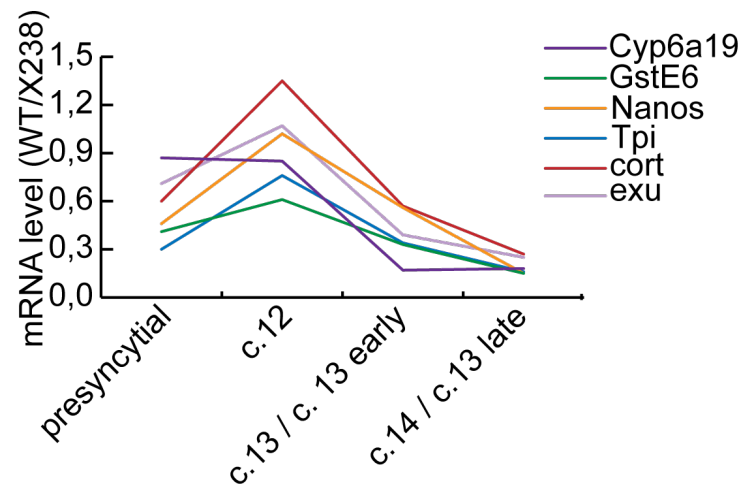

Figure 52: Maternal genes are degraded in X238. Visualisation of normalised mRNA counts (A) and the ratio values (B) of maternally degraded genes. WT and X238 embryos showed a similar degradation progress of the transcripts. Transcript numbers in presyncytial stage were high and were degraded during syncytial blastoderm development as could be seen for example for the nanos gene. Continues line: WT, dashed line: X238.

\subsubsection{X238 embryos show a specific change in their gene expression profile for metabolic enzymes}

The Nanostring experiment revealed that the major genes which are necessary for MBT were activated and degradation of maternal transcripts happened as expected. We wanted to further analyse global differences in gene expression, which could occur upon shmt mutation. To achieve this, RNA sequencing was performed.

First, gene expression of embryos in presyncytial stage was analysed. Fig. 53 shows the corresponding MA plot. This is a plot, where expression data are transformed onto the M (log ratios) and A (mean average) scale. RNAs with low expression values are located on 

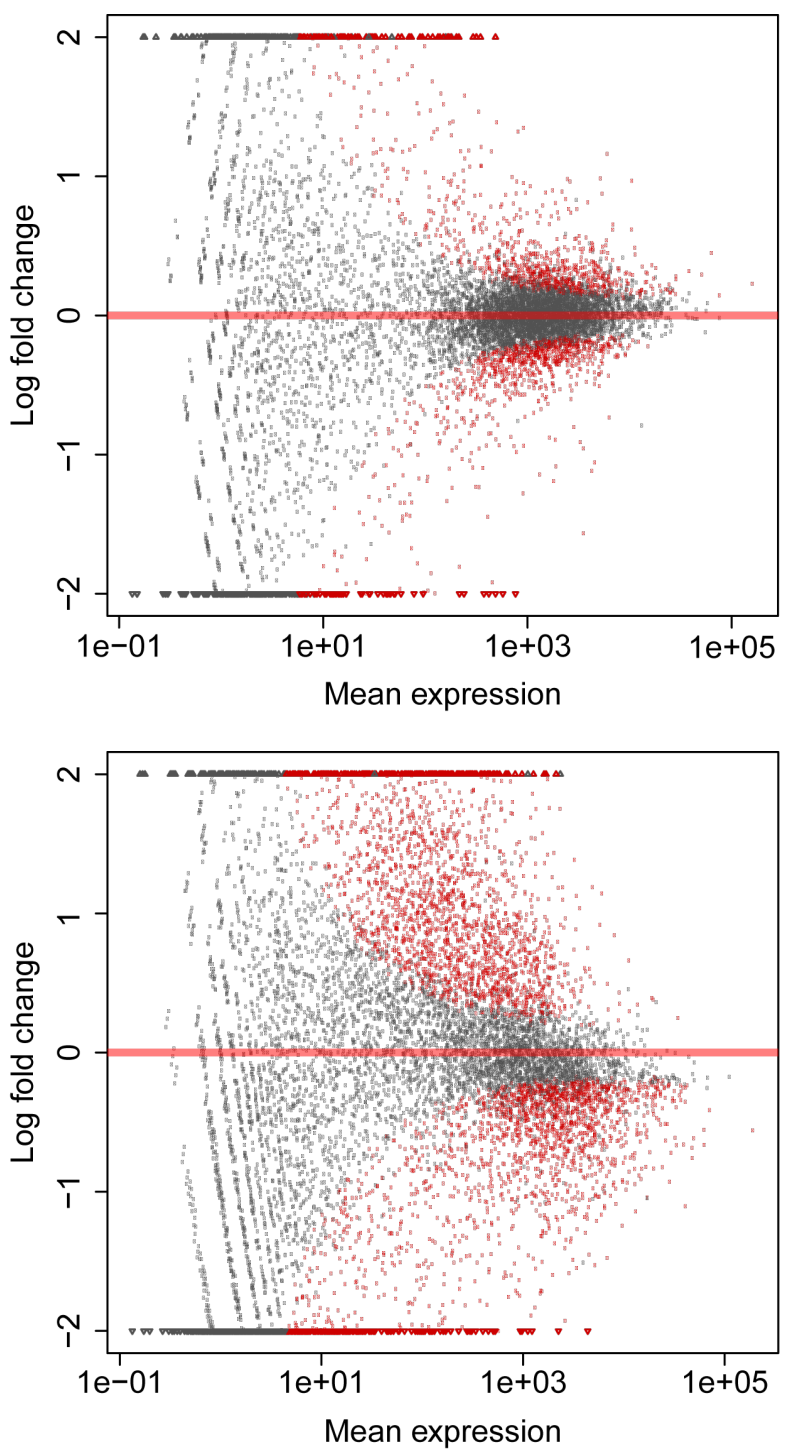

Figure 53: MA plot for comparison of presyncytial WT and X238 expression profiles. Mean expression of RNA transcripts plotted against log fold changes. Red dots: significantly differentially expressed genes, grey dots: similarly expressed genes.

Figure 54: MA plot for comparison of WT and X238 blastoderm stage expression profiles. Mean expression of RNA transcripts plotted against log fold changes. Red dots: significantly differentially expressed genes, grey dots: similarly expressed genes.

the left site, highly expressed RNAs on the right site. In red all transcripts are depicted, which were significantly differentially expressed, thus representing candidate genes. Fig. 56 provides a heat map with the 50 most significantly differentially expressed genes. For this list not only the absolute difference in expression were taken into consideration, but also if all samples of a sample group behaved similarly. A list of all 69 identified candidate genes is provided in the appendix (Table 30). These genes did not cluster in any gene ontology (GO) terms. Biological processes included for example neural development, organ development, gluthatione metabolism, oxidation-reduction processes, and various signalling pathways.

Second, we analysed gene expression of embryos in blastoderm stage. Fig. 54 displays the corresponding MA plot. The number of red dots, representing candidate genes, was increased compared to presyncytial stage. In total 1405 candidate genes were identified, 


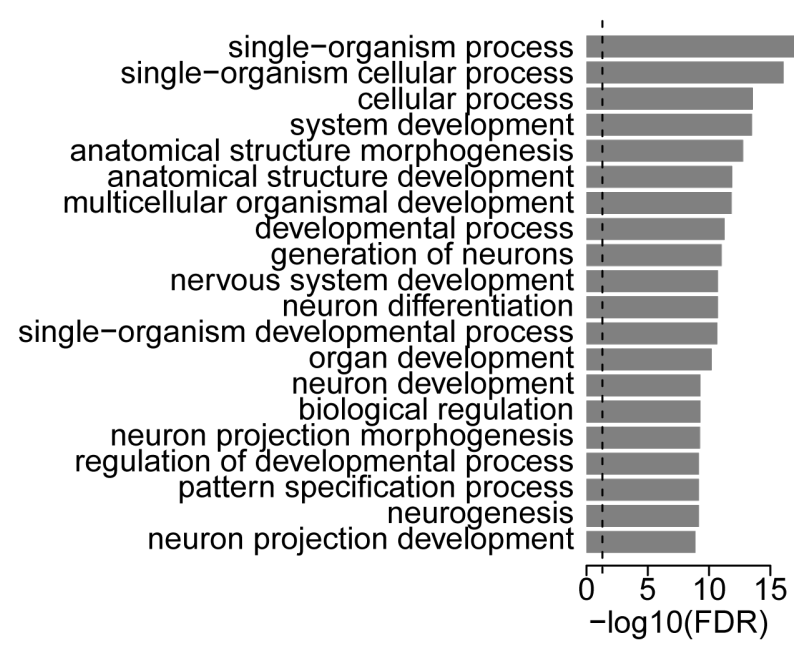

Figure 55: Most enriched GO terms of differentially expressed genes of blastoderm staged embryos.

which had an expression difference factor of minimal 2 and a false discovery rate (FDR) of maximal $5 \%$. The candidate genes were enriched in 268 GO terms, the 20 most enriched are depicted in Fig. 55. These GO terms belonged either to general or neural development. Fig. 57 shows the heat map with the 50 most significantly differentially expressed genes. A list of the 38 most up- and down-regulated genes is provided in the appendix (Table 31). Within this list several genes were found, which also appeared as candidates in presyncytial staged embryos (e.g. CG3038 and sawPsi). 


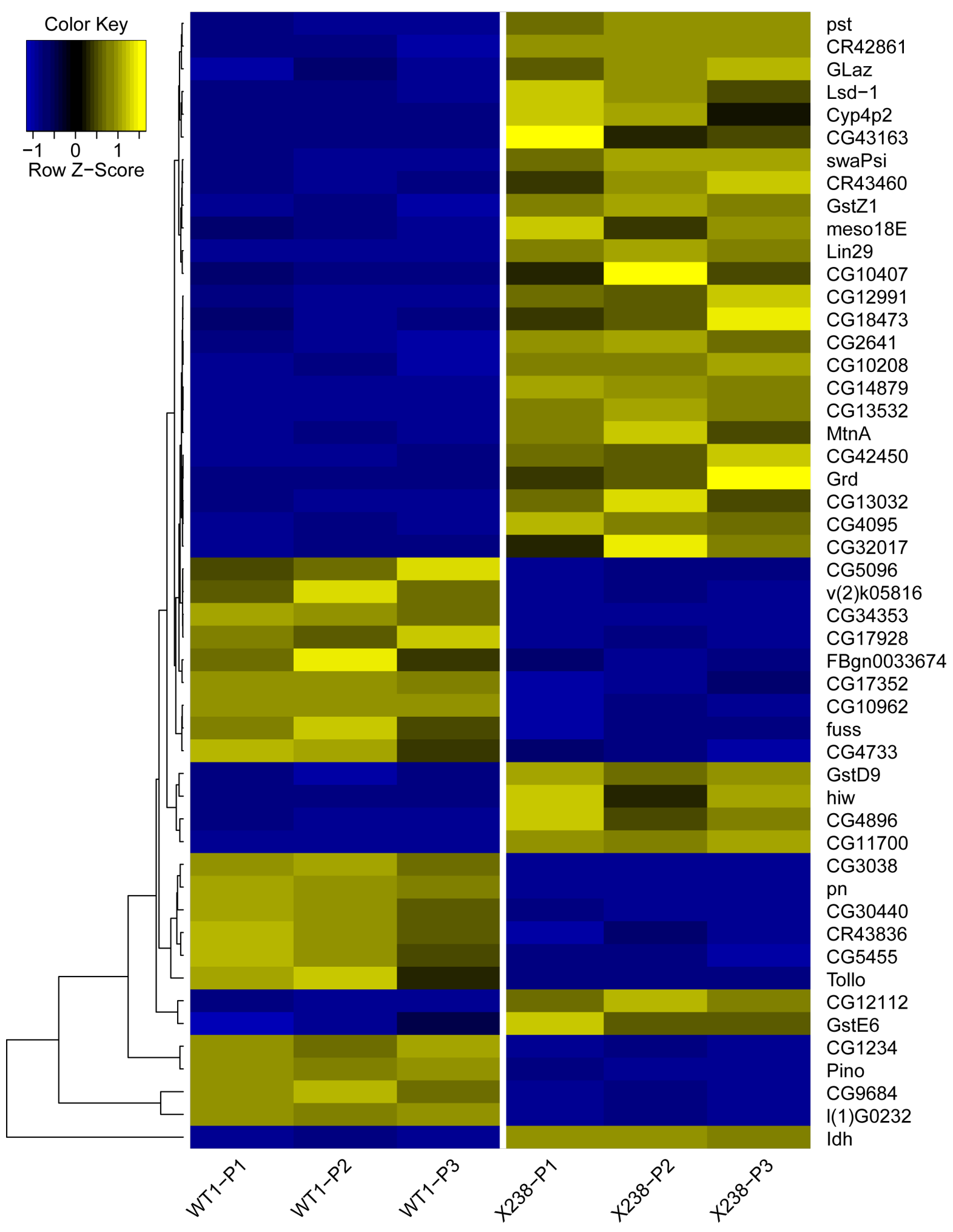

Figure 56: Heat map with the 50 most significantly differentially expressed genes in presyncytial staged embryos. Colour code: blue=low expression, yellow=high expression. 


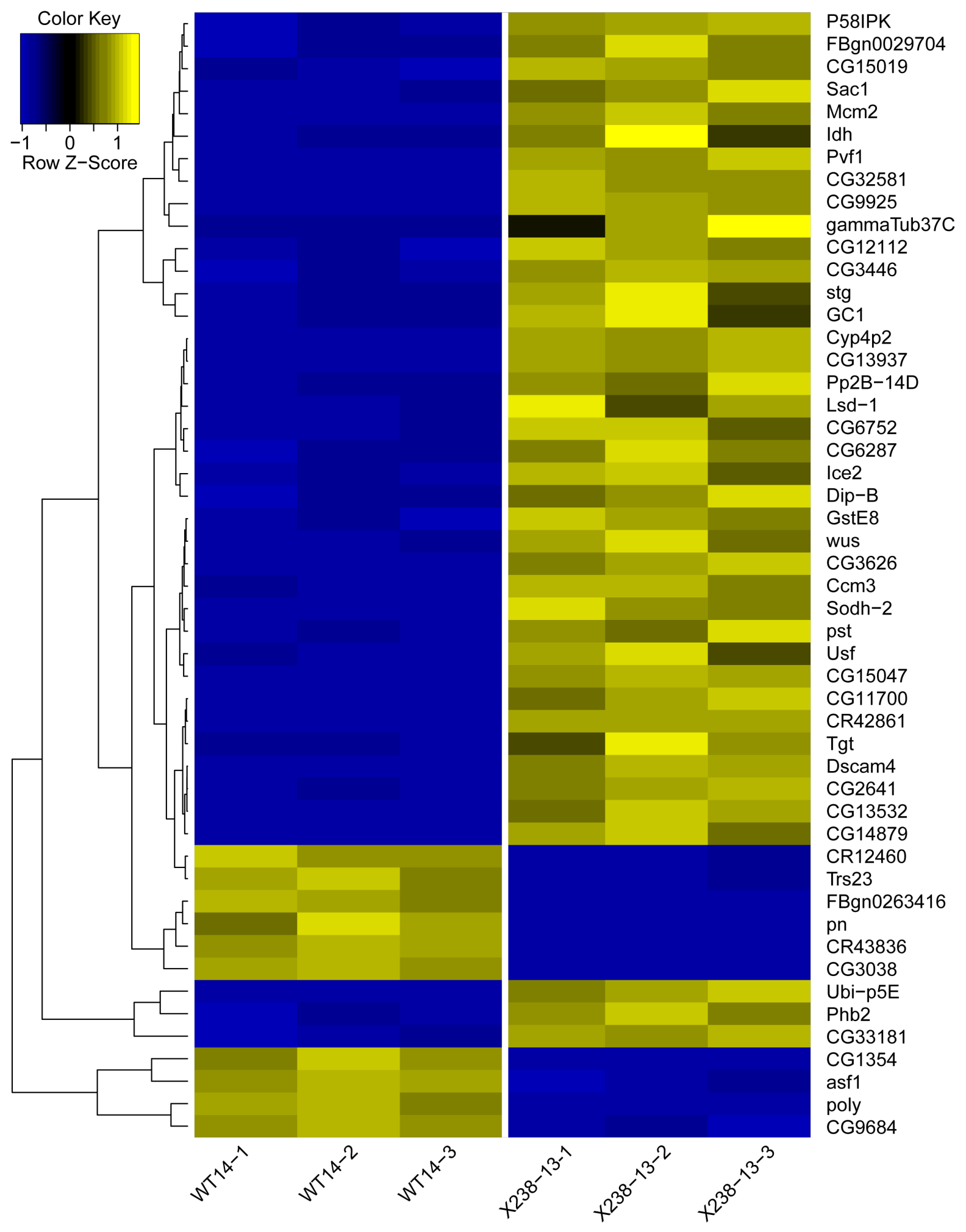

Figure 57: Heat map with the 50 most differentially expressed genes in blastoderm staged embryos. Colour code: blue=low expression, yellow=high expression. 



\section{Discussion}

The folate-mediated one-carbon metabolism is a network of connected anabolic pathways, which is required for the synthesis of thymidylate, purines and the remethylation of homocysteine to methionine, which can be converted into SAM, an important cofactor for methylation reactions of numerous compounds including DNA, RNA and proteins such as histones $[9,58,102]$. SHMT is a central enzyme in this network, catalysing the reaction of serine into glycine and THF into mTHF (Fig. 5). Although the importance of this reaction could imply that depletion of shmt would lead to cell death, Drosophila shmt mutant embryos from germline clones developed until syncytial blastoderm stage. Both mutant alleles X238 and M281R, which were analysed in this thesis, stopped their syncytial cell cycle program in cycle 13 and exhibited a cellularisation phenotype.

\subsection{The enzymatic function of SHMT is required for early Drosophila development}

SHMT is part of the thymidylate pathway, which synthesises thymidylate from dUMP and mTHF (Figure 6) [74]. Experiments in mice and mammalian cell culture revealed that shmt deficiency, caused either by mutation or by folate deficiency, leads to an increased incorporation of uracil into the DNA, which increases the risk of chromosome breaks $[25,95,144]$. Mutations in shmt1 are reported to be associated with genome instability, altered gene expression, and an increased risk for certain types of cancer [95].

Stover and colleagues proposed that mammalian SHMT1, which is translocated into the nucleus upon SUMOylation, has a scaffolding function for the thymidylate synthesis pathway inside the nucleus, which is more important than its actual enzymatic function $[10,53]$. We asked if Drosophila SHMT could function in a similar way in the early embryo and tested this assumption in our mutant system by introducing alternative rescue constructs, which either carried an shmt with an abolished enzyme function or with a different ternary structure (Fig. 24). The non-enzymatic shmt was cloned by a site-specific mutation of a residue, which is known to knock out all enzymatic activities without changes in the ternary structure (Fig. 25) [152]. To design the structure variant we took advantage from an evolutionary switch in the oligomeric state of eucaryotic compared to procaryotic SHMT proteins. It is proposed that the procaryotic SHMT functions as a dimer, whereas eukaryotes have tetrameric SHMT enzymes [23]. 
However, the structure of SHMT monomers is conserved between procaryotic and eucaryotic species. The rescue construct, which replaced the shmt coding sequence with the $E$. coli one, was cloned in a way that the 3'UTR was kept for all three transcripts allowing time- and tissue-specific expression as well as subcellular localisation of the gene products similar to the Drosophila gene (Fig. 24B). Introduction of the bacterial construct into the mutant background rescued the blastoderm phenotype of both X238 and M281R alleles (Table 8), which indicated that the involvement of SHMT in blastoderm development of Drosophila is not due to a structure-mediated scaffolding function. Contrary to this, the catalytically inactive construct was only able to rescue the M281R blastoderm phenotype. The M281R allele contains a single point mutation leading to an amino acid change of methionine into arginine at position 281 [177]. This mutation abolishes the enzymatic function but the amount of SHMT protein in the embryo was only slightly reduced compared to WT and X238, which is probably less stable due to its $\mathrm{C}$ terminal truncation (Fig. 41). The rescue of M281R phenotype by the catalytical inactive shmt transgene indicated an intergenic complementation between these mutant forms of shmt. In summary, the rescue experiments ruled out that the enzymatic activity of SHMT was crucial for blastoderm development in Drosophila, which led to the assumption that the observed blastoderm defect in X238 is either due to disturbed thymidylate or SAM biosynthesis. The contradiction to the published model by the Stover lab could be explained by the fact that mammals have two genes encoding shmt, instead of the one gene in Drosophila. The experiments describing the scaffold function of mammalian SHMT were performed in shmt1/- mice, which still have a functional shmt2 gene [53]. It has been shown previously that SHMT2 is responsible for the residual activity of the thymidylate pathway in shmt1/- mice [9], which makes it likely that the folates supporting the nuclear thymidylate pathway in shmt $1^{-/}$mice are provided by SHMT2. Experiments with mouse cell lines, which are not able to perform de novo thymidylate synthesis revealed that these cells need externally provided thymidylate for mitotic activity and cell survival [75], which would indicate an essential function of shmt for cell survival.

SAM is an important cofactor for cellular methylation reactions [74]. It has been reported that histone methylation at H3K9 is affected upon SAM depletion [158], which is important for heterochromatin formation [125]. S phase lengthening during the syncytial cycles seems to be due to a progressive establishment of late replication of satellite sequences $[135,180]$. Although the observation that X238 mutant embryos developed replication stress in cycle 13 argued against a lack of SAM as the reason of the blastoderm defect, we can not exclude this possibility at the moment (Fig. 39). Further experiments are needed to rule out the involvement of SAM, such as either the measurement of SAM in a metabolomics approach or indirect by analysis of the histone methylation status of H3K9 in cycle 13 of X238 embryos with immunostaining and Western blot.

A second hypothesis is that the defective thymidylate synthesis leads to a reduced dTTP pool, which causes the cell cycle stop in cycle 13 in X238. The maternal supply of dTTP 
or its precursors such as mTHF or dTMP could become limiting in cycle 13. Our observation that X238 could progress into cycle 14 under defined circumstances showed that this limitation does not occur. Interference with a functional Chk1 checkpoint by generation of homozygous grapes mutant X238 germ line clones facilitated progression into mitosis 13 (Fig. 33). A similar effect was observed by inhibition of transcription with $\alpha$-amanitin in X238 embryos (Fig. 29). Injection of GFP-tagged HP1 protein into early X238 embryos enabled them to progress into cycle 14, to perform cellularisation, gastrulation and initation of germband extension (Fig. 45). We assumed that the follicle cells, which were heterozygous mutant in the germline clones, could provide these metabolites into the oocyte. Our finding that shmt had a cell non-autonomous function (Fig. 15 and 16) indicated that the reaction products of the SHMT or subsequent reactions are transported between cells in a tissue. Zhu and colleagues analysed the role of 3'-phosphoadenosine 5'-phosphosulfate (PAPS) synthetase in the establishment of dorsal-ventral polarity in the embryos. Their observation that dorsalised embryos only occur, when PAPS synthetase activity is depleted both in germline cells and the follicle epithelial cells gives further evidence for a transport mechanism between somatic follicle cells and the germline transporting metabolites of the thymidylate synthesis pathway. [184].

\section{2 shmt depletion may activate the replication checkpoint by development of replication stress}

X238 mutant embryos had an extended S phase 13 compared to WT embryos, which was revealed by EdU incorporation experiments and life imaging of GFP-PCNA expressing embryos (Fig. 34-36 and 38). These experiments showed that X238 embryos accumulated actively replicating DNA at the nuclear periphery. Time-lapse recordings of RpA70-eGFP expressing embryos revealed the formation of signal intense foci during cycle 13 of X238 embryos, which persisted until late defect stage (Fig. 39). RpA70 is a member of the RpA complex, which binds to single-stranded DNA during DNA replication, damage and repair [185]. Accumulation of RpA70-eGFP foci during S phase is a marker for persisting areas of single-stranded DNA, a hallmark of replication stress. The RpA complex is known to recruit the ATR kinase [28]. Inhibition of transcription by injection of $\alpha$-amanitin allowed X238 embryos to enter mitosis 13, which confirmed the assumption of a replication stress-mediated cell cycle stop. Further insight into how transcription interfered with replication in X238 embryos could give the investigation of double mutants of X238 and zelda. Zelda is a transcription factor, which is necessary for the activation of early transcribed genes [90]. Mutation of zelda reduces but not eliminates transcriptional activity in the embryo [151], which could be sufficient to finish S phase 13 of X238 and to allow the progression into mitosis. We could rule out overreplication of DNA by staining with the centromeric marker CID, implicating that 
replication stress leads to a slow down or stalling of replication forks at the nuclear periphery (Fig. 37).

The Drosophila checkpoint kinase Grapes (Chk1) is required for early embryonic development. grapes mutants fail to extend their cell cycle length in the syncytial blastoderm cycles, do not stop the cell cycle progression in cycle 14 and develop a mitotic catastrophe phenotype $[56,57]$. Double mutants of X238 and grapes were partially able to enter mitosis 13, which indicated a partial dependence of the X238 cell cycle phenotype on the Grapes mediated DNA checkpoint (Table 13). It is assumed that in the fast cleavage cycles of Drosophila damaged nuclei are eliminated from the embryonic cortex instead of the application of time-consuming DNA repair mechanisms [150, 153]. Although Drosophila mutants of chk2 (mnk) are viable and fertile, Mnk is known to be involved in this nuclear fallout of damaged nuclei [76]. Before actual leaving the cortex, these nuclei are marked by the retainment of specific mRNAs in their interior (e.g kuk and slam), which could be observed in X238 mutants (Fig. 30-32). These data allowed the conclusion that depletion of shmt leads to stalling of the replication complex, which induces replication stress and activates the replication checkpoint, which in turn stops cell cycle progression (Fig. 58A and B). We were only able to indirectly show the activation of checkpoint kinases. A direct measurement of checkpoint activation by Western blot of precisely staged embryos with antibodies specific for activated (phosphorylated) Chk1 and Chk2 and immunostainings of embryos with these antibodies could provide direct evidence for the activation of these kinases. [166, 113]. Further investigation of the checkpoint involvement in the X238 blastoderm defect could be carried out with triple mutants of X238, grapes, and mnk. Depletion of mnk in the grapes background was shown to rescue the mitotic and cellularisation phenotypes of grapes, but not the function of the replication checkpoint [154]. It would be interesting to know, if the depletion of both checkpoint kinases could rescue cell cycle progression and cellularisation of X238, which would prove that the activated replication checkpoint is the actual cause of the premature cell cycle stop.

\subsection{Nuclear SHMT may function in local thymidylate supply at the replication fork}

Our data implicated that a fraction of SHMT was localised in the nucleus (Fig. 44), which is consistent to observations in mammalian cell culture systems made by Stover and coworkers [7, 171]. They identified that all enzymes of the thymidylate pathway (SHMT1, SHMT2 $\alpha$, DHFR, TYMS) are translocated to the nucleus upon SUMOylation, which occurs in $\mathrm{S}$ phase and as a response to DNA damage [7, 9, 59, 171]. Furthermore they revealed the colocalisation of the thymidylate pathway with Lamin B1, which is dependent on SHMT [10]. Moreover, they showed the interaction of SHMT1 with PCNA 
as well as other proteins involved in DNA replication and repair and its enrichment at sites of replication initiation and elongation [10,171]. Stover et al. proposed that the de novo thymidylate synthesis pathway is localised to replication forks and supports processive incorporation of dTTP into DNA during replication [74, 171]. This $\mathrm{S}$ phase specific compartmentalisation could separate the thymidylate pathway from other pathways competing for folate-activated one-carbon units (e.g. purine and SAM biosynthesis pathways) [7, 131, 148]. The enzymes for pathways, which require folate cofactors, are often highly concentrated in the cell and exceed the total cellular folate concentration. So compartmentalisation is an efficient mechanism to support a specific pathway, which is for example more important during a specific phase of the cell cycle (e.g. the thymidylate pathway supporting dTTP synthesis in S phase). This compartmentalisation is often supported by substrate channeling, a mechanism of multienzyme complexes and multifunctional enzymes, which transfer reaction products directly between the active sites of consecutive enzymes or subunits. The reaction products are not released into the medium. This mechanism effectively avoids side reactions as well as diffusion of these reaction products, which results in an increase of reaction velocity and protection of instable intermediate reaction products [85]. Substrate channeling is also a possible mechanism for the thymidylate pathway. All three enzymes (SHMT, DHFR, and TYMS) form a complex, which is dependent on SHMT [9]. It was shown that human cytosolic SHMT and TYMS physically interact with each other, which could allow substrate channeling [171]. In several species (e.g. Plasmodium falciparum), DHFR and TYMS evolved to the bifunctional enzyme Dihydrofolate reductase-thymidylate synthase, which performs substrate channeling for DHF [116]. dUMP and 5,10-mTHF are transformed into thymidylate and DHF by TYMS. DHF is remethylated into THF by DHFR. Since we discovered a nuclear localisation of a subfraction of SHMT in Drosophila syncytial embryos, we assume that also in Drosophila a compartmentalisation mechanism similar to mammalian cells could be established. We hypothesize that SHMT is similarly accumulated in the nuclei of syncytial Drosophila embryos during S phase as in mammals. To test this hypothesis, DNA replication could be blocked in S2 cells and the localisation of SHMT could be analysed by immunostaining subsequently. To rule out, if the missing nuclear fraction of SHMT is responsible for the X238 phenotype, an alternative rescue construct with a modified shmt gene could be cloned, which is not able to be translocated to the nuclei. If SHMT function in the nucleus is necessary, the X238 phenotype should not be rescued.

This compartmentalisation model of the thymidylate pathway is in agreement with our observations, although neither the cell-cycle dependent SUMOylation and translocation to the nucleus nor the accumulation of the pathway at the nuclear lamina are proven in early Drosophila embryos. Drosophila SHMT contains a putative SUMOylation site predicted by "GPS-SUMO", a prediction tool for SUMOylation sites and SUMO-interaction motifs $[122,182]$. This indicates a nuclear import mechanism similar to mammalian SHMT. SUMOylation of Drosophila SHMT could be investigated with an in vitro assay 
using purified SHMT protein and commercial available SUMOylation components and subsequent Western blot analysis with SHMT- and SUMO-specific antibodies [7]. SUMO modification increases the protein size for about $20 \mathrm{kDa}$. Cell cycle specific SUMOylation could be tested by coimmunoprecipation of purified nuclei from staged Drosophila S2 cells with a SUMO-specific antibody and Western blot with an antibody against SHMT [171]. The association with the nuclear lamina could be revealed by coimmunoprecipitation of isolated nuclei of staged embryos with antibodies against lamins and lamin-associated proteins or by further immunostaining experiments in extracted nuclei of Drosophila S2 cells.

Recent work of Vastag and coworkers implicated an involvement of maternally provided dNTP pools in timing of MBT in Xenopus embryos [163]. They performed metabolomic analysis with a mass spectrometry (liquid chromatography-tandem mass spectrometry (LC-MS/MS)) method to investigate the dNTPs pools in the embryo at MBT. They could show that especially the dATP is reduced at MBT. They concluded that the maternally provided dNTP pool is functionally at MBT and that this could be involved in timing of MBT, which is in agreement with previous reports [107, 86]. This would introduce a new mechanism in the complex regulation of timing of the MBT. Our data confirm this hypothesis partially. The reduced SHMT protein amount in X238 mutants could lead to a defective zygotical dTTP production, which could reach the threshold for activation of MBT already in nuclear cycle 13. This would lead to a stop of the syncytial cell cycle progression, as we could observe in X238 mutant embryos. Other hallmarks of MBT (the activation of zygotic expression, degradation of maternally provided mRNA, localisation of cellularisation markers) were established in shmt mutant embryos, however, we did not observe a premature establishment of these hallmarks. This would contradict the hypothesis of Vastag and co-workers. We assume that activation of MBT is not decided by each of these mechanisms separately, but they rather seem to have a hierarchy of importance for the actual activation of MBT. The shmt mutation could affect dTTP synthesis and thereby reduce the dNTP pool. This reduction would lead to premature slow down of DNA replication and induction of replication stress. This would activate the checkpoint kinases Grapes and Mnk, which would pause the cell cycle prematurely. However, in the time between the beginning of interphase 13 and visible morphological defects no cellularisation, a hallmark of Drosophila MBT, occurred. Furthermore, X238 embryos early in cycle 13 showed a gene expression similar to WT embryos in cycle 13. X238 mutants in late phase defect had a comparable gene expression to cellularising WT embryos. These data suggest that only the syncytial cell cycle stop, not MBT is activated prematurely in X238 embryos.

In cycle 13 of X238 mutant embryos, the DNA became accumulated at the nuclear periphery. If Drosophila SHMT is bound to the nuclear lamina and is involved in the replication complex in a similar way as reported in mammalian cells, its depletion could lead to a stalling of the replication forks bound to the nuclear lamina. However, it is reported that early replication takes place in the interior of the nuclei, and only 
late replication of heterochromatin occurs at the nuclear periphery (reviewed in Gilbert et al., 2010 [64]). This indicates that the mutant could be defective in replication of heterochromatic late replicating sequences. Consistent with this, increasing the concentration of the heterochromatin binding protein HP1 by injection allowed X238 embryos to continue cell cycle progression and to finish cellularisation (Fig. 46). Furthermore, immunostaining of endogenous HP1 revealed that its localisation was affected in X238 embryos. HP1 becomes restricted to the apical heterochromatin in cycle 14 WT embryos. This restricted localisation was not observed in X238 embryos, but was rather absent in several nuclei (Fig. 47). This mislocalisation of HP1 could have an effect on the formation and / or maintenance of heterochromatin and contribute to the defective DNA replication. Contradictory to this assumption is the observation that HP1 binding is not necessary for the replication timing in syncytial embryos. Shermoen et al. injected GFP-HP1 into early embryo and followed its distribution and dynamics in life imaging. They could show that although HP1 is present in early syncytial blastoderm cycles, it starts to accumulate at satellite sequences in late cycle 14, after the onset of late replication and heterochromatin specification of these satellite sequences [135]. Beside the well-characterised functions of HP1 in heterochromatin establishment and maintenance, replication timing, and transcriptional control of heterochromatic genes (reviewed in Eissenberg \& Elgin, 2014 [50]), numerous reports indicate a role of HP1 in DNA repair and replication (reviewed in Dinant, 2009 [43]). Recent experiments revealed that HP1 and PCNA form a stable complex involved in DNA replication and DNA repair [159, 160]. This complex accumulates in replication foci and is recruited to sites of DNA damage. Taken this role of HP1 in DNA replication into account, we assume that addition of HP1 in the X238 mutant background could lead to increased recruitment of HP1 to the replication forks. Either by modulating the chromatin structure, spherical interference or by binding to other proteins, transcription could be prevented or reduced at these sites. Thereby HP1 could reduce replication stress and prevent the DNA replication checkpoint-mediated cell cycle stop (Fig. 58C and D). Investigation of histone modifications or localisation of histone modifying enzymes in X238 embryos as well as Nanostring analysis of early transcribed genes in injected and non-injected X238 embryos could provide further insight into the mechanism, with which injected GFP-HP1 rescued the replication defect of shmt mutants. An alternative mechanism of the HP1 rescue could be that introduction of a high-concentrated amount of HP1 induces premature binding to late-replicating sequences and thereby remodels the genome. However, no effect on S phase progression was observed upon injection of GFP-HP1 into WT embryos, which makes this assumption less favourable. Further insight in the replication timing in WT and X238 embryos could be gained by injection of TALE-light proteins followed by time-lapse recordings. These proteins recognise specific satellite sequences and allow live imaging of their replication [180]. A third conceivable mechanism involves an advanced or altered DNA repair, which would allow the fast overcome of missing dTTP by incorporation of dUTP. Due to reports that increased uracil content in the DNA induces genomic instability, leading to chromosome breaks $[25,95,144]$ and to the 
observation that no mitotic defects occurred in the subsequent mitosis 13, we assumed that HP1-injected X238 embryos do not elevate chromosome breaks and so maybe also not incorporate dUTP. To rule out the incorporation of dUTP into the DNA in S phase 13 of X238 embryos a glycosylase assay in isolated nuclei of staged embryos could be carried out, which is a radioactive assay to measure the transformation of uracil into thymine nucleotides [96].

The results obtained in this thesis led to the conclusion, that localised thymidylate synthesis has a direct effect on DNA replication. In X238 embryos, which were defective in de novo nuclear thymidylate synthesis, this could lead to slowing or stalling of replication forks at the nuclear periphery, which could interfere with transcriptional activity. This could activate the DNA replication checkpoint, which could stop the cell cycle progression and prevent entry into mitosis and cellularisation (Fig. 58). 
A

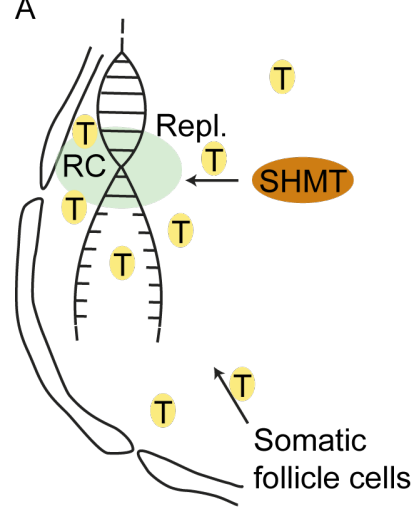

B

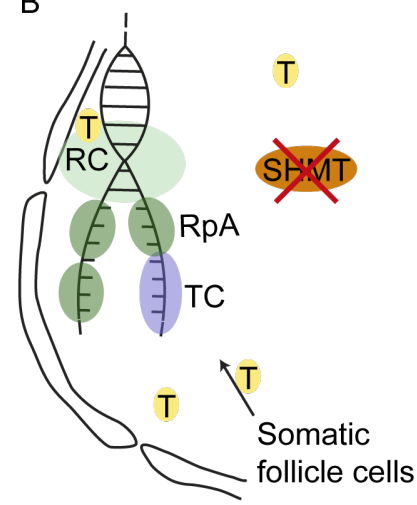

C

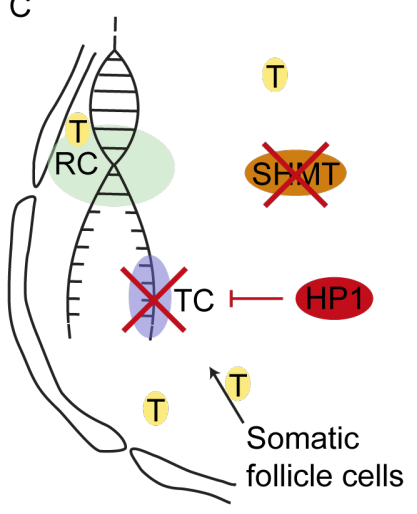

D

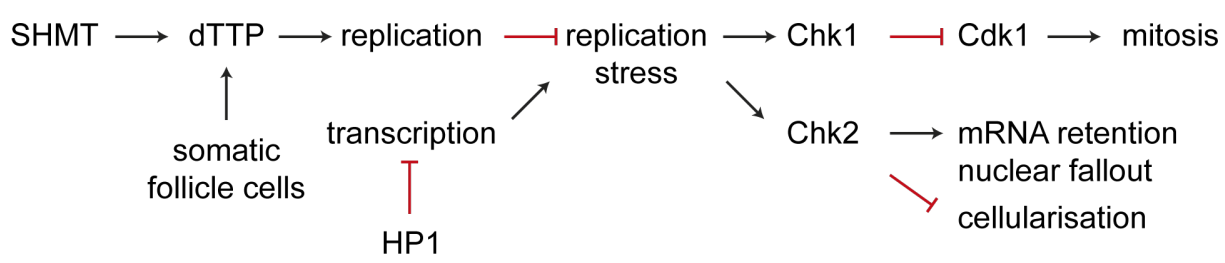

Figure 58: Model for the role of nuclear SHMT in local thymidylate synthesis for DNA replication. (A) Nuclear SHMT recruits the thymidylate synthesis pathway to the replication fork. Local thymidylate synthesis supports fast DNA replication. Additionally, thymine nucleotides or its precursors are provided by somatic follicle cells. (B) Upon SHMT depletion the concentration of thymidylate at the replication fork is reduced, slowing down DNA replication. Increasing transcription in cycle 13 interferes with the slower replication, which leads to replication stress. Persisting areas of single-stranded DNA recruits the binding of the RpA complex, which activates Chk2. Chk2 prevents cellularisation and promotes the nuclear retention of specific mRNAs and nuclear fallout to eliminate damaged nuclei. Furthermore, replication stress activates Chk1, which inhibits Cdk1 thereby preventing the entry into mitosis. (C) Injection of HP1 into SHMT depleted embryos may lead to the local inhibition of transcription near the replication fork, thereby replication stress is reduced and activation of the replication checkpoint is prevented. (D) Scheme for the role of nuclear SHMT in supporting DNA replication. RC: Replication complex, TC: Transcription complex, RpA: Replication protein A complex, SHMT: Serine hydroxymethyltransferase, HP1: Heterochromatin protein 1, Chk1: Checkpoint kinase 1 (Grapes in Drosophila), Chk2: Checkpoint kinase 2 (Mnk in Drosophila), Cdk1: Cyclin dependent kinase 1, T: Thymidylate/Thymine nucleotides. 



\section{Materials and Methods}

\subsection{Materials}

\subsubsection{Reagents}

Unless otherwise stated, all standard chemicals were purchased from the following companies: AppliChem GmbH (Darmstadt, Germany), Gibco BRL (Eggenstein, Germany), Invitrogen (Carlsbad, USA), Merck (Darmstadt, Germany), Roth (Karlsruhe, Germany), Sigma-Aldrich (St. Louis, USA), Thermo Fischer (Braunschweig, Germany).

\subsubsection{Commercial kits}

Following kits were used, according to manufacturer's instructions.

MiniElute Gel extraction Kit

Plasmid Midi Kit Nucleobond AX

In-fusion HD Cloning Kit

Individual Cy3 tyramide reagent pack

Click-IT EdU Alexa Fluor 555 Imaging Kit
(Qiagen, Hilden, Germany)

(Macherey-Nagel, Düren, Germany)

(ClonTech, Mountain View, USA)

(PerkinElmer, Waltham, USA)

(Invitrogen, Carlsbad, USA)

\subsubsection{Enzymes}

Restriction enzymes were purchased from Fermentas (Waltham, USA) or New England Biolabs (Ipswich, USA) and used according to manufacturer's instructions. The following non-restriction enzymes were used:

$\begin{array}{ll}\text { Taq polymerase } & \text { (expressed and purified in the lab) } \\ \text { Pfu polymerase } & \text { (expressed and purified in the lab) } \\ \text { Phusion DNA polymerase } & \text { (Life Technologies, Carlsbad, USA) } \\ \text { T7 RNA polymerase } & \text { (expressed and purified in the lab) } \\ \text { Transcriptor reverse transcriptase } & \text { (Roche, Basel, Switzerland) } \\ \text { DNaseI } & \text { (Roche, Basel, Switzerland) } \\ \text { Proteinase K } & \text { (Roche, Basel, Switzerland) } \\ \text { T4 DNA Ligase } & \text { (Fermentas, Waltham, USA) } \\ \text { Lysozyme } & \text { (AppliChem GmbH, Darmstadt, Germany) }\end{array}$




\subsubsection{Microscopy}

Spinning Disk microscope:

Cameras:

Optics:

Lasers:

Objectives:
Zeiss Observer.Z1, CSU-X1 (Carl Zeiss, Jena, Germany, 2009)

AxioCam MRm

AxioCam ICc1

emCCD evolve 512

Epifluorescence, DIC

$488 \mathrm{~nm}(20 \mathrm{~mW}$, OPSL $)$

$561 \mathrm{~nm}(20 \mathrm{~mW}$, DPSS $)$

A. Plan (10x, NA 0.25)

Plan-Apochromat (25x, NA 0.5,

multi-immersion, DIC)

Plan-Neofluar (40x, NA 1.3, oil, DIC)

LCIPlan-Neofluar (63x, NA 1.3,

multi-immersion, DIC)

Plan-Apochromat (100x, NA 1.4, oil, DIC)

Confocal microscope LSM780: Zeiss AxioObserver.Z1 (Carl Zeiss, Jena, Germany, 2011)

Detection:

PMT

GASP

Lasers:

cooled PMT

Diodelaser ( $405 \mathrm{~nm}, 30 \mathrm{~mW}$ )

Argonlaser $(458 \mathrm{~nm}, 488 \mathrm{~nm}, 514 \mathrm{~nm}, 25 \mathrm{~mW})$

DPSS-Laser $(561 \mathrm{~nm}, 20 \mathrm{~mW})$

HeNe-Laser (633 nm, $5 \mathrm{~mW}$ )

Objectives:

EC Plan-Neofluar (10x, NA 0.3)

LCI Plan-Neofluar (25x, NA 0.8, multi-immersion, DIC)

C-Apochromat (40x, NA 1.2, water)

Plan-Apochromat (63x, NA 1.4, oil)

LCI Plan-Neofluar (63x, NA 1.3, multi-immersion)

Microinjection microscopes:

Leica MZ125

Microinjection microscope

(Leica, Wetzlar, Germany)

(Carl Zeiss, Jena, Germany)

Stereomicroscopes:

Zeiss Stemi 2000

(Carl Zeiss, Jena, Germany) 


\subsubsection{Chromatography}

HisTrap HP columns (GE Healthcare Life Sciences, Little Chalfont, UK)

PD-10 desalting columns (GE Healthcare Life Sciences, Little Chalfont, UK)

\subsubsection{Media}

Media were prepared by lab technicians and student assistants according to standard protocols [128].

\subsubsection{Buffers and solutions}

Unless otherwise stated, all buffers and solutions were prepared according to the protocols in Sambrook and Russel, 2001 [128].

Table 20: Buffers used in the study.

\begin{tabular}{|c|c|c|}
\hline Name & \multicolumn{2}{|c|}{ Composition } \\
\hline \multicolumn{3}{|c|}{ Buffers and solutions for immunostainings and western blot } \\
\hline \multirow[t]{4}{*}{ PBS } & $130 \mathrm{mM}$ & $\mathrm{NaCl}$ \\
\hline & $7 \mathrm{mM}$ & $\mathrm{Na}_{2} \mathrm{PO}_{4}$ \\
\hline & $3 \mathrm{mM}$ & $\mathrm{NaH}_{2} \mathrm{PO}_{4}$ \\
\hline & $\mathrm{pH}$ & 7.4 \\
\hline \multirow[t]{2}{*}{ PBT } & $1 \mathrm{x}$ & PBS \\
\hline & $0.1 \%$ & Tween 20 \\
\hline \multirow[t]{3}{*}{ Fixation solution for embryos } & $4.5 \mathrm{ml}$ & $1 \mathrm{x}$ PBS \\
\hline & $5 \mathrm{ml}$ & Heptane \\
\hline & $0.5 \mathrm{ml}$ or $1 \mathrm{ml}$ & $37 \%$ Formaldehyde \\
\hline \multirow[t]{2}{*}{ Fixation solution for heat fixation } & $0.4 \%$ & $\mathrm{NaCl}$ \\
\hline & $0.03 \%$ & Triton X-100 \\
\hline \multirow[t]{2}{*}{ Fixation solution for imaginal discs } & $9.5 \mathrm{ml}$ & $1 \mathrm{x}$ PBS \\
\hline & $0.5 \mathrm{ml}$ & $37 \%$ Paraformaldehyde \\
\hline \multirow[t]{2}{*}{ Fixation for ovaries } & $9.5 \mathrm{ml}$ & $1 \mathrm{x}$ PBS \\
\hline & $0.5 \mathrm{ml}$ & $37 \%$ Formaldehyde \\
\hline \multirow[t]{2}{*}{ Immunostaining blocking buffer } & $1 \mathrm{x}$ & PBS \\
\hline & $5 \%$ & BSA \\
\hline
\end{tabular}


Buffers used in the study - continuation.

\begin{tabular}{|c|c|c|}
\hline Name & & mposition \\
\hline Imaginal discs washing buffer & $\begin{array}{r}1 \mathrm{x} \\
0.3 \%\end{array}$ & $\begin{array}{l}\text { PBS } \\
\text { Triton X-100 }\end{array}$ \\
\hline Imaginal discs blocking buffer & $\begin{array}{r}1 \mathrm{x} \\
0.3 \% \\
5 \%\end{array}$ & $\begin{array}{l}\text { PBS } \\
\text { Triton X-100 } \\
\text { BSA }\end{array}$ \\
\hline Western blot transfer buffer & $\begin{array}{r}25 \mathrm{mM} \\
175 \mathrm{mM} \\
20 \%\end{array}$ & $\begin{array}{l}\text { Tris } \\
\text { Glycine } \\
\text { Methanol }\end{array}$ \\
\hline Western blot blocking buffer & $\begin{array}{r}1 \mathrm{x} \\
5 \%\end{array}$ & $\begin{array}{l}\text { PBS } \\
\text { milk powder }\end{array}$ \\
\hline Buffers and solutions for Miniprep of plasmi & DNA & \\
\hline Solution I & $\begin{array}{l}50 \mathrm{mM} \\
10 \mathrm{mM}\end{array}$ & $\begin{array}{l}\text { Tris/HCl, pH } 8.0 \\
\text { EDTA }\end{array}$ \\
\hline Solution II & $\begin{array}{r}0.2 \mathrm{mM} \\
1 \%\end{array}$ & $\begin{array}{l}\mathrm{NaOH} \\
\mathrm{SDS}\end{array}$ \\
\hline Solution III & $3 \mathrm{M}$ & Potassium acetate $\mathrm{pH} 5.4$ \\
\hline $\begin{array}{l}\text { Buffers for purification of GST-SHMT-His } \\
\text { Lysis buffer }\end{array}$ & $\begin{array}{r}20 \mathrm{mM} \\
500 \mathrm{mM} \\
20 \mathrm{mM}\end{array}$ & $\begin{array}{l}\mathrm{NaH}_{2} \mathrm{PO}_{4}, \mathrm{pH} 8.0 \\
\mathrm{NaCl} \\
\text { Imidazole }\end{array}$ \\
\hline Washing buffer & $\begin{array}{r}20 \mathrm{mM} \\
500 \mathrm{mM} \\
40 \mathrm{mM}\end{array}$ & $\begin{array}{l}\mathrm{NaH}_{2} \mathrm{PO}_{4}, \mathrm{pH} 8.0 \\
\mathrm{NaCl} \\
\text { Imidazole }\end{array}$ \\
\hline Elution buffer & $\begin{array}{r}20 \mathrm{mM} \\
500 \mathrm{mM} \\
250 \mathrm{mM}\end{array}$ & $\begin{array}{l}\mathrm{NaH}_{2} \mathrm{PO}_{4}, \mathrm{pH} 8.0 \\
\mathrm{NaCl} \\
\text { Imidazole }\end{array}$ \\
\hline
\end{tabular}

Buffers for purification of His-GFP-HP1 and GFP

Lysis buffer

Washing buffer
$50 \mathrm{mM} \quad \mathrm{NaH}_{2} \mathrm{PO}_{4}, \mathrm{pH} 8.0$

$300 \mathrm{mM} \quad \mathrm{NaCl}$

$10 \mathrm{mM}$ Imidazole

$50 \mathrm{mM} \quad \mathrm{NaH}_{2} \mathrm{PO}_{4}, \mathrm{pH} 8.0$

$300 \mathrm{mM} \quad \mathrm{NaCl}$

$20 \mathrm{mM}$ Imidazole 
Buffers used in the study - continuation.

\begin{tabular}{lrl}
\hline Name & \multicolumn{2}{c}{ Composition } \\
\hline Elution buffer & $50 \mathrm{mM}$ & $\mathrm{NaH}_{2} \mathrm{PO}_{4}, \mathrm{pH} 8.0$ \\
& $300 \mathrm{mM}$ & $\mathrm{NaCl}$ \\
$250 \mathrm{mM}$ & Imidazole \\
& $40 \mathrm{mM}$ & HEPES, pH 7.4 \\
Storage buffer & $150 \mathrm{mM}$ & KCl \\
\hline Buffers and solutions for in situ hybridisation & & \\
NTP+Dig labelling mix (10x) & $10 \mathrm{mM}$ & ATP \\
& $10 \mathrm{mM}$ & GTP \\
& $10 \mathrm{mM}$ & CTP \\
& $6.5 \mathrm{mM}$ & UTP \\
$3.5 \mathrm{mM}$ & Dig-11-UTP, pH 7.5 \\
& $50 \%$ & Formamide \\
$5 \mathrm{x}$ & SSC \\
Hybridisation solution & $0.2 \%$ & Tween \\
& $100 \mu \mathrm{g} / \mathrm{ml}$ & tRNA \\
& DEPC- $\mathrm{H}_{2} \mathrm{O}$ & fill up to $50 \mathrm{ml}$ \\
& stored at $-20{ }^{\circ} \mathrm{C}$ & \\
\hline
\end{tabular}

\subsubsection{Plasmid constructs}

Table 21: Plasmids used in the study.

\begin{tabular}{|c|c|c|}
\hline Name & Description & Source \\
\hline pET28a-His-GFP-HP1 & N-terminal His-tag fused to GFP and HP1 & $\begin{array}{ll}\text { P. O'Farrell } \\
{[135]}\end{array}$ \\
\hline pQE-His10-GFPlinker & $\begin{array}{l}\text { N-terminal His-tag fused to GFP with a C- } \\
\text { terminal linker sequence }\end{array}$ & J. Großhans \\
\hline pGEX-6OH & GST-His fusion protein & J. Großhans \\
\hline $\mathrm{pKS}$ & $\begin{array}{l}\text { plasmid used as transient vector for subcloning } \\
\text { steps }\end{array}$ & J. Großhans \\
\hline Kuk cDNA plasmid & plasmid containing the KUK cDNA & $\begin{array}{l}\text { M. Kriebel } \\
\text { (Grosshans } \\
\text { lab) }\end{array}$ \\
\hline Slam cDNA plasmid & plasmid containing the Slam cDNA & $\begin{array}{l}\text { S. Yan } \\
\text { (Grosshans } \\
\text { lab) }\end{array}$ \\
\hline
\end{tabular}


Plasmids used in the study - continuation.

\begin{tabular}{|c|c|c|}
\hline Name & Description & Source \\
\hline $\begin{array}{l}\text { Plasmids generated i } \\
\text { pattB }\end{array}$ & $\begin{array}{l}\text { is study: } \\
\text { plasmid to make transgenic flies with } \\
\text { attB } / \text { phi-C31 system }\end{array}$ & - \\
\hline pGEX-SHMT-His & $\begin{array}{l}\text { SHMT CDS fused with N-terminal GST- and } \\
\text { C-terminal His-tag }\end{array}$ & - \\
\hline pKS-fr1 & $\begin{array}{l}\text { fragment } 1 \text {, subcloning step to make pattB- } \\
\text { SHMT-rescue }\end{array}$ & - \\
\hline pKS-fr2 & $\begin{array}{l}\text { fragment 2, subcloning step to make pattB- } \\
\text { SHMT-rescue }\end{array}$ & - \\
\hline pattB-fr2 & subcloning step to make pattB-SHMT-rescue & - \\
\hline pattB-SHMT-rescue & full rescue construct of X238 & - \\
\hline pattB-5C-left & $\begin{array}{l}\text { subcloning step to make vector for alternate } \\
\text { rescue constructs }\end{array}$ & - \\
\hline pattB-5C & vector for alternate rescue constructs & - \\
\hline pattB-5C-new & vector for alternate rescue constructs & - \\
\hline pattB-E130Q & $\begin{array}{l}\text { alternate rescue construct of } \mathrm{X} 238 \text {, contains } \\
S H M T \text { gene mutated at position } \mathrm{E} 130\end{array}$ & - \\
\hline pattB-Bact & $\begin{array}{l}\text { alternate rescue construct of X238, SHMT } \\
\text { coding sequence replaced by glyA }\end{array}$ & - \\
\hline pattB-Bact2 & $\begin{array}{l}\text { alternate rescue construct of } \mathrm{X} 238, \text { glyA re- } \\
\text { places } S H M T \text { coding sequence from second } \\
\text { start codon }\end{array}$ & - \\
\hline pattB- $\Delta$ CR44108 & $\begin{array}{l}\text { alternate rescue construct of X238, CDS of } \\
S H M T \text { replaces gene sequence from second } \\
\text { start codon, deleting nucleotides } 1-39 \text { of } \\
\text { CR } 44108 \text { gene }\end{array}$ & - \\
\hline
\end{tabular}

\subsubsection{Oligonucleotides}

All oligonucleotides were ordered from Eurofins genomics (Ebersberg, Germany).

Table 22: Oligonucleotides used in this study.

\begin{tabular}{lll}
\hline Oligo & Sequence $\left(5^{\prime} \rightarrow 3^{\prime}\right)$ & Description \\
\hline Cloning: & pGEX-SHMT-His & \\
FW1 & CGCCCATGGATCAGCGGGCGCGCT & forward primer, sequencing pKS-fr1 \\
& CTACA & \\
FW2 & CGCAGATCTCAGGGTCTCCAGGCC & reverse primer, sequencing pKS-fr1 \\
& GGGCA & \\
\hline
\end{tabular}


Oligonucleotides used in this study - continuation.

\begin{tabular}{|c|c|c|}
\hline Oligo & Sequence $\left(5^{\prime} \rightarrow 3^{\prime}\right)$ & Description \\
\hline \multicolumn{3}{|c|}{ Cloning: pKS-fr1 } \\
\hline JG314 & CGGCAAAAAAACCTTCTTGTG & reverse primer \\
\hline JG315 & GTCCGCACATATTCAACGATCG & forward primer \\
\hline \multicolumn{3}{|c|}{ Cloning: pKS-fr2 } \\
\hline JG331 & CGAATTGCACACGCAGCAG & forward primer \\
\hline FW6 & CGATGTGGCCGATATCTTCT & reverse primer \\
\hline \multicolumn{3}{|c|}{ Cloning: pattB-5C-left } \\
\hline FW37 & $\begin{array}{l}\text { TATGCTAGCGGATCCGTGACGGTT } \\
\text { GCCAGGTCG }\end{array}$ & forward primer with BamHI site \\
\hline FW38 & $\begin{array}{l}\text { TCTGTTAACGAATTCTTAATTAAT } \\
\text { AGGTGCTGCCTGCTTTGT }\end{array}$ & reverse primer with EcoRI site \\
\hline \multicolumn{3}{|c|}{ Cloning: pattB-5C } \\
\hline FW39 & $\begin{array}{l}\text { AGCAGGCAGCACCTATTAATTAAG } \\
\text { TCTTTGTAACTGTAATAA }\end{array}$ & forward primer with PacI site \\
\hline FW40 & $\begin{array}{l}\text { TCTGTTAACGAATTCAAAACGCGC } \\
\text { GCTTTTTCT }\end{array}$ & reverse primer \\
\hline \multicolumn{3}{|c|}{ Cloning: pattB-5C-new } \\
\hline FW33 & $\begin{array}{l}\text { TACAGTTACAAAGACATGCAGCGG } \\
\text { GCCGCTCT }\end{array}$ & reverse primer \\
\hline FW51 & $\begin{array}{l}\text { AGCAGGCAGCACCTATTAATTAAA } \\
\text { TTCTGTTCGGATTTATATAGTATTG }\end{array}$ & forward primer with PacI site \\
\hline \multicolumn{3}{|c|}{ Cloning: pKS-SHMT } \\
\hline FW29 & $\begin{array}{l}\text { CTGCAGCCCGGGGGACAGGGTCTC } \\
\text { CAGGCCGGG }\end{array}$ & reverse primer \\
\hline FW30 & $\begin{array}{l}\text { TCTAGAACTAGTGGAATGCAGCGG } \\
\text { GCGCGCTCT }\end{array}$ & forward primer \\
\hline \multicolumn{3}{|c|}{ Mutagenesis: pKS-SHMT } \\
\hline FW41 & $\begin{array}{l}\text { CTTGCCGGGATATCCCTGGGAGTA } \\
\text { CTTGTTGGT }\end{array}$ & reverse primer \\
\hline FW42 & $\begin{array}{l}\text { ACCAACAAGTACTCCCAGGGATAT } \\
\text { CCCGGCAAG }\end{array}$ & forward primer \\
\hline \multicolumn{3}{|c|}{ Cloning: pattB-E130Q } \\
\hline FW33 & $\begin{array}{l}\text { TACAGTTACAAAGACATGCAGCGG } \\
\text { GCGCGCTCT }\end{array}$ & reverse primer \\
\hline FW34 & $\begin{array}{l}\text { AGCAGGCAGCACCTACAGGGTCTC } \\
\text { CAGGCCGGG }\end{array}$ & forward primer \\
\hline
\end{tabular}

Cloning: pattB-Bact 
Oligonucleotides used in this study - continuation.

\begin{tabular}{|c|c|c|}
\hline Oligo & Sequence $\left(5^{\prime} \rightarrow 3^{\prime}\right)$ & Description \\
\hline FW27 & $\begin{array}{l}\text { TACAGTTACAAAGACATGTTAAAG } \\
\text { CGTGAAATGAACA }\end{array}$ & reverse primer \\
\hline FW28 & $\begin{array}{l}\text { AGCAGGCAGCACCTATCAGTGCCG } \\
\text { CCGGAAA }\end{array}$ & forward primer \\
\hline \multicolumn{3}{|c|}{ Cloning: pattB-Bact2 } \\
\hline FW52 & $\begin{array}{l}\text { AGCAGGCAGCACCTAGTGCCGCCG } \\
\text { GAAACC }\end{array}$ & forward primer \\
\hline FW53 & $\begin{array}{l}\text { TAAATCCGAACAGAATATGTTAAA } \\
\text { GCGTGAAATGAACATTG }\end{array}$ & reverse primer \\
\hline \multicolumn{3}{|c|}{ Cloning: pattB- $\Delta$ CR44108 } \\
\hline FW56 & $\begin{array}{l}\text { AGCAGGCAGCACCTACAGGGTCTC } \\
\text { CAGGCCGGG }\end{array}$ & forward primer \\
\hline FW57 & $\begin{array}{l}\text { AAATCCGAACAGAATATGGCCGAT } \\
\text { CAGAAACTGCTGC }\end{array}$ & reverse primer \\
\hline \multicolumn{3}{|c|}{ Sequencing primers } \\
\hline JG158 & & pGEX-SHMT-His \\
\hline PL32 & AGAATACATTTAATTTAGAAAATG & $\begin{array}{l}\text { pattB constructs (binds in white } \\
\text { gene) }\end{array}$ \\
\hline $\begin{array}{l}\text { JG226 } \\
\text { (T3) }\end{array}$ & AАTTAACCCTCACTAAAGGG & $\begin{array}{l}\text { pKS-CG3011, -CG3011-E130Q, -fr1, } \\
\text {-fr2 }\end{array}$ \\
\hline $\begin{array}{l}\text { JG107 } \\
(\mathrm{T} 7)\end{array}$ & GTAATACGACTCACTATAG & $\begin{array}{l}\text { pKS-CG3011, -CG3011-E130Q, -fr1, } \\
\text {-fr2 }\end{array}$ \\
\hline FW3 & CCGGCGTGATCTTCTTCC & $\begin{array}{l}\text { pGEX-SHMT-His, -CG3011, } \\
\text {-CG3011-E130Q, pattB- } \Delta \text { CR44108, } \\
\text {-E130Q }\end{array}$ \\
\hline FW4 & CCTTCATGGCTGGCTATCAT & pattB-5C-left, -5C, -5C-new \\
\hline FW7 & CTGCCTGGTCAGCTTTATG & $\begin{array}{l}\text { pKS-CG3011, -CG3011-E130Q, -fr2, } \\
\text { pattB-5C-new, -E130Q }\end{array}$ \\
\hline FW8 & GCAAAAACTGGAAACTGGTG & $\begin{array}{l}\text { pKS-CG3011, -CG3011-E130Q, -fr2, } \\
\text { pattB-5C-new, -E130Q }\end{array}$ \\
\hline FW9 & CTTCATGCGTTTCATTCAG & $\begin{array}{l}\text { pKS-CG3011, -CG3011-E130Q, -fr2, } \\
\text { pattB-5C-new, -E130Q }\end{array}$ \\
\hline FW10 & GGTGTCGAATATTTTGGATG & $\begin{array}{l}\text { pKS-fr2, pattB-5C, -5C-new, -Bact, } \\
\text {-E130Q }\end{array}$ \\
\hline FW13 & CTGAATGAAACGCATGAAG & $\begin{array}{l}\text { pKS-CG3011, -CG3011-E130Q, } \\
\text { pattB-5C-new, -E130Q, }\end{array}$ \\
\hline FW14 & CCGCAAAGTGAGAAGAGTC & pattB-5C \\
\hline FW15 & GAGTGCAACATCTCTCTCCA & pattB-5C \\
\hline FW16 & GATCAACTACCGCCACCT & $\begin{array}{l}\text { pattB constructs (binds in attB } \\
\text { site) }\end{array}$ \\
\hline
\end{tabular}


Oligonucleotides used in this study - continuation.

\begin{tabular}{lll}
\hline Oligo & Sequence $\left(\mathbf{5}^{\prime} \rightarrow \mathbf{3}^{\prime}\right)$ & Description \\
\hline FW17 & CCTCCAGATCGTAGAGTACC & pGEX-SHMT-His, pKS-CG3011, \\
& & -CG3011-E130Q, pattB- $\Delta$ CR44108, \\
& & -E130Q \\
FW18 & CATTAACGCCCCACTTCT & -GEX-SHMT-His, pKS-CG3011, \\
& & -CG3011-E130Q, pattB- $\Delta$ CR44108, \\
& & pKS-CG3011, -CG3011-E130Q, \\
FW19 & TGTAACTTTTCGCAACCATC & pattB-5C-new, -Bact2, - $\Delta$ CR44108, \\
& & -E130Q \\
& & pKS-CG3011, -CG3011-E130Q, \\
FW20 & GGTGATCCTGCTCTAATCCT & pattB-E130Q \\
& & pattB-5C-left \\
FW43 & ATGATAGCCAGCCATGAAGG & pattB-Bact, -Bact2, - $\Delta$ CR44108, \\
FW44 & TCCATTCCAAAAATAAAACTCCA & -E130Q \\
& & pattB-Bact, -Bact2 \\
FW45 & GATGCTGTCAGCGATTTCAC & pattB-Bact, -Bact2 \\
FW46 & TAGTTTTCGGAGGCGATCAG & pattB-Bact, -Bact2 \\
FW48 & AAGGTGGTAGCGAAGAGCTG & pattB-Bact, -Bact2 \\
FW49 & ATCGATCGTGCGAAAGAACT & pattB- $\Delta$ CR44108 \\
FW63 & CTTGAATTCGGGACTCTTG & pattB- $\Delta$ CR44108 \\
FW64 & ATATCGTGCTACTCCCGTCT &
\end{tabular}

\subsubsection{Antibodies}

Table 23: Primary antibodies used for this thesis.

\begin{tabular}{lcccr}
\hline & & \multicolumn{2}{c}{ Dilution / working concentration } & \\
\cline { 3 - 4 } Antibody & Raised in & Staining & Western & Source \\
\hline CID & Rabbit & $1: 500$ & - & S. Heidmann \\
Dia & Rabbit & $1: 1000$ & - & ZMBH \\
Dlg & Mouse & $1: 100(\sim 0.4 \mu \mathrm{g} / \mathrm{ml})$ & - & Hybridoma Bank 4F3 \\
Eve & Guinea pig & $1: 1000$ & - & J. Großhans \\
Frs & Rabbit & $1: 500$ & - & J. Großhans \\
GFP-HP1 & Rabbit & $1: 2500$ & - & \\
Kuk & Rabbit & $1: 10000$ & - & J. Großhans \\
& Guinea Pig & $1: 5000$ & - & J. Großhans \\
Lamin Dm0 & Mouse & $1: 500$ & - & H. Saumweber \\
SHMT & Rabbit & $1: 5000$ & $1: 20000$ & \\
& Guinea Pig & $1: 1000$ & $1: 5000$ & J. Großhans
\end{tabular}


Primary antibodies used for this thesis - continuation.

\begin{tabular}{lcccr}
\hline & & \multicolumn{2}{c}{ Dilution/working concentration } & \\
\cline { 3 - 4 } Antibody & Raised in & Staining & Western & Source \\
\hline \multirow{2}{*}{ Sry- $\alpha$} & Guinea pig & $1: 5000$ & - & J. Großhans \\
Twine & Mouse & $1: 10$ & - & Hybridoma Band 6B12 \\
$\gamma$-Tubulin & Rat & $1: 500$ & - & E. Wieschaus \\
\hline
\end{tabular}

Secondary Alexa-conjugated antibodies were purchased from Life Technologies (Carlsbad, USA) and used at a final concentration of 1:500 $(4 \mu \mathrm{g} / \mathrm{ml})$. DNA staining was performed with DAPI (Sigma-Aldrich, St. Louis, USA) at a working concentration of $0.2 \mu \mathrm{g} / \mathrm{ml}$. GFP-Booster-Atto488 was purchased from Chromotek (Martinsried, Germany) and used at a final concentration of $2 \mu \mathrm{g} / \mathrm{ml}$. Anti-Digoxigenin-peroxidase antibodies, purchased from Roche (Basel, Switzerland), were diluted 1:200 $(0.75 \mathrm{U} / \mathrm{ml})$. Secondary antibodies for western blots, IRDye-800CW and IRDye-680, were purchased from LI-COR Biotechnology (Bad Homburg, Germany) and used at a dilution of 1:20000 $(0.05 \mu \mathrm{g} / \mathrm{ml})$.

\subsubsection{Bacterial strains}

In this study, the $E$. coli strains DH5 $\alpha$ and BL21DE3 were used for plasmid amplification and protein expression, respectively.

\subsubsection{Fly stocks}

Unless otherwise stated, all fly stocks were obtained from Bloomington Stock Center (Bloom.).

Table 24: Stocks used for this thesis.

\begin{tabular}{lrr}
\hline Stock name & Stock genotype & Source \\
\hline OrR & $\frac{+}{+}$ & J. Großhans \\
& w $; \frac{\mathrm{ubi}-\mathrm{ECadGFP}\left\{\mathrm{w}^{+}\right\} \mathrm{His} 2 \mathrm{HvRFP}\left\{\mathrm{w}^{+}\right\}}{\mathrm{CyO}}$ & $\mathrm{J}$. Großhans \\
GFPCad,HisRFP &
\end{tabular}


4.1 Materials

Stocks used for this thesis - continuation

\begin{tabular}{|c|c|c|}
\hline Stock name & Stock genotype & Source \\
\hline X238Frt19A & $\frac{\text { y pn CG3011[X238] sn Frt [19A] }\{\text { neo }\}}{\text { FM7c, y w w } \mathrm{w}^{\mathrm{a}} \mathrm{B}}$ & J. Großhans \\
\hline-- & --------------------------- & 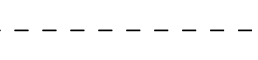 \\
\hline X238Frt18E & $\frac{\text { w CG3011[X238] f FrtX[9-2]hs-Flp }[122]\left\{\mathrm{ry}^{+}\right\}}{\text {FM7c }}$ & J. Großhans \\
\hline M281R & $\frac{\mathrm{y}^{1} \mathrm{w}^{+} \text {CG3011[A] Frt }[19 \mathrm{~A}]\{\mathrm{neo}\}}{\text { FM7c,GFP,sn }}$ & Bloom. \\
\hline---------1 & --------------------------- & -------- \\
\hline$\Delta 15$ & $\mathrm{w} ; \frac{\mathrm{Sp}}{\mathrm{CyO}} ; \mathrm{dur}^{+}\left\{\mathrm{w}^{+}\right\}, \mathrm{Df}(3 \mathrm{R}) \mathrm{kuk} 15, \mathrm{e}, \mathrm{ca}$ & J. Großhans \\
\hline---------- & ----------------------------- & -------- \\
\hline grapes & $\mathrm{y} \mathrm{w} ; \frac{\operatorname{grapes}[1]\left\{\mathrm{w}^{+}\right\}}{\mathrm{CyO}, \mathrm{hid}}$ & {$[137]$} \\
\hline GFP-PCNA & $\frac{\text { UASp-GFP-PCNA }}{\mathrm{CyO}}$ & B. Edgar \\
\hline RpA70eGFP & y w; RpA70eGFP[attP2] $\left\{\mathrm{w}^{+}\right\}$ & E. Wieschaus \\
\hline--------- & ----------------------------- & -------- \\
\hline mat $67 ; 15$ & 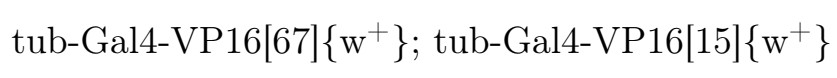 & D. St.Johnson \\
\hline---------- & ---------------------------- & -------- \\
\hline ovo19 & $\frac{\text { ovo[D1-18]hs-Flp,y,w,sn,FrtX[19A] }[\text { neo }\}}{C(1) D X, y, w, f}$ & Bloom. \\
\hline-- & & \\
\hline ovo19; & $\frac{\text { ovo[D1-18]hs-Flp,y,w,sn,FrtX[19A] }[\text { neo }\}}{\mathrm{C}(1) \mathrm{DX}, \mathrm{y}, \mathrm{w}, \mathrm{f}} ;$ & J. Großhans \\
\hline HisRFP,CadGFP & $\frac{\mathrm{ubi}-\mathrm{ECadGFP}\left\{\mathrm{w}^{+}\right\} \operatorname{His} 2 \operatorname{AvRFP}\left\{\mathrm{w}^{+}\right\}}{\mathrm{CyO}}$ & \\
\hline & & \\
\hline ovo18 & $\frac{\mathrm{ovo}[\mathrm{D} 2] \mathrm{v}[24] \mathrm{FrtX}[9-2]\left\{\mathrm{w}^{+}\right\}}{\mathrm{C}(1) \mathrm{DX}, \mathrm{y}, \mathrm{w}, \mathrm{f}}$ & B-1843(Tüb.) \\
\hline ovo18; & $\frac{\text { ovo[D1-18]hs-Flp,y,w,sn,FrtX[19A] }\{\text { neo }\}}{\mathrm{C}(1) \mathrm{DX}, \mathrm{y}, \mathrm{w}, \mathrm{f}} ;$ & J. Großhans \\
\hline
\end{tabular}


Stocks used for this thesis - continuation

\begin{tabular}{|c|c|c|}
\hline Stock name & Stock genotype & Source \\
\hline HisRFP,CadGFP & $\frac{\mathrm{ubi}-\mathrm{ECadGFP}\left\{\mathrm{w}^{+}\right\} \operatorname{His} 2 \operatorname{AvRFP}\left\{\mathrm{w}^{+}\right\}}{\mathrm{CyO}}$ & \\
\hline ovoD2L & y w hs-Flp[122]; $\frac{\text { ovoDFrt2L[40A] }}{\text { If } / \text { CyO, hs-hid }\left\{\mathrm{w}^{+}\right\}}$ & S. Luschnig \\
\hline nlsGFP,Frt18E & $\begin{array}{l}\text { y w ubi-nlsGFP }\left\{\mathrm{w}^{+}\right\}[\mathrm{X} 1] \operatorname{FrtX}[9-2]\left\{\mathrm{w}^{+}\right\} \\
\text {hs-Flp[38] }\left\{\mathrm{ry}^{+}\right\}\end{array}$ & S. Luschnig \\
\hline Frt18E & $\frac{\mathrm{y}^{1} \mathrm{w}, \text { FrtX[9-2]hs-Flp }[122]\left\{\mathrm{ry}^{+}\right\}}{\text {FM7c }}$ & J. Großhans \\
\hline Sas6-GFP & $\mathrm{w} ; \frac{\mathrm{ubi}-\mathrm{GFP}-\mathrm{Sas} 6\left\{\mathrm{w}^{+}\right\}}{\mathrm{TM} 6 \mathrm{c}, \mathrm{Sb}}$ & J. Raff \\
\hline $\begin{array}{l}\text { Sas4-GFP } \\
-\ldots---\ldots-\ldots\end{array}$ & $\mathrm{w} ; \frac{\mathrm{ubi}-\mathrm{GFP}-\mathrm{Sas} 4\left\{\mathrm{w}^{+}\right\}}{\mathrm{CyO}}$ & J. Raff \\
\hline ELMO, & $\mathrm{w} ; \frac{2 \mathrm{~L} 367, \mathrm{al}, \mathrm{dp}, \mathrm{b}, \mathrm{pr}, \operatorname{Frt}[40 \mathrm{~A}]\{\mathrm{neoR}\}}{\mathrm{CyO}} ;$ & Z. Lv \\
\hline Sas6-GFP & $\frac{\mathrm{ubi}-\mathrm{GFP}-\mathrm{Sas} 6\left\{\mathrm{w}^{+}\right\}}{\mathrm{TM} 6 \mathrm{c}, \mathrm{Sb}}$ & \\
\hline Utrophin-GFP & $\mathrm{w} ; \frac{\mathrm{sqh}-\mathrm{Utr}:: \mathrm{GFP}}{\mathrm{CyO}}$ & T. Lecuit \\
\hline
\end{tabular}

Zipper-GFP

$\mathrm{w}^{*} ; \frac{\mathrm{P}\{\mathrm{PTT}-\mathrm{GC}\} \text { zip[CC01626] }}{\mathrm{SM} 6 \mathrm{a}}$

Bloom.

Kin1RNAi

$\mathrm{y}^{1} \mathrm{v}^{1} ;$ PTRiP.JF01939attP2

Bloom.

MTDGal4

P[COG-Gal4VP16; NGT40; nanos-Gal4VP16]

[101]

\section{Generated in this work:}


Stocks used for this thesis - continuation

\begin{tabular}{|c|c|c|}
\hline Stock name & Stock genotype & Source \\
\hline SHMT-rescue & $\mathrm{w} ; \operatorname{attB}[68 \mathrm{~A} 4]\left\{\mathrm{CG} 3011, \mathrm{w}^{+}\right\}$ & - \\
\hline-------- & ---------------- & ---- \\
\hline Bact & w;attB $[68 \mathrm{~A} 4]\left\{{\left.\text { Bact }, \mathrm{w}^{+}\right\}}^{+}\right.$ & - \\
\hline-------- & --------------- & \\
\hline Bact2 & $\mathrm{w} ; \operatorname{attB}[68 \mathrm{~A} 4]\left\{\right.$ Bact $\left.2, \mathrm{w}^{+}\right\}$ & - \\
\hline
\end{tabular}
E130Q
$\mathrm{w} ; \operatorname{attB}[68 \mathrm{~A} 4]\left\{\mathrm{E} 130 \mathrm{Q}, \mathrm{w}^{+}\right\}$

$\Delta \mathrm{CR} 44108$

$\mathrm{w} ; \operatorname{attB}[68 \mathrm{~A} 4]\left\{\Delta \mathrm{CR} 44108, \mathrm{w}^{+}\right\}$

X238-19;

$\frac{\text { y pn CG3011[X238] sn Frt[19A] }\{\text { neo }\}}{{\text { FM7c,y, }{ }^{\mathrm{a}}, \mathrm{sn}, \mathrm{B}}}$;

SHMT-rescue

$\mathrm{w} ; \operatorname{attB}[68 \mathrm{~A} 4]\left\{\mathrm{CG} 3011, \mathrm{w}^{+}\right\}$

X238-19;

$\frac{\text { y pn CG3011[X238] sn Frt[19A] } 2 \text { neo }\}}{{\text { FM7c,y,w }{ }^{\mathrm{a}}, \mathrm{sn}, \mathrm{B}}}$;

Bact2

$\mathrm{w} ; \operatorname{attB}[68 \mathrm{~A} 4]\left\{{\left.\text { Bact } 2, \mathrm{w}^{+}\right\}}^{+}\right.$

X238-18;

$\frac{\text { w CG3011[X238] f FrtX[9-2]hs-Flp[122] }\left\{\mathrm{ry}^{+}\right\}}{\text {FM7c }}$;

SHMT-rescue

$\mathrm{w} ; \operatorname{attB}[68 \mathrm{~A} 4]\left\{\mathrm{CG} 3011, \mathrm{w}^{+}\right\}$

M281R;

$$
\frac{\left.\mathrm{y}^{1} \mathrm{w}^{+} \text {CG3011[A],Frt [19A }\right]\{\text { neo }\}}{\text { FM7c,GFP, } \mathrm{sn}^{+}} ;
$$

SHMT-rescue

$\mathrm{w} ; \operatorname{attB}[68 \mathrm{~A} 4]\left\{\mathrm{CG} 3011, \mathrm{w}^{+}\right\}$

M281R;

$$
\frac{\mathrm{y}^{1} \mathrm{w}^{+} \text {CG3011[A] Frt }[19 \mathrm{~A}]\{\text { neo }\}}{\text { FM7c,GFP, } \mathrm{sn}^{+}} ;
$$

E130Q w;attB[68A4] $\left\{\mathrm{E} 130 \mathrm{Q}, \mathrm{w}^{+}\right\}$

X238-18; grapes $\frac{\left.\mathrm{w} \text { CG3011[X238] f FrtX[9-2]hs-Flp[122] } \text { ry }^{+}\right\}}{\text {FM7c }}$; 
Stocks used for this thesis - continuation

\begin{tabular}{|c|c|c|}
\hline Stock name & Stock genotype & Source \\
\hline & $\frac{\text { grapes }[1]\left\{\mathrm{w}^{+}\right\}}{\mathrm{CyO}, \mathrm{hid}}$ & \\
\hline X238-19; GFP-PCNA & $\begin{array}{c}\text { y pn CG3011[X238] sn Frt[19A]\{neo }\} \\
\text { FM7c,y,wa }, \text { sn,B } \\
\frac{\text { UASp-GFP-PCNA }}{\text { CyO }}\end{array}$ & - \\
\hline ovo19; mat67 & $\begin{array}{c}\frac{\text { ovo[D1-18]hs-Flp,y,w,sn,FrtX }[19 \mathrm{~A}]\{\text { neo }\}}{\mathrm{C}(1) \mathrm{DX}, \mathrm{y}, \mathrm{w}, \mathrm{f}} \\
\frac{\text { tub-Gal4-VP16 }\left\{\mathrm{w}^{+}\right\}[67]}{\mathrm{CyO}}\end{array}$ & \\
\hline ovo19; RpA70eGFP & $\begin{array}{c}\frac{\mathrm{ovo}[\mathrm{D} 1-18] \text { hs-Flp,y,w,sn,FrtX }[19 \mathrm{~A}]\{\mathrm{neo}\}}{\mathrm{C}(1) \mathrm{DX}, \mathrm{y}, \mathrm{w}, \mathrm{f}} \\
\text { y,w;RpA70eGFP }[\mathrm{attP} 2]\left\{\mathrm{w}^{+}\right\}\end{array}$ & - \\
\hline ovo18; grp,mnk & $\begin{array}{c}\frac{\text { ovo[D2] }\left[\mathrm{v}[24] \operatorname{FrtX}[9-2]\left\{\mathrm{w}^{+}\right\}\right.}{\mathrm{C}(1) \mathrm{DX}, \mathrm{y}, \mathrm{w}, \mathrm{f}} \\
\frac{\operatorname{grp}[\mathrm{fs} 1] \mathrm{mnk}[\mathrm{P} 6]}{\mathrm{CyO}}\end{array}$ & - \\
\hline Sas4GFP,Kin1RNAi & $\mathrm{w} ; \frac{\text { ubi-GFP-Sas } 4\left\{\mathrm{w}^{+}\right\}}{\mathrm{CyO}} ; \mathrm{PTRiP.JF01939attP2}$ & - \\
\hline
\end{tabular}




\subsubsection{Other materials}

Pipet-aid

Micropipettes

Micropipette tips

Safe-Seal RNase-free tips

Glass pipettes

Pasteur pipettes

Coverslips

Glass slides

Fly vials

Petri dishes

Falcon tubes

Eppendorf tubes

PCR tubes

Protein concentrator

Minisart Single Use Filter Units $0.22 \mu \mathrm{m}$

Ribolock RNase inhibitor

Cy3-UTP

Complete Mini (EDTA-free) Protease Inhibitor Cocktail

Aquapolymount

$10 \mathrm{~S}$ and 3S VoltaLef Halocarbon oil

H1-Alexa488

$\alpha$-Amanitin

Colcemide

Latrunculin A

Jasplakinolide

Y-27632
(Drummond, Birmingham, USA)

(Gilson, Middleton, USA)

(Sarstedt, Nürnbrecht, Germany)

(Biozym, Wien, Austria)

(Brand, Wertheim, Germany)

(Brand, Wertheim, Germany)

(Thermo Fischer, Braunschweig,

Germany)

(Thermo Fischer, Braunschweig,

Germany)

(Greiner, Kremsmünster, Austria)

(Greiner, Kremsmünster, Austria)

(BD, Heidelberg, Germany)

(Eppendorf, Hamburg, Germany)

(Brand, Wertheim, Germany)

(Sartorius, Göttingen, Germany)

(Sartorius, Göttingen, Germany)

(Thermo Fischer, Braunschweig

, Germany)

(PerkinElmer, Waltham, USA)

(Roche, Basel, Switzerland)

(Polysciences Inc., Warrington, USA)

(Lehmann \& Voss \& Co.,

Hamburg, Germany)

(Life technologies, Carlsbad, USA)

(AppliChem, Darmstadt,

Germany)

(CalbioChem, Darmstadt, Germany)

(Cayman Chemicals, Michigan, USA)

(Sigma-Aldrich, St-Louis, USA)

(Sigma-Aldrich, St-Louis, USA) 


\subsubsection{Other equipment}

Äkta pure

Odyssey CLx Infra-red imaging system

Thermo cycler

P-87 Flaming/Brown Micropipette Puller

EmulsiFlex-C5 Microfluidiser

FemtoJet Microinjector

Semi-Dry Transfer Cell

Concentrator 5301
(GE Healthcare Life Sciences, Little

Chalfont, UK)

(LI-COR Biosciences, Bad Homburg, Germany)

(Bio-Rad, München, Germany)

(Sutter Instrument Co., Novato, USA)

(Avestin, Ottawa, Canada)

(Eppendorf, Hamburg, Germany)

(Bio-Rad, München, Germany)

(Eppendorf, Hamburg, Germany)

\subsubsection{Software}

Zen 2012

ImageJ

Photoshop CS6

Illustrator CS6

Lasergene

Excel

Word

Powerpoint

LaTeX (TexLive \& TeXMaker)

Mendeley Desktop

HHpred

PyMol
(Carl Zeiss, Jena, Germany)

(NIH, Bethesda, USA)

(Adobe, San Jose, USA)

(Adobe, San Jose, USA)

(GATC Biotech, Konstanz, Germany)

(Microsoft, Redmond, USA)

(Microsoft, Redmond, USA)

(Microsoft, Redmond, USA)

(LaTeX Project, Mainz, Germany)

(Mendeley LTD., London, UK)

(University of Tübingen, Tübingen, Germany, http://toolkit.tuebingen.mpg.de/hhpred)

(DeLano Scientific LLC, Portland, USA) 


\subsection{Methods}

All standard procedures, unless otherwise stated, were carried out according to the protocols described in Sambrook \& Russel, 2001 [128] and are not described separately in this chapter.

\subsubsection{DNA and RNA methods}

\section{Polymerase chain reaction}

PCR reactions were carried out using Taq $\left(72^{\circ} \mathrm{C}\right.$ elongation temperature $)$ or $\mathrm{Pfu}\left(68^{\circ} \mathrm{C}\right)$ DNA polymerase. Standard PCR reactions were carried out with the following components:

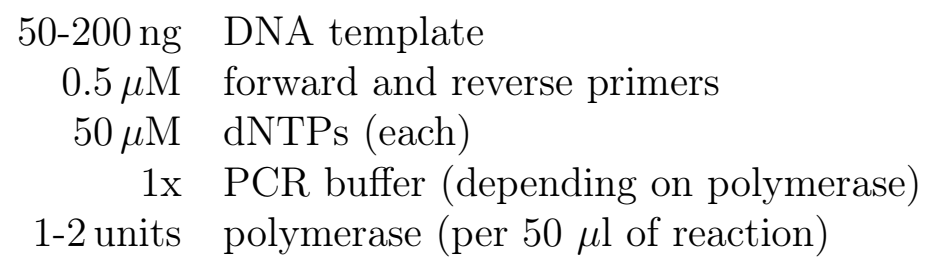

Following conditions were used for standard PCR reactions:

Step 1 (Initial denaturation):

Step 2 (Denaturation):

$\begin{array}{ll}95^{\circ} \mathrm{C} & 3 \mathrm{~min} \\ 95^{\circ} \mathrm{C} & 30 \mathrm{sec}\end{array}$

Step 3 (Annealing):

$50-65^{\circ} \mathrm{C} \quad 30 \mathrm{sec}$

Step 4 (Elongation): $\quad \quad 72 / 68^{\circ} \mathrm{C} \quad 1 \mathrm{~min} / \mathrm{kb}$

depending on primer

Step 5 (Repetition of cycles): Steps 2 to 430 cycles

Step 6 (Final elongation): $\quad 72^{\circ} \mathrm{C} \quad 10 \mathrm{~min}$

Step 7 (Hold): $\quad 12^{\circ} \mathrm{C} \quad \infty$

PCR reactions using Phusion polymerase were carried out with the following conditions:

Step 1 (Initial denaturation): $\quad 98^{\circ} \mathrm{C} \quad 30 \mathrm{sec}$

Step 2 (Denaturation): $\quad 98^{\circ} \mathrm{C} \quad 10 \mathrm{sec}$

Step 3 (Annealing): $\quad 50-70^{\circ} \mathrm{C} \quad 30 \mathrm{sec}$ depending on primer

Step 4 (Elongation): $\quad 72^{\circ} \mathrm{C} \quad 15 \mathrm{sec} / \mathrm{kb}$ depending on amplicon

Step 5 (Repetition of cycles): Steps 2 to 430 cycles

Step 6 (Final elongation): $\quad \quad 72^{\circ} \mathrm{C} \quad 10 \mathrm{~min}$

Step 7 (Hold): $\quad 12{ }^{\circ} \mathrm{C} \quad \infty$ 


\section{Site-directed mutagenesis}

Site-directed mutagenesis was used in order to exchange the 130th aa of SHMT from glutamic acid to glutamine (GAG to CAG). Standard pipetting scheme was used with 10 ng of template, primers FW41 and FW42 and Pfu polymerase. PCR was carried out with the following programme:

$\begin{array}{llrl}\text { Step } 1 \text { (Initial denaturation): } & 95^{\circ} \mathrm{C} & 3 \mathrm{~min} & \\ \text { Step } 2 \text { (Denaturation): } & 95^{\circ} \mathrm{C} & 1 \mathrm{~min} & \\ \text { Step } 3 \text { (Annealing): } & 50-65^{\circ} \mathrm{C} & 1 \mathrm{~min} & \text { (gradient PCR) } \\ \text { Step } 4 \text { (Elongation): } & 68^{\circ} \mathrm{C} & 6.5 \mathrm{~min} & \\ \text { Step } 5 \text { (Repetition of cycles): } & \text { Steps } 2 \text { to } 4 & 20 \text { cycles } & \\ \text { Step 6 (Final elongation): } & 68^{\circ} \mathrm{C} & 10 \mathrm{~min} & \\ \text { Step 7 (Hold): } & 12^{\circ} \mathrm{C} & \infty\end{array}$

\section{In-fusion cloning}

In-fusion cloning procedure was carried out according to the manufacturer's instructions.

\section{Preparation of genomic DNA from bacterial cells}

Genomic DNA was purified from Escherichia coli (E. coli) as described [170].

\section{Sequencing of plasmid constructs and PCR products}

DNA sequencing was carried out by the sequencing facility at the Department of Developmental Biochemistry, GZMB, University of Göttingen.

\section{Cloning of GST-SHMT-His}

PCR to amplify the coding sequence of $S H M T$ was carried out with the yeast-two-hybrid library ovoIb as template, and FW1 and FW2 as primers. Phusion polymerase was used according to manufacturer's instructions (2-step-protocol). PCR was carried out with the following programme: 


$\begin{array}{lcr}\text { Step } 1 \text { (Initial denaturation): } & 98^{\circ} \mathrm{C} & 30 \mathrm{sec} \\ \text { Step 2 (Denaturation): } & 98^{\circ} \mathrm{C} & 10 \mathrm{sec} \\ \text { Step } 3 \text { (Annealing/Elongation): } & 72^{\circ} \mathrm{C} & 50 \mathrm{sec} \\ \text { Step 4 (Repetition of cycles): } & \text { Steps 2 to 3 } & 30 \text { cycles } \\ \text { Step 5 (Final elongation): } & 72^{\circ} \mathrm{C} & 10 \mathrm{~min} \\ \text { Step 6 (Hold): } & 12^{\circ} \mathrm{C} & \infty\end{array}$

Both PCR product and vector (pGEX-6OH) were cut with the restriction enzymes BglII and NcoI. Purification, ligation, transformation, colony-PCR and preparation of plasmid DNA was done according to standard protocols, as for all other cloning procedures.

\section{Cloning of pattB-SHMT-rescue}

The genomic region surrounding the gene CG3011 contained multiple EcoRI and BamHI sites. Therefore cloning of the plasmid pattB-SHMT-rescue was performed in several subcloning steps. First, fragment fr1, which was flanked by two BamHI sites, was cloned into a pKS vector. PCR of fr1 was carried out with Bac5C (a Bac clone containing the $\mathrm{X}$-chromosomal genomic region $5 \mathrm{C}$ ) as template, JG314 and JG315 as primers, and Phusion polymerase $\left(55^{\circ} \mathrm{C}\right.$ annealing temperature $)$. PCR product and vector were cut with BamHI restriction enzyme for ligation.

Second, fragment fr2, flanked by an EcoRI site and the same BamHI site as fr1, was cloned into a pKS vector. For this, primers FW6 and JG331 were used $\left(58^{\circ} \mathrm{C}\right.$ annealing temperature).

After verifying the fragments by sequencing, pKS-fr2 and the vector pattB were cut with EcoRI and BamHI and ligated to pattB-fr2. This plasmid and pKS-fr1 were cut with BamHI and ligated to pattB-SHMT-rescue. Direction of inserted fr1 was determined by restriction enzyme digestion.

\section{Cloning of alternate rescue constructs}

Alternate gene sequences of SHMT were cloned into pattB-SHMT-rescue plasmid, replacing the WT gene sequence between start and stop codon. To achieve this, a plasmid (pattB-5C) was generated, which is similar to pattB-SHMT-rescue, but lacks the coding region of SHMT. Furthermore, a PacI site was inserted at this place, allowing the insertion of alternate coding regions. To achieve this, In-fusion cloning was performed, allowing cloning without the necessity of restriction sites.

First, the sequence of pattB-SHMT-rescue 3' to the coding region was amplified by PCR with primers FW37 and FW38 $\left(54-66{ }^{\circ} \mathrm{C}\right.$ gradient annealing temperatures) and the plasmid pattB-5C-left was generated by inserting into BamHI- and EcoRI-cut pattB. 
pattB-5C was generated by cutting pattB-5C-left with PacI and inserting the genomic region 5' of SHMT. This was performed with FW39 and FW40 as primers $\left(51-63^{\circ} \mathrm{C}\right.$ gradient annealing temperatures).

pKS-SHMT was generated by subcloning the SHMT gene into pKS, which was done with primers FW29 and FW30 $\left(56-68^{\circ} \mathrm{C}\right.$ gradient annealing temperature). The PCR fragment was inserted into BamHI cut pKS vector. Mutagenesis war carried out as described in 4.2.1. E130Q was cloned into PacI cut pattB-5C plasmid by amplification with primers FW33 and FW34 $\left(58-70{ }^{\circ} \mathrm{C}\right.$ gradient annealing temperature $)$ to generate pattB-E130Q.

E. coli genomic DNA was used as template for generating the gene sequence of glyA, the bacterial SHMT gene. Here, primers FW27 and FW28 were used for amplification (55-67 ${ }^{\circ} \mathrm{C}$ gradient annealing temperature). This fragment was cloned into PacI cut pattB-5C to generate pattB-Bact.

For further cloning, a new vector had to be prepared, which also included the SHMT sequence from the first to the second start codon. For this a PCR was performed with pattB-SHMT-rescue as template, FW33 and FW51 as primers $\left(53-65^{\circ} \mathrm{C}\right.$ gradient annealing temperature). The PCR fragment was introduced into PacI cut pattB-5C, creating pattB-5C-new.

For generation of pattB-Bact2 E. coli cDNA was amplified with primers FW52 and FW53 under the same conditions as before and introduced into PacI cut pattB-5Cnew.

To create pattB- $\triangle \mathrm{CR} 44108$, the gene sequence of $S H M T$ was replaced by its coding sequence from the second start codon. Therefore the primer pair FW56/FW57 was used to amplify CDS from pGEX-SHMT-His using Pfu polymerase with the following program:

$\begin{array}{lcr}\text { Step } 1 \text { (Initial denaturation): } & 94^{\circ} \mathrm{C} & 3 \mathrm{~min} \\ \text { Step } 2 \text { (Denaturation): } & 94{ }^{\circ} \mathrm{C} & 30 \mathrm{sec} \\ \text { Step } 3 \text { (Annealing): } & 56-68^{\circ} \mathrm{C} & 30 \mathrm{sec} \\ \text { Step } 4 \text { (Elongation): } & 68^{\circ} \mathrm{C} & 90 \mathrm{sec} \\ \text { Step 5 (Repetition of cycles): } & \text { Steps } 2 \text { to } 4 & 35 \text { cycles } \\ \text { Step 6 (Final elongation): } & 68^{\circ} \mathrm{C} & 10 \mathrm{~min} \\ \text { Step 7 (Hold): } & 12^{\circ} \mathrm{C} & \infty\end{array}$

\section{Isolation and purification of DNA and RNA}

Plasmid mini and midi preparations were carried out using the kit from Macherey \& Nagel (Düren, Germany), purification of PCR products and digested DNA was done with the kit from Qiagen (Hilden, Germany), according to manufactur's instructions. 
Purification of DNA/RNA by phenol-chloroform extraction was carried out according to standard protocols [128].

\section{Quantitative Nanostring}

Experimental procedures were carried out by Dr. Hung-wei Sung. These methods are described in Sung et al., 2013 [151].

\section{RNA sequencing}

All experimental procedures preceding the actual RNA sequencing were carried out by Dr. Hung-wei Sung. RNA sequencing was performed by TAL with RNA isolated from staged embryo collections.

\subsubsection{Protein methods}

\section{Western blot}

Embryo collection:

Embryos were staged from 0 to 4 hours on apple-juice agar plates. Embryos were dechorionated in $50 \%$ Klorix bleach for 90 seconds, washed with water and collected into tubes, and spun down gently. The weight of the embryos was determined and their number calculated (1 mg 100 embryos). After adding 1x Lämmli buffer, the sample was heated to $95^{\circ} \mathrm{C}$ for $5 \mathrm{~min}$ and centrifuged at $14000 \mathrm{rpm}$ for $1 \mathrm{~min}$. The supernatant, the embryo lysate, was stored at $-20^{\circ} \mathrm{C}$ until use.

Western blot procedure was carried out as described in standard protocols [128]. Staining with Li-Cor secondary antibodies and detection using the Odyssey CLx Infrared Imaging System was carried out according to manufacturer's instructions.

\section{Expression and purification of proteins}

All recombinant proteins were expressed in E. coli BL21DE3.

\section{GST-SHMT-His:}

Protein expression was performed in $500 \mathrm{ml}$ of culture induced with $0.1 \mathrm{mM}$ IPTG at $20^{\circ} \mathrm{C}$ overnight. Cells were harvested by centrifuging at $4000 \mathrm{rpm}$ for $15 \mathrm{~min}$ at $4{ }^{\circ} \mathrm{C}$. Cells were re-suspended in lysis buffer and incubated with $1 \mathrm{mg} / \mathrm{ml}$ lysozyme and a pinch of DNase for 30 min on ice. Lysis was performed by first vortexing the suspension for $30 \mathrm{sec}$, followed by usage of a micro-fluidiser. To remove the insoluble fraction, 
lysate was centrifuged twice at $12000 \mathrm{rpm}$ for $20 \mathrm{~min}$ each. The cleared supernatant was filtered through a sterile micropore filter and passed through a Nickel-Sepharose prepacked column (HisTrap HP column) attached to an Äkta system, washed with appr. 10 column volumes (CV) of wash buffer and eluted with elution buffer. Afterwards the buffer with the eluted protein was exchanged to PBS using a PD10 column and the protein was concentrated. Glycerol was added to the protein sample to a final concentration of $10 \%$ and the protein was snap-frozen in liquid nitrogen and stored at $-80^{\circ} \mathrm{C}$. Purification of GST-SHMT-His is depicted in figure 59. The final amount of purified protein was about $10 \mathrm{mg}$ with a concentration of $2.6 \mathrm{mg} / \mathrm{ml}$.

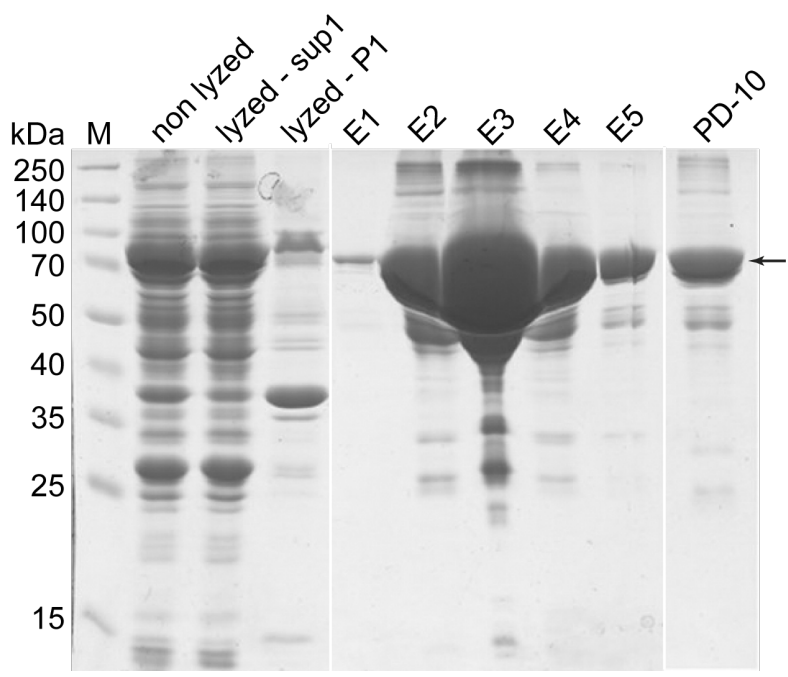

Figure 59: Purification of GST-SHMTHis with a HisTrap HP column. SDSPAGE showing samples from different steps of purification. The majority of the protein was soluble after lysation with lysozyme and micro-fluidiser. Elution fractions E2 to E5 were pooled and concentrated. A second concentration step after buffer exchange was performed to a concentration of $2.6 \mathrm{mg} / \mathrm{ml}$. Purified protein was aliquoted, frozen in liquid nitrogen and stored at $-80^{\circ} \mathrm{C}$. Protein band of GST-SHMT-His is marked by an arrow.

\section{GFP-HP1}

Protein expression was performed in $1 \mathrm{l}$ of culture with $0.1 \mathrm{mM}$ IPTG at $18^{\circ} \mathrm{C}$ overnight. Harvesting of cells, lysis and purification over a HisTrap HP column attached to an Äkta system was performed similarly to the purification of GST-SHMT-His, although with different buffer compositions (4.1.7). The yield of purified protein was $19.3 \mathrm{mg}$ at a concentration of $8.2 \mathrm{mg} / \mathrm{ml}$ (Figure 60 ).

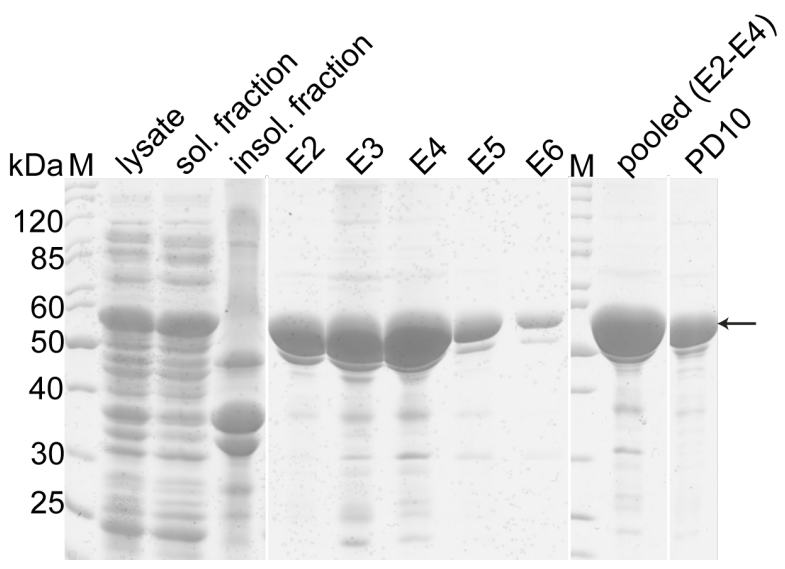

Figure 60: Purification of His-GFPHP1 with a HisTrap HP column. SDSPAGE showing samples from different steps of purification. The majority of the protein was soluble after lysation with lysozyme and micro-fluidiser. Elution fractions E2 to E4 were pooled and concentrated. A second concentration step after buffer exchange was performed to a concentration of $8.2 \mathrm{mg} / \mathrm{ml}$. The purified protein was aliquoted, frozen in liquid nitrogen and stored at $-80^{\circ} \mathrm{C}$. 


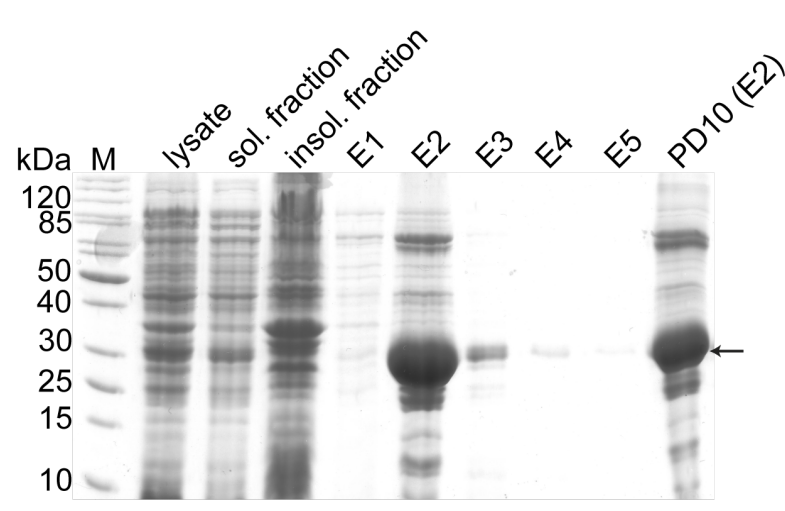

Figure 61: Purification of His-GFPlinker with a HisTrap HP column. SDSPAGE showing samples from different steps of purification. The majority of the protein was soluble after lysation with lysozyme and micro-fluidiser. Elution fraction E2 was run over a PD-10 column to exchange the buffer to storage buffer. The protein was concentrated to $6.8 \mathrm{mg} / \mathrm{ml}$. The purified protein was aliquoted, frozen in liquid nitrogen and stored at $-80^{\circ} \mathrm{C}$.

\section{GFP}

Expression and purification of His-GFP-linker was performed similarly to the purification of GFP-HP1-His, including the same buffer composition. The yield of purified protein was $11.2 \mathrm{mg}$ at a concentration of $6.8 \mathrm{mg} / \mathrm{ml}$ (Figure 61).

\subsubsection{Methods related to flies}

\section{Fly genetics}

X238 germline clones were generated by crossing X238 females to ovo19 or ovo18 males. To visualise nuclei in X238 germline clones, they were crossed to ovo males, which contained a transgene for GFP-Cad, RFP-Histone on the second chromosome. To express RpA70-eGFP in X238 germline clones, the RpA70-eGFP transgene (located on the third chromosome), was crossed into ovo19 background. To express GFP-PCNA in X238 germline clones, the UASp-GFP-PCNA transgene was crossed into X238Frt19A background, which then was crossed to ovo19 males containing a mat67 transgene on the second chromosome. X238, grapes double mutants were generated by crossing grapes (on the second chromosome) into the X238Frt18E background and crossing of the double mutation grapes, monk into the ovo18 background. Rescue constructs were introduced in X238 germline clones by crossing them into the ovo19 background. Overexpression of UASp-GFP-PCNA was introduced by crossing to mat67 and mat15 expressing males. ELMO germline clones were introduced by crossing to ovoD2L males. Kinesin-1 RNAi was performed by crossing to MTDGal4 flies.

\section{Generation of germline clones}

Induction of germline clones was performed mainly according to Chou and Perrimon, 1992 [37]. The heat shock, inducing the expression of flippase, was done at $37^{\circ} \mathrm{C}$ for $1 \mathrm{~h}$ on two consecutive days after hatching. 


\section{Generation of transgenic flies}

Transgenic flies were generated by the company GenetiVision (Houston, Texas, US).

\section{Fixation and immunostaining of Drosophila embryos}

Both methods were carried out according to Wenzl et al., 2010 [168].

\section{EdU labelling of embryos}

Embryos were staged to 0.5-2.5 hours. After dechorionation, the embryos were air dried for 3 to $5 \mathrm{~min}$. Afterwards, the net containing the embryos was placed in a 6 - or 12-well plate (depending on the size of the net) and heptane was added. The embryos were swirled to the middle of the net and bounced to form a monolayer of embryos. After incubation for $3 \mathrm{~min}$ the net was removed, heptane was wiped away, and the net was placed in Schneider's medium containing $50 \mathrm{mM}$ EdU. Incorporation of EdU was carried out at $25^{\circ} \mathrm{C}$ for $15 \mathrm{~min}$. Afterwards the net was removed, washed with heptane and transferred into a scintillation vial containing PBS and heptane. Fixation and storage were performed as already described.

\section{Fluorescence in situ hybridisation}

This method was performed according to Wenzl et al., 2010 [168]. Experimental procedures to prepare probes against the mRNAs of Slam and Kuk were carried out by Dr. Shuling Yan and Maria Kriebel, respectively.

\section{Microinjection}

Dechorionated embryos were lined up and dried in a desiccation chamber for $\sim 7 \mathrm{~min}$. After covering with halocarbon oil, the slide with embryos was placed under the microscope and injected posteriorly with protein or drug. The amount, which was injected, was depending on the pressure used. Imaging was either carried out immediately after injection $(\sim 1-3 \mathrm{~min})$ or after incubation of the embryos at room temperature (RT) for the desired time. Drugs and proteins were injected at the following concentrations: Colcemide $100 \mathrm{mg} / \mathrm{ml}$, Latrunculin A $100 \mathrm{mg} / \mathrm{ml}$, Jasplakinolide $1 \mathrm{mM}$, Y27632 $10 \mathrm{mM}$, sodium azide $0.02 \%$ (in water), $\alpha$-Amanitin $1 \mathrm{mg} / \mathrm{ml}$, H1-Alexa488 $2 \mathrm{mg} / \mathrm{ml}$, GFP-HP1 $8.2 \mathrm{mg} / \mathrm{ml}$ (starting concentration), GFP $6.8 \mathrm{mg} / \mathrm{ml}$. 


\section{Induction of clones in imaginal discs and follicle epithelium}

Female flies carrying an Frt18E site, either in combination with the X238 mutation or an otherwise WT chromosome, were crossed to males with the same Frt site and an nls-GFP construct. To induce clones in imaginal discs, heat shocks (at $37^{\circ} \mathrm{C}$ for 1 hour each) were carried out on two consecutive days in L1 and L2 larvae (24-48 and 48-72 hours after egg laying). Imaginal discs were prepared from late L3 larvae. Induction of clones in the follicle epithelium was performed by heat shock on two consecutive days in adult flies. Ovaries were dissected 3 to 5 days after induction.

\section{Preparation and staining of imaginal discs and ovaries}

Imaginal discs:

L3 larvae were placed to a drop of PBS on a cover slide. Both ends of the larvae were hold with tweezers and the larvae was pulled apart. After careful removement of the digestive system and fat tissue, the opening of the anterior part of the larvae was widened by gently pulling at larvae skin. The anterior part was inverted to make the discs accessible. The colorless discs were collected in tubes fused to a small net on ice. After preparation of 7 to 10 larvae per staining, the imaginal discs were transferred into a standard tube. They were fixed in $4 \%$ paraformaldehyde in PBS for 40 min at RT with constant rotating. They were washed with PBS containing $0.3 \%$ Triton X-100 for $20 \mathrm{~min}$, and blocked with $5 \%$ BSA in PBS+0.3\% Triton X-100 for 1 hour at RT. Either protein staining was performed at described or it was continued with staining with GFP booster (1:500) for 2 hours at RT, protected from light. If protein staining was carried out, the staining with GFP booster was done together with secondary antibody incubation. After washing four times $15 \mathrm{~min}$, DAPI staining was carried out for $7 \mathrm{~min}$. Another washing period was followed by mounting in aquapolymount mounting medium. During mounting, the discs were arranged and all unnecessary parts from the larvae were removed.

Ovaries:

Females flies were anaesthetised and their heads were removed. Tweezers were attached to the middle of the fly and the tip of the abdomen, and the fly was pulled apart. Ovaries were pushed out of the fly body and collected in tubes on ice. After dissecting $\sim 10$ flies per staining, the ovaries were transferred into a scintillation vial with $9.5 \mathrm{ml}$ of PBS and $0.5 \mathrm{ml}$ of $37 \%$ formaldehyde and fixed for 20 min with constant shaking. Afterwards the ovaries were transferred into a tube, rinsed three times and washed three times 15 min with PBT with constant rotating. Staining with GFP booster (1:500) was carried out for $30 \mathrm{~min}$ at RT, protected from light. After another washing period, the ovaries were stained with DAPI for 1 min. A final washing period was following by mounting in aquapolymount mounting medium, thereby arranging the ovaries on the slide and removing unnecessary parts of the flies. 


\subsubsection{Microscopy and related methods}

\section{Live imaging}

Embryo were dechorionated, aligned on a piece of apple juice agar plate, clued on a coverslip, desiccated if necessary, and covered with halocarbon oil. Fluorescent liveimaging of RpA70eGFP was performed at the LSM microscope with a $63 \mathrm{x}$ oil objective. All other fluorescent live-imaging experiments were carried out at the spinning disc microscope with $25 \mathrm{x}, 40 \mathrm{x}$, or $100 \mathrm{x}$ oil objectives. The temperature was kept between $20^{\circ} \mathrm{C}$ and $23^{\circ} \mathrm{C}$.

Time-lapse recordings with DIC optics were performed with a light intensity of $2.7 \mathrm{~V}$, an exposure time of $100 \mathrm{~ms}$ and a frame interval of $30 \mathrm{sec}$ to $1 \mathrm{~min}$. For fluorescence recording of Histone-RFP or GFP-PCNA expressing or H1-Alexa488 or GFP-HP1 injected embryos, movies were taken with $50 \%$ laser intensity, $100 \mathrm{~ms}$ exposure time, one $\mathrm{Z}$ plane and a frame interval of $1 \mathrm{~min}$. To record Sas6- or Sas4-GFP expression in the centrosomes, time-lapse imaging was performed with the following settings: $75 \%$ laser intensity, $30 \mathrm{~ms}$ exposure time, seven to nine $\mathrm{Z}$ sections, frame rate of $0.02-1 \mathrm{sec}$ for up to $20 \mathrm{~min}$. Time-lapse recordings with a frame rate of $1 \mathrm{sec}$ were performed with a 40x oil objective, fast recordings with a frame rate of $20 \mathrm{~Hz}$ were carried out with a 100x oil objective and the use of an emCCD camera. Fast recordings were started shortly after start of centrosome duplication, when they stopped moving apical-basally. For low illumination conditions, the laser intensity was reduced to $25 \%\left(=\frac{1}{3}\right.$ of the normal intensity).

\section{Imaging of fixed samples}

Fixed and immunostained embryos were imaged at the LSM microscope with 25x and $63 \mathrm{x}$ water objectives. Laser intensities were set to optimal illumination conditions, the resolution was set to half of the objectives maximal resolution value to obtain optimal signal covering. The pixel dwell time, the time, in which the laser illuminates one pixel, was set to values between 1-2 $\mu$ sec. Each channel was recorded separately.

\section{Fluorescence recovery after photo-bleaching}

Bleaching of Utrophin-GFP in actin caps was carried out at the LSM microscope. In a given area the laser intensity was set to $100 \%$, and 50 iterations were performed at a scan speed of 5 . Time-lapse recordings with reduced laser intensity were performed for 15 min. 


\section{Image processing and data analysis}

The $C Z I$ files, which were obtained from spinning disc microscopy were exported into omeTIFF files, and imported into ImageJ as 16-bit TIFF stacks. LSM files could directly be processed in ImageJ.

ImageJ was used to reduce background fluorescence and adjust image colour by using the Brightness/Contrast and Channels tool options. Single images were arranged to figures using Adobe Photoshop and labelling of figures was carried out with Adobe Illustrator.

Syncytial cell cycle number was determined either from DIC movies or from movies recorded with a fluorescent nuclear marker. Determination from DIC movies was done by counting the number of interphases, in which the nuclei become visible in the embryos. Determination from fluorescent movies was carried out by labelling a squared section of defined size in ImageJ and counting the nuclei at least half located in this area.

FRAP experiments were analysed with ImageJ. Using the measurement tool, the fluorescence intensity of a small membrane area outside and inside of the bleached region was measured. 



\section{Appendix}

Counts obtained in Nanostring experiments

Table 25: Normalised counts of internal Nanostring CodeSet controls.

\begin{tabular}{|c|c|c|c|c|c|c|c|c|}
\hline \multirow[b]{2}{*}{ Gene } & \multicolumn{4}{|c|}{ WT } & \multicolumn{4}{|c|}{$\mathrm{X} 238$} \\
\hline & pre & c. 12 & c. 13 & c. 14 & pre & c 12 & c 13-e & c. $13-1$ \\
\hline NEG_A & 1 & 7 & 3.5 & 2 & 4 & 2 & 3.5 & 4.5 \\
\hline $\mathrm{NEG}{ }_{-} \mathrm{B}$ & 5 & 1 & 1 & 1 & 3 & 1 & 3 & 2.5 \\
\hline $\mathrm{NEG}_{-}^{-} \mathrm{C}$ & 1 & 2 & 1 & 2.5 & 2 & 1 & 1.5 & 3 \\
\hline $\mathrm{NEG}_{-} \mathrm{D}$ & 1 & 2 & 1.5 & 1 & 2 & 3 & 2.5 & 1 \\
\hline $\mathrm{NEG}_{-} \mathrm{E}$ & 5 & 8 & 4.5 & 3 & 2 & 5 & 4.5 & 3.5 \\
\hline $\mathrm{NEG}_{-} \mathrm{F}$ & 2 & 3 & 2 & 3 & 4 & 6 & 4 & 6 \\
\hline NEG_G & 1 & 1 & 1.5 & 1 & 9 & 4 & 2.5 & 3 \\
\hline $\mathrm{NEG}_{-}^{-} \mathrm{H}^{\prime}$ & 2 & 4 & 4.5 & 6.5 & $\begin{array}{ll}1 & 3\end{array}$ & 5 & 3 & 6.5 \\
\hline POS_A & 7498 & 13790 & 11918.5 & 15226 & : 11516 & 11839 & 14314 & 14059.5 \\
\hline $\mathrm{POS}_{-} \mathrm{B}$ & 2555 & 4663 & 3926.5 & 5132 & 3896 & 4034 & 4943 & 4666.5 \\
\hline $\mathrm{POS}_{-} \mathrm{C}$ & 750 & 1398 & 1207 & 1455 & 1182 & 1168 & 1366 & 1395 \\
\hline $\mathrm{POS}_{-} \mathrm{D}$ & 130 & 271 & 242 & 280 & $\begin{array}{l}1 \\
1\end{array}$ & 207 & 245 & 264.5 \\
\hline POS_E & 24 & 92 & 57.5 & 79.5 & 70 & 59 & 69 & 71 \\
\hline POS_F & 19 & 33 & 18 & 27 & 23 & 27 & 32 & 25 \\
\hline
\end{tabular}

Table 26: Normalised counts for the housekeeping genes Rpl21, RpLP2, and RpL32.

\begin{tabular}{l|cccccccc}
\hline & \multicolumn{4}{c}{ WT } & \multicolumn{4}{c}{ X238 } \\
\cline { 2 - 5 } \cline { 6 - 9 } Gene & pre & c. 12 & c. 13 & c. 14 & pre & c 12 & c 13-e & c. 13-1 \\
\hline RPL32 & 11136.7 & 11322.14 & 11129.28 & 12006.38 & 11009.2 & 11200.98 & 11827.99 & 11301.33 \\
RpLP2 & 14939.17 & 15141.67 & 14795.75 & 13348.27 & 16584.28 & 15373.16 & 14223.99 & 15533.01 \\
Rpl21 & 24589.95 & 23863.73 & 24861.73 & 25559.74 & 22407.22 & 23758.63 & 24344.48 & 23307.6 \\
\hline
\end{tabular}


Table 27: Normalised counts for zygotic genes.

\begin{tabular}{|c|c|c|c|c|c|c|c|c|}
\hline \multirow[b]{2}{*}{ Gene } & \multicolumn{4}{|c|}{ WT } & \multicolumn{4}{|c|}{$\mathrm{X} 238$} \\
\hline & pre & c. 12 & c. 13 & c. 14 & pre & c 12 & c 13-e & c. $13-1$ \\
\hline dpp & 3.08 & 12.89 & 63.09 & 87.18 & 1.17 & 22.32 & 56.44 & 69.02 \\
\hline eve & 1.54 & 71.99 & 165.32 & 161.04 & 7.05 & 74 & 130.17 & 195.52 \\
\hline frs & 3.08 & 181.58 & 1423.54 & 8889.58 & 14.1 & 444 & 795.43 & 3003.51 \\
\hline halo & 27.69 & 495.3 & 2339.29 & 8791.25 & 42.29 & 903.27 & 1820.77 & 4214.03 \\
\hline hb a & 4.61 & 249.26 & 829.79 & 479.44 & 11.75 & 266.63 & 488.72 & 696.79 \\
\hline kni & 1.54 & 21.49 & 70.65 & 84.12 & 4.7 & 38.76 & 36.36 & 130.36 \\
\hline $\operatorname{sis} \mathrm{A}$ & 21.54 & 298.96 & 402.33 & 325.33 & 14.1 & 244.32 & 329.14 & 1206.34 \\
\hline slam & 52.3 & 295.46 & 1006.56 & 297.17 & 345.36 & 370 & 634.63 & 1460.13 \\
\hline Krüppel & 4.61 & 39.75 & 257.76 & 632.9 & 17.62 & 75.17 & 164.28 & 497.7 \\
\hline nullo & 1.54 & 10.74 & 13.69 & 14.89 & 3.52 & 11.75 & 14.91 & 7.03 \\
\hline sxl & 12.31 & 16.12 & 12.86 & 36.01 & 43.46 & 12.92 & 20.54 & 47.08 \\
\hline $\operatorname{sisB} / \mathrm{SC}$ & 4.61 & 482.41 & 932.09 & 649.44 & 7.05 & 651.9 & 1077.27 & 517.67 \\
\hline zen & 13.84 & 318.03 & 797.26 & 1501.79 & 31.72 & 471.01 & 624.46 & 983.46 \\
\hline zld & 163.05 & 326.62 & 671.53 & 143.49 & 1121.83 & 370 & 785.81 & 582.66 \\
\hline
\end{tabular}

Table 28: Normalised counts for transcripts of zygotically degraded mRNAs.

\begin{tabular}{|c|c|c|c|c|c|c|c|c|}
\hline \multirow[b]{2}{*}{ Gene } & \multicolumn{4}{|c|}{ WT } & \multicolumn{4}{|c|}{ X238 } \\
\hline & pre & c. 12 & c. 13 & c. 14 & pre & c 12 & c 13-e & c. $13-1$ \\
\hline smg & 3607.12 & 10106.98 & 10722.2 & 2286.67 & 11756.3 & 7180.33 & 9857.79 & 4955.47 \\
\hline string & 515.3 & 2043.53 & 1589.16 & $222.45 !$ & 2766.4 & 1751.33 & 1984.17 & 982.09 \\
\hline twine & 1116.75 & 1502.03 & 1446.42 & 1037.94 & 1267.49 & 1186.35 & 1426.55 & 782.47 \\
\hline CG5966 & 176.9 & 349.18 & 270.81 & 56.4 & 464 & 313.62 & 272.81 & 150.77 \\
\hline $\mathrm{CycB}$ & 1618.21 & 4953.03 & 4838.26 & 833.09 & 5515.17 & 4451.73 & 4512.39 & 2893.09 \\
\hline bicoid & 463 & 890.69 & 976.11 & $247.02 !$ & 1408.45 & 756.44 & 912.89 & 532.98 \\
\hline
\end{tabular}


Table 29: Normalised counts of maternally degraded mRNAs.

\begin{tabular}{|c|c|c|c|c|c|c|c|c|}
\hline \multirow[b]{2}{*}{ Gene } & \multicolumn{4}{|c|}{ WT } & \multicolumn{4}{|c|}{ X238 } \\
\hline & pre & c. 12 & c. 13 & c. 14 & pre & c 12 & c 13-e & c. $13-1$ \\
\hline Сyp6a19 & $\begin{array}{l}3097.97 \\
\end{array}$ & 400.76 & 49.03 & 10.24 & 3573.41 & 473.36 & 280.53 & 56.5 \\
\hline GstE6 & $\quad 226.12$ & 46.2 & 13.8 & 6.05 & 552.1 & 75.17 & 41.7 & 39.53 \\
\hline Nanos & 3164.11 & 3016.94 & 1308.07 & 114.91 & 6911.88 & 2963.51 & 2332.03 & 758.02 \\
\hline Tpi & $\begin{array}{l}2179.65 \\
\end{array}$ & 1067.96 & 205.44 & 32.62 & 7259.59 & 1403.65 & 599.11 & 207.85 \\
\hline cort & 207.66 & 30.08 & 13.97 & 3.53 & 345.36 & 22.32 & 24.72 & 13.11 \\
\hline exu & 4316.24 & 713.41 & 186.16 & 37.28 & 6108.39 & 664.82 & 478.71 & 146.39 \\
\hline
\end{tabular}


Lists of candidates identified in RNA sequencing

Table 30: Genes differentially expressed in presyncytial X238 mutants compared to WT embryos.

\begin{tabular}{|c|c|c|c|c|c|}
\hline Gene name & $\mathrm{FC}_{\log 2}$ & FDR & Gene name & $\mathrm{FC}_{\log 2}$ & FDR \\
\hline CG3038 & -4.30 & 5.43E-193 & GstZ1 & 1.08 & $3.94 \mathrm{E}-14$ \\
\hline pn & -2.79 & $1.42 \mathrm{E}-108$ & Idh & 1.12 & $3.24 \mathrm{E}-77$ \\
\hline CG30440 & -1.79 & $1.29 \mathrm{E}-43$ & CR43460 & 1.14 & $5.46 \mathrm{E}-13$ \\
\hline Tollo & -1.58 & $2.61 \mathrm{E}-30$ & Grd & 1.15 & $1.69 \mathrm{E}-12$ \\
\hline fuss & -1.56 & $1.75 \mathrm{E}-24$ & CG12991 & 1.18 & $1.14 \mathrm{E}-11$ \\
\hline CR43836 & -1.52 & $4.25 \mathrm{E}-29$ & GLaz & 1.20 & $7.33 \mathrm{E}-16$ \\
\hline l(1)G0232 & -1.50 & $1.74 \mathrm{E}-112$ & CG32017 & 1.21 & $3.81 \mathrm{E}-13$ \\
\hline Pde9 & -1.47 & $1.61 \mathrm{E}-17$ & CG4095 & 1.22 & $1.46 \mathrm{E}-13$ \\
\hline CG34353 & -1.43 & $1.49 \mathrm{E}-17$ & GstD9 & 1.22 & $1.19 \mathrm{E}-28$ \\
\hline CG10962 & -1.41 & $5.82 \mathrm{E}-24$ & CG12112 & 1.24 & $4.50 \mathrm{E}-41$ \\
\hline CG4733 & -1.33 & $3.03 \mathrm{E}-16$ & CG13032 & 1.24 & $4.27 \mathrm{E}-14$ \\
\hline CG5096 & -1.29 & $7.59 \mathrm{E}-14$ & CG42450 & 1.25 & $9.49 \mathrm{E}-14$ \\
\hline CG17352 & -1.27 & $1.76 \mathrm{E}-14$ & wake & 1.27 & $3.70 \mathrm{E}-13$ \\
\hline otk-2 & -1.23 & $4.26 \mathrm{E}-13$ & CG18473 & 1.33 & $1.59 \mathrm{E}-14$ \\
\hline FASN2 & -1.18 & $1.14 \mathrm{E}-11$ & CG2641 & 1.37 & $2.52 \mathrm{E}-15$ \\
\hline CG5455 & -1.18 & $1.64 \mathrm{E}-18$ & MtnA & 1.38 & $1.66 \mathrm{E}-15$ \\
\hline CR12460 & -1.17 & 3.37E-11 & CG10208 & 1.42 & $2.42 \mathrm{E}-16$ \\
\hline CG12581 & -1.17 & $3.50 \mathrm{E}-11$ & meso18E & 1.54 & $1.84 \mathrm{E}-20$ \\
\hline CG17928 & -1.16 & $4.00 \mathrm{E}-12$ & CG10407 & 1.62 & $1.48 \mathrm{E}-21$ \\
\hline Sclp & -1.14 & $1.21 \mathrm{E}-10$ & swaPsi & 1.67 & 7.63E-29 \\
\hline Pino & -1.13 & $1.28 \mathrm{E}-51$ & CR42861 & 1.78 & $1.95 \mathrm{E}-35$ \\
\hline mbl & -1.13 & $2.52 \mathrm{E}-11$ & Lsd-1 & 1.87 & $1.53 \mathrm{E}-32$ \\
\hline pyd3 & -1.12 & $1.63 \mathrm{E}-10$ & hiw & 1.90 & $4.18 \mathrm{E}-39$ \\
\hline PGRP-LC & -1.07 & $2.04 \mathrm{E}-09$ & Sodh-2 & 1.99 & $1.53 \mathrm{E}-32$ \\
\hline CG9684 & -1.06 & $1.17 \mathrm{E}-50$ & CG43163 & 2.19 & $4.18 \mathrm{E}-39$ \\
\hline CG1234 & -1.03 & $6.82 \mathrm{E}-44$ & Lin29 & 2.24 & $2.59 \mathrm{E}-44$ \\
\hline CG42565 & -1.02 & $9.18 \mathrm{E}-09$ & CG14879 & 2.35 & $5.30 \mathrm{E}-45$ \\
\hline egr & -1.00 & $2.36 \mathrm{E}-08$ & CG13532 & 2.38 & $4.03 \mathrm{E}-46$ \\
\hline CG4896 & 1.01 & $8.58 \mathrm{E}-15$ & pst & 2.45 & $3.15 \mathrm{E}-64$ \\
\hline CG5390 & 1.02 & $8.56 \mathrm{E}-09$ & Сур4p2 & 2.55 & $4.10 \mathrm{E}-53$ \\
\hline GstE6 & 1.02 & $3.89 \mathrm{E}-12$ & Dscam4 & 2.69 & $2.28 \mathrm{E}-59$ \\
\hline Reck & 1.02 & $5.62 \mathrm{E}-09$ & CG11700 & 2.73 & $1.42 \mathrm{E}-101$ \\
\hline Mur2B & 1.03 & $4.95 \mathrm{E}-10$ & Zip89B & 2.95 & $5.06 \mathrm{E}-73$ \\
\hline CG4398 & 1.04 & $7.30 \mathrm{E}-11$ & GstD3 & 4.28 & $5.76 \mathrm{E}-181$ \\
\hline RhoGEF64C & 1.05 & $1.01 \mathrm{E}-09$ & & & \\
\hline
\end{tabular}


Table 31: Genes differentially expressed in X238 mutants of cycle 13 compared to cellularising WT embryos. Reduced list of the 38 most up- and down-regulated candidates.

\begin{tabular}{|c|c|c|c|c|c|}
\hline Gene name & $\mathrm{FC}_{\log 2}$ & FDR & Gene name & $\mathrm{FC}_{\log 2}$ & FDR \\
\hline CG3038 & -6.72 & $1.53 \mathrm{E}-121$ & CG10208 & 2.71 & $1.05 \mathrm{E}-17$ \\
\hline CG54263 & -6.67 & $2.21 \mathrm{E}-77$ & CG10407 & 2.73 & $9.38 \mathrm{E}-21$ \\
\hline CG34353 & -4.95 & $7.86 \mathrm{E}-24$ & Rpi & 2.73 & $3.87 \mathrm{E}-26$ \\
\hline CG15236 & -4.75 & $7.08 \mathrm{E}-25$ & stg & 2.73 & $1.96 \mathrm{E}-30$ \\
\hline pn & -4.60 & $1.55 \mathrm{E}-69$ & Grd & 2.75 & 7.92E-06 \\
\hline CG13928 & -4.12 & $5.11 \mathrm{E}-23$ & rux & 2.79 & $9.85 \mathrm{E}-15$ \\
\hline CG17928 & -4.08 & $9.99 \mathrm{E}-14$ & swaPsi & 2.81 & $5.77 \mathrm{E}-12$ \\
\hline сро & -3.71 & $7.70 \mathrm{E}-11$ & Ice2 & 2.82 & $8.34 \mathrm{E}-47$ \\
\hline Or9a & -3.46 & 7.09E-10 & pes & 2.86 & $6.58 \mathrm{E}-22$ \\
\hline CG42816 & -3.44 & $3.42 \mathrm{E}-12$ & CG3040 & 2.90 & $1.92 \mathrm{E}-13$ \\
\hline CG34383 & -3.42 & $5.32 \mathrm{E}-18$ & CG6912 & 2.91 & 2.08E-07 \\
\hline tutl & -3.12 & $8.33 \mathrm{E}-15$ & Idh & 2.97 & $1.16 \mathrm{E}-32$ \\
\hline Cyp6d2 & -3.12 & $5.73 \mathrm{E}-15$ & wus & 2.97 & $5.67 \mathrm{E}-34$ \\
\hline $\operatorname{Trs} 23$ & -3.10 & $6.95 \mathrm{E}-31$ & Mitf & 3.00 & $9.26 \mathrm{E}-20$ \\
\hline ASPP & -3.10 & $4.20 \mathrm{E}-16$ & Pp2B-14D & 3.02 & $1.00 \mathrm{E}-40$ \\
\hline CG5080 & -3.09 & $1.47 \mathrm{E}-15$ & Cyp6d4 & 3.04 & $1.79 \mathrm{E}-17$ \\
\hline CG16700 & -3.05 & $4.38 \mathrm{E}-10$ & Usf & 3.08 & $5.37 \mathrm{E}-38$ \\
\hline CG12896 & -3.04 & $1.77 \mathrm{E}-11$ & CG14218 & 3.11 & $1.13 \mathrm{E}-07$ \\
\hline nkd & -3.01 & 5.39E-10 & CG12024 & 3.11 & $1.14 \mathrm{E}-15$ \\
\hline CG5758 & -2.95 & $3.68 \mathrm{E}-10$ & CG9925 & 3.12 & $1.71 \mathrm{E}-124$ \\
\hline pyd3 & -2.89 & $5.09 \mathrm{E}-23$ & CR42861 & 3.20 & $7.06 \mathrm{E}-39$ \\
\hline Ugt58Fa & -2.88 & 2.03E-07 & CR33319 & 3.31 & $2.86 \mathrm{E}-20$ \\
\hline CG7465 & -2.86 & $3.62 \mathrm{E}-08$ & CR43614 & 3.33 & $6.75 \mathrm{E}-13$ \\
\hline Pde9 & -2.86 & $2.09 \mathrm{E}-10$ & pst & 3.47 & $1.59 \mathrm{E}-32$ \\
\hline ey & -2.73 & $1.13 \mathrm{E}-11$ & gammaTub37C & 3.67 & $1.60 \mathrm{E}-34$ \\
\hline stj & -2.70 & 8.03E-08 & CG32017 & 3.68 & $3.57 \mathrm{E}-20$ \\
\hline Cyp18a1 & -2.69 & $5.54 \mathrm{E}-07$ & Tgt & 3.69 & $9.01 \mathrm{E}-30$ \\
\hline zye & -2.68 & $8.29 \mathrm{E}-08$ & Sodh-2 & 4.00 & $5.60 \mathrm{E}-57$ \\
\hline Atx-1 & -2.64 & $5.93 \mathrm{E}-11$ & CG3626 & 4.12 & $3.10 \mathrm{E}-53$ \\
\hline Jhe & -2.62 & $2.68 \mathrm{E}-18$ & Pvf1 & 4.15 & $2.14 \mathrm{E}-100$ \\
\hline $\mathrm{v}(2) \mathrm{k} 05816$ & -2.57 & $1.26 \mathrm{E}-09$ & Zip89B & 4.75 & $6.74 \mathrm{E}-20$ \\
\hline CR12460 & -2.51 & $3.32 \mathrm{E}-27$ & CG2641 & 4.81 & $2.50 \mathrm{E}-37$ \\
\hline stl & -2.51 & $1.94 \mathrm{E}-08$ & CG14879 & 5.28 & $3.10 \mathrm{E}-30$ \\
\hline eve & -2.49 & $1.83 \mathrm{E}-20$ & Dscam4 & 5.34 & $1.10 \mathrm{E}-39$ \\
\hline Cyt-b5-r & -2.47 & $6.30 \mathrm{E}-05$ & Cyp4p2 & 5.59 & $3.04 \mathrm{E}-80$ \\
\hline lea & -2.47 & $4.54 \mathrm{E}-16$ & CG13532 & 5.72 & $3.26 \mathrm{E}-37$ \\
\hline Ca-beta & -2.46 & $6.47 \mathrm{E}-05$ & CG15047 & 5.95 & $9.14 \mathrm{E}-72$ \\
\hline
\end{tabular}





\section{Bibliography}

[1] R. T. Abraham. 'Cell cycle checkpoint signaling through the ATM and ATR kinases.' Genes 8 Development 15 (2001), pp. 2177-2196.

[2] S. Acharya, P. Laupsien, C. Wenzl, S. Yan and J. Großhans. 'Function and dynamics of slam in furrow formation in early Drosophila embryo'. Developmental Biology 386.2 (2014), pp. 371-384.

[3] K. Afshar, B. Stuart and S. A. Wassermann. 'Functional analysis of the Drosophila diaphanous FH protein in early embryonic development.' Dev. Camb. Engl. 127 (2000), pp. 1887-1897.

[4] S. Agrawal, A. Kumar, V. Srivastava and B. N. Mishra. 'Cloning, expression, activity and folding studies of serine hydroxymethyltransferase: A target enzyme for cancer chemotherapy.' Journal of Molecular Microbiology and Biotechnology 6.2 (2003), pp. 67-75.

[5] R. Albertson, B. Riggs and W. Sullivan. 'Membrane traffic: A driving force in cytokinesis.' Trends in Cell Biology 15.2 (2005), pp. 92-101.

[6] D. D. Anderson, J. Y. Eoms and P. J. Stovers. 'Competition between sumoylation and ubiquitination of serine hydroxymethyltransferase 1 determines its nuclear localization and its accumulation in the nucleus.' Journal of Biological Chemistry 287.7 (2012), pp. 4790-4799.

[7] D. D. Anderson, C. F. Woeller and P. J. Stover. 'Small ubiquitin-like modifier1 (SUMO-1) modification of thymidylate synthase and dihydrofolate reductase.' Clin Chem Lab Med 45 (2007), pp. 1760-1763.

[8] D. D. Anderson, C. M. Quintero and P. J. Stover. 'Identification of a de novo thymidylate biosynthesis pathway in mammalian mitochondria.' Proceedings of the National Academy of Sciences of the United States of America 108.37 (2011), pp. 15163-15168.

[9] D. D. Anderson and P. J. Stover. 'SHMT1 and SHMT2 are functionally redundant in nuclear de novo thymidylate biosynthesis.' PLoS ONE 4.6 (2009), e5839.

[10] D. D. Anderson, C. F. Woeller, E.-P. Chiang, B. Shane and P. J. Stover. 'Serine Hydroxymethyltransferase Anchors de Novo Thymidylate Synthesis Pathway to Nuclear Lamina for DNA Synthesis.' Journal of Biological Chemistry 287.10 (2012), pp. 7051-7062. 
[11] N. Appaji Rao, R. Talwar and H. S. Savithri. 'Molecular organization , catalytic mechanism and function of serine hydroxymethyltransferase - a potential target for cancer chemotherapy.' International Journal of Biochemistry and Cell Biology 32 (2000), pp. 405-416.

[12] D. R. Appling. 'Compartmentation of folate-mediated one-carbon metabolism in eukaryotes.' FASEB Journal 5 (1991), pp. 2645-2651.

[13] J. Baker, W. E. Theurkauf and G. Schubiger. 'Dynamic changes in microtubule configuration correlate with nuclear migration in the preblastoderm Drosophila embryo.' Journal of Cell Biology 122 (1993), pp. 113-121.

[14] J. Bartek and J. Lukas. 'DNA damage checkpoints: from initiation to recovery or adaptation.' Current Opinion in Cell Biology 19 (2007), pp. 238-245.

[15] A. Bashirulllah, S. R. Halsell, R. L. Cooperstock, M. Kloc, A. Karaiskakis, W. W. Fisher, W. Fu, J. K. Hamilton, L. D. Etkin and H. D. Lipshitz. 'Joint action of two RNA degradation pathways controls the timing of maternal transcript elimination at the midblastula transition in Drosophila melanogaster.' EMBO Journal 18 (1999), pp. 2610-2620.

[16] A. E. Beaudin, E. V. Abarinov, D. M. Noden, C. A. Perry, S. Chu, S. P. Stabler, R. H. Allen and P. J. Stover. 'Shmt1 and de novo thymidylate biosynthesis underlie folate-responsive neural tube defects in mice.' American Journal of Clinical Nutrition 93 (2011), pp. 789-798.

[17] A. E. Beaudin and P. J. Stover. 'Insights into metabolic mechanisms underlying folate-responsive neural tube defects: A minireview'. Birth Defects Research (Part A ) 85.4 (2009), pp. 274-284.

[18] H. Beck, V. Nähse, M. S. Y. Larsen, P. Groth, T. Clancy, M. Lees, M. Jørgensen, T. Helleday, R. G. Syljuåsen and C. S. Sørensen. 'Regulators of cyclin-dependent kinases are crucial for maintaining genome integrity in S phase.' Journal of Cell Biology 188.5 (2010), pp. 629-638.

[19] K. Beckmann, C. Dzuibany, K. Bichner, H. Fock, R. Hell, A. Migge and T. W. Becker. 'Photosynthesis and fluorescence quenching and the messenger RNA of plastidic glutamine synthetase or of mitochondrial serine hydroxymethyltransferase (SHMT) in the leaves of the wild type end of the SHMT deficient Stm mutant of Arabidopsis thalliana in relation to the rate of photorespiration.' Planta 202 (1997), pp. 379-386.

[20] S. E. Bergsten and E. R. Gravis. 'Role for mRNA localization in translational activation but not spatial restriction of nanos RNA.' Development 122 (1999), pp. $2791-28000$.

[21] H. M. Berman, J. Westbrook, Z. Feng, G. Gilliland, T. N. Bhat, H. Weissig, I. N. Shindyalov and P. E. Bourne. 'The Protein Data Bank.' Nucleic acids research 28.1 (2000), pp. 235-242. 
[22] V. Besson, M. Neuburger, F. Rebeille and R. Douce. 'Evidence for three serine hydroxymethyltransferases in green leaf cells. Purification and characterization of the mitochondrial and chloroplastic isoforms.' Plant Physiol Biochem 33 (1995), pp. 665-673.

[23] A. N. Bhatt, M. Y. Khan and V. Bhakuni. 'The C-terminal domain of dimeric serine hydroxymethyltransferase plays a key role in stabilization of the quaternary structure and cooperative unfolding of protein: domain swapping studies with enzymes having high sequence identity.' Protein science 13.8 (2004), pp. 21842195 .

[24] X. Bi, S. C. Wei and Y. S. Rong. 'Telomere protection without a telomer- ase; the role of ATM and Mre11 in Drosophila telomere maintenance.' Current Biology 14 (2004), pp. 1348-1353.

[25] B. C. Blount, M. M. Mack, C. M. Wehr, J. T. MacGregor, R. A. Hiatt, G. Wang, S. N. Wickramasinghe, R. B. Everson and B. N. Ames. 'Folate Deficiency Causes Uracil Misincorporations into Human DNA and Chromosome Breakage: Implications for Cancer and Neuronal Damage.' Proceedings of the National Academy of Sciences of the United States of America 94.7 (1997), pp. 3290-3295.

[26] M. D. Blower and G. H. Karpen. 'The role of Drosophila CID in kinetochore formation, cell-cycle progression and heterochromatin interactions.' Nature cell biology 3.8 (2001), pp. 730-739.

[27] A. B. Blumenthal, H. J. Kriegstein and D. S. Hogness. 'The units of DNA replication in Drosophila melanogaster chromosomes.' Cold Spring Harb. Symp. Quant. Biol. 38 (1974), pp. 205-223.

[28] S. A. Blythe and E. F. Wieschaus. 'Zygotic Genome Activation Triggers the DNA Replication Checkpoint at the Midblastula Transition.' Cell 160.6 (2015), pp. 1169-1181.

[29] M. N. Boddy, B. Furnari, O. Mondesert and P. Russell. 'Replication checkpoint enforced by kinases Cds1 and Chk1.' Science 280 (1998), pp. 909-912.

[30] M. Boddy and P. Russell. 'DNA replication checkpoint control.' Front. Biosci. 4 (1999), pp. 841-848.

[31] M. H. Brodsky, B. T. Weinert, G. Tsang, Y. S. Rong, N. M. Mcginnis, K. G. Golic, D. C. Rio and G. M. Rubin. 'Drosophila melanogaster MNK/Chk2 and p53 Regulate Multiple DNA Repair and Apoptotic Pathway following DNA Damage.' Molecular and cellular biology 24.3 (2004), pp. 1219-1231.

[32] E. J. Brown and D. Baltimore. 'ATR disruption leads to chromosomal frag- mentation and early embryonic lethality.' Genes \& Development 14 (2000), pp. 397402. 
[33] Y. Burdirahardja and P. Gönczy. 'Coupling the cell cycle to development.' Development 136 (2009), pp. 2861-2872.

[34] K. S. Chen, P. H. Gunaratne, J. D. Hoheisel, I. G. Young, G. L. Miklos, F. Greenberg, L. G. Shaffer, H. D. Campbell and J. R. Lupski. 'The human homologue of the Drosophila melanogaster flightless-I gene (fifI) maps within the SmithMagenis microdeletion critical region in 17pll.2.' Am J Hum Genet 56 (1995), pp. $175-182$.

[35] M. A. Chesarone, A. G. DuPage and B. L. Goode. 'Unleashing formins to remodel the actin and microtubule cytoskeletons.' Nat. Rev. Mol. Cell Biol. 11 (2010), pp. $62-74$.

[36] C. Chevillard, D. Le Paslier, E. Passage, P. Ougen, A. Billault, S. Boyer, S. Mazan, J. P. Bachellerie, A. Vignal, D. Cohen and M. Fontes. 'Relationship between Charcot-Marie-Tooth 1A and Smith-Magenis regions: snU3 may be a candidate gene for the Smith-Magenis syndrome.' Hum Mol Genet 2 (1993), pp. 1235-1243.

[37] T. B. Chou and N. Perrimon. 'Use of a Yeast Site-Specific Recombinase to Produce Female Germline Chimeras in Drosophila.' Genetics 131.3 (1992), pp. 643653.

[38] J. M. Crawford, N. Harden, T. Leung, L. Lim and D. P. Kiehart. 'Cellularization in Drosophila melanogaster is disrupted by the inhibition of rho activity and the activation of Cdc42 function.' Developmental Biology 204.1 (1998), pp. 151-164.

[39] S. De Renzis, O. Elemento, S. Tavazoie and E. F. Wieschaus. 'Unmasking activation of the zygotic genome using chromosomal deletions in the Drosophila embryo.' PLoS biology 5.5 (2007), e117.

[40] L. DeVeylder, T. Beeckman and D. Inzé. 'The ins and outs of the plant cell cycle.' Nature Reviews Molecular Cell Biology 8 (2007), pp. 655-665.

[41] E. J. Devor. 'Use of molecular beacons to verify that the serine hydroxymethyltransferase pseudogene SHMT-ps1 is unique to the order Primates.' Genome biology 2.2 (2001), pp. 0006.1-0006.5.

[42] S. Di Talia, R. She, S. A. Blythe, X. Lu, Q. F. Zhang and E. F. Wieschaus. 'Posttranslational control of Cdc25 degradation terminates drosophila's early cellcycle program.' Current Biology 23.2 (2013), pp. 127-132.

[43] C. Dinant and M. S. Luijsterburg. 'The emerging role of HP1 in the DNA damage response.' Molecular and cellular biology 29.24 (2009), pp. 6335-6340.

[44] B. A. Edgar and S. A. Datar. 'Zygotic degradation of two maternal Cdc25 mRNAs terminates Drosophila's early cell cycle program.' Genes. Dev. 10 (1996), pp. 1966-1977.

[45] B. A. Edgar, C. P. Kiehle and G. Schubiger. 'Cell cycle control by the nucleocytoplasmic ratio in early Drosophila development.' Cell 44 (1986), pp. 365-372. 
[46] B. A. Edgar, D. A. Lehman and P. H. O'Farrell. 'Transcriptional regulation of string (cdc25): a link between developmental programming and the cell cycle.' Development 120 (1994), pp. 3131-3143.

[47] B. A. Edgar and P. H. O'Farrell. 'Genetic control of cell division pattern in the Drososphila embryo.' Cell 57 (1989), pp. 177-187.

[48] B. A. Edgar and P. H. O'Farrell. 'The three postblastoderm cell cycles of Drosophila embryogenesis are regulated in G2 by string.' Cell 62 (1990), pp. 469480 .

[49] B. A. Edgar, F. Sprenger, R. J. Duronio, P. Leopold and P. H. O'Farrell. 'Distinct molecular mechanism regulate cell cycle timing at successive stages of Drosophila embryogenesis.' Genes. Dev. 8 (1994), pp. 440-452.

[50] J. C. Eissenberg and S. C. R. Elgin. 'HP1a: A Structural Chromosomal Protein Regulating Transcription.' Trends Genet. 30.3 (2014), pp. 103-110.

[51] S. H. Elsea, R. C. Juyal, S. Jiralerspong, B. M. Finucane, M. Pandolfo, F. Greenberg, A. Baldini, P. Stover and P. I. Patel. 'Haploinsufficiency of cytosolic serine hydroxymethyltransferase in the Smith-Magenis syndrome.' American journal of human genetics 57.6 (1995), pp. 1342-1350.

[52] M. S. Field, D. D. Anderson and P. J. Stover. 'Mthfs is an Essential Gene in Mice and a Component of the Purinosome.' Frontiers in genetics 2 (2011), pp. 1-13.

[53] M. S. Field, E. Kamynina, O. C. Agunloya, R. P. Liebenthal, S. G. Lamarre, M. E. Brosnan, J. T. Brosnan and P. J. Stover. 'Nuclear Enrichment of Folate Cofactors and Methylenetetrahydrofolate Dehydrogenase 1 (MTHFD1) Protect de Novo Thymidylate Biosynthesis during Folate Deficiency.' Journal of Biological Chemistry 289.43 (2014), pp. 29642-29650.

[54] V. E. Foe. 'Mitotic domains reveal early commitment of cells in Drosophila embryos.' Development 107 (1989), pp. 1-22.

[55] V. E. Foe and B. M. Alberts. 'Studies of nuclear and cytoplasmic behaviour during the five mitotic cycles that precede gastrulation in Drosophila embryogenesis.' Journal of cell science 61 (1983), pp. 31-70.

[56] P. Fogarty, R. F. Kalpin and W. Sullivan. 'The Drosophila maternal-effect mutation grapes causes a metaphase arrest at nuclear cycle 13.' Development (Cambridge, England) 120.8 (1994), pp. 2131-2142.

[57] P. Fogarty, S. D. Campbell, R. Abu-Shumays, B. de Saint Phalle, K. R. Yu, G. L. Uy, M. L. Goldberg and W. Sullivan. 'The Drosophila grapes gene is related to checkpoint gene chk1/rad27 and is required for late syncytial division fidelity.' Current biology 7.6 (1997), pp. 418-426. 
[58] J. T. Fox and P. J. Stover. 'Folate-mediated one-carbon metabolism.' In: Vitamins \& Hormones: Folic Acid and Folates. Vol. 79. London: Academic Press, 2008, pp. 1-44.

[59] J. T. Fox, W. K. Shin, M. a. Caudill and P. J. Stover. 'A UV-responsive internal ribosome entry site enhances serine hydroxymethyltransferase 1 expression for DNA damage repair'. Journal of Biological Chemistry 284.45 (2009), pp. 3109731108.

[60] M. Frasch, T. Hoey, C. Rushlow, H. Doyle and M. Levine. 'Characterization and localization of the even-skipped protein of Drosophila.' The EMBO journal 6.3 (1987), pp. 749-759.

[61] S. L. Fullilove and A. G. Jacobson. 'Nuclear elongation and cytokinesis in Drosophila montana.' Developmental Biology 26 (1971), pp. 560-577.

[62] T. A. Garrow, A. A. Brenner, V. M. Whitehead, X.-N. Chen, R. G. Duncan, J. R. Korenberg and B. Shane. 'Cloning of human cDNAs encoding mitochondrial and cytosolic serine hydroxymethyltransferases and chromosomal localization.' Journal of Biological Chemistry 268.16 (1993), pp. 11910-11916.

[63] E. Gateff. 'Malignant neoplasms of genetic origin in Drosophila melanogaster.' Science 200 (1978), pp. 1448-1459.

[64] D. M. Gilbert, S.-I. Takebayashi, T. Ryba, J. Lu, B. D. Pope, K. A. Wilson and I. Hiratani. 'Space and time in the nucleus developmental control of replication timing and chromosome architecture.' Cold Spring Harbor Symposia on Quantitative Biology 75 (2010), pp. 143-153.

[65] S. Girgis, I. M. Nasrallah, J. R. Suh, E. Oppenheim, K. A. Zanetti, M. G. Mastri and P. J. Stover. 'Molecular cloning, characterization and alternative splicing of the human cytoplasmic serine hydroxymethyltransferase gene.' Gene 210.2 (1998), pp. 315-324.

[66] S. Girgis, J. R. Suh, J. Jolivet and P. J. Stover. '5-Formyltetrahydrofolate Regulates Homocystein Remethylation in Human Neuroblastoma.' The Journal of biological chemistry 272.8 (1997), pp. 4729-4734.

[67] F. Greenberg, V. Guzzetta, R. Montes de Oca-Luna, R. E. Magenis, A. C. Smith, S. F. Richter, I. Kondo, W. B. Dobyns, P. I. Patel and J. R. Lupski. 'Molecular analysis of the Smith-Magenis syndrome: a possible contigu- ous-gene syndrome associated with del(17)(pll.2).' Am J Med Genet. 49 (1991), pp. 1207-1218.

[68] E. E. Grevengoed, D. T. Fox, J. Gates and M. Peifer. 'Balancing different types of actin polymerization at distinct sites: roles for Abelson kinase and Enabled.' Journal of Cell Biology 163 (2003), pp. 1267-1279.

[69] J. Großhans, H. J. Müller and E. Wieschaus. 'Control of cleavage cycles in Drosophila embryos by frühstart.' Developmental Cell 5.2 (2003), pp. 285-294. 
[70] J. Großhans, C. Wenzl, H.-M. Herz, S. Bartoszewski, F. Schnorrer, N. Vogt, H. Schwarz and H.-A. Müller. 'RhoGEF2 and the formin Dia control the formation of the furrow canal by directed actin assembly during Drosophila cellularisation.' Dev. Camb. Engl. 132 (2005), pp. 1009-1020.

[71] N. A. Haelterman, L. Jiang, Y. Li, V. Bayat, H. Sandoval, B. Ugur, K. L. Tan, K. Zhang, D. Bei, B. Xiong, W.-l. Charng, T. Busby, A. Jawaid, G. David, M. Jaiswal, K. J. T. Venken, S. Yamamoto, R. Chen and H. J. Bellen. 'Largescale identification of chemically induced mutations in Drosophila melanogaster.' Genome Research 24.10 (2014), pp. 1707-1718.

[72] K. L. Hari, A. Santerre, J. J. Sekelsky, K. S. McKim, J. B. Boyd and R. S. Hawley. 'The mei-41 gene of D. melanogaster is a structural and functional homolog of the human ataxia telangiectasia gene.' Cell 82.5 (1995), pp. 815-821.

[73] V. Hartenstein. Atlas of Drosophila development. New York: Cold Spring Harbor Laboratory Press, 1993.

[74] K. Herbig, E.-P. Chiang, L.-R. Lee, J. Hills, B. Shane and P. J. Stover. 'Cytoplasmic serine hydroxymethyltransferase mediates competition between folatedependent deoxyribonucleotide and S-adenosylmethionine biosyntheses.' Journal of Biological Chemistry 277.41 (2002), pp. 38381-38389.

[75] T.-a. Hori, D. Ayusawa, K. Shimizu, H. Koyama and T. Seno. 'Chromosome breakage induced by thymidylate stress in thymidylate synthase-negative mutants of mouse FM3A cells.' Cancer Research 44.2 (1984), pp. 703-709.

[76] C. Iampietro, J. Bergalet, X. Wang, N. A. L. Cody, A. Chin, F. A. Lefebvre, M. Douziech, H. M. Krause and E. Lécuyer. 'Developmentally regulated elimination of damaged nuclei involves a Chk2-dependent mechanism of mRNA nuclear retention.' Developmental Cell 29.4 (2014), pp. 468-481.

[77] T. Ishizaki, Y. Morishima, M. Okamoto, T. Furuyashiki and S. Narumiya. 'Coordination of microtubules and the actin cytoskeleton by the Rho effector mDia1.' Na. Cell. Biol. 3 (2001), pp. 8-14.

[78] D. A. Kane and C. B. Kimmel. 'The zebrafish midblastula transition.' Development 119 (1993), pp. 447-456.

[79] L.-R. Kao and T. Megraw. 'Centrocortin cooperates with centrosomin to organize Drosophila embryonic cleavage furrows.' Current Biology 19 (2009), pp. 937-942.

[80] E. K. Kastanos, Y. Y. Woldman and D. R. Appling. 'Role of mitochondrial and cytoplasmic serine hydroxymethyltransferase isozymes in de Novo purine synthesis in Saccharomyces cerevisiae.' Biochemistry 36.48 (1997), pp. 14956-14964.

[81] J. Knoblich. 'Epithelial polarity: The ins and outs of the fly epidermis.' Current Biology 10 (2000), R791-R794. 
[82] S. Kobayashi, K. Sato and Y. Hayashi. 'The Role fo Mitochondrial rRNAs and Nanos Protein in Germline Formation in Drosophila embryos'. Zoological Science 22 (2005), pp. 943-954.

[83] E. C. Kong, L. Allouche, P. A. Chapot, K. Vranizan, M. S. Moore, U. Heberlein and F. W. Wolf. 'Ethanol-regulated genes that contribute to ethanol sensitivity and rapid tolerance in drosophila.' Alcoholism: Clinical and Experimental Research 34.2 (2010), pp. 302-316.

[84] S. Kotadia, J. Crest, U. Tram, B. Riggs and W. Sullivan. 'Blastoderm Formation and Cellularisation in Drosophila melanogaster.' Encyclopedia of Life Sciences (2010), pp. 1-8.

[85] T. Kriegel and W. Schellenberger. 'Bioenergetik.' In: Biochemie und Pathobiochemie. Vol. 9. Berlin Heidelberg: Springer-Verlag, 2014, chapter 4.

[86] U. Landstrom, H. Lovtrup-Rein and S. Lovtrup. 'Control of cell division and cell differentiation by deoxynucleotides in the early embryo of Xenopus laevis.' Cell. Differ. 4 (1975), pp. 313-325.

[87] T. Lecuit, R. Samanta and E. Wieschaus. 'slam encodes a developmental regulator of polarized membrane growth during cleavage of the Drosophila ambryo.' Current Biology 23 (2002), pp. 2110-2120.

[88] T. Lecuit and E. Wieschaus. 'Polarized insertion of new membrane from a cytoplasmic reservoir during cleavage of the Drosophila embryo.' J. Cell. Biol. 150 (2000), pp. 849-860.

[89] M. Leptin. 'Gastrulatio in Drosophila: the logic and the cellular mechanisms.' EMBO Journal 18.12 (1999), pp. 3187-3192.

[90] H.-L. Liang, C.-Y. Nien, H.-Y. Liu, M. M. Metzstein, N. Kirov and C. Rushlow. 'The zinc-finger protein Zelda is a key activator of the early zygotic genome in Drosophila.' Nature 456.7220 (2008), pp. 400-403.

[91] U. Lim, K. Peng, B. Shane, P. J. Stover, A. A. Litonjua, S. T. Weiss, J. M. Gaziano, R. L. Strawderman, F. Raiszadeh, J. Selhub, K. L. Tucker and P. A. Cassano. 'Polymorphisms in cytoplasmic serine hydroxymethyltransferase and methylenetetrahydrofolate reductase affect the risk of cardiovascular disease in men.' The Journal of nutrition 135.8 (2005), pp. 1989-1994.

[92] H. D. Lindsay, D. J. Griffiths, R. J. Edwards, P. U. Christensen, J. M. Murray, F. Osman, N. Walworth and A. M. Carr. 'S-Phase-specific activation of Cds1 kinase defines a subpathway of the checkpoint response in Schizosaccharomyces pombe.' Genes 86 Development 12 (1998), pp. 382-395.

[93] X. Lu, J. Drocco and E. F. Wieschaus. 'Cell cycle regulation via inter-nuclear communication during the early embryonic development of Drosophila melanogaster.' Cell Cycle 9 (2010), pp. 2908-2910. 
[94] X. Lu, J. M. Li, O. Elemento, S. Tavazoie and E. F. Wieschaus. 'Coupling of zygotic transcription to mitotic control at the Drosophila mid-blastula transition.' Development 136 (2009), pp. 2101-2110.

[95] A. J. MacFarlane, X. Liu, C. A. Perry, P. Flodby, R. H. Allen, S. P. Stabler and P. J. Stover. 'Cytoplasmic serine hydroxymethyltransferase regulates the metabolic partitioning of methylenetetrahydrofolate but is not essential in mice.' Journal of Biological Chemistry 283.38 (2008), pp. 25846-25853.

[96] A. J. MacFarlane, C. A. Perry, M. F. McEntee, D. M. Lin and P. J. Stover. 'Shmt1 heterozygosity impairs folate-dependent thymidylate synthesis capacity and modifies risk of Apc(min)-mediated intestinal cancer risk'. Cancer Research 71.6 (2011), pp. 2098-2107.

[97] F. Martini, B. Maras, P. Tanci, S. Angelaccio, P. S., D. Barra, F. Bossa and V. Schirch. 'The primary structure of rabbit liver mitochondrial serine hydroxymethyltransferase.' Journal of Biological Chemistry 264.15 (1989), pp. 8509-8519.

[98] N. Masrouha, L. Yang, S. Hijal, S. Larochelle and B. Suter. 'The Drosophila chk2 gene loki is essential for embryonic DNA double-strand-break checkpoints induced in S phase or G2.' Genetics 163.3 (2003), pp. 973-982.

[99] I. Mavrakis, R. Rikhy and J. Lippincott-Schwartz. 'Plasma membrane polarity and compartmentalization are established before cellularization in the fly embryo.' Dev. Cell. 16 (2009), pp. 93-104.

[100] A. Mazumdar and M. Mazumdar. 'How one becomes many: blastoderm cellularization in Drosophila melanogaster.' BioEssays 24.11 (2002), pp. 1012-1022.

[101] S. Mazzalupo and L. Cooley. 'Illuminating the role of caspases during Drosophila oogenesis.' Cell death and differentiation 13.11 (2006), pp. 1950-1959.

[102] C. R. McClung, C. R. Davis, K. M. Page and S. A. Denome. 'Characterization of the formate (for) locus, which encodes the cytosolic serine hydroxymethyltransferase of Neurospora crassa.' Molecular and cellular biology 12.4 (1992), pp. 14121421.

[103] H. Murakami and H. Okayama. 'A kinase from fission yeast responsible for blocking mitosis in S phase.' Nature 374 (1995), pp. 817-819.

[104] A. Nakamura, R. Amikura, M. Mukai, S. Kobayashi and P. F. Lasko. 'Requirement for a noncoding RNA in Drosophila polar granules for germ cell establishment.' Science 274 (1996), pp. 2075-2079.

[105] J. W. Newport and M. W. Kirschner. 'Regulation of the cell cycle during early Xenpus development.' Cell 37 (1984), pp. 731-742.

[106] J. Newport and M. Kirschner. 'A major developmental transition in early Xenopus embryos: I. characterization and timing of cellular changes at the midblastula stage.' Cell 30 (1982), pp. 675-686. 
[107] J. Newport and M. Kirschner. 'A major developmental transition in early Xenopus embryos: II. Control of the onset of transcription.' Cell 30 (1982), pp. 687-696.

[108] C.-Y. Nien, H.-L. Liang, S. Butcher, Y. Sun, S. Fu, T. Gocha, N. Kirov, J. R. Manak and C. Rushlow. 'Temporal coordination of gene networks by Zelda in the early Drosophila embryo.' PLoS genetics 7.10 (2011), e1002339.

[109] E. A. Nigg and T. Stearns. 'The centrosome cycle: Centriole biogenesis, duplication and inherent asymmetries.' Nature Cell Biology 13.10 (2011), pp. 11541160 .

[110] G. Nishibuchi and J.-I. Nakayama. 'Biochemical and structural properties of heterochromatin protein 1: understanding its role in chromatin assembly.' Journal of biochemistry 156.1 (2014), pp. 11-20.

[111] K. A. Nyberg, R. J. Michelson, C. W. Putnam and T. A. Weinert. 'Toward maintaining the genome: DNA damage and replication checkpoints.' Annual review of genetics 36 (2002), pp. 617-656.

[112] P. H. O'Farrell, B. A. Edgar, D. Lakich and C. F. Lehner. 'Directing cell division during development.' Science 246 (1989), pp. 635-640.

[113] I. Oishi, S. Sugiyama, H. Otani, H. Yamamura, Y. Nishida and Y. Minami. 'A novel Drosophila nuclear protein serine/threonine kinase expressed in the germline during its establishment.' Mechanisms of Development 71.1-2 (1998), pp. 49-63.

[114] M. Padash Barmchi, S. Rogers and U. Häcker. 'DrhoGEF2 regulates actin organization and contractility in the Drosophila blastoderm embryo.' Journal of Cell Biology 168 (2005), pp. 575-585.

[115] A. F. Palazzo, T. A. Cook, A. S. Alberts and G. G. Gundersen. 'mDia mediates Rho-regulated formation and orientation of stable microtubules.' Nature Cell Biology 3 (2001), pp. 723-729.

[116] C. K. T. Pang, J. H. Huntera, R. Gujjara, R. Podutooria, J. Bowmana, D. G. Mudeppaa and P. K. Rathod. 'Catalytic and ligand-binding characteristics of Plasmodium falciparum serine hydroxymethyltransferase.' Mol. Biochem. Parasitol. 168 (2009), pp. 74-83.

[117] N. Perrimon. 'The maternal effect of l(1)discs-large: A recessive oncogene of Drosophila melanogaster.' Dev. Biol. 127 (1988), pp. 392-407.

[118] M. Peters, C. DeLuca, A. Hirao, V. Stambolic, J. Potter, L. Zhou, J. Liepa, B. Snow, S. Arya, J. Wong, D. Bouchard, R. Binari, A. S. Manoukian and T. W. Mak. 'Chk2 regulates irradiation-induced, p53-mediated apoptosis in Drosophila.' Proceedings of the National Academy of Sciences 99.17 (2002), pp. 11305-11310. 
[119] A. L. Piskac-Collier, C. Monroy, M. S. Lopez, A. Cortes, C. J. Etzel, A. J. Greisinger, M. R. Spitz and R. A. El-Zein. 'Variants in Folate Pathway Genes as Modulators of Genetic Instability and Lung Cancer Risk.' Genes, chromosomes \& cancer 50 (2011), pp. 1-12.

[120] O. Pourquie. 'Clocks regulating developmental processes.' Current Opinion in Neurobiology 8 (1998), pp. 665-670.

[121] J. Queiroz-Machado, J. Perigao, P. Simoes-Carvalho, S. Herrmann and C. E. Sunkel. 'tef: A mutation that causes telomere fusion and severe genome rearrangements in Drosophila melanogaster.' Chromosoma 110 (2001), pp. 10-23.

[122] J. Ren, X. Gao, C. Jin, M. Zhu, X. Wang, A. Shaw, L. Wen, X. Yao and Y. Xue. 'Systematic study of protein sumoylation: Development of a site-specific predictor of SUMOsp 2.0.' Proteomics 9 (2009), pp. 3409-3412.

[123] W. F. Rothwell and W. Sullivan. 'The centrosome in early Drosophila embryogenesis.' Current Topics in Developmental Biology 49 (2000), pp. 409-447.

[124] N. N. Rott and G. A. Sheeleva. 'Changes in the rate of cell divisions in the course of early development of diploid and haploid loach embryos.' J. Embryol. Exp. Morphol. 20 (1968), pp. 141-150.

[125] T. Rudolph, M. Yonezawa, S. Lein, K. Heidrich, S. Kubicek, C. Schäfer, S. Phalke, M. Walther, A. Schmidt, T. Jenuwein and G. Reuter. 'Heterochromatin Formation in Drosophila Is Initiated through Active Removal of H3K4 Methylation by the LSD1 Homolog SU(VAR)3-3.' Molecular Cell 26.1 (2007), pp. 103-115.

[126] P. Russell, S. Moreno and S. I. Reed. 'Conservation of mitotic controls in fission and budding yeasts.' Cell 57 (1989), pp. 295-303.

[127] P. Russell and P. Nurse. 'cdc25 + functions as an inducer in the mitotic control of fission yeast.' Cell 45 (1986), pp. 145-153.

[128] J. Sambrook and D. W. Russell. Molecular Cloning: A laboratory manual. 3rd ed. New York: Cold Spring Harbor Laboratory Press, 2001. ISBN: 978-0879695774.

[129] W. A. Samsonoff, J. Reston, M. McKee, B. O'Connor, J. Galivan, G. Maley and F. Maley. 'Intracellular location of thymidylate synthase and its state of phosphorylation.' J. Biol. Chem. 272 (1997), pp. 13281-13285.

[130] M. Sardiello, F. Licciulli, D. Catalano, M. Attimonelli and C. Caggese. 'MitoDrome: A database of Drosophila melanogaster nuclear genes encoding proteins targeted to the mitochondrion.' Nucleic Acids Research 31.1 (2003), pp. 322-324.

[131] V. Schirch and W. B. Strong. 'Interaction of folylpolyglutamates with enzymes in one-carbon metabolism.' Arch. Biochem. Biophys. 269 (1989), pp. 371-380.

[132] V. Schirch, S. Hopkins, E. Villar and S. Angelaccio. 'Serine hydroxymethyltransferase from Escherichia coli: purification and properties.' Journal of bacteriology 163.1 (1985), pp. 1-7. 
[133] F. Schweisguth, J. A. Lepesant and A. Vincent. 'The serendipity alpha gene encodes a membrane-associated protein required for the cellularization of the Drosophila embryo.' Genes and Development 4.6 (1990), pp. 922-931.

[134] F. Schweisguth, C. Yanicostas, F. Payre, J.-A. Lepesant and A. Vincent. 'Cisregulatory elements of the Drosophila blastoderm-specific serendipity alpha gene: Ectopic activation in the embryonic PNS is promoted by the deletion of an upstream element.' Dev. Biol. 136 (1989), pp. 181-193.

[135] A. W. Shermoen, M. L. McCleland and P. H. O'Farrell. 'Developmental control of late replication and s phase length.' Current Biology 20.23 (2010), pp. 2067-2077.

[136] O. C. M. Sibon, A. Laurençon, R. S. Hawley and W. E. Theurkauf. 'The Drosophila ATM homologue Mei-41 has an essential checkpoint function at the midblastula transition.' Current Biology 9.6 (1999), pp. 302-12.

[137] O. C. M. Sibon, V. A. Stevenson and W. E. Theurkauf. 'DNA-replication checkpoint control at the Drosophila midblastula transition.' Nature 388.6637 (1997), pp. 93-97.

[138] E. Silva, S. Tiong, M. Pedersen, E. Homola, A. Royou, B. Fasulo, G. Siriaco and S. D. Campvell. 'ATM is required for telomere maintenance and chromosome stability during Drosophila development.' Current Biology 14 (2004), pp. 13411347.

[139] S. Small and M. Levine. 'The initiation of pair-rule stripes in the Drosophila blastoderm.' Current opinion in genetics $\&$ development 1.2 (1991), pp. 255-260.

[140] A. Smith, L. McGavran, J. Robinson, G. Waldstein, J. Macfarlane, J. Zonona, J. Reiss, M. Lahr, L. Allen and E. Magenis. 'Interstitial deletion of (17)(p11.2p11.2) in nine patients.' Am J Med Genet. 3 (1986), pp. 393-414.

[141] K. Snell, U. Baumann, P. C. Byrne, K. J. Chave, S. B. Renwick, P. G. Sanders and S. K. Whitehouse. 'The genetic organization and protein crystallographic structure of human serine hydroxymethyltransferase'. Advances in Enzyme Regulation 40 (2000), pp. 353-403.

[142] Y.-H. Song, G. Mirey, M. Betson, D. A. Haber and J. Settleman. 'The Drosophila ATM Ortholog, dATM, Mediates the Response to Ionizing Radiation and to Spontaneous DNA Damage during Development'. Current Biology 14 (2004), pp. 1354-1359.

[143] J. Stein, H. Broihier, L. Moore and R. Lehmann. 'Slow as molasses is required for polarized membrane growth and germ cell migration in Drosophila.' Dev. Camb. Engl. 129 (2002), pp. 3925-3934.

[144] P. J. Stover. 'Physiology of folate and vitamin B12 in health and disease.' Nutrition reviews 62 (2004), S3-S13. 
[145] P. J. Stover, L. H. Chen, J. R. Suh, D. M. Stover, K. Keyomarsi and B. Shane. 'Molecular cloning, characterization and regulation of the human mitochondrial serine hydroxymethyltransferase gene.' Journal of Biological Chemistry 272.3 (1997), pp. 1842-1848.

[146] P. Stover and V. Schirch. '5-Formyltetrahydrofolate Polyglutamates Are Slow Tight Binding Inhibitors of Serine Hydroxymethyltransferase.' Journal of Biological Chemistry 266.3 (1991), pp. 1543-1550.

[147] U. Strausfeld, J. C. Labbe, D. Fesquet, J. C. Cavadore, A. Picard, K. Sadhu, R. P. and M. Dorée. 'Dephosphorylation and activation of a p34cdc2/cyclinB complex in vitro by human CDC25 protein.' Nature 351 (1991), pp. 242-245.

[148] W. B. Strong, S. J. Tendler, R. L. Seither, I. D. Goldman and V. Schirch. 'Purification and properties of serine hydroxymethyltransferase and C1-tetrahydrofolate synthase from L1210 cells.' J. Biol. Chem. 265 (1990), pp. 12149-12155.

[149] T. T. Su, S. D. Campbell and P. H. O'Farrell. 'grapes/CHK1 mutants are defective in cyclin proteolysis and coordination mitotic events.' Current biology 9.16 (1999), pp. 919-22.

[150] W. Sullivan, P. Fogarty and W. E. Theurkauf. 'Mutations affecting the cytoskeletal organization of syncytial Drosophila embryos.' Development 118.4 (1993), pp. 1245-1254.

[151] H.-W. Sung, S. Spangenberg, N. Vogt and J. Großhans. 'Number of nuclear divisions in the drosophila blastoderm controlled by onset of zygotic transcription.' Current Biology 23.2 (2013), pp. 133-138.

[152] D. M. E. Szebenyi, F. N. Musayev, M. L. Di Salvo, M. K. Safo and V. Schirch. 'Serine hydroxymethyltransferase: Role of Glu75 and evidence that serine is cleaved by a retroaldol mechanism.' Biochemistry 43.22 (2004), pp. 6865-876.

[153] S. Takada, A. Kelkar and W. E. Theurkauf. 'Drosophila checkpoint kinase 2 couples centrosome function and spindle assembly to genomic integrity.' Cell 113.1 (2003), pp. 87-99.

[154] S. Takada, S. Kwak, B. S. Koppetsch and W. E. Theurkauf. 'grp (chk1) replicationcheckpoint mutations and DNA damage trigger a Chk2-dependent block at the Drosophila midblastula transition.' Development 134.9 (2007), pp. 1737-1744.

[155] H. Takai, K. Tominaga, N. Motoyama, Y. A. Minamishima, H. Nagahama, T. Tsukiyama, K. Ikeda, K. Nakayama, M. Nakanishi and K. Nakayama. 'Aberrant cell cycle checkpoint function and early embryonic death in Chk1-/- mice.' Genes \&6 Development 14 (2000), pp. 1439-1447.

[156] J. R. ten Bosch, J. A. Benavides and T. W. Cline. 'The TAGteam DNA motif controls the timing of Drosophila pre-blastoderm transcription.' Development 133.10 (2006), pp. 1967-1977. 
[157] R. S. Tibbetts and R. T. Abraham. 'PI3K-related kinases: Roles in cell-cycle regulation andDNAdamage responses.' In: Signaling networks and cell cycle control: The molecular basis of cancer and other diseases. Ed. by J. Gutkind. Totowa, NJ: Humana Press, 2000, pp. 267-301.

[158] B. D. Towbin, C. González-Aguilera, R. Sack, D. Gaidatzis, V. Kalck, P. Meister, P. Askjaer and S. M. Gasser. 'Step-wise methylation of histone H3K9 positions heterochromatin at the nuclear periphery.' Cell 150.5 (2012), pp. 934-947.

[159] D. O. Trembecka-Lucas and J. W. Dobrucki. 'A heterochromatin protein 1 (HP1) dimer and a proliferating cell nuclear antigen (PCNA) protein interact in vivo and are parts of a multiprotein complex involved in DNA replication and DNA repair.' Cell Cycle 11.11 (2012), pp. 2170-2175.

[160] D. O. Trembecka-Lucas, A. T. Szczurek and J. W. Dobrucki. 'Dynamics of the HP1 $\beta$-PCNA-containing complexes in DNA replication and repair.' Nucleus 4.1 (2013), pp. 74-82.

[161] S. R. Turner, R. Ireland, C. L. Morgan and S. Rawsthorne. 'Identification and localization of multiple forms of serine hydroxymethyltransferase in pea (Pisum sativum) and characterization of a cDNA encoding a mitochondrial isoform.' $J$. Biol. Chem. 267 (1992), pp. 13528-13534.

[162] M. Uhlig. 'Lab-course Report.' Lab rotation. Insitute of Biochemistry, GeorgAugust-University Göttingen, 2014.

[163] L. Vastag, P. Jorgensen, L. Peshkin, R. Wei, J. Rabinowitz and M. W. Kirschner. 'Remodelling of the metabolome during early frog development.' PLOS 6 (2011), e16881.

[164] N. Vogt, I. Koch, H. Schwarz, F. Schnorrer and C. Nüsslein-Volhard. 'The gammaTuRC Components Grip75 and Grip128 Have an Essential Microtubule-Anchoring Function in the Drosophila Germline.' Development 133 (2006), pp. 39633972 .

[165] G. von Dassow and G. Schubiger. 'How an actin network might cause fountain streaming and nuclear migration in the syncytial Drosophila embryo.' Journal of Cell Biology 127 (1994), pp. 1637-1653.

[166] H. I. de Vries, L. Uyetake, W. Lemstra, J. F. Brunsting, T. T. Su, H. H. Kampinga and O. C. M. Sibon. 'Grp/DChk1 is required for G2-M checkpoint activation in Drosophila S2 cells, whereas Dmnk/DChk2 is dispensable.' Journal of cell science 118 (2005), pp. 1833-1842.

[167] Y. Wen, C. Eng, J. Schmoranzer, N. Cabrera-Poch, E. Morris, M. Chen, B. Wallar, A. Alberts and G. Gundersen. 'EB1 and APC bind to mDia to stabilize microtubules downstream of Rho and promote cell migration.' Nat. Cell. Biol. 6 (2004), pp. 820-830. 
[168] C. Wenzl, S. Yan, P. Laupsien and J. Großhans. 'Localization of RhoGEF2 during Drosophila cellularization is developmentally controlled by slam.' Mechanisms of Development 127.7-8 (2010), pp. 371-384.

[169] S. M. Wernimont, F. Raiszadeh, P. J. Stover, E. B. Rimm, D. J. Hunter, W. Tang and P. A. Cassano. 'Polymorphisms in serine hydroxymethyltransferase 1 and methylenetetrahydrofolate reductase interact to increase cardiovascular disease risk in humans.' The Journal of nutrition 141.2 (2011), pp. 255-260.

[170] K. Wilson. 'Preparation of genomic DNA from bacteria.' Current Protocols in Molecular Biology (1997), pp. 2.4.1-2.4.5. DOI: 10.1016/B978-0-12-4186873.00011-2.

[171] C. F. Woeller, D. D. Anderson, D. M. E. Szebenyi and P. J. Stover. 'Evidence for small ubiquitin-like modifier-dependent nuclear import of the thymidylate biosynthesis pathway.' Journal of Biological Chemistry 282.24 (2007), pp. 1762317631.

[172] L. Wolpert, C. Tickle, A. M. Arias, P. Lawrence, A. Lumsden, E. Robertson, E. Meyerowitz and J. Smith. Principles of Development. 5th ed. Oxford: Oxford University Press, 2015. ISBN: 978-0198709886.

[173] D. Woods and P. Bryant. 'Molecular cloning of the lethal (1) discs large-1 oncogene of Drosophila.' Dev. Biol. 134 (1989), pp. 222-235.

[174] D. Woods and P. Bryant. 'The discs-large tumor suppressor gene of Drosophila encodes a guanlyate kinase homolog localized at septate junctions.' Cell 66 (1991), pp. 451-464.

[175] D. Woods and P. Bryant. 'ZO-1, DlgA and PSD-95/SAP90: Homologous proteins in tight, septate, and synaptic cell junctions.' Mech. Dev. 44 (1993), pp. 85-89.

[176] D. Woods, C. Hough, D. Peel, G. Callaini and P. Bryant. 'Dlg protein is required for junction structure, cell polarity, and proliferation control in Drosophila epithelia.' J. Cell. Biol. 134 (1996), pp. 1469-1482.

[177] S. Yamamoto, M. Jaiswal, W.-l. Charng, T. Gambin, E. Karaca, G. Mirzaa, W. Wiszniewski, H. Sandoval, N. A. Haelterman, B. Xiong, K. Zhang, V. Bayat, G. David, T. Li, K. Chen, U. Gala, T. Harel, D. Pehlivan, S. Penney, M. Sivaci, E. Battaloglu, D. Muzny, Y.-w. Wan, Z. Liu, A. T. Lin-moore, R. Allikmets, R. A. Gibbs, R. Chen, J. R. Lupski and M. F. Wangler. 'A Drosophila Genetic Resource of Mutants to Study Mechanisms Underlying Human Genetic Diseases.' Cell 159.1 (2014), pp. 200-214.

[178] S. Yan, Z. Lv, M. Winterhoff, C. Wenzl, T. Zobel, J. Faix, S. Bogdan and J. Grosshans. 'The F-BAR protein Cip4/Toca-1 antagonizes the formin Diaphanous in membrane stabilization and compartmentalization.' J. Cell. Sci. 25 (2013), pp. $97-106$. 
[179] G. K. Yasuda and G. Schubiger. 'Temporal regulation in the early embryo: is MBT too good to be true?' Trends in Genetics 8 (1992), pp. 124-127.

[180] K. Yuan, A. W. Shermoen and P. H. O'Farrell. 'Illuminating DNA replication during Drosophila development using TALE-lights'. Current Biology 24.4 (2014).

[181] M. Zalokar and I. Erk. 'Division and migration of nuclei during early embryogenesis of Drosophila melanogaster.' Journal of Microbial Cell 25 (1976), pp. 97106.

[182] Q. Zhao, Y. Xie, Y. Zheng, S. Jiang, W. Liu, W. Mu, Y. Zhao, Y. Xue and J. Ren. 'GPS-SUMO: a tool for the prediction of sumoylation sites and SUMO-interaction motis.' Nucleic Acid Research 41 (2014), W325-W330.

[183] Z. Zhao, C. C. Lee, S. Jiralerspong, R. C. Juyal, F. Lu, A. Baldini, F. Greenberg, C. T. Caskey and P. I. Patel. 'The gene for a human microfibril-associated glycoprotein is commonly deleted in Smith-Magenis syndrome patients.' Hum Mol Genet 4 (1995), pp. 589-597.

[184] X. Zhu, L. M. Stevens and D. Stein. 'Synthesis of the sulfate donor PAPS in either the Drosophila germline or somatic follicle cells can support embryonic dorsal-ventral axis formation.' Development 134.8 (2007), pp. 1465-1469.

[185] L. Zou and S. J. Elledge. 'Sensing DNA damage through ATRIP Recognition of RPA-ssDNA Complexes.' Science 300 (2003), pp. 1542-1548. 


\section{Part II}

Fluctuation analysis of centrosomes reveals a cortical function of Kinesin-1 


\section{Introduction}

\subsection{The cytoskeleton in early Drosophila embryos}

Early development of Drosophila melanogaster takes place in a syncytium. The nuclei divide without an accompanied cytokinesis. The nuclei, which resemble cellular units, lay in a common cytoplasm and are not separated by cell membranes. In syncytial blastoderm stage these nuclei are anchored beneath the cortex and form a single layer in the embryo 62. This makes the syncytial Drosophila embryo a good model system to analyse the dynamics of the cytoskeleton. The cytoskeleton in the embryo is not restricted to small cells. It forms a potentially large network, which extends over the whole embryo simplifying manipulations of the system $[14,36]$.

A pair of centrosomes is located above each nucleus (Figure 62A). The centrosomes are microtubule organising centres and associate with their (-)-ends. Microtubule asters emanate from the centrosomes and overlap with asters from neighbouring nuclei. The nuclei of the syncytial embryo behave synchronously and undergo cell-cycle-specific changes and movements, which result in cycles of ordered and unordered nuclear arrangements [21]. Microtubules and F-actin, a second component of the cytoskeleton also perform cell-cycle-specific changes in their arrangement and localisation (Figure 2) [22]. In interphase F-actin is accumulated at the cortex and forms cap-like structures, the actin caps. During prophase and metaphase of mitosis, plasma membranes invaginate between the nuclei to a depth a appr. $8 \mu \mathrm{m}$. These plasma membranes are called pseudocleavage or metaphase furrows, since they persist only temporally and are regressed at the end of mitosis. Plasma membrane invagination and regression is accompanied by redistribution of F-actin. During mitosis F-actin becomes localised at the leading edge of the invaginating furrow and relocalises to actin caps at the end mitosis [44]. During cellularisation F-actin is localised at the tip of the invaginating furrow, the furrow canal, similar to previous cycles [15]. Microtubules switch between an aster-like structure in interphase and a spindle structure in mitosis (Figure 62A). During cellularisation, microtubule asters form an inverted basket-like structure around the nuclei and microtubules are elongated throughout the cellularisation process and are important for furrow invagination. The cellularisation furrow invaginates to a depth of appr. $35 \mu \mathrm{m}$, where it contracts laterally to enclose the nuclei into cells. Slam is a membrane associated protein localised to the tip of the invaginating, which is required for initiation of furrow invagination as well as for polarity establishment $[1,25,41]$. 
In interphase, cortical F-actin and microtubules depend on each other. Actin caps are induced by centrosomes and the Scrambled protein in an microtubule-independent manner [34, 42], and F-actin anchors microtubules to the cortex. This cortical anchoring is necessary to position and separate the centrosome pairs. Centrosomes duplicate in early interphase, at the beginning of mitosis before nuclear envelope breakdown they start to move to the equator of their associated nucleus (Figure 62A) [14]. The centrosome movement is depending on the molecular motors Dynein and Myo-II as well as F-actin polymerisation by the actin nucleators Arp2/3 and Dia [9, 35, 39]. A recent publication investigated the principles, which underly the rapid dispersal of nuclei in the syncytial Drosophila embryo, since an even distribution of nuclei and their anchorage to the embryonic cortex is required for proper development [46]. In a cell-free system, they studied nuclear separation in predefined volumes over multiple nuclear division cycles. They could show that mitotic spindle, microtubule asters, and F-actin cooperate also in isolated cytoplasm lacking membrane structures to define a characteristic separation length scale, which seems to be a conserved property in early development of Drosophila.

\subsection{Components of the cytoskeleton}

The syncytial stage of early Drosophila development is characterised by a network behaviour, which is reflected by dynamic changes in morphology from interphase to mitosis. These changes are for example redistribution of the cortex and the ordered and unordered arrangement of nuclei, which is often visible as a "mitotic wave" moving from the poles to the middle of the embryo and is completed by large-scale drift movements of nuclei and cytoplasm and the end of mitosis [16, 21]. It seems that centrosomes and their associated microtubule asters can act collectively to generate stresses resulting in movements of the nuclei. These movements are driven by active elements of the cytoskeleton, which will be introduced in detail in the following.

\subsubsection{Actin}

Actin is one of the most abundant proteins in eukaryotic cells [32]. Globular actin (Gactin) can self-assemble to filamentous actin (F-actin) [24]. F-actin is a helical doublestranded polymer and can assemble to different structures such as linear bundles, twodimensional network structures as well s three-dimensional gel-like structures [11]. Since the spontaneous polymerisation of actin is very slow and thermodynamically unfavourable, it is supported and regulated by actin-accessory proteins [40]. Beside catalysing actin polymerisation, actin-accessory proteins have multiple functions such as to maintain the actin monomer pool, to control the length of actin filaments, to regulate polymerisation and depolymerisation of actin filaments and to control and perform cross-linking 


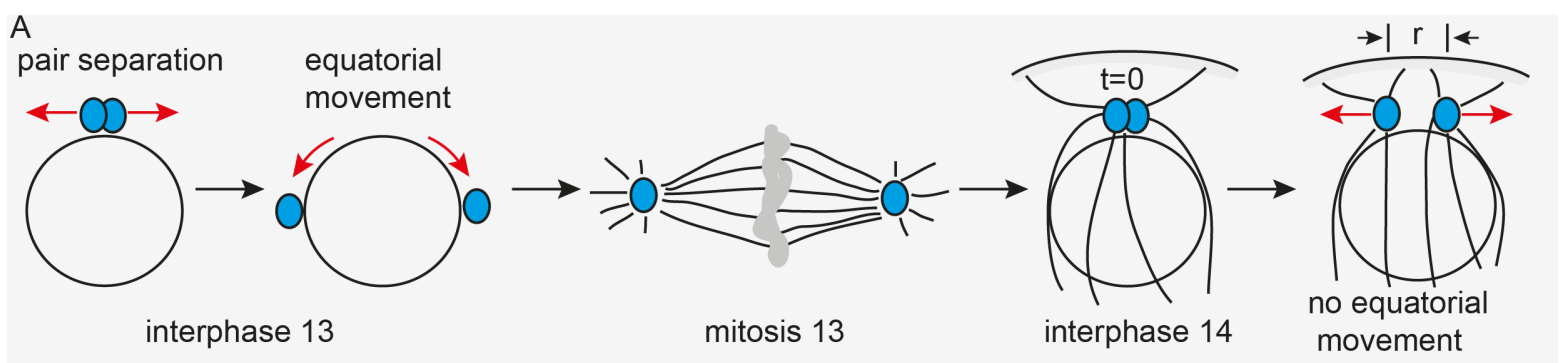

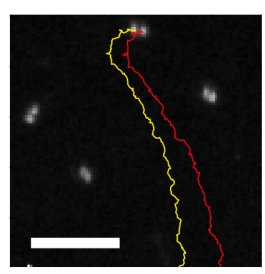

C

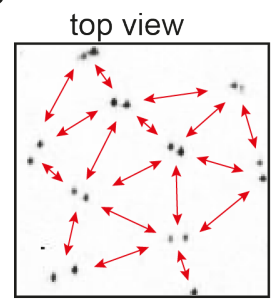

lateral interaction
D

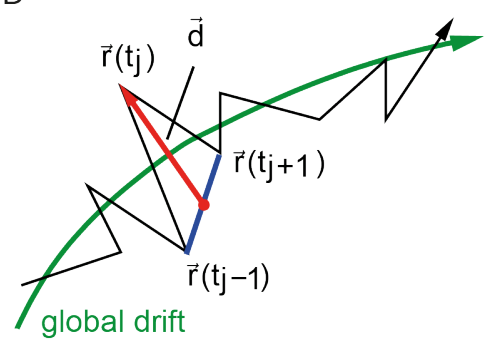

Figure 62: Schematic representation of centrosome cycle and its lateral and cortical interactions. A: Schematic drawing of centrosome cycle. Centrosome, marked in blue, separate in early interphase. Before nuclear envelope breakdown the centrosome separate and move to opposing positions equatorial to the nucleus. In interphase, however, this equatorial movement does not happen. Centrosome split and separate to a short distance. B: Traces, shown in red and yellow, of a single centrosome pair over two minutes. The generation of tracing was the first step of the fluctuation analysis procedure. C: Schematic drawing of lateral and cortical interactions. Lateral (left image) and cortical interaction (right image) determine the mobility of centrosome pairs and their associated nuclei. Centrosomes are shown in black, nuclei in grey and interactions are indicated as red arrows. D: Definition of the fluctuation parameter D. 
of actin-filaments to actin networks. Functions of F-actin and actin-accessory are mostly ruled out by studying mutant systems and upon affecting its function with antagonistic drugs. Latrunculin A is an inhibitor of actin polymerisation. Injection of this drug into cellularising embryos causes loss of cellularisation furrows indicating that the plasma membrane integrity is dependent on continuous actin polymerisation at the furrow [8]. Engulfment and Cell Motility (ELMO) is an important protein to establish actin caps in interphase, since elmo mutants have a uniform cortical distribution of F-actin lacking actin caps [26]. The mutant embryos do not form metaphase furrows frequently resulting in nuclei with chromosome segregation defects. These nuclei are removed from the cortex and fall into the interior of the embryo. Beside this, the embryos pass through syncytial cell cycles with a similar timing as WT embryos. The mutation becomes lethal at cellularisation stage, since no cellularisation furrow is formed and gastrulation can not be initiated properly. Drosophila embryos carrying mutations in the arp2/3 gene, which encodes an actin nucleator, exhibit a disrupted metaphase furrow formation and show a defective actin cap expansion at the beginning of mitosis inidicating that Arp $2 / 3$ is, similar to ELMO, important for actin cap formation and redistribution of F-actin into metaphase and cellularisation furrows. However, in the absence of Arp2/3 actin caps are formed, indicating the involvement of further actin nucleators and / or elongators in this process [43, 47]. Sponge is non-canonical Rac-GEF, which is also required for the formation of actin caps and metaphase furrows [3, 33]. Embryos carrying mutations in the sponge gene show actin caps of reduced size compared to WT embryos.

\subsubsection{Microtubules and centrosomes}

Microtubules are cytoskeletal components, which are important for the initiation of furrow invagination. Disruption of microtubules in the slow phase of cellularisation abolishes invagination, whereas its disrupion at the beginning of fast phase has no effect on furrow invagination [13]. Microtubules are organised by and emanate from centrosomes, which are built of a pair of centrioles surrounded by a pericentriolar matrix (PCM). Both centrioles and the PCM are associated with various proteins regulating their function and replication. Replication of centrosomes is tightly regulated and over- or underreplication is associated with various diseases, including cancer [30]. Three proteins are known so far to be required for replication of the centrioles: Sas-6, Sas-4 and Sak. Especially Sas-6 is important to prevent overreplication of centrioles, since overexpression of GFP-Sas-6 induces extra rounds of centriole replication in various tissues of Drosophila [30]. Centrosomes are conserved organelles, which exhibit a dramatical change in their function and structure in cell cycle progression and cell differentiation. In mitosis they are essential to determine the cell division axis. Furthermore, they are necessary for cilia formation during interphases [2]. Centrosome positioning in a cell is actively maintained. Both microtubules and actomyosin are important for their positioning. Microtubules exert pushing and pulling forces involving microtubule dynamics and micotubule motor 
proteins such as Dynein, a process which is contributed by actomyosin contractility [7, 37]. Recent studies in Drosophila embryos indicated that Myosin II is not required for centrosome separation prior to nuclear envelope breakdown, but that it requires actin rearrangements at the growing edge of the actin caps. These rearrangements are driven by both Arp2/3 and Formin proteins [8]. Furthermore, Cao et al. could show that the mechanisms, which separate centrosomes after nuclear envelope breakdown, do not depend on actin remodelling. They concluded that the actin-dependent centrosome separation has an important implication for the positioning of centrosomes in various processes such as cell migration, maintenance of cell polarity and asymmetric cell division (please refer to [8] and references therein).

\subsubsection{Microtubule motor proteins}

The cytoskeletal network is characterised by dynamic changes, which are induced upon force generating active elements. Dynein, Kinesins and actin-based Myosins are such active elements in the cytoskeleton. Furthermore polymerisation of actin filaments, enhanced by elongation factors (e.g. Dia) or nucleators (e.g. Arp2/3) can also generate forces. These dynamic changes compensate for cell-cycle specific rearrangements of cellular components to establish a regular spatial organisation of the nuclei, which is required for proper development. In the following specific active elements are introduced.

Kinesin-1 is a $(+)$-end directed microtubule motor protein [18, 23], which transports determinants for patterning of the embryonic axes [5, 19]. Furthermore it participates in axonal transport and in the distribution of nuclei in syncytial muscles [28, 31]. Kinesin-1 depleted embryos exhibit a normal syncytial development, indicating that Kinesin- 1 is not essential for mitosis in contrast to Kinesin-5 [45]. However, Kinesin-1 is required for cellularisation. It is loaded onto microtubules by Ensconsin [28, 45]. Embryos carrying mutations in the ensconsin gene show a similar phenotype as kinesin-1 mutants indicating that the phenotype is due to the missing function of Kinesin- 1 on microtubules rather than a function of Ensconsin independent from Kinesin-1. Kinesin-1 is known to transport Dynein to the posterior cortex, where both proteins colocalise and are assumed to anchor posterior determinants and germ plasm [5, 19]. Dynein is a (-)-end directed microtubule motor protein [18, 23]. It accumulates at actin caps and is implied to contribute to formation and function of the mitotic spindle [12]. Depleting the maternal contribution of Dynein heavy chain results not only in mitotic defects during the syncytial cycles of Drosophila melanogaster, but also in disrupted cellularisation and gastrulation indicating further functions in these developmental processes [35]. 


\subsection{Aim of the work}

Microtubule asters of neighbouring asters overlap and interact with each other to establish a regular spatial organisation of the nuclei (Figure 62B). Furthermore, the interaction of microtubule asters with actin caps and cortical actin is required for this organisation. The functional interaction between these has been analysed in detail, mainly in a static manner. Network behaviour in a cell with a time-scale of seconds has been little investigated so far. Therefore, we investigated centrosomal movements, which were recorded at $1 \mathrm{~Hz}$ and performed analysis of pair separation and individual movement (Figure 62C and D). The dynamics of centrosomes serve as a proxy for the movement of microtubule asters, since they are organised by and emanate from centrosomes. This may provide further information about the organisation of the microtubule network and interaction of individual microtubule asters with each other, with specific microtubule motor proteins and with the cortex. We wanted to identify, which forces and stresses drive the nuclear rearrangements and the cytoplasmic flow. With this study we want to improve the understanding of how cells mechanically interact and function collectively to form a specific tissue.

\subsection{Contribution to the project}

This project was carried out as a collaborative work. Prof. Dr. Jörg Großhans designed the experimental approach. I performed the biological experiments and imaging of centrosomes and nuclei including of all WT and mutant embryos and injection procedures, except the imaging of azide injected and fixed embryos, which was carried out by Dr. Maheshwar Gummalla (Institute for Developmental Biochemistry, Medical School, Georg-August-University Göttingen), as well as immunostaining experiments and time-lapse recordings of non Sas6-GFP or Sas4-GFP expressing embryos. Dr. Timo Aspelmeier (Institute of Mathematical Stochastics, Georg-August-University Göttingen) conceived the methods for quantitative data analysis. Image analysis was done by Dr. Timo Aspelmeier and Lutz Künneke (Institute for Theoretical Physics, Georg-AugustUniversity Göttingen).

The manuscript, which was accepted for publication by the journal "Biophysical Journal", is attached in the following section. 



\title{
Fluctuation Analysis of Centrosomes Reveals a Cortical Function of Kinesin-1
}

\author{
Franziska Winkler, ${ }^{1}$ Maheshwar Gummalla, ${ }^{1}$ Lutz Künneke, ${ }^{2}$ Zhiyi Lv, ${ }^{1}$ Annette Zippelius, ${ }^{2}$ Timo Aspelmeier, ${ }^{3,4}$ \\ and Jörg Grosshans ${ }^{1, *}$ \\ ${ }^{1}$ Institute for Developmental Biochemistry, Medical School, ${ }^{2}$ Institute for Theoretical Physics, ${ }^{3}$ Institute for Mathematical Stochastics, and \\ ${ }^{4}$ Felix Bernstein Institute for Statistics in the Biosciences, Georg-August-University Göttingen, Göttingen, Germany
}

ABSTRACT The actin and microtubule networks form the dynamic cytoskeleton. Network dynamics is driven by molecular motors applying force onto the networks and the interactions between the networks. Here we assay the dynamics of centrosomes in the scale of seconds as a proxy for the movement of microtubule asters. With this assay we want to detect the role of specific motors and of network interaction. During interphase of syncytial embryos of Drosophila, cortical actin and the microtubule network depend on each other. Centrosomes induce cortical actin to form caps, whereas F-actin anchors microtubules to the cortex. In addition, lateral interactions between microtubule asters are assumed to be important for regular spatial organization of the syncytial embryo. The functional interaction between the microtubule asters and cortical actin has been largely analyzed in a static manner, so far. We recorded the movement of centrosomes at $1 \mathrm{~Hz}$ and analyzed their fluctuations for two processes-pair separation and individual movement. We found that F-actin is required for directional movements during initial centrosome pair separation, because separation proceeds in a diffusive manner in latrunculin-injected embryos. For assaying individual movement, we established a fluctuation parameter as the deviation from temporally and spatially slowly varying drift movements. By analysis of mutant and drug-injected embryos, we found that the fluctuations were suppressed by both cortical actin and microtubules. Surprisingly, the microtubule motor Kinesin-1 also suppressed fluctuations to a similar degree as F-actin. Kinesin-1 may mediate linkage of the microtubule $(+)$-ends to the actin cortex. Consistent with this model is our finding that Kinesin-1-GFP accumulates at the cortical actin caps.

\section{INTRODUCTION}

The syncytial embryo of Drosophila is a system well suited to the investigation of cytoskeletal networks and functional interactions between microtubule asters and actin cortex. As no cell membranes separate the cellular units, the cytoskeleton forms a potentially large network extending over the whole embryo $(1,2)$. Microtubule asters with a pair of centrosomes in their center overlap with neighboring asters, thus forming a network. Each microtubule aster is associated with a nucleus. Network behavior is reflected by nuclear dynamics in syncytial embryos. The nuclei form an array that undergoes cell-cycle-dependent movements and cycles between unordered and ordered arrangements (3). Nuclear dynamics is associated with fast stereotypic changes of the cytoskeleton. Within minutes, microtubules switch between spindles in mitosis and asters in interphase, whereas F-actin switches from cortical caps in interphase to furrows in mitosis. Microtubules and cortical actin interact functionally. Besides being

Submitted October 14, 2014, and accepted for publication July 31, 2015. *Correspondence: jgrossh@gwdg.de

This is an open access article under the CC BY-NC-ND license (http:// creativecommons.org/licenses/by-nc-nd/4.0/)

Franziska Winkler, Maheshwar Gummalla, and Lutz Künneke contributed equally to this work.

Editor: Margaret Gardel.

(C) 2015 The Authors

0006-3495/15/09/0856/13 organizers of the microtubule asters, centrosomes induce the formation of cortical actin caps (4), whereas F-actin is required for cortical anchoring of the microtubule network. During interphase, the cortical link is important for positioning and separation of centrosome pairs. After duplication in early interphase, the two daughter centrosomes move apart. Before nuclear envelope breakdown, they move to the equator of the associated nucleus (1). The equatorial movement depends on actin polymerization by Dia and Arp2/3 as well as the molecular motors Myosin-II and Dynein (5-7). It is unknown, however, whether and how the actin cortex contributes to initial separation of centrosome pairs and to dynamics of centrosomes and microtubule asters in interphase.

Active cytoskeletal networks in cells are far away from the thermodynamic equilibrium. Despite this, the cytoskeleton acquires stable steady-state structures on a timescale longer than minutes. In contrast, at short timescales in the millisecond range, thermal diffusion may dominate. In the intermediate range of seconds, fluctuations of the network may reflect the active nonequilibrium dynamics of the cytoskeleton (8). Molecular motors are a potential driving force for the dynamics of cytoskeletal networks. Although filament dynamics has been well characterized, the behavior of networks within a cell on a timescale of seconds, to our 
knowledge, has been little investigated. Thus, analysis of trajectories and fluctuations of centrosomes may provide information about microtubules network organization and interactions between individual asters and with the actin cortex, which goes beyond information obtained from static images.

Kinesin- 1 is the prototype of the $(+)$-end directed microtubule motors (9). In Drosophila, Kinesin-1 is required for transport of determinants for patterning of the embryonic axes $(10,11)$, axonal transport, and distribution of nuclei in syncytial muscle $(12,13)$. Kinesin-1 acts together with Ensconsin (ens), which loads Kinesin-1 onto microtubules $(12,14)$. A function of Kinesin-1 in syncytial embryos has not been investigated, yet.

Here, we establish fluctuation analysis of centrosome dynamics to reveal mechanisms driving the initial separation of centrosome pairs and centrosome movement in interphase. With these analyses and by applying a mechanical model, we found that F-actin provides directed forces for initial pair separation in contrast to separation by random interactions. By fluctuation analysis of centrosomes in interphase, we revealed that cortical actin caps and, surprisingly, the microtubule motor Kinesin-1 as well, suppress centrosome fluctuations. Based on this and additional genetic data, we propose a model in that Kinesin-1 mediates linkage of the microtubule network to the actin cortex to stabilize the microtubule network and the associated nuclear array.

\section{MATERIALS AND METHODS}

\section{Drosophila genetics}

The following mutations and fly strains were used: FRT[G13], $K h c^{27}$ (14), $e^{\text {ens }}{ }^{\text {swo }}$ (12), Dmn-GFP (Dynamitin) (15), Dlc-GFP (Dynein light chain), Kinesin-1-GFP (14), Sas6-GFP (16), Sas4-GFP (16), and ced-12/ELMO ${ }^{367}$ (EMS mutant isolated in a screen for blastoderm mutations in germline clones (17). MTD-Gal4 (maternal GAL4) (18), Kinesin-1 RNA (PTRiP. $_{\mathrm{i}}$ GL00330attP2; Transgenic $\mathrm{RNA}_{\mathrm{i}}$ Resource Project, Harvard Medical School, Boston, MA), Utrophin-GFP (19), and Zipper-GFP (zip ${ }^{\text {CC01626, }}$ Bloomington Drosophila Stock Center, Indiana University, Bloomington, IN). Kinesin- 1 depleted embryos were obtained from females carrying one copy of each of the following: the Kinesin- $1 \mathrm{RNA}_{\mathrm{i}}$, the MTD-Gal4, and either Sas4-GFP, Dlc-GFP, or Dmn-GFP transgenes. Kinesin-1 depleted embryos were recognized by their cellularization defect. Germline clones were generated by FRT/Flipase-mediated mitotic recombination and selection with ovo ${ }^{D}$. The $E L M O^{367}$ lethality and blastoderm phenotypes were mapped by meiotic recombination to the $b$ region. The lethality was fine-mapped to a $43 \mathrm{~kb}$ region by noncomplementation with molecularly defined deficiencies available from the Bloomington Drosophila Stock Center. ELMO ${ }^{367}$ is an allele of $E L M O$ as it did not complement the c06760 allele, whereas mutations of the other genes in the region were complemented.

\section{Molecular genetics}

The Ubiquitin promoter (PstI-BglII) (20), Drosophila dynein light chain (SacII-BamHI) (21), and eGFP (BamHI-BamHI-KpnI) were inserted between the PstI-KpnI sites of a pUASt derivative lacking the UAS and hsp70TATA sites. PCR cloning was verified by sequencing of all fragments.

\section{Histology}

Embryos were fixed by $4 \%$ formaldehyde (37\% for microtubules staining) and stained according to standard procedures (22). F-actin was detected with labeled phalloidin (Invitrogen, Carlsbad, CA) and DAPI $(0.2 \mu \mathrm{g} / \mathrm{mL})$. The following antibodies were employed: monoclonal anti- $\alpha$-Tubulin (Sigma-Aldrich, St. Louis, MO) and goat IgGs coupled with Alexa dyes (at final $4 \mu \mathrm{g} / \mathrm{mL}$, Invitrogen).

\section{Microinjection}

Dechorionated embryos were aligned on a coverslip, briefly desiccated, and covered with halocarbon oil (Voltalef 10S; Lehmann \& Voss, Hamburg, Germany). Colcemid (100 mg/mL; CalbioChem, San Diego, CA), Latrunculin A (100 mg/mL; Cayman Chemical, Ann Arbor, MI), Jasplakinolide (1 mM), or Y-27632 (10 mM, Sigma-Aldrich) were injected into Sas6GFP-expressing embryos. Time-lapse recording was started immediately after injection. Slam dsRNA was synthesized and injected as described previously in Wenzl et al. (22). Latrunculin A, colcemid, and Y-27632 activities were verified by injection into embryos expressing UtrophinGFP, Tubulin-GFP, and Zipper-GFP, respectively. Jasplakinolide activity was verified by fluorescence-recovery-after-photobleach analysis in Utrophin-GFP-expressing embryos. Injection of latrunculin A before onset of anaphase induced a nuclear fallout phenotype with approximately onehalf of the nuclei and their centrosomes falling into the interior of the embryo. After colcemid injection, the centrosomes were not able to separate from each other in the following mitosis. Sodium azide $(0.02 \%$ in water) was injected in Sas4-GFP-expressing embryos in mitosis interphase 13. Recording was started 3 min after injection.

\section{Microscopy}

Time-lapse recording was performed with an inverted microscope with a spinning disk unit (model No. CSU-X1; Carl Zeiss, Jena, Germany) and an AxioCam MRm camera (Carl Zeiss). The temperature was $20-23^{\circ} \mathrm{C}$. Seven to nine sections covering $\sim 2 \mu \mathrm{m}$ were recorded with a EC Plan NeoFluar objective $(40 \times /$ NA1.3/oil; Carl Zeiss $)$ at a frame rate of $1 \mathrm{~Hz}$ for up to $20 \mathrm{~min}$ with an exposure time of $30 \mathrm{~ms}$. The stack size of $2 \mu \mathrm{m}$ corresponds to $\sim 2.5 \%$ of the embryonic radius. Thus, perspective distortions at the edges were $<2.5 \%$, allowing us to treat the images as two-dimensional. For fast imaging at $20 \mathrm{~Hz}$, single planes were recorded for 5 min with a PlanApochromat lens $(100 \times /$ NA1.4/oil; Carl Zeiss $)$ and an electronmultiplying charge-coupled device camera (Evolve 512; Photometrics, Huntington Beach, CA). A $20-\mathrm{Hz}$ recording was started shortly after start of centrosome duplication, when centrosomes stopped moving in the apical-basal direction. The $C Z I$ files with the original data were exported into omeTIFF files, and imported into the software IMAGEJ (National Institutes of Health, Bethesda, MD) as 16-bit TIFF stacks. The $Z$ sections were fused using the GROUPED $Z$ project option of IMAGEJ. Fixed embryos and movies of Kinesin-1-GFP, Dlc-GFP, and Dmn-GFP were recorded with a confocal microscope (model No. LSM780, Plan Apochromat lens, $63 \times$, NA1.4, oil; Carl Zeiss). For low illumination conditions, the laser intensity was reduced to one-third of the normal intensity.

\section{Image analysis and tracking}

Each image, in a time series of fluorescence microscopic images taken of an embryo, consists of a (typically nonzero) background signal and a number of bright spots, each of which is created by a fluorescence-labeled centrosome. Due to the diffraction limit of the optical microscope, these spots are not pointlike but blurred by a point-spread function $h$ over a region of a diameter of $\sim 300 \mathrm{~nm}$ in the image, corresponding to several pixels. Furthermore, the image is noisy due to the fact that the fluorophores emit photons in a statistical manner. This leads to Poisson noise in the image, 
and one of the consequences of this is the fact that the relative strength of the noise increases with decreasing light intensity, which limits the frame rate (i.e., the exposure time per frame) of the movies. We hence have the image generation model

$$
I_{t}(x) \sim \operatorname{Poisson}\left(\left(h \times f_{t}\right)(x)+b_{t}(x)\right),
$$

where $x$ is the location of an image pixel, $I_{t}(x)$ is the measured signal at time $t$, pixel $x, f_{t}$ is the density of centrosomes at time $t$, and $b_{t}$ is the background signal at $t$. The asterisk symbol (*) is the convolution operator.

To be able to identify the individual centrosomes reliably even when the intensity is low or when a centrosome is poorly lit due to a statistical fluctuation, it is necessary to employ a method that takes the image generation process and noise characteristics into account. Therefore, we adapted a maximum likelihood approach. In this approach, one tries to find the $\widehat{f}_{t}, \widehat{b}_{t}$ configuration of centrosomes that maximizes the conditional probability $P\left(I_{t} \mid \widehat{f}_{t}, b_{t}\right)$ of obtaining the actually observed image, i.e.,

$$
\left(\widehat{f}_{t}, \widehat{b}_{t}\right)=\arg \min _{\left(f^{\prime}, b^{\prime}\right)}\left(-\log \left(P\left(I_{t} \mid f^{\prime}, b^{\prime}\right)\right)\right) .
$$

This method alone, however, is not sufficient, as there are two additional difficulties. First, the blurring of the image by the microscope destroys information on small length scales (smaller than the size of the spots). This is especially important when the centrosomes are close to each other, which is the case just after centrosome doubling. In particular, without further help, the method cannot decide whether there are one, two, or more centrosomes hidden under a spot. Second, there is the background signal, which complicates centrosome detection because a naive implementation of the above scheme would attribute a local signal to either the background signal or the centrosome density (or both); however, we would have no way of knowing which is correct. Incidentally, the background signal also makes centrosome identification by other methods (such as thresholding) difficult because it varies from movie to movie, from frame to frame, and also within each frame. For centrosome detection, the background must therefore be included in the image analysis.

The difficulties can be surmounted by supplying additional information to the method in the form of sparsity constraints. From the knowledge of the system being observed it is clear that the density of centrosomes is sparse, i.e., the number of centrosomes in each image is much less than the number of pixels in each image. It is also clear that the background signal is very smooth, i.e., slowly varying in space (in contrast to the signal caused by the centrosomes, which is strongly peaked). This smoothness of the background signal can be modeled by sparsity of the spatial Fourier transform of $b$ (23). Hence our method of centrosome detection consists of the maximum likelihood method, augmented by sparsity enforcing terms, so our estimate of the centrosome density (and the background signal) is

$$
\begin{aligned}
\left(\widehat{f}_{t}, \widehat{b}_{t}\right)= & \arg \min _{f^{\prime}, b^{\prime}}\left(-\log \left(P\left(I_{t} \mid f^{\prime}, b^{\prime}\right)\right)\right. \\
& \left.+\lambda_{1}\left\|f^{\prime}\right\|_{1}+\lambda_{2}\left\|\tilde{b}^{\prime}\right\|_{1}\right)
\end{aligned}
$$

with the additional constraint that $f^{\prime}(x), b^{\prime}(x) \geq 0$ for all $x$. Here, the 1-norm is defined by $\left\|f^{\prime}\right\|_{1}=\sum_{x}\left|f^{\prime}(x)\right|$ and analogously for $\tilde{b}^{\prime}$, the Fourier transform of $b^{\prime}$. The constants $\lambda_{1}, \lambda_{2}>0$ are suitably chosen parameters, which depend, among other things, on the background intensity, its variation, or the signal strength. In this work, we determined the values empirically. Automatic data-driven parameter selection would also be feasible, e.g., by cross-validation (24) or by statistical multiscale methods (25), but this was not the focus of this article. Approaches related to the one presented here have been suggested in the context of superresolution microscopy (26-28).

The terms involving the 1-norms have the effect of forcing most entries of $f^{\prime}$ and $\tilde{b}^{\prime}$ to 0 . They are convex functionals, such that this procedure amounts to a convex optimization problem, for which efficient numerical methods exist. We chose the alternating direction of multipliers method (29), which works well for this case.

When the density $\widehat{f}$, is found by minimizing Eq. 1 , we extract a list of centrosome positions from the nonzero entries of $f_{t}$. The centrosomes in successive frames are then matched to each other by a proximity criterion, thus forming individual time tracks. When centrosome doubling occurs, this is recognized by the fact that a new track starts in midmovie, and its partner is determined as the closest centrosome at the time of the appearance of the track. Because centrosome identification can sometimes fail despite all efforts, we also employ corrections for the cases when centrosomes are missing in individual frames.

In some of our figures, the data are plotted as a function of the time after splitting. This refers to the time elapsed since a centrosome was last seen to duplicate. Each centrosome is hence assigned its individual time by setting its internal clock to 0 at the time of the last split. In this way, we can aggregate information from different times and different positions on the embryo, but which has a functional correspondence.

\section{Fluctuations}

In Fig. $1 A$, a typical time track of a centrosome pair is shown. Clearly, the motion of the centrosomes undergoes some sort of smooth directed motion with nonsmooth fluctuations about it. Our interest here is not the drift motion but rather the fluctuations.

Often, the mean-square displacement is used for analysis in particle tracking (30). As the smooth part of the motion is, in our case, very irregular (it varies strongly between centrosomes and it sometimes reverses direction, for example), we found it convenient to quantify the fluctuations directly by differencing, namely by considering the quantity (see Fig. 2 A) (31),

$$
\begin{aligned}
& D_{i}\left(t_{j}\right)=\frac{\left(\vec{d}_{i}\left(t_{j}\right)\right)^{2}}{\Delta t} \text { with } \\
& \vec{d}_{i}\left(t_{j}\right)=\vec{r}_{i}\left(t_{j}\right)-\frac{1}{2}\left(\vec{r}_{i}\left(t_{j-1}\right)+\vec{r}_{i}\left(t_{j+1}\right)\right),
\end{aligned}
$$

where $\vec{r}_{i}(t)$ is the trajectory of centrosome $i$ and $t_{1}, t_{2}, \ldots$ are the times at which the frames were taken. We assume that the frames were recorded equidistantly such that $t_{i+1}-t_{i}=\Delta t$ with the same $\Delta t$ for all frames.

This quantity has the advantage that any smooth drift movement is removed to a very good approximation by taking the difference between the position of a centrosome at an intermediate time $t_{j}$, and the average position of the adjacent times $t_{j-1}$ and $t_{j+1}$, if the time increment $\Delta t$ is small enough such that the drift does not change appreciably in that interval.

To illustrate this behavior, we consider the simple case that $\vec{r}_{i}(t)$ undergoes Brownian motion in a flow field $\vec{v}(\vec{r}, t)$. Dropping the particle index $i$, this can be described by the Langevin equation

$$
\frac{d \vec{r}}{d t}=\vec{v}(\vec{r}, t)+\gamma \vec{\xi}(t),
$$

where $\vec{\xi}$ is Gaussian random noise with average $\mathbb{E}(\vec{\xi}(t))=0$ and $\mathbb{E}\left(\xi_{\alpha}(t)\right.$ $\left.\xi_{\beta}\left(t^{\prime}\right)\right)=\delta_{\alpha \beta} \delta\left(t-t^{\prime}\right)$ (here and in the following, the symbol $\mathbb{E}$ denotes the average), and $\gamma>0$ is the noise strength. For a spatially and temporally constant flow field $\vec{v}(\vec{r}, t)=\vec{v}_{0}=$ const., the motion over a time interval $\Delta t$ is given by $\vec{r}\left(t_{j+1}\right)=\vec{r}\left(t_{j}\right)+\vec{v}_{0} \Delta t+\vec{b}\left(t_{j}\right)$, where the increment $\vec{b}$ is a Gaussian random vector with covariance $\gamma^{2} \Delta t I_{2}$, with $I_{2}$ being the $2 \times 2$ identity matrix. Increments at different times $t_{j}, t_{j+1}, t_{j+2}, \ldots$ are independent from each other. Inserting this into the expression for $\vec{d}_{i}\left(t_{j}\right)$ shows that the flow vector $\vec{v}_{0}$ drops out completely and only the increments remain. Taking the average over the Gaussian distributions of the increments then yields the diffusion constant

$$
\mathbb{E} D_{i}\left(t_{j}\right)=\gamma^{2},
$$


A

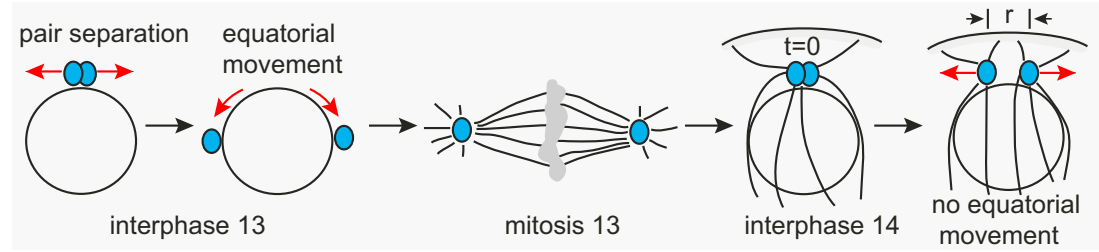

B

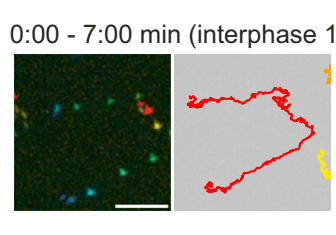

E

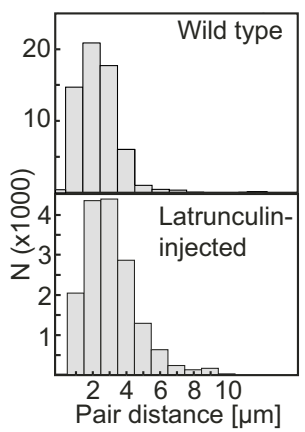

$\mathbf{F}$

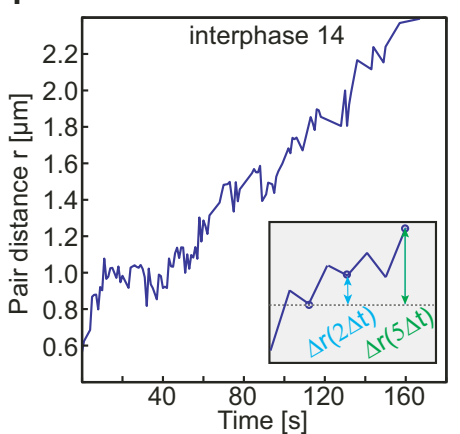

C Interphase 14

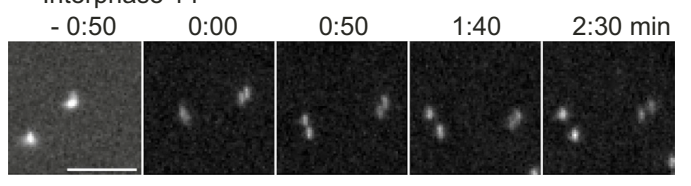

G

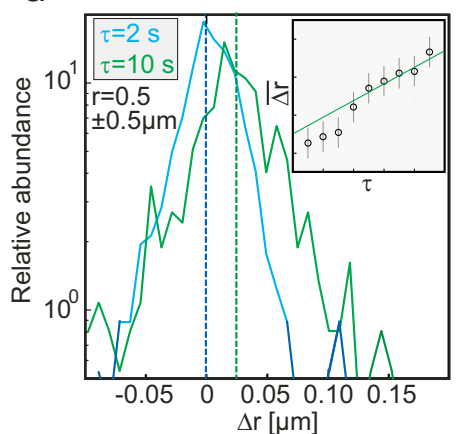

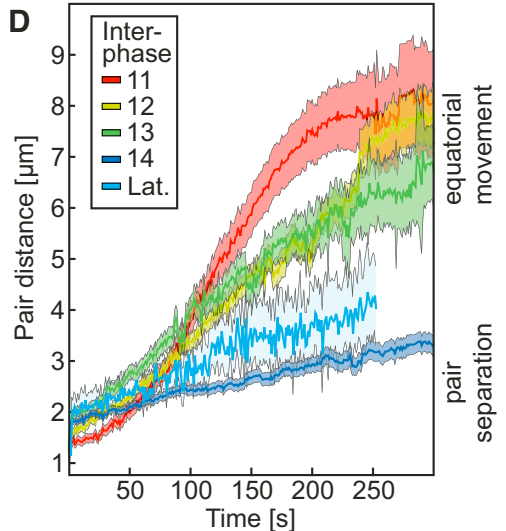

H Directional force-driven movement

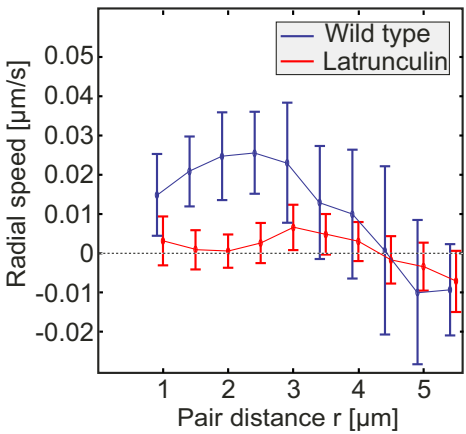

FIGURE 1 Directional F-actin-dependent movement during initial centrosome separation. (A) Schematic drawing of centrosome (blue) cycle. Spindle pole position in mitosis, showing position between plasma membrane and nucleus during interphase. Duplication of centrosome in early interphase and pair separation before mitosis. (Red arrows) Centrosome movement. (B) Trajectory of a centrosome pair (red). (Color-coding from red to blue) Direction of path between 0:00 and 7:00 min. Scale bar $=5 \mu \mathrm{m}$. (C) Images from movie of centrosomes marked with SAS-6-GFP. Scale bar $=5 \mu \mathrm{m}$. $(D)$ Pair distance (average, standard deviation) of centrosome pairs during interphases 1-14 and latrunculin-injected embryos in cycle 14. (E) Histograms of pair distances for interphase 14 in wild-type and latrunculin-injected embryos. (F) Representative trace of a single centrosome pair. (Inset) Illustration of the definition of the pair-distance changes $\Delta r$ as a function of elapsed time $\tau$. (G) Distributions of pair-distance changes $\Delta r$ for the indicated range of initial pair distances and elapsed times $\tau$. (Dashed lines) Respective averages of $\overline{\Delta r}$. (Inset) $\overline{\Delta r}$ varies linearly with $\tau$. Exemplary data set. (H) Average radial speed dependent upon pair distance for centrosomes in wild-type (blue) and latrunculin-injected (red) embryos.

independent of $\Delta t$. Of course, in our experiments we cannot assume that the motion is truly Brownian (see, e.g., Lau et al. (32) and Wessel et al. (33)), hence we prefer the term "fluctuations" for the quantity $D_{i}\left(t_{j}\right)$ rather than "diffusion constant". However, the ability of the fluctuation parameter $D$ to separate fast random fluctuations from slowly changing drift movement is not limited to this simplified Langevin model but remains valid in more complicated cases, provided there is a sufficient separation of timescales between the two.

In practice, there is always a measurement error associated with the position measurements $\vec{r}_{i}(t)$ such that $\vec{r}_{i}^{\prime}(t)=\vec{r}_{i}(t)+\sigma \vec{\epsilon}_{i}(t)$ is observed, where $\sigma$ is the magnitude of the error and the $\vec{\epsilon}_{i}(t)$ can be approximated by independent Gaussian random vectors with covariance $I^{2}$ (but see Savin and Doyle $(34,35)$ for a detailed discussion of possible measurement errors and their implications). Inserting $\vec{r}_{i}^{\prime}$ in place of $\vec{r}_{i}$ in Eq. 2 yields the observed

$$
\vec{d}_{i}^{\prime}\left(t_{j}\right)=d_{i}(t)+\sigma \vec{\epsilon}_{i}\left(t_{j}\right)-\frac{\sigma}{2}\left(\vec{\epsilon}_{i}\left(t_{j-1}\right)+\vec{\epsilon}_{i}\left(t_{j+1}\right)\right)
$$

The $E$ values can be combined into one effective random vector $\vec{\eta}_{i}\left(t_{j}\right)=\vec{\epsilon}_{i}\left(t_{j}\right)-(1 / 2)\left(\vec{\epsilon}_{i}\left(t_{j-1}\right)+\vec{\epsilon}_{i}\left(t_{j+1}\right)\right)$, which is a Gaussian random vector with covariance $(3 / 2) I_{2}$. The observed fluctuations $D^{\prime}{ }_{i}\left(t_{j}\right)$ hence acquire an additional noise contribution, $D_{i}^{\prime}\left(t_{j}\right)=\left(\left(\vec{d}_{i}\left(t_{j}\right)+\sigma \vec{\eta}_{i}\left(t_{j}\right)\right)^{2}\right) / \Delta t$. Denoting the average over the measurement error by an overbar, we obtain

$$
\overline{D_{i}^{\prime}\left(t_{j}\right)}=D_{i}\left(t_{j}\right)+3 \frac{\sigma^{2}}{\Delta t}
$$

Hence a nonzero measurement error $\sigma$ makes the observed fluctuation $D_{i}\left(t_{j}\right)$ diverge as $\Delta t$ goes to zero. In practice, therefore, we need to seek an appropriate $\Delta t$, which is small enough for $D_{i}\left(t_{j}\right)$ to be insensitive to the drift and large enough to be insensitive to measurement error. This issue is addressed in the Results.

For testing whether the mean value of the fluctuations for two different treatments can be considered to be equal, we used Welch's $t$-test. Under the null hypothesis that the means are equal, the observed data would have had a probability $p$ of occurring, where $p$ is the respective $p$-value listed in Table S3. The MATLAB function $t$-test2 was used for the actual calculations.

\section{Simulation of fluctuations}

To distinguish the qualitative behavior of the fluctuations for different types of movements, we performed simulations on a simplified model system of $n$ pointlike objects in the plane. There are no interactions between particles but each particle performs a random (Brownian) movement plus a drift. Because the particles do not interact, we focus on one particular particle. That particle's position $\vec{r}(t)$ is then described by Eq. 3 . In addition to the 


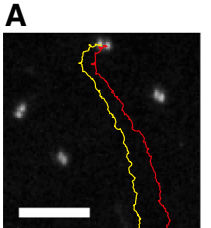

B

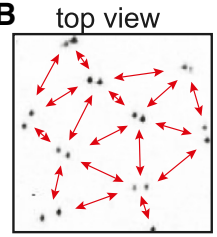

lateral interaction

D

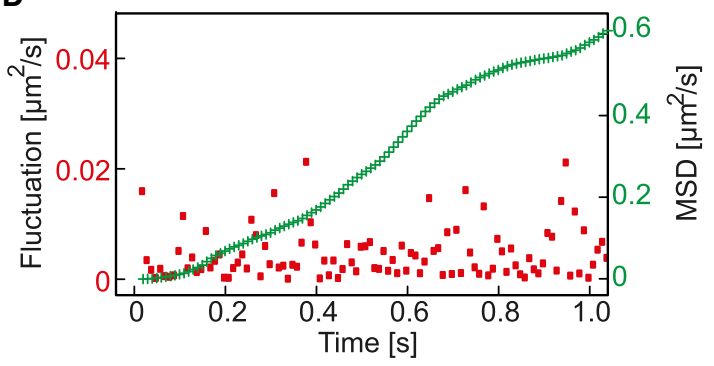

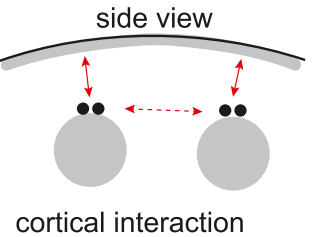

cortical interaction

H

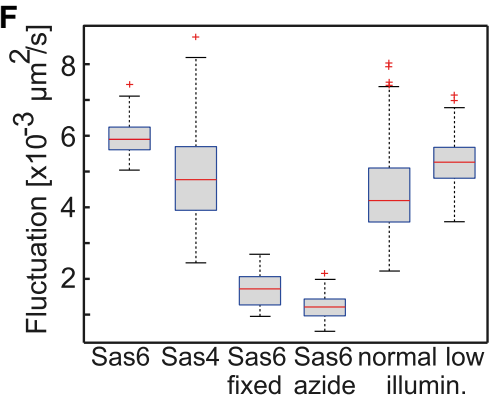

C

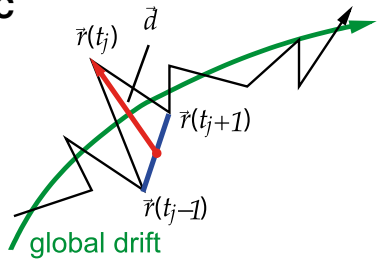

G

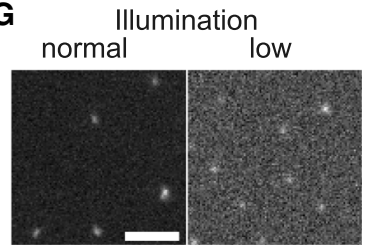

E

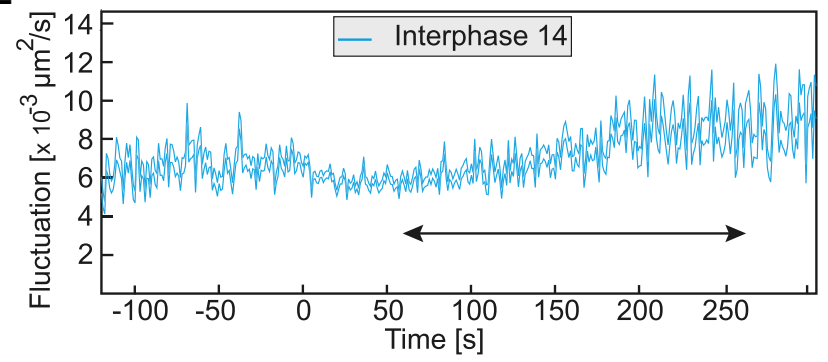

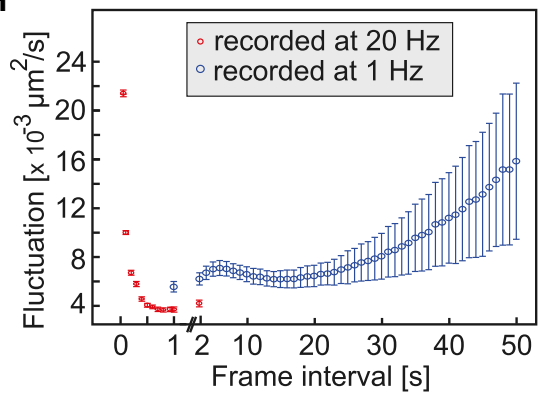

I

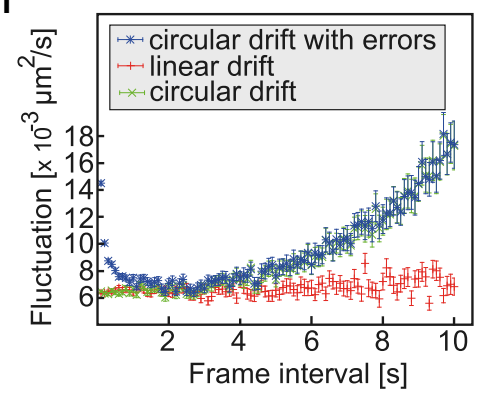

FIGURE 2 Fluctuation parameters. (A) Traces (yellow and red) of a centrosome pair over two minutes. (B) Lateral and cortical interactions (arrows in red) determine mobility of centrosome pairs (black disks) and their associated nuclei ( gray disk). $(C)$ Definition of the fluctuation parameter $D$. ( $D$ ) Comparison of MSD ( green) and fluctuation parameter ( red). As the simulations are based on manually chosen parameters, the resulting absolute numbers may differ from the experiments. $(E)$ Averaged time course of $D$ (range of standard deviation). The value $t=0$ represents time of centrosome splitting. (Arrow in black) Averaged period (60-260 s). ( $F$ ) Time-averaged fluctuation (60 s $<t<260 \mathrm{~s})$. Sas4-GFP and Sas6-GFP are two centrosome markers. ( $F$ and $G)$ Images with normal and low (one-third of laser intensity) illumination. $(H)$ Dependence of $D$ (average and standard deviation) on the frame interval for data sets recorded at a frame rate of $20 \mathrm{~Hz}$ (red) and $1 \mathrm{~Hz}$ (blue). (I) Dependence of $D$ on the frame interval calculated with computed data sets containing fluctuations with linear drift (red), fluctuations with circular drift (green), and fluctuations with circular drift and measurement error $($ blue $)$. Scale bars $=5 \mu \mathrm{m}$.

stochastic forces $\vec{\xi}$, we also allow for measurement errors as in Fluctuations, described above, by recording $\vec{r}^{\prime}(t)=\vec{r}(t)+\sigma \vec{\epsilon}(t)$, i.e., a noisy version of the true position $\vec{r}(t)$.

For the flow field $\vec{v}(\vec{r}, t)$, we chose two variants. The first is a constant linear drift, $\vec{v}(\vec{r}, t)=\vec{v}_{0}$. According to our construction of the fluctuation parameter $D$, such a linear drift will have no influence on the measurements of $D$, irrespective of $\Delta t$. The second variant is a circular flow field, $\vec{v}(\vec{r}, t)=c \vec{r}^{\perp}(\vec{r})$, where $c>0$ is a constant and $\vec{r}^{\perp}(\vec{r})$ is defined for $\vec{r}=(x, y)^{T}$ by $\vec{r}^{\perp}(\vec{r})=(-y, x)^{T}$. For the simulations shown below, we used the values $\gamma=0.08 \mu \mathrm{m} / \sqrt{\mathrm{s}}, \vec{v}_{0}=(-0.04,0.04)^{T} \mu \mathrm{m} / \mathrm{s}$, and $c=0.04 / \mathrm{s}$. The values for $\vec{v}_{0}$ and $c$ were chosen such that the magnitude of the velocity field roughly agrees with estimates of typical drift velocities in experiments.

From a simulation of these equations, we obtained a time series of simulated measured particle positions $\vec{r}(t)$ (corresponding to $\sigma=0$ ) and its noisy version $\vec{r} \prime(t)$ (corresponding to $\sigma>0$ ), and then calculated the noiseless fluctuation parameter $D(t)$ and its noisy counterpart $D^{\prime}(t)$ for the simulated particle for different values of $\Delta t$. For each $\Delta t$, these values were averaged over $t$ to obtain a $D$ as a function of $\Delta t$, as shown in Fig. $2 I$.

In the linear drift case, and with $\sigma=0$, there are no measurement errors, only fluctuations (this type of behavior is marked as linear drift in Fig. 2 I). As expected, the fluctuation parameter in this case does not depend on $\Delta t$ because the assumption of a spatially and temporally constant drift is satisfied. The measured value agrees with the expected value of $\gamma^{2}=$ $0.0064 \mu \mathrm{m}^{2} / \mathrm{s}$.

In the case of the circular drift, and with $\sigma=0$, the fluctuations $D$ coincide with the case of the linear drift for small $\Delta t<3 \mathrm{~s}$, as expected (circular drift in Fig. $2 I$ ). In the opposite case, the time interval $\Delta t$ is so large that the assumption of homogeneous drift breaks down and the measured values of $D$ deviate from the ideal value.

When measurement errors are switched on for the circular drift (we chose $\sigma=0.016 \mu \mathrm{m}$, which is approximately the size of a pixel in the experiments), the values of $D$ also start to deviate noticeably from the ideal value for small $\Delta t<1 \mathrm{~s}$ (circular drift with errors in Fig. 2 I). However, in the intermediate regime between 1 and $3 \mathrm{~s}$, the estimation of $D$ works reliably.

\section{Radial speed: a qualitative measure for centrosomal forces}

When we consider the process of separation of the centrosome pairs, we observe the following behavior. Each pair of centrosomes has a time-dependent distance (Fig. $1 C$ ). We locate all events in space and time where a pair has an intrapair distance within a small interval around some $r_{0}$ and then make a histogram of their distance a certain time $\tau$ later. Such a histogram is shown in Fig. $1 \mathrm{G}$. We observe that the width of this histogram increases 
with $\tau$, which is a sign of a diffusionlike process. In addition, the location of the average of the histogram moves toward greater distances. This is the signature of a force-driven process: without a force acting, there would be no change in the average pair distance. However, this is only strictly true for short times $\tau$ because for longer times the distribution of the distance would reach the minimal value of 0 where additional effects become relevant. Hence, we can interpret the radial speed $v_{r_{0}}$ defined by the speed of the average of the intrapair distance after an initial separation of $r_{0}$ as a measure of the force acting at a distance of $r_{0}$. In a purely viscous system with Gaussian random forces, this analogy would be exact (36). Here, the situation is more complex because the cytosol is a viscoelastic medium and a quantitative determination of the force would require knowledge of the complete viscoelastic response function (see Levine and Lubensky (37) for the response function of a model system).

Note that it is not clear whether the force is a repelling force acting between the centrosomes or an attractive force acting from the outside and pulling the centrosomes apart. Here, we merely use the intrapair distance as a measure for the distance of the centrosomes from their midpoint, i.e., the center of their nucleus. This allows us to circumvent the problem of the global drift movement, which we do not know.

Assuming a Gaussian approximation to the probability density $p_{r_{0}}(r, \tau)$ of the separation $r$ after time $\tau$, given a separation $r_{0}$ at time 0 ,

$$
p_{r_{0}}(r, \tau)=\frac{1}{\sqrt{2 \pi \tau} s} \exp \left(-\frac{\left(r-r_{0}-v_{r_{0}} \tau\right)^{2}}{2 s^{2} \tau}\right),
$$

with the radial speed $v_{r_{0}}$ and the diffusion constant $s^{2}$, we see that this is the solution of a diffusion equation

$$
\frac{\partial}{\partial \tau} p_{r_{0}}=-v_{r_{0}} \frac{\partial}{\partial r} p+\frac{1}{2} s^{2}\left(\frac{\partial}{\partial r}\right)^{2} p
$$

This equation is what one would expect for particles subject to a random force (the second term) plus a deterministic force (the first term). It can indeed be derived from a more fundamental Langevin equation and its corresponding Fokker-Planck equation but, as mentioned above, only for a purely viscous system. Nevertheless, it shows again the qualitative connection between the radial speed $v_{r_{0}}$ and the deterministic force acting at distance $r_{0}$.

The assumption of a Gaussian distribution is only justified for small $\tau$ when the particles did not have time to move far. In our experiments, we are limited to larger values of $\tau$, and we observe deviations from the Gaussian behavior (see Fig. $1 G$ ). However, qualitatively, the argument that a force induces a temporal increase of the average separation remains intact.

\section{RESULTS}

\section{Analysis of centrosomal mobility and fluctuations}

To assay microtubule network dynamics in vivo, we established a quantitative assay for recording and analyzing centrosome movement at a temporal resolution of one image per second in syncytial Drosophila embryos. We imaged centrosomes, which are associated with the (-)-ends and form the microtubule organizing centers. Representing the nodes of the microtubule network, centrosomes dynamics may serve as a proxy for the microtubule network dynamics. The centrosomes were genetically labeled by GFP-tagged components of the centrioles (Sas4-GFP, Sas6-GFP).

We first determined the trajectories of centrosomes by image segmentation and tracking (see Image Analysis and
Tracking, above). For detection, we developed a maximum likelihood approach, taking into account the complete image generation process and additional information such as sparsity constraints, which are known a priori about the system. This approach has the benefit that it is nonheuristic and can naturally deal with background signals, which are spatially and temporally slowly varying, thus avoiding user interaction.

At the end of mitosis, the centrosomes move from the spindle poles to an apical position between the cortex and the newly formed daughter nuclei (Fig. $1 A$ and Movies $\mathrm{S} 1$ and S2). Soon after this movement, duplication leads to a pair of centrosomes (Fig. $1 A-C$ ). In our assays, we define the time of duplication as $t=0$. During interphase, the centrosome pair separates and the centrosomes subsequently move to opposing equatorial positions in the nucleus before the next mitosis (Fig. 1, $A$ and $B$ ). The equatorial movement has been analyzed previously by slow timelapse recording and shown to be dependent on F-actin $(5,38)$. The mechanisms determining the movement during initial pair separation have not been analyzed.

We first extracted a time series of pair distances $r$ from our movies (Fig. 1, $D$ and $E$ ). The minimal pair distance that we can detect is in the range of $0.5 \mu \mathrm{m}$. The average pair distance increased first slowly and then rapidly before mitosis and nuclear envelope breakdown, as described previously in Cao et al. (5). The pair distance at the end of each interphase, except interphase 14, corresponds to the equatorial distance. This distance decreases with each interphase, as the nuclei become smaller. In interphase 14, centrosome pairs persist, as mitosis 14 occurs only $1 \mathrm{~h}$ later in development. As initial pair separation and equatorial movement are two distinct processes, we focus in the following only on cycle 14, which does not include an equatorial movement.

\section{Role of F-actin in initial separation of centrosome pairs}

We regard motion of the centrosome pairs as a superposition of directed and diffusionlike movement. We applied a statistical analysis of the fluctuations to separate these two components. The approach and its justification is explained in Radial Speed: A Qualitative Measure for Centrosomal Forces. By analyzing the time series of individual pair distances, fluctuations became obvious (Fig. $1 F$ ). We divided the pair distances $r$ into bins of $0.5-\mu \mathrm{m}$ width and collected all pair distance changes $\Delta r$, which happened within each bin after a time $\tau$ had passed (Fig. $1 F$, inset) and plotted the distribution of $\Delta r$ for each bin (Fig. $1 G$ ). This was done for a range of $\tau$-values. We observed that the averages of these distributions (Fig. $1 G$, dotted vertical lines) increased with $\tau$ as a function of the initial pair distance. For each initial pair distance, we hence estimated the radial speed by applying a linear fit to the average pair distance as a function of $\tau$ (Fig. $1 \mathrm{G}$, inset). 
The result is shown in Fig. $1 \mathrm{H}$. In a simplified model, in which the centrosomes are considered to be spherical particles moving in a purely viscous medium and subject to both Gaussian random forces and a distance-dependent deterministic force, the average speed is, in fact, up to a constant factor equal to the force (see also Sainis et al. (36)). In our case, the situation is more complicated, as the medium is viscoelastic. In principle, the complete response function of the medium needs to be known to calculate the forces from the radial speeds (see, e.g., Levine and Lubensky $(37,39)$ ). (For a theoretical description, see MacKintosh and Schmidt (40), Weihs et al. (41), and Wirtz (42); and for an overview, see Radial Speed: A Qualitative Measure for Centrosomal Forces.) In addition, the medium is inhomogeneous, containing organelles of the same length scale as the centrosomes, which makes a continuum description difficult. Nevertheless, our measurements indicate that up to a distance of 3-4 $\mu \mathrm{m}$, the pairs separate systematically, whereas centrosomes move independently with no significant radial speed for larger distances. These findings suggest a corresponding range of pair separation forces of $\sim 2-3 \mu \mathrm{m}$. Due to the caveat that the medium is not purely viscous and inhomogeneous, Fig. $1 \mathrm{H}$ is a qualitative measure of the force.

Centrosome movement is driven by microtubules and F-actin $(5,6,38)$. Previously, it has been shown that centrosome movement to the nuclear equator before nuclear envelope breakdown in mitosis depends on both microtubules and F-actin (5). We tested the contribution of F-actin to active directional movement (radial speed) by recording centrosome movements in embryos treated with latrunculin. Consistent with previous reports (5), centrosomes separated up to a pair distance of $2-4 \mu \mathrm{m}$ (Fig. 1, $D$ and $E$ ). Based on this data set, we again calculated radial speed as a function of pair distance (Fig. $1 \mathrm{H}$ ). In contrast to wild-type embryos, radial speed was close to zero, even at small pair distances of 1 or $2 \mu \mathrm{m}$. This indicates that F-actin is involved in separation of centrosome pairs by promoting directional movement.

Our fluctuation analysis suggests that the centrosome pair separation is driven by directed forces, which are active up to a distance of $\sim 3-4 \mu \mathrm{m}$. These directed forces depend on F-actin. At larger distances or in latrunculin-injected embryos, the centrosomes move in a diffusive manner at all distances.

\section{Fluctuation parameter for fast centrosomal mobility}

In the following, we analyzed the movement of single centrosomes. Visual inspection of the centrosome movies indicate that their movement consists of a random motion on a short timescale, superimposed on large-scale drift movements together with their neighbors (Fig. $2 A$ and Movies $\mathrm{S} 1$ and S2). The latter was particularly pronounced shortly after mitosis. Two different types of network interactions may be important for active movement (Fig. 2 B). Lateral interactions exist between the centrosome pairs and their associated asters and cortical interactions of the centrosomes with the actin cortex beneath the embryonic plasma membrane.

To separate the fast and slow movements of single centrosomes, we define for each centrosome $i$ the deviation, $d_{i}(t)$, from directional movement using three time points (see Fig. $2 C$ and Fluctuations). The fluctuation parameter $D_{i}(t)=d_{i}^{2}(t) / \Delta t$ is then defined, assuming equidistant time stepping $\Delta t$ (see Eq. 2). The fluctuation parameter $D_{i}(t)$ captures the fast fluctuations around a spatially and temporally slowly varying global drift and can be calculated without knowledge of the drift and without considering the trajectories of any of the neighbors. Thus, the average $D(t)=\left(1 / N_{t}\right) \sum_{i=1}^{N_{t}} D_{i}(t)$, where $N_{t}$ is the number of centrosomes present (and visible) at time $t$, is insensitive to drift, irregular overall arrangement of centrosomes, and loss of centrosomes.

This is in contrast to a more conventional approach using the mean-square displacement (MSD). For a normal diffusive process in the absence of drift, the MSD increases linearly with time. In that case, the slope is a measure of the diffusion constant. To illustrate the different sensitivities toward drift of MSD and $D(t)$, we have performed simulations of a single particle subject to diffusion and a circular drift (Fig. 2 D). In this setting, the MSD depends unsystematically on time, whereas the fluctuation parameter remains stationary and thus is insensitive to drift.

We calculated the time series of $D(t)$ from movies of blastoderm embryos in interphase 14. (Fig. $2 E$ and Movie S2). Centrosome duplication was set to $t=0$ (Fig. $2 E$ ), i.e., each centrosome is equipped with its own clock calibrated in such a way that the time of last duplication is set to 0 . We found that the fluctuations of centrosomes are more variable before but become quite stable after centrosome duplication. As $D$ remained comparatively constant in the interphase, we averaged $D$ over the period between 60 and 260 s to obtain a time-independent parameter.

To test the sensitivity of $D$ toward experimental conditions, we first compared the two centrosome labels, Sas4 and Sas6. With both types of embryos we obtained consistent results with $D$ in the range of $4-5 \times 10^{-3} \mu \mathrm{m}^{2} / \mathrm{s}$. Our measurements are insensitive toward illumination conditions over a broad range (Fig. 2, $F$ and $G$, and Table S1). This is important as the signal-noise ratio deteriorates over time due to bleaching.

Our measurements reflect an active and energy-dependent behavior of centrosomes. Embryos depleted of ATP by injection of the poison sodium azide showed a threefold reduced $D$ (Fig. $2 F$ ). This value was in a range similar to what we measured in fixed embryos and reflects the measurement error in the positions of the centrosomes and possibly the thermal movement of the label (Fig. 2 F). Surprisingly, after these treatments, we still measured nonzero 
fluctuations. These fluctuations may be due to measurement error or thermal movement. The small difference between azide-treated and fixed embryos may be due to differences in the viscoelastic properties, which affect thermal movement. These measurements hence provide an upper limit of the measurement error, which can be inferred from Eq. 4 to be $\sigma \leq \sqrt{(\Delta t / 3) D} \approx 0.026 \mu \mathrm{m}$. For our simulations (see below), we therefore chose a value of $\sigma=0.016 \mu \mathrm{m}$ compatible with this upper limit, and which is an order of magnitude below the pixel size. Because the thermal fluctuations and measurement errors account for at most one-third of the normal fluctuations, unknown active processes appear to be responsible for a large part of the fluctuations.

On the one hand, the part of $D$ that depends on the measurement error diverges with decreasing $\Delta t$ (see Fluctuations). On the other hand, increasing $\Delta t$ leads to dependence on the drift, which can no longer be considered constant between frames. We calculated the frame-rate dependence of $D$ for one particular set of data (one wildtype embryo in interphase 14). We extended our normal imaging protocol toward a faster frame rate of $20 \mathrm{~Hz}$. The value $D$ is lowest at $\sim 1 \mathrm{~Hz}$. As expected, it increases for both lower and higher $\Delta t$. It rises to a plateau between 10 and $30 \mathrm{~s}$ (Fig. $2 H$ ), reflecting the influence of the drift. For $\Delta t<0.5 \mathrm{~s}$, we observed a sharp increase.

To confirm our interpretation that the increase of $D$ is due to either drift movement or measurement error, we computationally generated three data sets of a fluctuating particle in a velocity field, obtained as the solution of a stochastic differential equation. The first is the trajectory of the particle in a spatially and temporally constant flow field (i.e., linear drift). The second is the trajectory in a circular flow field, constant in time (i.e., circular drift). The third one is the same as the second but with additional noise (i.e., circular drift with errors, Fig. 2 I). As expected, the fluctuation parameter $D$ becomes sensitive to the drift movement at longer frame intervals, whereas at small frame intervals, the measurement error starts to dominate. The smallest influence of drift and measurement error is found between these two regimes and lies in the range of seconds for our chosen sets of parameters. It should be noted, however, that the precise location of the minimum depends on those parameters. The simulation confirms the existence of the experimentally observed minimum and indicates that the regimes dominated by drift and by measurement error are sufficiently separated. With the experimentally determined minimum at a frame interval of $\sim 1 \mathrm{~s}$, we performed all subsequent measurements at a rate of $1 \mathrm{~Hz}$.

\section{Cortical actin caps suppress centrosomal fluctuations}

The fluctuations of centrosomes may reflect passive thermal movements of the cytoplasm or active fluctuations of the dynamical cytoskeletal networks. To distinguish these options, we depolymerized F-actin by injection of latrunculin in comparison to injection of water (Fig. 3, $A$ and $B$, Movie S3, and Table S2). Surprisingly, depolymerization of F-actin resulted in an approximately twofold increase in $D$ from $5.6 \times 10^{-3}$ to $11 \times 10^{-3} \mu \mathrm{m}^{2} / \mathrm{s}$ and thus uncovered a suppressive function of $\mathrm{F}$-actin on centrosomal mobility (Fig. 3 C). This increased mobility after F-actin depolymerization is due to active, microtubule-based movement, and not unrestricted thermal/passive movement. We found that
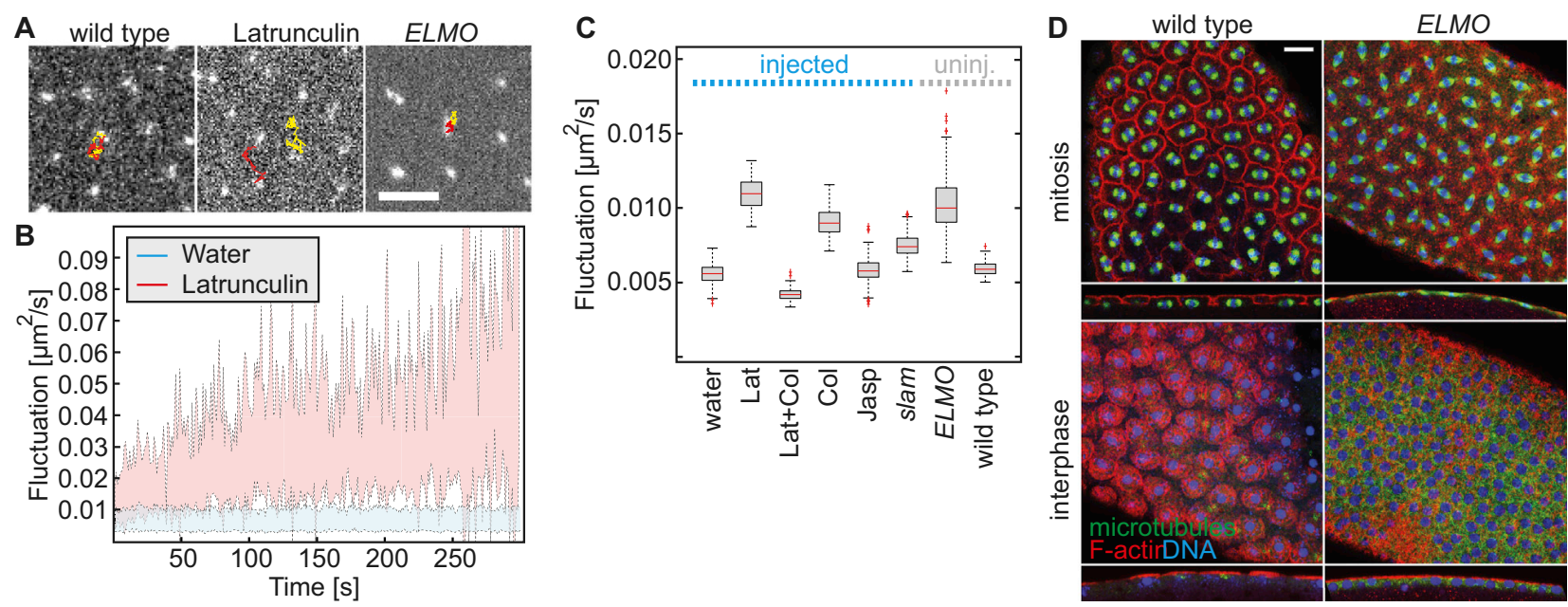

FIGURE 3 Cortical actin caps suppress fluctuations. (A) Images from time-lapse recordings of wild-type, latrunculin-injected, and ELMO embryos expressing Sas6-GFP. Centrosome trajectories (120 s) are marked (red and yellow). Scale bar $=5 \mu$ m. $(B)$ Time courses of fluctuation (range of standard deviation) in embryos injected with water or latrunculin in mitosis interphase 13. (C) Time-averaged fluctuation in embryos with indicated treatment (injected) or genotype (not injected). Latrunculin (Lat) + Colcemid (Col) coinjection; injected with Jasplakinolide (Jasp); and depleted by slam RNA (slam). Statistical significance of all pairs is listed in Table S3. (D) Images of wild-type and ELMO embryos stained for F-actin (red), microtubules ( green), and DNA (blue). Small images show sagittal section of the same embryo. Scale bar $=10 \mu \mathrm{m}$. 
coinjection of latrunculin and colcemid lead to a loss of the increased fluctuation to $D=4.2 \times 10^{-3} \mu \mathrm{m}^{2} / \mathrm{s}$, in the range of the value measured in water-injected embryos (Fig. $3 C$ and see Movie S4). Depolymerization of microtubules by injection of colcemid also lead to increased fluctuations with $D=9.1 \times 10^{-3} \mu \mathrm{m}^{2} / \mathrm{s}$ (Fig. $3 C$ ). Thus, microtubules also stabilize centrosome movement and may have a dual function, as they are also required for stronger fluctuations in the absence of F-actin. Conversely to depolymerization, stabilization of F-actin by Jasplakinolide (Jasp) did not affect fluctuations, indicating that the assembly-disassembly cycle of actin filaments is not required for suppression of fluctuations by F-actin (Fig. $3 C$ ).

During interphase, F-actin accumulates in actin caps above centrosomes. To assess whether the caplike distribution of F-actin is important for centrosome mobility, we employed ced-12/ELMO mutant embryos that lack actin caps and display uniform cortical actin (Fig. 3 D). The ced-12/ ELMO embryos pass through syncytial cell cycles with a timing comparable to wild-type embryos (data not shown). However, metaphase furrows did not form (Fig. 3 D), chromosome segregation defects were frequently observed, and cellularization failed (data not shown). We introduced the centrosome marker Sas6-GFP into these embryos and recorded centrosome movement. We measured an average of $D=10.3 \times 10^{-3} \mu \mathrm{m}^{2} / \mathrm{s}$, which lies in the range of $D$ that we measured in latrunculin-injected embryos (Fig. 3 $C$ and see Movie S5). These data suggest that the organization of F-actin into caps is important for the suppression of centrosome fluctuations. Unstructured cortical F-actin as present in ced-12/ELMO mutants does not suffice for stabilization of centrosomes.

During interphase 14, development switches from syncytial to cellular development. The plasma membrane invaginates between adjacent nuclei to enclose nuclei into individual cells. Furrow invagination is associated with reor- ganization of F-actin. The cortical actin caps dissolve, while the furrow canal, the tip of the invagination membrane, acquires an actin coat. We tested whether centrosomal fluctuations are affected by this reorganization in cellular and cytoskeletal structure by analyzing slam mutant embryos. Slam is required for invagination of furrows. We depleted slam by injection of slam dsRNA into embryos expressing the centrosomal marker Sas6-GFP. Induction of the mutant phenotype was verified by the absence of furrow invagination. Quantification of centrosome fluctuations in these embryos revealed a slight increase of fluctuations with $D=7.5 \times 10^{-3} \mu \mathrm{m}^{2} / \mathrm{s}$ (Fig. $3 C$ ). These data suggest that the invaginating furrows may only weakly suppress fluctuations.

\section{Role of molecular motors Myosin-II and Kinesin-1}

Active fluctuations of the centrosomes may be promoted by molecular motors. Potential candidates are myosin motor proteins for the actin cytoskeleton. To assess the role of nonmuscle Myosin-II, we interfered with its function by inhibiting Rho kinase, which is a well-characterized activator of Myosin-II by injection of the chemical inhibitor Y-27632 (5). Its functionality was verified by the loss of localized fluorescence of Myo-II-GFP (data not shown). We recorded movies of Y-27632-injected embryos expressing Sas6-GFP. The calculated centrosomal fluctuations were slightly increased to $D=8.1 \times 10^{-3} \mu \mathrm{m}^{2} / \mathrm{s}$, which corresponds to an $\sim 50 \%$ increase as compared to water-injected embryos (Fig. $4 C$ ). Thus, these data suggest that Myosin-II, as much as it is affected by the inhibition of Rho kinase, does not promote centrosomal fluctuations but rather contributes to suppression of fluctuations by F-actin.

Concerning molecular motors of the microtubules, a prime candidate is Kinesin-1. Kinesin-1 is not required for mitosis, and mutant embryos could be obtained (14).
A

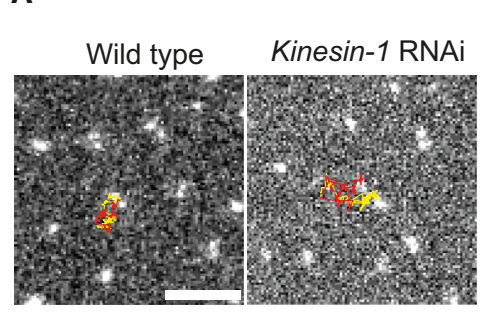

B

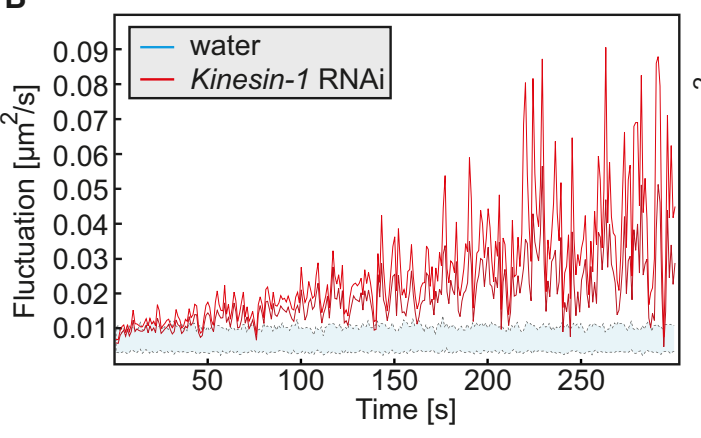

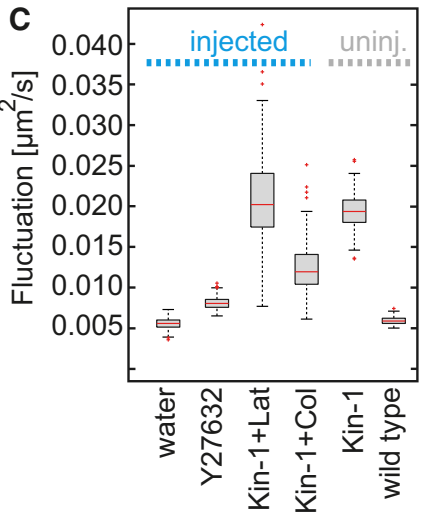

FIGURE 4 Kinesin-1 suppresses centrosome fluctuations. (A) Images from time-lapse recordings of wild-type and Kinesin-1 mutant embryos expressing centrosomal GFP markers. Centrosome trajectories (120 s) are marked (red and yellow). Scale bar $=5 \mu \mathrm{m}$. $(B)$ Time courses of fluctuation (range of standard deviation) in wild-type and Kinesin-1 mutant embryos. (C) Time-averaged fluctuation in embryos with indicated treatment (injected) or genotype (not

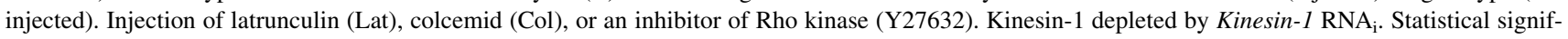
icance of all pairs is listed in Table S3. 
Kinesin-1 mutant embryos developed normally until interphase 14 but did not cellularize (Fig. S1 and Movies S6, S7, S8, and S9). Expression of Kinesin-1 dsRNA in the germline leads to a similar phenotype. Furthermore, ens mutant embryos showed a similar phenotype (Fig. S1). Ens functions together with Kinesin-1 and is involved in loading of Kinesin-1 onto microtubules (14). In the following, we employed Kinesin-1 $\mathrm{RNA}_{\mathrm{i}}$ for the sake of experimental simplicity. We first stained wild-type and $K i$ nesin-1 mutant embryos for F-actin and microtubules (data in Fig. S2, $A$ and $B$ ). We did not observe any difference in microtubule asters during syncytial cycles and nuclear baskets in interphase 14. F-actin distribution, i.e., actin caps in interphase and metaphase furrows during mitosis, was also similar in wild-type and Kinesin-1 mutants. We recorded centrosomal fluctuations in embryos expressing Kinesin-1 dsRNA and Sas4-GFP (Fig. 4 A). Surprisingly, Kinesin-1 was required for suppression of centrosome fluctuation rather than promotion, as one might expect for a motor (Fig. 4 B). The fluctuation parameter $D$ increased by more than threefold as compared to wild-type embryos to $D=19.4 \times 10^{-3} \mu \mathrm{m}^{2} / \mathrm{s}$ (Fig. $4 C$ and Table S2). These data suggest that the microtubule motor Kinesin-1 is involved in suppression of centrosome mobility. The increased fluctuation in embryos depleted for Kinesin-1 was not further increased by injection of latrunculin (Fig. 4 C). As actin depolymerization in Kinesin-1 mutants did not increase the fluctuations, cortical actin and Kinesin1 may act in the same pathway. Conceivably, Kinesin-1 may establish the linkage between the microtubule network and the cortical actin caps.

In contrast, the increased fluctuations in Kinesin-1depleted embryos depended partly on microtubules. Depolymerization of microtubules by injection of colcemid reduced fluctuations to $D=12.7 \times 10^{-3} \mu \mathrm{m}^{2} / \mathrm{s}$. This indicates that the fluctuation movements uncovered after depletion of Kinesin-1 are mediated by microtubules and conceivably by motors other than Kinesin-1. This observation disfavors an alternative model for Kinesin-1 function, namely in lateral interactions: in such a model, Kinesin-1 cross-links overlapping microtubule asters and stabilizes the microtubule network. Correspondingly, loss of Kinesin-1 would lead to higher mobility of the loosened centrosomes. An additional depolymerization of microtubules on top of Kinesin-1 depletion would not change the mobility.

A prediction of a function of Kinesin-1 in cortical interactions of the microtubule network is a localization of Kine$\sin -1$ at the cortex. Hence, we analyzed the distribution of Kinesin-1 protein. We imaged Kinesin-1 in transgenic flies expressing Kinesin-1-GFP (14). Movies of such embryos showed that Kinesin-1-GFP accumulated at cortical actin caps in interphase and spindles in mitosis (Fig. 5, $A$ and $B)$. Such a cortical localization of Kinesin-1 is consistent with a function of Kinesin-1 in linking microtubule asters to the cortex. Astral microtubules are oriented with their $(+)$-ends toward the cortical actin caps, where the $(+)$-end directed motor Kinesin-1 accumulates and may serve as a
A

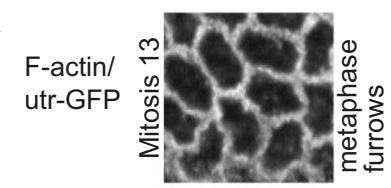

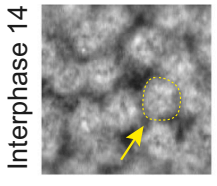

C

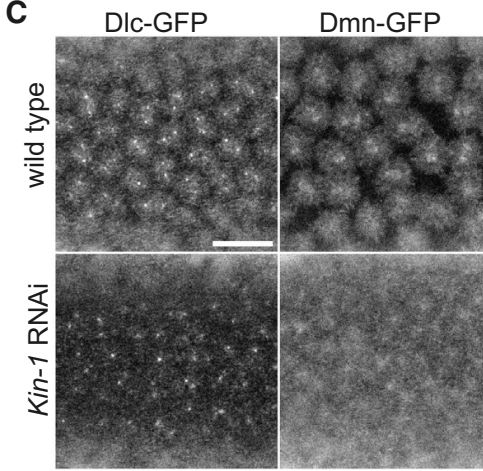

D

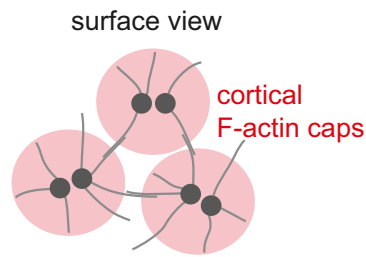

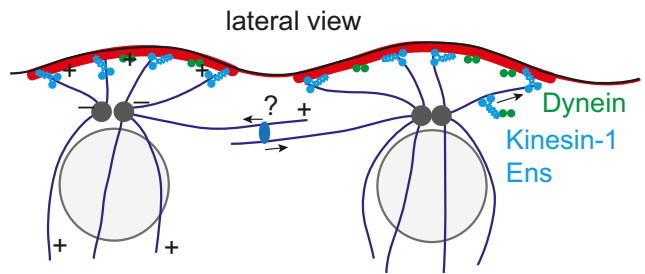

FIGURE 5 Kinesin-1 is required for cortical Dynein. Images from time-lapse recordings of embryos expressing $(A)$ F-actin marker utr-GFP (utrophin); $(B)$ Kinesin-1-GFP; and $(B$ and $C$ ) Dlc-GFP (Dynein light chain) and Dmn-GFP (Dynamitin) between interphases 13 and 14. (A) Scheme indicates structure of actin caps (arrow, dashed circle) with centrosome pairs (black dots) in interphase. $(C)$ Images of wild-type and Kinesin-1 mutant embryos in interphase 14. (D) Model for the interaction of centrosomes with the cortex and suppression of centrosome fluctuations by cortical F-actin and Kinesin-1. Scale bars $=10 \mu \mathrm{m}$. 
linker between the $(+)$-ends of the microtubules and F-actin (Fig. 5 D).

The function and subcellular dynamics of Kinesin-1 has not yet been investigated in early Drosophila embryos, to our knowledge. Previously, a function of Kinesin-1 in germ plasm formation during oogenesis and subsequent anterior-posterior pattern formation was described in Brendza et al. (10) and Januschke et al. (11). In the developing oocyte, Kinesin-1 transports the (-)-end directed motor Dynein to the posterior cortex, where Kinesin-1 and Dynein colocalize and are assumed to anchor germ plasm and posterior determinants. For the early embryo, it has been reported that Dynein accumulates at the actin caps (43), although its functions have remained unclear. It is assumed that Dynein contributes to formation and function of the mitotic spindle. The mechanism for its localization at the cortical actin caps in interphase has remained unknown, however. We tested whether the presence of Dynein at the cortex depends on Kinesin-1 in the blastoderm embryo. We confirmed the cortical localization of Dynein with movies of transgenic embryos expressing two components of the Dynein complex, either Dlc-GFP (Dynein light chain) or Dmn-GFP (Dynamitin) (15). Cortical localization of the Dynein complex depended on Kinesin-1 (Fig. 5 C). Embryos, in which Kinesin-1 was depleted by $\mathrm{RNA}_{\mathrm{i}}$, lost the clear cortical caplike localization of both Dlc-GFP and Dynamitin-GFP, whereas the centrosomal signal remained clear (Fig. 5 C). These data suggest that cortical localization of Dynein depends on Kinesin-1, whereas other aspects of Dynein localization, such as centrosomal localization, are independent of Kinesin-1.

\section{DISCUSSION}

Our data are consistent with a model that Kinesin-1 mediates cortical anchoring of microtubule asters (Fig. $4 \mathrm{D}$ ). This extends a previous model that Dynein links mitotic spindles to the cortex in syncytial embryos and contribute to lengthening of the spindle in anaphase (43). The previously proposed model is focused on mitosis and does not provide an explanation for a potential function and for the localization of the (-)-end directed motor Dynein in the region of the $(+)$-ends of microtubules asters in interphase. We propose that interactions between the microtubule network and the actin cortex are comparable in interphase and during mitosis, both involving cortical microtubule motors as a link between microtubules and cortical actin. Kinesin-1 may directly interact with components of the actin cap and anchor the microtubule (+)-ends to the cortex. In this model the dependence of cortical Dynein localization on Kinesin-1 would not be relevant for microtubule anchoring. Alternatively, Kinesin-1 may act indirectly, in that it transports Dynein to the (+)-ends with the help of Ensconsin. After reaching the cortex, Dynein then serves as an anchor for microtubules. As both models are not mutually exclusive, Kinesin may first transport Dynein to the cortex and then together with Dynein provide a cortical link.

Such a function of Kinesin-1 would not have been discovered by analysis of static images or slow movies. In contrast to more severe interference, such as depolymerization of F-actin, which leads to visible morphological defects, the cytoskeletal organization, as seen in fixed embryos, is not obviously disturbed. The differences in cytoskeletal mobility between wild-type and mutant embryos, however, are revealed by the dynamics of centrosomes.

The dynamics of the cytoskeleton in the complex embryo is determined by multiple parameters acting on many length and timescales. We introduced a fluctuation parameter, which allowed us to separate large-scale and slow (range of minutes) drift movement from fast stochastic motion in a timescale relevant for cytoskeletal dynamics. If the density was high enough, the drift could be estimated by averaging over the movement of several neighbors. In mutant or injected embryos with irregular morphology, neighborhood relations are difficult to establish, however.

We also addressed the question of whether the structure of cortical actin is important for stabilization of centrosomal mobility by comparing wild-type with caplike organization and mutant embryos with uniform F-actin distribution (ced-12/ELMO mutants). We provide evidence that the caplike structuring of cortical actin clearly contributes to stabilization of centrosomal mobility, as fluctuations were similar in latrunculin-injected and ced-12/ELMO embryos. Interesting in this respect is the fact that the F-actin caps are induced by the underlying centrosomes (4) and that caps feed-back to influence centrosome mobility, effectively constituting a mutual functional relationship.

So far, we have identified only components that stabilize centrosome fluctuations. Indirect inhibition of Myosin-II and depletion of Kinesin-1 both lead to increased fluctuations. For a motor driving centrosome mobility, we would expect a reduced fluctuation parameter $D$, if this motor is inhibited or genetically depleted. Based on our data, we expect that active components exist and that they act on both F-actin and microtubules. Depolymerization of both F-actin and microtubules lead to a reduced fluctuation parameter $D$, whereas depolymerization of either F-actin or microtubules lead to an increased $D$. This indicates that a microtubule-dependent motor drives the higher fluctuation in the case of F-actin depolymerization, and an F-actin-dependent motor, in the case of microtubule depolymerization. Furthermore, depolymerization of microtubules in Kinesin-1-depleted embryos lead to reduced fluctuations. This indicates that the increased centrosomal mobility in such embryos depends on intact microtubules. The high fluctuations may be due to a microtubule motor.

A promising candidate for the microtubule network is Kinesin-5, which is a four-headed molecular motor 
involved in antiparallel sliding of microtubules and in spindle elongation during mitosis (44-46). During mitosis, Kinesin-5 is involved in antiparallel sliding of microtubules. This activity is assumed to contribute to elongation of the spindle in anaphase. Kinesin-5 may have a similar function during interphase in syncytial embryos. Using a GFP-tagged Kinesin-5 we have observed dynamical localization of Kinesin- 5 on the microtubule asters (unpublished data). Kinesin-5 may locate at positions, where microtubules from adjacent microtubule asters arrange in an antiparallel manner and may contribute to repulsion of adjacent asters.

In this study, we employed an approach to analyze the dynamics of centrosomes in that the individual paths including fluctuations were treated as a source of information instead of being simply averaged. The fluctuations are caused by thermal forces and importantly, active nonequilibrium forces of the actin and microtubule cytoskeleton. The contribution by active forces was uncovered by depolymerization of the cytoskeleton. Ideally, we would like to record centrosome dynamics in a frequency space of up to $100 \mathrm{~Hz}$, providing a more comprehensive description of the material properties of the embryo. Together with the yet-to-be-determined viscoelastic properties of the embryos, this would allow quantification of the active force contribution by the cytoskeleton. However, we are limited to frame rates in the second range with GFP-based probes. Using innovative fluorescent labels such as single-walled carbon nanotubes targeted to centrosomes may provide a means to overcome the limits of GFP labels (8).

\section{SUPPORTING MATERIAL}

Two figures, three tables, and nine movies are available at http://www. biophysj.org/biophysj/supplemental/S0006-3495(15)00779-1.

\section{AUTHOR CONTRIBUTIONS}

J.G. designed the experimental approach; A.Z. and T.A. conceived the method for quantitative data analysis; M.G. and F.W. performed the experiments; L.K. performed the data analysis; and Z.L. conducted the experiments concerning ELMO.

\section{ACKNOWLEDGMENTS}

We thank M. Baylies, M. Bettencourt-Dias, T. Hays, T. Lecuit, P. Rorth, J. Raff, F. Schnorrer, H. Sung, and S. Tsukita, and the Developmental Hybridoma Band at the University of Iowa, the Bloomington Drosophila Stock Center, the Genomic Resource Center at Indiana University, and the Transgenic RNA $_{\mathrm{i}}$ Project at Harvard Medical School (funded by grant No. RO1GM084947 from the National Institutes of Health/National Institute of General Medical Sciences) for discussions, materials, or fly stocks. We thank T. Kanesaki for initial work.

This work was supported in part by the German Research Council (under Sonderforschungsbereich (SFB) grant No. 937/TP A10).

\section{REFERENCES}

1. Foe, V. E., G. M. Odell, and B. A. Edgar. 1993. Mitosis and morphogenesis in the Drosophila embryo: point and counterpoint. In The Development of Drosophila melanogaster. M. Bate and A. Martinez Arias, editors. Cold Spring Harbor Laboratory Press, Cold Spring Harbor, NY.

2. Schejter, E. D., and E. Wieschaus. 1993. Functional elements of the cytoskeleton in the early Drosophila embryo. Annu. Rev. Cell Biol. 9:67-99.

3. Kanesaki, T., C. M. Edwards, ..., J. Grosshans. 2011. Dynamic ordering of nuclei in syncytial embryos: a quantitative analysis of the role of cytoskeletal networks. Integr. Biol. (Camb). 3:1112-1119.

4. Raff, J. W., and D. M. Glover. 1989. Centrosomes, and not nuclei, initiate pole cell formation in Drosophila embryos. Cell. 57:611-619.

5. Cao, J., J. Crest, ..., W. Sullivan. 2010. Cortical actin dynamics facilitate early-stage centrosome separation. Curr. Biol. 20:770-776.

6. Robinson, J. T., E. J. Wojcik, ..., T. S. Hays. 1999. Cytoplasmic dynein is required for the nuclear attachment and migration of centrosomes during mitosis in Drosophila. J. Cell Biol. 146:597-608.

7. Sommi, P., D. Cheerambathur, ..., A. Mogilner. 2011. Actomyosindependent cortical dynamics contributes to the prophase force-balance in the early Drosophila embryo. PLoS One. 6:e18366.

8. Fakhri, N., A. D. Wessel, ..., C. F. Schmidt. 2014. High-resolution mapping of intracellular fluctuations using carbon nanotubes. Science. 344:1031-1035.

9. Yang, J. T., W. M. Saxton, and L. S. Goldstein. 1988. Isolation and characterization of the gene encoding the heavy chain of Drosophila kinesin. Proc. Natl. Acad. Sci. USA. 85:1864-1868.

10. Brendza, R. P., L. R. Serbus, ..., J. B. Duffy. 2002. Posterior localization of dynein and dorsal-ventral axis formation depends on kinesin in Drosophila oocytes. Curr. Biol. 12:1541-1545.

11. Januschke, J., L. Gervais, ..., A. Guichet. 2002. Polar transport in the Drosophila oocyte requires Dynein and Kinesin-I cooperation. Curr. Biol. 12:1971-1981.

12. Metzger, T., V. Gache, ..., M. K. Baylies. 2012. MAP and kinesindependent nuclear positioning is required for skeletal muscle function. Nature. 484:120-124.

13. Pilling, A. D., D. Horiuchi, ..., W. M. Saxton. 2006. Kinesin-1 and Dynein are the primary motors for fast transport of mitochondria in Drosophila motor axons. Mol. Biol. Cell. 17:2057-2068.

14. Sung, H. H., I. A. Telley, ..., P. Rørth. 2008. Drosophila Ensconsin promotes productive recruitment of Kinesin-1 to microtubules. Dev. Cell. $15: 866-876$.

15. Wojcik, E., R. Basto, ..., T. Hays. 2001. Kinetochore dynein: its dynamics and role in the transport of the Rough deal checkpoint protein. Nat. Cell Biol. 3:1001-1007.

16. Peel, N., N. R. Stevens, ..., J. W. Raff. 2007. Overexpressing centriolereplication proteins in vivo induces centriole overduplication and de novo formation. Curr. Biol. 17:834-843.

17. Luschnig, S., B. Moussian, ..., C. Nüsslein-Volhard. 2004. An F1 genetic screen for maternal-effect mutations affecting embryonic pattern formation in Drosophila melanogaster. Genetics. 167:325-342.

18. Mazzalupo, S., and L. Cooley. 2006. Illuminating the role of caspases during Drosophila oogenesis. Cell Death Differ. 13:1950-1959.

19. Rauzi, M., P.-F. Lenne, and T. Lecuit. 2010. Planar polarized actomyosin contractile flows control epithelial junction remodelling. Nature. 468:1110-1114.

20. Oda, H., and S. Tsukita. 2001. Real-time imaging of cell-cell adherens junctions reveals that Drosophila mesoderm invagination begins with two phases of apical constriction of cells. J. Cell Sci. 114:493-501.

21. Schnorrer, F., K. Bohmann, and C. Nüsslein-Volhard. 2000. The molecular motor dynein is involved in targeting swallow and bicoid RNA to the anterior pole of Drosophila oocytes. Nat. Cell Biol. 2:185-190. 
22. Wenzl, C., S. Yan, ..., J. Grosshans. 2010. Localization of RhoGEF2 during Drosophila cellularization is developmentally controlled by Slam. Mech. Dev. 127:371-384.

23. Hafi, N., M. Grunwald, ..., P. J. Walla. 2014. Fluorescence nanoscopy by polarization modulation and polarization angle narrowing. Nat Methods. 11. http://dx.doi.org/10.1038/nmeth.2919.

24. Boufounos, P., M. F. Duarte, and R. G. Baraniuk. 2007. Sparse signal reconstruction from noisy compressive measurements using cross validation. In Proceedings of the IEEE Workshop on Statistical Signal Processing (SSP). Institute of Electrical and Electronics Engineers, Piscataway, NJ, pp. 299-303.

25. Frick, K., P. Marnitz, and A. Munk. 2012. Statistical multiresolution estimation for variational imaging: with an application in Poisson biophotonics. J. Math. Imaging Vis. 46:370-387.

26. Babcock, H. P., J. R. Moffitt, ..., X. Zhuang. 2013. Fast compressed sensing analysis for super-resolution imaging using L1-homotopy. Opt. Express. 21:28583-28596.

27. Mukamel, E. A., H. Babcock, and X. Zhuang. 2012. Statistical deconvolution for superresolution fluorescence microscopy. Biophys. J. 102: 2391-2400.

28. Zhu, L., W. Zhang, ..., B. Huang. 2012. Faster STORM using compressed sensing. Nat. Methods. 9:721-723.

29. Fortin, M., and R. Glowinski. 1983. Chapter III. Decomposition-coordination methods using an augmented Lagrangian. In Augmented Lagrangian Methods: Applications to the Numerical Solution of Boundary-Value Problems. Elsevier, New York, pp. 97-146.

30. Gal, N., D. Lechtman-Goldstein, and D. Weihs. 2013. Particle tracking in living cells: a review of the mean square displacement method and beyond. Rheol. Acta. 52:425-443.

31. Munk, A., N. Bissantz, ..., G. Freitag. 2005. On difference-based variance estimation in nonparametric regression when the covariate is high dimensional. J. R. Stat. Soc. Series B Stat. Methodol. 67:19-41.

32. Lau, A. W. C., B. D. Hoffman, ..., T. C. Lubensky. 2003. Microrheology, stress fluctuations, and active behavior of living cells. Phys. Rev Lett. 91:198101.

33. Wessel, A. D., M. Gumalla, ..., C. F. Schmidt. 2015. The mechanical properties of early Drosophila embryos measured by high-speed video microrheology. Biophys. J. 108:1899-1907.
34. Savin, T., and P. S. Doyle. 2005. Role of a finite exposure time on measuring an elastic modulus using microrheology. Phys. Rev. E Stat. Nonlin. Soft Matter Phys. 71:041106.

35. Savin, T., and P. S. Doyle. 2005. Static and dynamic errors in particle tracking microrheology. Biophys. J. 88:623-638.

36. Sainis, S. K., V. Germain, and E. R. Dufresne. 2007. Statistics of particle trajectories at short time intervals reveal fN-scale colloidal forces. Phys. Rev. Lett. 99:018303.

37. Levine, A J and T C Lubensky, 2001. Response function of a sphere in a viscoelastic two-fluid medium. Phys. Rev. E Stat. Nonlin. Soft Matter Phys. 63:041510.

38. Lim, H. H., T. Zhang, and U. Surana. 2009. Regulation of centrosome separation in yeast and vertebrates: common threads. Trends Cell Biol. $19: 325-333$.

39. Levine, A. J., and T. C. Lubensky. 2000. One- and two-particle microrheology. Phys. Rev. Lett. 85:1774-1777.

40. MacKintosh, F. C., and C. F. Schmidt. 1999. Microrheology. Curr. Op. Coll. Inter. Sci. 4:300-307.

41. Weihs, D., T. G. Mason, and M. A. Teitell. 2006. Bio-microrheology: a frontier in microrheology. Biophys. J. 91:4296-4305.

42. Wirtz, D. 2009. Particle-tracking microrheology of living cells: principles and applications. Annu. Rev. Biophys. 38:301-326.

43. Cytrynbaum, E. N., P. Sommi, ..., A. Mogilner. 2005. Early spindle assembly in Drosophila embryos: role of a force balance involving cytoskeletal dynamics and nuclear mechanics. Mol. Biol. Cell. 16: 4967-4981.

44. Brust-Mascher, I., P. Sommi, ..., J. M. Scholey. 2009. Kinesin5-dependent poleward flux and spindle length control in Drosophila embryo mitosis. Mol. Biol. Cell. 20:1749-1762.

45. Heck, M. M., A. Pereira, .... L. S. Goldstein. 1993. The kinesin-like protein KLP61F is essential for mitosis in Drosophila. J. Cell Biol. 123:665-679.

46. Sharp, D. J., K. R. Yu, ..., J. M. Scholey. 1999. Antagonistic microtubule-sliding motors position mitotic centrosomes in Drosophila early embryos. Nat. Cell Biol. 1:51-54. 



\section{Discussion}

The aim of this project was to understand the mechanisms of the cytoskeletal dynamics. The cytoskeleton is a dynamic network, which is formed by actin and microtubules. The early syncytial embryo of Drosophila is a system well suited to investigate cytoskeletal networks and functional interactions between microtubule asters and the actin cortex. Since no cell membranes separate the cellular units, the cytoskeleton forms a potentially large network, which extends over the whole embryo $[14,36]$. Due to the facts that microtubule asters overlap with each other and emanate from centrosomes as well as that the nuclei are anchored to the cortex by and their movement depends on centrosomes, we used centrosomes and their movement as a proxy for network dynamics and nuclear movement. Embryos expressing GFP-tagged centriolar components were recorded and pair separation and individual movement of centrosomes were analysed to reveal mechanisms, which are responsible for network dynamics and stabilise the regular organisation of the nuclear array.

First, we observed the natural, undisturbed system to identify the properties of the centrosomal / nuclear network. An in vivo assay of microtubule network dynamics was established, a quantitative assay to record and analyse centrosome movement. At the end of mitosis, centrosomes moved from the spindle poles to an apical position between the nuclei and the cortex, where they duplicated. In interphase the centrosome pair separated and moved to opposing equatorial positions of the nuclei before nuclear envelope breakdown. We were mainly interested in the interphase movement, which occurred prior to equatorial movement. Thereby we found out that the average pair distance increased first slowly and then rapidly. The latter separation corresponds to the equatorial movement.

After observing the natural, undisturbed system, we performed experiments using mutant embryos and drug injections to reveal the function of specific components and regulators of the cytoskeleton. Thereby we observed that separation of the centrosome pairs proceeded in a diffusive manner after depolymerisation of F-actin by latrunculin A injection. We concluded F-actin as being required for initial centrosome pair separation. This was confirmed by results obtained by Cao and co-workers [9]. They could show that centrosome separation prior to nuclear envelope breakdown requires dynamic actin rearrangements at the actin cap. Inhibition of this remodelling, either by drug injection of by mutants with reduced or absent actin caps, resulted in a failure of centrosome separation and occurrence of multipolar spindles during mitosis. They mentioned that actin depolymerisation only affects the separation, but not splitting of centrosomes. Our 
results confirmed this observation. Both centrosomes were clearly visible, but unable to properly separate from each other. The authors assumed that the Apc2-Armadillo complex could link cap expansion to centrosome separation. To test, if this complex is responsible for the proper F-actin mediated separation of centrosomes, embryos injected with a specific and highly concentrated antibody, which blocks the function of Apc2 or Armadillo in the embryo could be recorded and their centrosome movement extracted.

Visual inspection of the centrosome movement in living Drosophila embryos indicated that the overall movement is a combination of two separate movements. A random movement on a short time-scale is superimposed on large-scale drift movements together with their neighbours. The latter is particularly pronounced shortly after mitosis. Injection of sodium azide, which depletes the embryo from ATP reduced the centrosome fluctuations, the random movement on a short time-scale, to a value similar to fixed embryos. This indicated that centrosome fluctuations are active movements. This was confirmed by analysis of the fluctuations in embryos with abolished F-actin structure. Depolymerisation of $\mathrm{F}$-actin by latrunculin $\mathrm{A}$ injection increased centrosome fluctuation by a factor of two, indicating that F-actin suppresses centrosomal fluctuations. Further experiments revealed, that this is mediated by actin caps. Furthermore we could show that these increased fluctuations are dependent on microtubule structures, since depletion of both F-actin and microtubules led to a loss of the increased mobility. However, single depletion of microtubules increased mobility, indicating that microtubules could have a dual function in promoting and also stabilising centrosome movement. Surprisingly stabilisation of F-actin by jasplakinolide injection did not change the mobility of centrosomes, which allow the conclusion that F-actin turnover is not required to suppress fluctuations. Considering that F-actin caps are induced by the underlying centrosomes [34] and that the caps feed-back to influence centrosome mobility, a functional relationship influencing and controlling each other is constituted (Figure 63).

Our result that microtubules could both promote and suppress the active centrosome mobility indicates that motor proteins could be involved, which modulate microtubule function. So far, there are three major candidate motor proteins: Kinesin-1 and Kinesin5 as microtubule motor proteins, and Myosin-II as an actin cytoskeleton motor protein. We identified non-muscle Myosin-II as a suppressor of fluctuations induced by F-actin. Injection of Y-27632, a chemical inhibitor of the Myosin-activating Rho kinase, slightly increased fluctuations of centrosomes. This mild increase in comparison to the strong fluctuation increase obtained by F-actin depolymerisation indicated the participation of further motor proteins.

Kinesin- 1 is a $(+)$-end directed microtubule motor protein, which is located at the actin cortex in syncytial Drosophila embryos. Therefore a function in cortical interactions of the microtubule network seems to be likely. Astral microtubules are anchored to centrosomes with their (-)-ends. Their $(+)$-ends are oriented towards the cortical actin caps, where the $(+)$-end directed motor protein Kinesin-1 accumulates and may serve as 
a linker to F-actin. We revealed that Kinesin-1 is required for suppression of centrosomal fluctuations, because centrosome mobility was increased by a factor of three after application of Kinesin-1 RNAi. Since the fluctuation parameter did not change after co-depletion of polymerised F-actin, it can be assumed that Kinesin-1 and cortical actin may act in the same pathway. Thereby, Kinesin-1 may establish a linkage between the microtubule network and the cortical actin caps (Figure 63). It can be excluded that Kinesin-1 and microtubule function in suppressing centrosome mobility occurs in the same pathway, because depolymerisation of microtubules reduced the mobility in kinesin-1 depleted embryos. This indicates the participation of further motor proteins other than Kinesin-1 and Myosin-II.

It is known that Kinesin-1 transports the (-)-end directed motor protein Dynein to the posterior cortex of syncytial Drosophila embryo, where both colocalise and are assumed to anchor posterior determinants and germ plasm $[5,19]$. In actin caps an interaction of Kinesin- 1 and Dynein is also likely, because Dynein accumulates similarly to Kinesin-1 at the actin cortex [12]. Furthermore it is assumed to be involved in mitotic spindle formation and function. We confirmed this interaction, since the cortical localisation of Dynein was lost in kinesin-1 depleted embryos. Other aspects of Dynein localisation, such as centrosomal localisation, were independent of Kinesin-1. This extends a model, in which Dynein links mitotic spindles to the cortex in syncytial embryos and contribute to lengthening of the spindle in anaphase [12]. This model is focused on mitosis and do not provide explanations for potential functions in interphase. We could extend this model by Kinesin-1 mediating the cortical anchoring of Dynein and therefore microtubules (Figure 63). This would explain the localisation of the (-)-end directed motor protein Dynein in a region of $(+)$-ends of microtubules. The expanded model proposes that the interaction between the actin cortex and the microtubule network is comparable both in interphase and mitosis, with both involving cortical microtubule motors as links between them. Currently it can not be excluded, if Kinesin-1 anchors microtubules indirectly via localisation of Dynein or if it first transports Dynein to the cortex and then provides a cortical link together with Dynein. To distinguish both possibilities, a kinesin-1 mutant could be generated and introduced into kinesin-1 depleted embryos, which lacks the catalytic activity, but is fully able to localise and transport Dynein.

Kinesin-1 mediated anchoring of microtubule asters to the cortex could work in a similar way as revealed for cytoplasmic streaming in Drosophila oocytes [29]. In oocytes, it is important to mix organelles and cytoplasm from nurse cells with ooplasm. This is achieved by cytoplasmic streaming. Microtubules form a disordered meshwork beneath the cortex, which allows a slow cytoplasmic flow. Kinesin-1 induces the formation of a subcortical layer of parallely arranged microtubules, which supports fast and long-ranged flows. In syncytial Drosophila embryos Kinesin-1 could also be involved in arranging the microtubules to transport Dynein to its target sites for anchoring the microtubule asters and centrosomes. This goes in line with results obtained from Melkow and co-workers [27]. Bristle cells are among the largest cells in the peripheral nervous system and 
their morphology depends on Kinesin heavy chain function. It has been proposed that in kinesin heavy chain depleted flies the transport of essential membrane precursors is affected [4]. Melkow et al. revealed that the Kinesin-1 complex acts via reorganisation of microtubules into uniformly oriented microtubule bundles to allow the highly biased axial growth, which is necessary to obtain the proper bristle structure and function. Furthermore, recently it has been shown that Dynein is involved in establishing proper axon development [10]. During axon outgrowth, the originally mixed orientation of microtubules switches to a uniform polarity with $(+)$-end out, which is a Dyneindependent process. Since bristle cells are cells of the peripheral nervous system also establishing a switch in microtubule organisation, a similar function of Dynein could be estimated, which would indicate an interaction of Kinesin-1 and Dynein. The interdependence of Kinesin-1 and Dynein was further confirmed by a recent genome-wide RNAi screen for the formation of microtubule bundles and regulation of lysosome motility [20]. This screen was performed in actin depleted Drosophila S2 cells, which lack intermediate filaments CentroGoldstein2000, leaving microtubules as only organelle for organelle transport.

Recent data from Arora and colleagues suggests that not always the absolute amount of a motor protein is decisive for a specific function, but rather the ratio between levels of several proteins to each other CentroArora2016. They analysed lipid droplet transport in early Drosophila embryos and could show that the ratio between Kinesin-1 and Halo protein level is crucial for the net transport of this bidirectional cargo. It would be interesting to test, if this is also true for the Kinesin-1/Dynein interaction in syncytial embryos.

So far, only components were identified, which stabilise centrosome fluctuations. Based on the data, we expect that fluctuation promoting components exist and that they act both on F-actin and microtubules. Evidence for this is the observation that depolymerisation of both F-actin and microtubules or Kinesin-1 and microtubules led to reduced fluctuation, whereas single depolymerisation increased them. A promising candidate for a fluctuation promoting protein is Kinesin-5, which is a four-headed microtubule motor protein involved in antiparallel sliding of microtubules and in spindle elongation during mitosis $[6,17,38]$. If the mitotic function of Kinesin-5 is affected, abnormal monoastral spindles are often formed, which indicates a contribution in maintaining of spindle bipolarity and separation by sliding apart microtubules (see [6] and references therein). Kinesin-5 may have a similar function during interphase. Observation of GFP-tagged Kinesin-5 showed a dynamical localisation on microtubules asters (unpublished data). We hypothesize that Kinesin-5 could locate at positions, where microtubules from adjacent microtubule asters overlap and arrange in an antiparallel manner to contribute to repulsion of adjacent asters (Figure 63). Addressing Kinesin-5 function in interphase is difficult, however. Kinesin-5 is essential for proper progression through mitosis. Therefore a method has to be established, which depletes functional Kinesin-5 protein very fast at a desired time point. A promising assay could be the insertion of a specific 
surface view

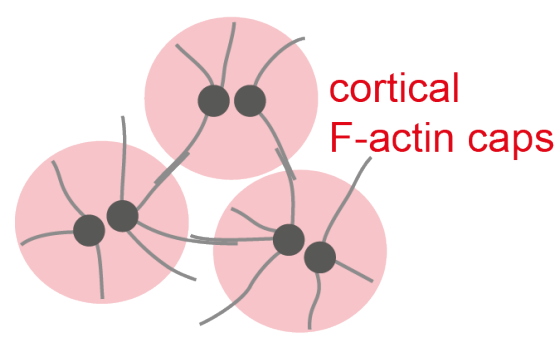

lateral view

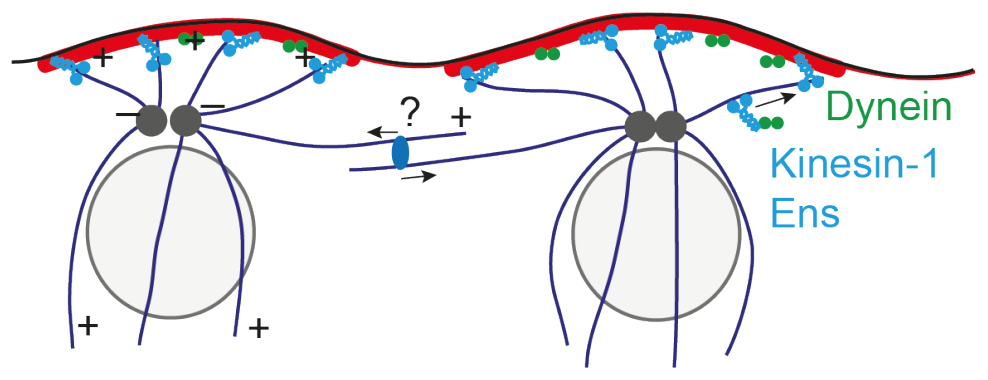

Figure 63: Model for the interaction of centrosomes with the cortex. Left site: surface view; right site: lateral view of the model. Microtubule asters (grey) emanate from centrosomes (grey) and overlap with each other. F-actin (red) forms a cap structure above the nuclei during interphase. Centrosomes are located between nuclei and actin cap. Kinesin-1 and its interactors Dynein and Ensonsin anchor microtubules to the actin cortex. An unknown protein, probably Kinesin-5 slides apart antiparallel microtubules at overlapping microtubule asters.

cleavage site, such as TEV cleavage site, between the head and tail compartments of the protein. Injection of highly concentrated and active TEV protease should recognise and destroy the targeted Kinesin-5 protein. However, currently TEV protease does not act fast enough, so it has to be injected early in syncytial development of Drosophila embryos, which interferes with subsequent mitoses. Once a suitable and fast method for depletion of functional Kinesin-5 at a specific time point is established, one could selectively address Kinesin-5 function in interphase without interfering with its mitotic contribution. 


\section{Bibliography}

[1] S. Acharya, P. Laupsien, C. Wenzl, S. Yan and J. Großhans. 'Function and dynamics of slam in furrow formation in early Drosophila embryo'. Developmental Biology 386.2 (2014), pp. 371-384.

[2] M. L. Basiri, S. Blachon, Y.-C. F. Chim and T. Avidor-Reiss. 'Imaging centrosomes in fly testes.' Journal of Visualized Experiments 79 (2013), e50938.

[3] B. Biersmith, Z. C. Liu, K. Bauman and E. R. Geisbrecht. 'The DOCK protein Sponge binds to ELMO and funcions in Drosophila embryonic CNS development.' PloS One 6 (2011), e16120.

[4] R. P. Brendza, K. B. Sheehan, F. R. Turner and W. M. Saxton. 'Clonal tests of conventional kinesin function during cell proliferation and differentiation.' Mol. Biol. Cell 11 (2000), pp. 1329-1343.

[5] R. P. Brendza, L. R. Serbus, W. M. Saxton and J. B. Duffy. 'Posterior Localization of Dynein and Dorsal-Ventral Axis Formation Depend on Kinesin in Drosophila Oocytes.' Current Biology 12 (2002), pp. 1541-1545.

[6] I. Brust-Mascher, P. Sommi, D. K. Cheerambathur and J. M. Scholey. 'Kinesin-5dependent poleward flux and spindle length control in Drosophila embryo mitosis.' Mol. Biol. Cell 20 (2009), pp. 1749-1762.

[7] A. Burakov, E. Nadezhdina, B. Slepchenko and V. Rodionov. 'Centrosome positioning in interphase cells.' Journal of Cell Biology 162 (2003), pp. 963-969.

[8] J. Cao, R. Albertson, B. Riggs, C. M. Field and W. Sullivan. 'Nuf, a Rab11 effector, maintains cytokinetic furrow integrity by promoting local actin polymerization.' Journal of Cell Biology 182 (2008), pp. 301-313.

[9] J. Cao, J. Crest, B. Fasulo and W. Sullivan. 'Cortical Actin Dynamics Facilitate Early-Stage Centrosome Separation.' Current Biology 20 (2010), pp. 770-776.

[10] U. del Castillo, M. Winding, W. Lu and V. I. Gelfand. 'Interplay between kinesin1 and cortical dynein during axonal outgrowth and microtubule organization in Drosophila neurons.' eLIFE 4 (2015), e10140.

[11] E. S. Chhabra and H. N. Higgs. 'The many faces of actin: matching assembly factors with cellular structures.' Nature Cell Biology 9 (2007), pp. 1110-1121. 
[12] E. N. Cytrynbaum, P. Sommi, I. Brust-Mascher, J. M. Scholey and A. Mogilner. 'Early spindle assembly in Drosophila embryos: role of a force balance involving cytoskeletal dynamics and nuclear mechanics.' Mol. Biol. Cell 16 (2005), pp. 4967-4981.

[13] V. E. Foe and B. M. Alberts. 'Studies of nuclear and cytoplasmic behaviour during the five mitotic cycles that precede gastrulation in Drosophila embryogenesis.' Journal of cell science 61 (1983), pp. 31-70.

[14] V. E. Foe, G. M. Odell and B. A. Edgar. 'Mitosis and morphogenesis in the Drosophila embryo: point and counterpoint.' In: The development of Drosophila melanogaster. Cold Spring Harbor, New York: Cold Spring Harbor Laboratory Press, 1993.

[15] S. L. Fullilove and A. G. Jacobson. 'Nuclear elongation and cytokinesis in Drosophila montana.' Developmental Biology 26 (1971), pp. 560-577.

[16] N. Hafi, M. Grunwald, L. S. van den Heuvel, T. Aspelmeier, J.-H. Chen, M. Zagrebelsky, O. M. Schütte, C. Steinem, M. Korte, A. Munk and P. J. Walla. 'Fluorescence nanoscopy by polarization modulation and polarization angle narrowing.' Nature Methods 11 (2014), pp. 579-584.

[17] M. M. Heck, A. Pereira, P. Pesavento, Y. Yannoni, A. C. Spradling and L. S. Goldstein. 'The kinesin-like protein KLP61F is essential for mitosis in Drosophila.' J. Cell Biol. 123 (1993), pp. 665-679.

[18] N. Hirokawa, N. Y., Y. Tanaka and S. Niwa. 'Kinesin superfamily motor proteins and intracellular transport.' Nat. Rev. Mol. Cell. Biol. 10 (2009), pp. 682-696.

[19] J. Januschke, L. Gervais, S. Dass, J. A. Kaltschmidt, H. Lopez-Schier, D. S. Johnston, A. H. Brand, S. Roth and A. Guichet. 'Polar Transport in the Drosophila Oocyte Requires Dynein and Kinesin I Cooperation.' Current Biology 12 (2002), pp. 1971-1981.

[20] A. L. Jolly, C.-H. Luan, B. E. Dusel, S. F. Dunne, M. Winding, V. J. Dixit, C. Robins, J. L. Saluk, D. J. Logan, A. E. Carpenter, M. Sharma, D. Dean, A. R. Cohen and V. I. Gelfand. 'A genome-wide RNAi screen for microtubule bundle formation and lysosome motility regulation in Drosophila S2 cells.' Cell Rep. 14 (2016), pp. 611-620.

[21] T. Kanesaki, C. M. Edwards, U. S. Schwarz and J. Grosshans. 'Dynamic ordering of nuclei in syncytial embryos: a quantitative analysis of the role of cytoskeletal networks.' Integr. Biol. 3 (2011), pp. 1112-1119.

[22] L.-R. Kao and T. Megraw. 'Centrocortin cooperates with centrosomin to organize Drosophila embryonic cleavage furrows.' Current Biology 19 (2009), pp. 937-942.

[23] J. R. Kardon and R. D. Vale. 'Regulators of the cytoplasmic dynein motor.' Nat. Rev. Mol. Cell. Biol. 10 (2009), pp. 854-865. 
[24] A. Kishino and T. Yanagida. 'Force measurements by micromanipulation of a single actin filament by glass needles.' Nature 334 (1988), pp. 74-76.

[25] T. Lecuit, R. Samanta and E. Wieschaus. 'slam encodes a developmental regulator of polarized membrane growth during cleavage of the Drosophila ambryo.' Current Biology 23 (2002), pp. 2110-2120.

[26] Z. Lv. 'Regulation of Actin dynamics by Formin in early Drosophila embryogenesis.' Dissertation (2014).

[27] A. Melkov, Y. Simchoni, Y. Alcyley and U. Abdu. 'Dynamic microtubule organization and mitochondrial transport are regulated by distinct Kinesin-1 pathways.' Biology open 4 (2015), pp. 1696-1706.

[28] T. Metzger, V. Gache, M. Xu, B. Cadot, E. S. Folker, B. E. Richardson, E. R. Gomes and M. K. Baylies. 'MAP and kinesin-dependent nuclear positioning is required for skeletal muscle function.' Nature 484 (2012), pp. 120-124.

[29] C. E. Monteith, M. E. Brunner, I. Djagaeva, A. M. Bielecki, J. M. Deutsch and W. M. Saxton. 'A mechanism for cytoplasmic streaming: Kinesin-driven alignement of microtubules and fast fluid flows.' Biophysical Journal 110 (2016), pp. 2053-2065.

[30] N. Peel, N. R. Stevens, R. Basto and J. W. Raff. 'Overexpressing centriolereplication proteins in vivo induces centriole overduplication and de novo formation.' Current Biology 17 (2007), pp. 834-843.

[31] A. D. Pilling, D. Horiuchi, C. M. Lively and W. M. Saxton. 'Kinesin-1 and Dynein are the primary motors for fast transport of mitochondria in Drosophila motor axons.' Mol. Biol. Cell 17 (2006), pp. 2057-2068.

[32] T. D. Pollard, L. Blanchoin and R. D. Mullins. 'Molecular mechanisms controlling actin filament dynamics in nonmuscle cells.' Annual Review of Biophysics and Biomolecular Structure 29 (2000), pp. 545-576.

[33] M. A. Postner, K. G. Miller and E. F. Wieschaus. 'Maternal effect mutations of the sponge locus affect actin cytoskeletal rearrangements in Drosophila melanogaster embryos.' Journal of Cell Biology 119 (1992), pp. 1205-1218.

[34] J. W. Raff and D. M. Glover. 'Centrosomes, and not nuclei, initiate pole cell formation in Drosophila embryos.' Cell 57 (1989), pp. 611-619.

[35] J. T. Robinson, E. J. Wojcik, M. A. Sanders, M. McGrail and T. S. Hays. 'Cytoplasmic dynein is required for the nuclear attachment and migration of centrosomes during mitosis in Drosophila.' J. Cell Biol. 146 (1999), pp. 597-608.

[36] E. D. Scheijter and E. Wieschaus. 'Functional elements of the cytoskeleton in the early Drosophila embryo.' Annu. Rev. Cell. Biol. 9 (1993), pp. 67-99. 
[37] J. Schmoranzer, J. P. Fawcett, M. Segura, S. Tan, R. B. Vallee, T. Pawson and G. G. Gundersen. 'Par3 and Dynein associate to regulate local microtubule dynamics and centrosome orientation during migration.' Current Biology 19 (2009), pp. 1065-1074.

[38] D. J. Sharp, K. R. Yu, J. C. Sisson, W. Sullivan and J. M. Scholey. 'Antagonistic microtubule-sliding motors position mitotic centrosomes in Drosophila early embryos'. Nat. Cell Biol. (1999), pp. 51-54.

[39] P. Sommi, D. Cheerambathur, I. Brust-Mascher and A. Mogilner. 'Actomyosindependent cortical dynamics contributes to the prophase force-balance in the early Drosophila embryo.' PLoS ONE 6 (2011), e18366.

[40] C. J. Staiger and L. Blanchoin. 'Actin dynamics: old friends with new stories.' Current Opinion in Plant Biology 9 (2006), pp. 554-562.

[41] J. Stein, H. Broihier, L. Moore and R. Lehmann. 'Slow as molasses is required for polarized membrane growth and germ cell migration in Drosophila.' Dev. Camb. Engl. 129 (2002), pp. 3925-3934.

[42] V. Stevenson, J. Kramer, J. Kuhn and W. E. Theurkauf. 'Centrosomes and the Scrambled protein coordinate microtubule-independent actin reorganisation.' Nature Cell Biology 3 (2001), pp. 68-75.

[43] V. Stevenson, A. Hudson, L. Cooley and W. E. Theurkauf. 'Arp2/3-dependent pseudocleavage furrow assembly in syncytial Drosophila embryos.' Current Biology 12 (2002), pp. 705-711.

[44] W. Sullivan, P. Fogarty and W. E. Theurkauf. 'Mutations affecting the cytoskeletal organization of syncytial Drosophila embryos.' Development 118.4 (1993), pp. $1245-1254$.

[45] H. H. Sung, I. A. Telley, P. Papadaki, A. Ephrussi, T. Surrey and P. Rørth. 'Drosophila Ensconsin promotes productive recruitment of Kinesin-1 to microtubules.' Developmental Cell 15 (2008), pp. 866-876.

[46] I. A. Telley, I. Gáspár, A. Ephrussi and T. Surrey. 'Aster migration determines the length scale of nuclear separation in the Drosophila syncytial embryo.' $P L o S$ ONE 6 (2011), e18366.

[47] J. A. Zallen, Y. Cohen, A. M. Hudson, L. Cooley, E. Wieschaus and E. D. Schejter. 'SCAR is a primary regulator of Arp2/3-dependent morphological events in Drosophila.' Journal of Cell Biology 156 (2002), pp. 689-701. 



\section{Acknowledgements}

Although the usual order of acknowledging would start with the supervisor, I first have to thank the "Großhans's lab Heat shock and Plate changing Service" from Dr. Zhiyi Lv and Deqing Kong. Without their help at the weekends, I would not have been able to generate even one germ line clone cross and this thesis would contain only very few data.

I am very thankful to Prof. Dr. Jörg Großhans for giving me the opportunity to carry out my $\mathrm{PhD}$ in his laboratory and for always being available for discussions and answering questions. Many thanks to him for all the support, advice, and guidance.

I would like to thank my thesis committee members Dr. Ronald Kühnlein and Dr. Arno Müller for their helpful suggestions and discussions during the thesis committee meetings.

I am thankful to all present and former members of the Großhans lab for the nice and warm-hearted working atmosphere, for their great discussions and their help with the methods. My thank goes to the members of the "Centrosome project": Dr. Takuma Kanesaki for introducing me in the project at the beginning of my $\mathrm{PhD}$ and $\mathrm{Dr}$. Maheshwar Reddy for sharing results and exciting discussions. I am very thankful to Dr. Michaela Clever for the supervision of Marvin Uhlig and her inspiring discussions always full of new ideas. Dr. Sreemukta Acharya, Roman Petrovsky, and Dr. Philip Laupsien, and Dr. Yujun Zhang helped me a lot especially in my starting time, but were also later a wonderful source of new perspectives to scientific questions. I would like to thank Dr. Hung-wei Sung for his helpful discussions and support especially with the RNAseq and Nanostring experiments. Stephanie Gröning I would like to thank for her kind introduction into the structural biology world and her help generating beautiful structure predictions. To Dr. Shuling Yan and Anja Schmidt my thank goes particularly for their introduction into the insitu hybridization method. I would like to thank my "Thursday meeting group" (Boyang Liu, Dr. Laszlo Henn, Saskia Spangenberg, and Marija Kojic): although our project topics differed a lot, we always found common features resulting in fascinating conversations. A special thank goes to Maria Kriebel for her charming sense of humour. I would also like to thank Kristina Hänecke and Johannes Sattmann as technicians providing the lab with all the stuff it needs.

I am thankful to the members of the "Department of Developmental Biochemistry" and the TAL lab for four years of great working environment (and the cakes of course).

I would like to thank Kai Yuan and Patrick O'Farrell for providing me the plasmids and flies for PCNA and HP1. My thank goes to Norbert Zielke and Bruce Edgar for providing the lab with the Fly-FUCCI lines. 
I would like Prof. Dr. Annette Zippelius, Dr. Ullrich Schwarz, Dr. Timo Aspelmeier, and Lutz Künneke for their collaboration in the centrosome project.

I thank Prof. Manfred Konrad and Dr. Nazim Nazimuddin for their collaboration in the establishment of a thymine assay for Drosophila embryos.

Finally I would like to thank my family and friends for their endless support and ecouragement.

Special thanks go to Stephan Anhalt for his patience and love. 


\section{Curriculum vitae}

\section{Personal data}

Name Franziska Winkler

Date of birth March 19, 1987

Place of birth Dresden, Germany

Address Petristeinweg 41, 99974 Mühlhausen / Thüringen, Germany

Email_ franziska_winkler@ymail.com

\section{Education}

From $07 / 2011 \quad$ PhD student at the Department of Developmental Biochemistry, University of Göttingen

PhD thesis title: "Role of Serine hydroxymethyltransferase in cell cycle control and early Drosophila development"

Advisor: Prof. Dr. Großhans; International PhD program "Genes and Development" (GGNB)

10/2010 - 04/2011 Graduant at the Department of Cytogenetics, Humboldt-University Berlin

Master thesis title: 'Interaction of the interband specific protein complex of Chriz and Z4 with Jil1, a kinase specific for the histone H3S10 of Drosophila melanogaster"

10/2008 - 05/2011 Studies of molecular biology, Humboldt-University Berlin Specialization: developmental biology, biochemistry, microbiology Graduation: Master of Science in Molecular Biology

04/2008 - 06/2008 Graduant at the Department of Plant Biochemistry, Freie Universität Berlin

Bachelor thesis title: " Establishment of an acitivity assay for Dihydropyrimidine dehydrogenase and characterization of Arabidopsis thaliana lines mutant for the pyrimidine recycling pathway"

10/2005 - 06/2008 Studies of biology, Freie Universität Berlin

Specialization: biochemistry, molecular biology, zoology, botanics

Graduation: Bachelor of Science in Biology

09/1997 - 06/2005 Grammar school (Johann-Andreas-Schubert-Gymnasium), Dresden 